\title{
THIN-SOURCE CONCENTRATION DEPENDENT DIFFUSION
}

VOLUME I

BY

This report was prepared as an account of work sponsored by the United States Govemment. Neither the United States nos the United States Department of Energy, nor any of their employees, nor any of their contractors, subcontractors, or their employees, makes any warranty, express or implied, or assumes any tegal liability or responsibility for the accuracy, completeness or usefulness of any information, apparatus, product or process disclosed, or represents that its use would infringe privately owned rights.

GENGHMUN ENG

B.S., The Cooper Union, 1972

Submitted in partial fulfillment of the requirements for the degree of Doctor of Philosophy in Physics in the Graduate College of the University of Illinois at Urbana-Champaign, 1978 


\section{DISCLAIMER}

This report was prepared as an account of work sponsored by an agency of the United States Government. Neither the United States Government nor any agency Thereof, nor any of their employees, makes any warranty, express or implied, or assumes any legal liability or responsibility for the accuracy, completeness, or usefulness of any information, apparatus, product, or process disclosed, or represents that its use would not infringe privately owned rights. Reference herein to any specific commercial product, process, or service by trade name, trademark, manufacturer, or otherwise does not necessarily constitute or imply its endorsement, recommendation, or favoring by the United States Government or any agency thereof. The views and opinions of authors expressed herein do not necessarily state or reflect those of the United States Government or any agency thereof. 


\section{DISCLAIMER}

Portions of this document may be illegible in electronic image products. Images are produced from the best available original document. 


\section{...this work is dedicated}

to my parents,

to my mentors;

Dr: David Lazarus and

Dr: Richard C. Extermann,

and to that elusive Muse called Wisdom. 


\section{THIN-SOURCE CONCENTRATION DEPENDENT DIFFUSION}

\section{Genghmun Eng, Ph.D. Department of Physics \\ University of Illinois at Urbana-Champaign}

The diffusion of $\left(\mathrm{Ca}^{++}\right)$in $\mathrm{NaCl}$ has been measured for various diffusion times and for the temperature range $\left(575^{\circ} \mathrm{C}-775^{\circ} \mathrm{C}\right)$, using a thinsource of ${ }^{45} \mathrm{Ca}$ tracer, rectangular geometry, and serial sectioning. The pre-diffusion surface concentration was approximately $3 \times 10^{16}$ (Ca)-atoms $/ \mathrm{cm}^{2}$, which, for an average penetration depth of $100 \mu \mathrm{m}-300 \mu \mathrm{m}$, produces a maximum (post-diffusion) impurity concentration comparable to or greater than the intrinsic cation vacancy concentration.

Such high impurity concentrations result in non-gaussian penetration profiles for the tracer, associated with a concentration dependent diffusivity, $D(C)$, governed by the non-linear diffusion equation:

$$
\frac{\partial C}{\partial t}=\frac{\partial}{\partial x} \cdot \dot{D}(C) \frac{\partial C}{\partial x} ; \quad \begin{aligned}
& D(C \rightarrow 0)=D_{0} \\
& C=\text { impurity concentration. }
\end{aligned}
$$

Whereas $D_{0}(T)$ is known from prior experiments performed at very low impurity concentrations of $\mathrm{Ca}^{++}$, little work has been done at higher concentrations because of the difficulty of solving the non-linear equation.

It is found that a solution is possible for a $D(C)$ process, using an experiment where two different samples with identical surface impurity concentrations are diffused for different times at the same temperature. A new data analysis procedure is developed which rigorously conserves total diffusant amount $\left(\int C(x, t) d x=N, N=\right.$ independent of time), and it allows 
an arbitrary function as an initial state; thus permitting the propagation from a measured initial state, $C\left(x, t_{R}\right)$, to a given final state, $C\left(x, t_{F}\right)$. If each $\left(\mathrm{Ca}^{+}\right)$-impurity introduces exactly one extra cation vacancy, maintaining local charge neutrality, one expects that:

$$
D(C)=D_{0}(1+\hat{\alpha} C)
$$

and if, in addition, vacancy equilibrium with extended defects is assumed, the $D(C)$-function is modified to:

$$
\mathrm{D}(\mathrm{C})=\mathrm{D}_{0}\left(\frac{\hat{\alpha}}{2} \mathrm{C}+\left[1+\left(\frac{\hat{\alpha}}{2} \mathrm{C}\right)^{2}\right]^{1 / 2}\right)=\mathrm{D}_{0}\left(1+\left[\frac{\hat{\alpha}}{2} \mathrm{C}\right]+.5\left[\frac{\hat{\alpha}}{2} \mathrm{C}\right]^{2}+\ldots\right) .
$$

The non-iinearity parameter, $\hat{\alpha}$, is proportional to the intrinsic cation vacancy concentration, and these 'polynomial' (in $C$ ) diffusivities are used to study the $\left(\mathrm{Ca}^{+}\right)$diffusion in NaCl process.

The analysis procedure provides an exact, but not closed, solution for the following diffusivity functions:

$$
\begin{aligned}
& D(C)=D_{0}(1+\alpha C) \\
& D(C)=D_{0}\left(1+\gamma C^{R}\right), \quad R=\text { positive integer } \\
& \therefore(C)=D_{0}\left(1+\alpha C+\beta C^{2}\right)
\end{aligned}
$$

with the concentration, $C(x, t)$, expressed as a series in the non-1inearity parameters $(\alpha, \beta, \gamma, \ldots), C(x, t)$ is found to order $-(\alpha, \beta)$, neglecting higher order terms.

For the present system, it is found that:

(1): $\mathrm{D}_{0}$, by itself, with no non-linear corrections, gives a poor datafit, except at the highest temperatures;

(2). $\quad D(C)=D_{0}(1+\alpha C)$ gives an improved datafit; with the amount of improvement diminishing at lower temperatures; 
(3). $D(C)=D_{0}\left(1+[\alpha C]+.5[\alpha C]^{2}\right)$ is a good datafit at high temperatures, but is barely better than $\mathrm{D}_{0}$ at lower temperatures;

(4). $D(C)=D_{0}\left(1+\alpha C+\beta C^{2}\right)$, with both $\alpha$ and $\beta$ adjustable, gives a good datafit over the entire temperature and time range, except for the shortest diffusion times;

(5). The $D_{0}(T)$ Arrhenius plot for the $D(C)=D_{0}\left(1+\alpha C+\beta C^{2}\right)$ function, after corrections for the non-linearity have been made, shows a distinct high temperature (H.T.) and lower-temperature (L.T.) region with:

$$
\begin{aligned}
& \mathrm{D}_{\mathrm{o}}(\mathrm{H} . \mathrm{T} .)=.642 \exp -[(1.69 \pm .02 \mathrm{eV}) / \mathrm{kT}] \mathrm{cm}^{2} / \mathrm{sec} \\
& \mathrm{D}_{\mathrm{o}}\left(\mathrm{L} . \mathrm{T}_{.}\right)=.431 \times 10^{-3} \exp -[(1.06 \pm .04 \mathrm{eV}) / \mathrm{kT}] \mathrm{cm}^{2} / \mathrm{sec} .
\end{aligned}
$$

The high-temperature function closely matches the $D_{0}(T)$ function obtained from low impurity concentration experiments. The lower-temperature function, combined with the sudden failure of the $D(C)=D_{0}\left(1+[C]+.5[C]^{2}\right)$ function at these lower temperatures, indicates the onset of a second diffusion process, one which would operate only at extremely high impurity concentrations. This low-temperature behavior is shown to be consistent with a breakdown of the conditions assumed for vacancy equilibrium.

$$
* * *
$$




\section{ACKNOWLEDGEMENTS}

First and foremost, I would like to thank my consistent advisor, Professor David Lazarus, for his infinite patience, guidance, faith, and trust, throughout the course of this long project. Too, another deep debt is owed to Dr. Joan L. Mitche11, who not only introduced me to the nuts and bolts of the Lazarus Laboratory, but who also introduced me to the intrigues of this particular subject. This work strongly rests on her pioneering work, and that of $\mathrm{Dr}$. Michael Feit; their work together, by the axiom of perversity, leading me strongly in the opposite direction.

A very special thanks is due to S. Dreifuss, for the proofs of existence and uniqueness, without which this universe would not quite be the same. Thanks also to my many friends and colleagues who made the rest of life as it is, and positively; most notably; the peoples Matusiewicz and $\mathrm{Mr}$. J. H. Anderson.

I must alșo thank Ms. Minnie Rindt for the excellent typing of a most difficult script; and of course; a grateful acknowledgement goes to the U. S. Department of Energy, under Contract EY-76-C-02-1198, for their continued financial support. 
TABLE OF CONTENTS

Page

PREFACE $\ldots \ldots \ldots \ldots \ldots \ldots \ldots \ldots \ldots \ldots \ldots \ldots \ldots \ldots \ldots \ldots \ldots \ldots \ldots \ldots \ldots \ldots$

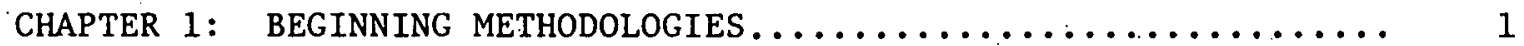

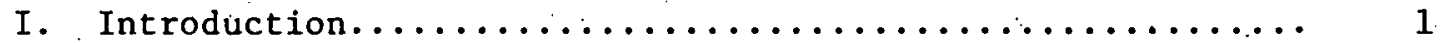

II. On Non-gaussian Penetration Profiles................. 3

III. Initial Conditions and the $t=\left(0, t_{R}\right)$ Time Range.......... 5

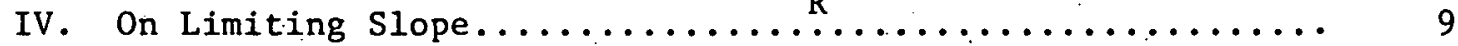

V. Beyond Limiting Slope......................... 14

CHAPTER 2: THE LINEAR DIFFUSION EQUATION................ 15

I. Orthogonal Functions and Propagators.............. 15

II. The Hermite Polynomial Solution................... 17

III. A Choice of Units.......................... 22

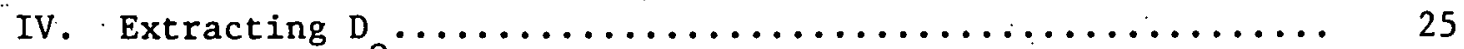

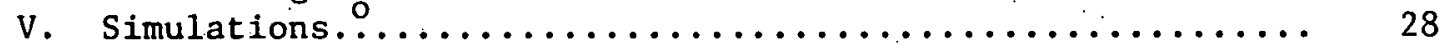

VI. Towards a Non-Linear Equation Analysis. ............. 31

CHAPTER 3: THE PHYSICAL ORIGINS OF A CONCENTRATION DEPENDENT

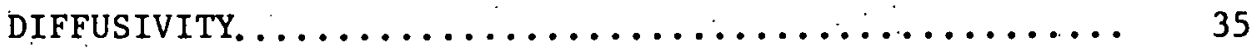

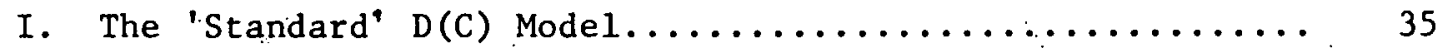

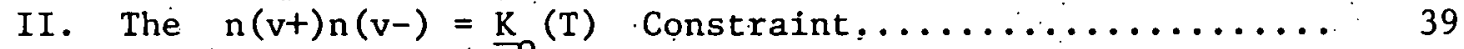

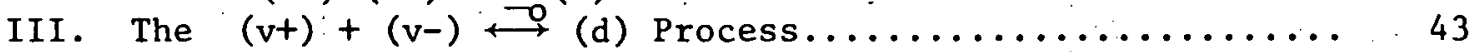

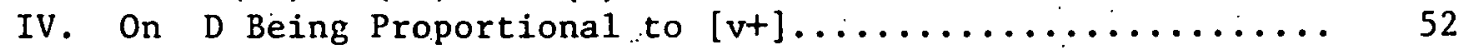

V. The Hopping Frequency...................... 56

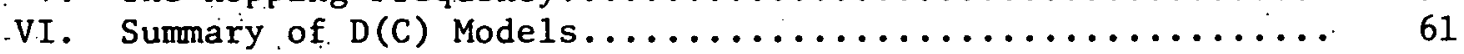

CHAPTER 4: NON-LINEAR ANALYSES AND THE $D=D_{0}(1+\alpha C)$ PROBLEM..... 66

I. The Lore of Concentration-Dependent Diffusion......... 66

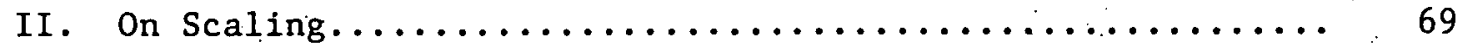

III. Notation and Sum Permutations................. 70

IV. The Dynamical Variables $(z, t)$ and the Parameter $D_{0} \ldots \ldots \ldots \quad 74$

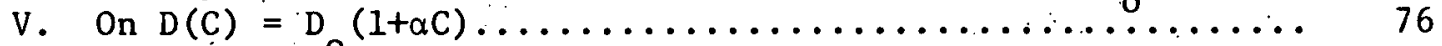

VI. Fundamental ${ }^{\circ}$ Properties of the $g(p, n)$-Equations......... 79

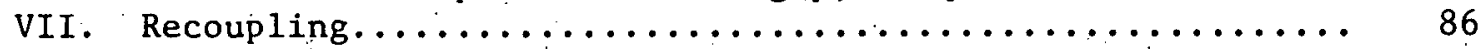

VIII. Review and Extensions........................ 88

CHAPTER 5: THE $D=D_{0}\left(1+\gamma C^{R}\right)$ AND $D=D_{0}\left(1+\alpha C+\beta C^{2}\right)$ PROBLEMS $\ldots \ldots .95$

I. General Formalism....................... 95 
CHAPTER 6: EXPLICIT CALCULATION OF THE $g(\mathrm{p}, \mathrm{n})$ FUNCTIONS....... 101

I. The Calculation Setup......................... 101

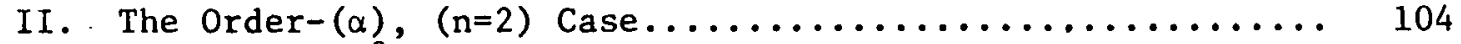

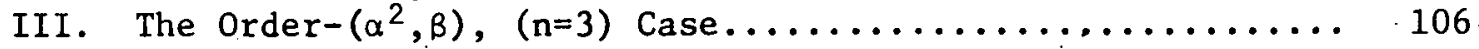

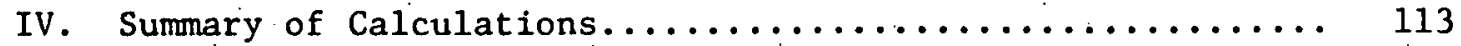

v. Non-linear and Multiple-time Correlations............. 116

CHAPTER 7: THE EXPERIMENTAL METHOD................... 123

I. Experimental Procedures..................... 123

II. Raw Data................................ 139

CHAPTER 8: RESULTS AND INTERPRETATIONS $\ldots \ldots \ldots \ldots \ldots \ldots \ldots \ldots \ldots \ldots$

I. Preliminaries............................ 168

II. The S-dataset, Average Temperature: $674.2^{\circ} \mathrm{C} \ldots \ldots \ldots \ldots \ldots \ldots \ldots$

III. The T-dataset, Average Temperature: $724.2^{\circ} \mathrm{C} \ldots \ldots \ldots \ldots \ldots .227$

IV. The U-dataset, Average Temperature: $774.9^{\circ} \mathrm{C} \ldots \ldots \ldots \ldots \ldots 243$

V. The R-dataset, Average Temperature: $624.7^{\circ} \mathrm{C} \ldots \ldots \ldots \ldots \ldots . \ldots 260$

VI. The Q-dataset, Average Temperature: $574.4^{\circ} \mathrm{C} \ldots \ldots \ldots \ldots \ldots . \ldots 269$

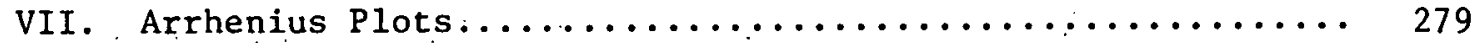

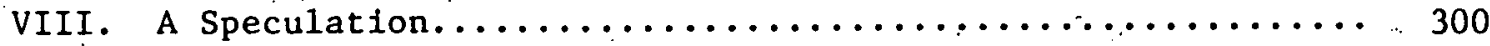

IX. Further Comments........................... 309

X. Conclusions...................................... 311

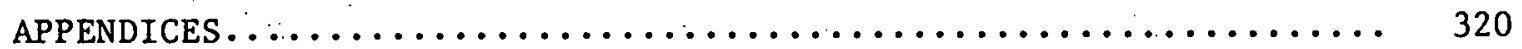

I. Additional Notes......................... 320

II. On the Convergence of Some Series of Hermite Polynomials.. 334

III. Creating a Continuous Datafit From Discrete Data........ 337

IV. Integrals of $\Gamma^{\nu}(\ell n \Gamma)^{\mathrm{m}} \ldots \ldots \ldots \ldots \ldots \ldots \ldots \ldots \ldots \ldots \ldots \ldots \ldots \ldots \ldots \ldots$

V. Coupling Coefficients for the $D=D_{0}(1+\alpha C)$

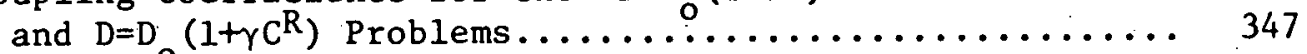

VI. Notes on $\left(\mathrm{Sc}^{++}\right) \rightarrow(\mathrm{NaCl})$ Diffusion............... 363

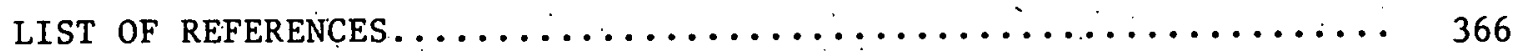

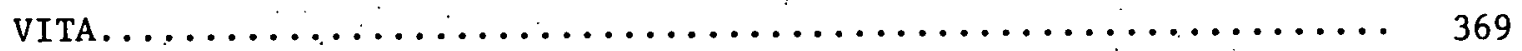




\section{PREFACE}

This thesis examines the general problems of "non-1inear" diffusion in a simple solid, from both experimental and theoretical viewpoints. The diffusion of $\left(\mathrm{Ca}^{++}\right)$in $\mathrm{NaCl}$ has been measured for various diffusion times and for the temperature range $\left(575^{\circ} \mathrm{C}-775^{\circ} \mathrm{C}\right)$, using a thin-source of ${ }^{45} \mathrm{Ca}$. tracer, rectangular geometry, and serial sectioning. The pre-diffusion surface concentration was approximately $3 \times 10^{16}$ (Ca)-atoms $/ \mathrm{cm}^{2}$, which, for an average penetration depth of $\sim 100 \mu-300 \mu$, produces a maximum (post-diffusion). impurity concentration comparable to or greater than the intrinsic cation vacancy concentration. Details of the experiment are given in Chapter 7.

Such high impurity concentrations result in non-gaussian penetration profiles for the tracer, associated with a concentration dependent diffusivity, $D(C)$, governed' by a non-linear diffusion equation:

$$
\frac{\partial C}{\partial t}=\frac{\partial}{\partial x} D(C) \frac{\partial C}{\partial x} ; \quad \begin{aligned}
& D(C \rightarrow 0) \equiv D \\
& C=\text { impurity concentration. }
\end{aligned}
$$

Whereas $D_{0}(T)$ is known from prior experiments performed at very. low impurity concentrations of $\mathrm{Ca}^{+}$, little work has been done at higher concentrations because of the difficulty of solving the non-linear equation.

It is found that a solution is possible for a $D(C)$ process by means of an experiment using at least two different samples with identical initial surface impurity concentrations, diffused for different times at the same temperature. A "two-time" experiment is necessary because the thin-source idealization, $C(x, t=0) \sim \delta(x)$, cannot be applied to a $D(C)$ process (Chapter 1 , Secs. II and III). 
If $C(x, t)$ is a gaussian, it plots as a straight line on [enc vs. $x^{2}$-axes, and non-gaussian profiles plot as "curved" functions, but with a tail (large $x^{2}$ ) region that is often close to a straight line, giving a "limiting-slope." The validity of this "limiting-slope". as a measure of $D_{o}$ is examined next, and it is found that the limiting slope can be inaccurate, even if $D=$ constant (Chapter 1 , Sec. IV, and Appendix I, Sec. 3). In Chapter 2, a method is discussed to analyze non-gaussian profiles for a two-time experiment, when $D=$ constant. This study of the constant diffusivity problem is motivated by the fact that an initial state, with a finite diffusion time, $t_{R}$, has a maximum concentration which is much less than the concentration of the initially deposited 'thin-source'. Thus, for times $t>t_{R}$, a $D=$ constant analysis for non-gaussian profiles might be sufficient to extract $D_{0}$ without resorting to a $D(C)$-analysis (Chapter 1 , Sec. III).

The method is based on orthogonal function expansions, which are briefly reviewed (in Chapter 2, Sec. I), and the solution to the linear diffusion equation, for non-gaussian profiles, is presented in terms of Hermite polynomials multiplied by a gaussian (Chapter 2 , Secs. II and IV). This solution emphasizes the fact that any non-gaussian initial state, with $\mathrm{D}=$ constant, must still propagate forward in a specified manner. $D_{0}$ is then extracted by comparing the propagated state to the measured final state (Chapter 2, Sec.IV). The way in which an initial state is parameterized in terms of the Hermite polynomials (times a gaussian) is also detailed (Chapter 2, Secs. III and V), using some additional results given in the appendices (Appendix II and III; also Appendix I, Secs. 1,2,4,9). Much of this orthogonal function methodology can be carried over to the non-linear D(C)-problem (Chapter 2, Sec. VI). 
In Chapter 3, the origin of a $D(C)$ process for $\left(\mathrm{Ca}^{++}\right)$diffusing in $\mathrm{NaCl}$ is reviewed (Chapter $3, \mathrm{Sec}$. I). If each $\mathrm{Ca}^{++}$impurity introduces exactly one extra cation vacancy, maintaining local charge neutrality, then:

$$
D(C)=D_{0}(1+\hat{\alpha} C)
$$

and if, in addition, vacancy equilibrium with extended defects is assumed, the $D(C)$-function is modified to:

$$
D(C)=D_{0}\left(\frac{\hat{\alpha}}{2} C+\left[1+\left(\frac{\hat{\alpha}}{2} C\right)^{2}\right]^{1 / 2}\right)=D_{0}\left(1+\left[\frac{\hat{\alpha}}{2} C\right]+.5\left[\frac{\hat{\alpha}}{2} \dot{C}\right]^{2}+\ldots\right) .
$$

This latter $D(C)$ function is the usual model that is applied to aliovalent Ion diffusion in strongly ionic solids, such as ( $\mathrm{Ca}^{++}$) diffusion in $\mathrm{NaCl}$. The non-1inearity parameter, $\hat{\alpha}$, is proportional to the inverse of the intrinsic vacancy concentration, and these $D(C)$ functions motivate the study (in Chapter 4 and 5) of a 'polynomial' (in C) diffusivity:

$$
D(C) \doteq D_{0}\left(1+\alpha C+B C^{2}+\gamma C^{3}+\ldots\right) .
$$

Both models rely on $\mathrm{D} \sim[\hat{\mathrm{v}}+]$, with $[\hat{\mathrm{v}}+]=$ total cation vacancy concentration, and if vacancy equilibrium with extended defects is assumed, it introduces the added condition of the familiar mass-action law:

$$
[\hat{\mathrm{v}}+][\hat{\mathrm{v}}-]=\underline{K}_{0}(\mathrm{~T})
$$

where $\underline{K}_{0}(T)$ is an equilibrium constant and $[\hat{v}-]=t o t a l$ anion vacancy concentration. These assumptions are carefully examined (in Chapter 3, Secs. II and IV), and it is found that a model with immobile or quasi-mobile anion vacancies, allowing for:

$$
(\mathrm{v}+)+(\mathrm{v}-) \longleftrightarrow(\mathrm{d}) ; \quad(\mathrm{d})=\text { divacancy }
$$

should equilibrate faster than a process involving equilibration with extended defects. This divacancy process (Chapter 3 , Sec. III) requires a new 
examination of the effective cation vacancy hopping frequency (Chapter 3 , Sec. V), which is a critical quantity determining a vacancy-controlled impurity diffusion coefficient.

These divacancy models still give a concentration dependent diffusivity, $D(C)$, but a small series expansion of the D.(C) function:

$$
D(C)=D_{0}\left(1+\alpha C+B C^{2}+\ldots\right)
$$

no longer requires $\beta=.5 \alpha^{2}$, as is true of the model using vacancy equilibrium with extended defects. The definitions of ' $D_{0}$ ' and ' $\alpha$ ' also differ among these various models, (Chapter 3, Sec. VI). In Chapter 4, the simplest non-linear diffusivity:

$$
\mathrm{D}(\mathrm{C})=\mathrm{D}_{\mathrm{O}}(1+\alpha \mathrm{C})
$$

is studied. The chapter begins with a survey of previous work on some $D(C)$ problems (Chapter 4 , Sec. I). The relationship between the non-1inearity parameter, $\alpha$, and the inverse of the intrinsic vacancy concentration is clarified (Chapter 4, Sec. II). The next section (Chapter 4, Sec. III) introduces notation used throughout the rest of the thesis; as well as 5 algebraic identities involving sum permutations that will be needed (primarily for use in Chapter 6 and Appendix V). A special transformation between $D_{0}$ and time, ' $t$ ', is given (Chapter 4, Sec. IV) which leads to a more aesthetic appearance of the non-linear. analysis, but its power of simplification is most evident in the calculation setup. for simultaneous analysis of many diffusion profiles at different diffusion times (Chapter 6 , Sec. V).

In Chapter 4 (Sec. V to VII), an exact, but not closed solution to the $D(C)=D_{0}(1+\alpha C)$ problem is outlined. The last section (Chapter.4, 
Sec. VIII) presents a summary of this method, as weli as the results of extensions of this method to handle the higher 'polynomial' (in C) diffusivities:

$$
\begin{aligned}
& D(C)=D_{0}\left(1+\gamma C^{R}\right), \quad R=\text { positive integer } \\
& D(C)=D_{0}\left(1+\alpha C+B C^{2}\right) .
\end{aligned}
$$

The method to solve these diffusivity functions can be made to exactly follow the procedure given for the $D(C)=D_{0}(1+\alpha C)$ problem, and this is explicitly demonstrated in Chapter 5. For each $D(C)$-polynomial, the propagated state, $C(x, t)$, is expressed as a series in the non-linearity parameters $(\alpha, \beta, \gamma, \ldots)$, and Chapter 6 presents a detailed determination of how a given initial state, $C\left(x, t_{R}\right)$ propagates forward in time, using the quadratic (in $C$ ) diffusivity. $C(x, t)$ is constructed to order $-\left(\alpha^{2}, \beta\right)$, neglecting terms of order $-\left(\alpha^{3}, \alpha \beta\right)$, which would have required the study of a cubic (in C) diffusivity. This chapter makes extensive use of the identities presented in Chapter 4 (Sec. IV) and of the results of Appendices IV and V. This procedure is then generalized (Chapter 6, Sec. V) to allow a simultaneous analysis of data for more than two diffusion times, using the same values of the parameters $\left(D_{0}, \alpha, \beta\right)$. It is this system that is used to analyze data:

Prior to this work, with the exception of work by Mitchell et al. ${ }^{+}$ and Feit et al., tr most of the analyses of thin-source concentration dependent diffusion had been restricted to using:

(1). limiting slope to determine $\mathrm{D}_{0}$, or

(2). finite-difference numerical calculations for non-gaussian profiles

${ }^{\dagger}$ J. L. Mitche11 and D. Lazarus, Phys. Rev. B 12, 734 (1975)

${ }^{\dagger}$ M. D. Feit, J. L. Mitche11, and D. Lazarus, Phys. Rev. B $\underline{8}, 1715$ (1973) 
even when $D=$ constant; or

(3). Low concentrations of impurities so that non-gaussian penetration profiles are avoided.

Even the approximate procedures of Mitchell and Feit began with a gaussian initial state, and they both used an iterative method which did not inherently preserve total diffusant amount. The present work, in contrast, allows an arbitrary function for an initial state, which has been shown to be necessary for a $D(C)$ process. The non-1inear propagation of a given initial state is explicitly constructed, preserving total diffusant amount. In addition, this work also provides a method to handle the more complicated problem of a polynomial (in $\mathrm{C}$ ) diffusivity.

In Chapter 7 , the experimental procedure used in this work is outlined, the fundamental experiment required being the measurement of at least two diffusion profiles at different diffusion times, using the same initial surface impurity concentration. The raw data points, $\left[\mathrm{C}_{i}, \mathrm{x}_{i}\right]$, are plotted at the end of Chapter 7 , along with a (polynomial) $\times$ (gaussian) fit to each dataset.: This fit provides an analytic representation of each dataset, which is then used in the analysis method developed here.

Chapter 8 (Secs. I-VI) presents the results of this non-linear diffusion analysis, using the quadratic (in C) diffusivity:

$$
D(C)=D_{0}\left(1+\alpha C+B C^{2}\right) \text {. }
$$

Major deviations from Arrhenius-type behavior are found, even after correcting for non-linearities using the quadratic diffusivity model, indicating probable failure of the mass-action law. The Arrhenius plot of $D_{0}(T)$ shows, for the high impurity concentrations used, a distinct high and low temperature 
region (Chapter 8, Sec. VII). Comparison with prior work indicates that true local equilibrium of vacancies may not, in fact, be established in the diffusion zone (Chapter 8 , Sec. VIII).

$$
\text { ** * }
$$


CHAPTER 1

\section{BEGINNING METHODOLOGIES}

\section{Introduction}

A good starting point in diffusion is Fick's Laws. ${ }^{1}$ First:

$$
\vec{J}=-\underline{D} \nabla C
$$

$\mathrm{J}$ is flux, $\mathrm{C}$ is concentration, and $\underline{D}$ is a phenomenological tensor quantity called the "diffusivity" or "diffusion coefficient.". Adding the condition of local conservation of mass

$$
\frac{\partial \dot{C}}{\partial t}=-\nabla \cdot \vec{J}
$$

gives:

$$
\frac{\partial C}{\partial t}=\nabla \cdot(\underline{D} \nabla C)
$$

In an isotropic medium, $\underline{D}=D \underline{I}$ where $\underline{I}$ is a unit tensor and $D$ is a scalar quantity. In 1-rectilinear dimension, if $\bar{D}=$ constant $\equiv \mathrm{D}_{0}$, the diffusion equation becomes the most basic parabolic equation:

$$
\frac{\partial C}{\partial t}=D_{0} \cdot\left(\frac{\partial}{\partial x}\right)^{2} C \equiv D_{0} \frac{\partial^{2} C}{\partial x^{2}}
$$

A boundary condition must be specified on Eq. (1.04) and using the $\delta$-function idealization of a "thin source":

$$
\underset{t \rightarrow 0}{\operatorname{Limit}} \mathrm{C}(\mathrm{x}, t)=\hat{1} \delta(x),
$$


it develops in time to the well-known gaussian: 2,3

$$
C(x, t)=\hat{i}\left(4 \pi D_{0} t\right)^{-1 / 2} \exp -\left(x^{2} / 4 D_{0} t\right)
$$

On a $\left[\ln C\right.$ vs. $\left.\dot{x}^{2}\right]$-graph, this gaussian solution is a straight line with slope $\left(-1 / 4 D_{0} t\right)$. Since ' $t$ ', the time of diffusion anneal, is known, $D_{0}$ is determined.

$$
* \star *
$$

Turning now to the specific problem of "thin source" diffusion in strongly ionic solids (as, for example, $\mathrm{Ca}^{++}$diffusion in $\mathrm{NaC} \ell$ ), it is we11-known that marked deviations from gaussian profiles occur in these systems $^{4}$ (even though strong ionicity of the host material is not a necessary prerequisite, as $\mathrm{Zn}^{++}$diffusion in GaAs also is strongly nongaussian ${ }^{5}$ ). These systems are believed to have a concentration dependent diffusion (c.d.d.) mechanism operating, giving rise to a $D(C)$, thereby invalidating Eq. (1.04). Many analyses deriving various $D(C)$-functions have been proposed, ${ }^{6}$ and these models are fairly easy to generate (see Chapter 3 for a more complete exposition).

The major difficulty encountered with the $D(C)$-functions is the fundamental non-1inearity of the diffusion equation (whose linearization returns one to: $\frac{\partial C}{\partial t}=D_{0}\left(\frac{\partial}{\partial x}\right)^{2} C$ ) and although many special cases for specific boundary conditions have been considered (see Chapter 4, sec. I), a consistent, calculable methodology relating directly to experiments and the physical conditions associated with them has not yet, to my knowledge, been developed. One of the aims in the succeeding sections and chapters is to develop such a methodology, and concurrently examine what constraints 
of experimental procedure it must be able to handle; and conversely what constraints, as a mathematical methodology, it imposes on the type of experiments that must be performed. Such a methodology to handle a $D(C)$ situation could be directly applicable to problems outside of aliovalent ion diffusion in strongly ionic crystals, although the ionic crystal systems are believed to have a large $D(C)$-effect and remain an ideal $D(C)-$ "test-system."

\section{On Non-gaussian Penetration Profiles}

If one begins with:

$$
\frac{\partial C}{\partial t}=D_{0}\left(\frac{\partial}{\partial \dot{x}}\right)^{2} C
$$

since it is a lst-order equation in ' $t$ ', following the classical mathematical physics procedure, one only needs to specify $C(x, t=0)$ from which $C(x, t)$ develops. A complete set of orthogonal functions appropriate to the given domain (here the domain is $t=(0, \infty), x=(-\infty,+\infty))^{7}$ is constructed and the boundary (initial) condition is expressed as a specific series of these functions, from which a final state is generated. Thus, the minimum procedure must be to measure an initial state, do the required orthogonal function expansion, propagate forward in time, and compare to a measured final state to extract the physical parameter $D_{0}$. The essential experiment is a.two-time (or multiple-time) correlation, and because both initial and final states are allowed to be non-gaussian, this means that a non-gaussian profile cannot prove that $D \neq c o n s t a n t$ :

On the other hand, with $D=$ constant, if at any time $C(x, t)$ is a gaussian, it must forever remain a gaussian. Then, and only then, is a 
1-time experiment valid (and, thus, practically every "normal" diffusion experiment is still correct). Thus, only if one has an initial state that is non-gaussian can it still proceed via $D=D_{0}$ : and still be (remaịn) non-gaussian.

Some physical situations with $D=D_{0}$ and with non-gaussian initial state are given next. Firstly, the idealization $c(x, t=0)=\hat{1} \delta(x)$ is not physically realizable because any laboratory-deposited "thinsource" is at least a few hundred Angstroms wide. However, the "thick" rectangular source $\left(C(x, t=0)=C_{A}\right.$ if $0<|x|<x_{A}$ and $C(x, t=0)=0$ if $|x|>x_{A}, D=$ constant $)$ has a well known time evolution in terms of complementary error functions: ${ }^{8}$

$$
C(x, t)=\left(\dot{C}_{A} / 2\right)\left(\operatorname{erf}\left[\left(x_{A}-x\right) /\left(4 D_{0} t\right)^{1 / 2}\right]+\operatorname{erf}\left[\left(x_{A}+x\right) /\left(4 D_{0} t\right)^{1 / 2}\right]\right)
$$

and thus, one can easily check to see if the non-gaussian behavior is due to having such a "thick-source." In practice, for a well-deposited "thin-source" ( $x_{A} \sim$ hundreds of angstroms), the complementary error functions and gaussian are virtually indistinguishable, and this cannot account for any large deviations from a gaussian in the initial state.

A physical situation that would have a strongly non-gaussian profile and $D=$ constant would be the diffusion of an ion-implanted profile, where the initial ion-injected state is determined by the mechanics of the implantation, but its time-development under diffusion anneal (high temperatures) would still be via the linear equation.

Another situation, closer to c.d.d., which also can create a nongaussian initial state is: If an experiment is performed measuring profiles at two times, $\left(t_{R}, t_{F} ; t_{F}>t_{R}\right)$ then the non-linearities in $D(C)$ can 
only be as important as $\zeta=\left[D\left(C_{A}\right) / D_{0}\right]-1$ where $C_{A}$ is the maximum concentration in the $C\left(x, t=t_{R}\right)$ profile. This is a lot less of a nonlinearity than using the "thin-source" near $t=0$ which has a maximal concentration (near the surface) far greater than $\mathrm{C}_{\mathbb{A}^{\cdot}}$. Thus, it is possible to have $t_{R}$ itself already sufficiently long after $t=0$ so that in the propagation from $t=t_{R}$ to $t=t_{F}, D(C) \simeq D_{0}$ may be a sufficient approximation. This would be a situation where one would have a non-gaussian initial $\left(t=t_{R}\right)$ state proceeding via $D=D_{0}$ where the only vestige of non-1inearity would be the initial $C\left(x, t_{R}\right)$ state being. distorted via strong non-linearities that had been operating from $0<t<t_{R}$. This is the "distorted initial state" approximation to the nonlinear equation, and an analysis via $\mathrm{D}=\mathrm{D}_{\mathrm{o}}$ for non-gaussian profiles should be one of the first "checks" before any more difficult analysis is attempted. It should demonstrate the need (or lack of it) for further methodology to be used where one suspects a $D(C)$ mechanism to be operating.

III.. Initial Conditions and the $t=\left(0, t_{R}\right)$ Time Range

Just as a "thin-source" was idealized as a $\delta$-function initial condition in solving the linear equation, a natural extension to $D(C)$ problems would be to use, again, the $\delta$-function as an initial condition. Having mentioned earlier that the non-linearity effect has a measure $\zeta=\left[D\left(C_{A}\right) / D_{0}\right]-1$, where $C_{A}$ is the maximum concentration in the $C\left(x, t_{R}\right)$ state, a $\delta$-function condition would give $\zeta \rightarrow \infty$. This hints at 
a difficulty elucidated even in the case of the simplest "non-linearity":

$$
D(C)=D_{0}(1+\alpha C)
$$

where $\alpha=$ non-1inearity parameter. Here,

$$
\frac{\partial C}{\partial t \cdot}=\frac{\partial}{\partial x} \cdot D(C) \frac{\partial C}{\partial x}=D_{0}\left(\frac{\partial}{\partial x}\right)^{2}\left(C+\frac{1}{2} \alpha C^{2}\right)
$$

Inserting $\mathrm{C} \rightarrow \hat{1} \delta(x)$, the right-hand side approaches

$$
D_{0}\left(\frac{\partial}{\partial x}\right)^{2}\left(\hat{1} \delta(x)+\frac{1}{2} \alpha(\hat{1} \delta(x))^{2}\right)
$$

and algebraic functions of $\delta$-functions (as $\delta^{2}(x)$ ), as well as algebraic functions of distributions in general, cannot even be (consistently) defined. 9 . The problem is impossible to solve.

Thus, from this start, the detailed shape of the initial state must be considered even in the "thin-source" approximation. A $\delta(x)$ cannot be used. Even then, near $t=0$, the actually deposited "thin-source" still has a very large concentration and can force

$$
\alpha C \gg 1
$$

Thus, extreme non-linearities in the diffusing system must occur for very short times. This almost guarantees that if a laboratory-thin source in the short time regime when $\mathrm{D}=$ constant develops into a gaussian, then even a small non-linearity in the diffusion equation ( $\operatorname{smal1} \alpha$ ) must, in the short time limit, develop into anything but a gaussian. This gives two important generalizations:

(1). If $D(C)$ is expanded as a power series

$$
D(C) \simeq D_{0}\left(1+\alpha C+\beta C^{2}+\gamma C^{3}+\ldots\right)
$$


any truncated series as an approximation to the actual $D(C)$ must fail as a good approximation when the concentration is large; i.e., this too must fail in the short time limit.

(2). Even if the full algebraic D(C) function was utilizable in calculations, in the large 'C' short time limit, the exact structure of $C(x, t \simeq 0)$ would be critical for the methematical analysis but, of course, it is practically impossible to measure with any accuracy experimentally.

This means one must stay bounded away from $t=0$ for an initial state. One must do a two-time (or multiple time) correlation experiment with finite, not-small times $\left(t_{R}, t_{F} \ldots\right)$. The $t_{R}$-state (shortest diffusion anneal in a multiple time correlation experiment) bears all the history of extreme non-1inearities encountered in $0<t<t_{R}$, and, so being an extremely distorted (from gaussian) initial state which cannot be a priori predicted, it necessarily follows that:

Any analysis in the chopped time interval $t=\left[t_{R}, \infty\right]$ (instead of $\left.t=\left[0^{+}, \infty\right]\right)$, while lowering the amount of non-linearity one needs to consider, conversely forces one to develop an analysis. method which must be able to handle an arbitrary shape penetration profile at $C\left(x, t_{R}\right)$ as an initial state.

One small advantage is gained from working in the chopped time interval $t=\left[t_{R}, \infty\right]:$ Since the main importance of this method of giving two samples identical histories between $t=\left(0, t_{R}\right)$ is that, in a c.d.d.process, the extreme non-linearities that must occur for very short times may be neglected, this method having ignored all details of the history before $t=t_{R}$ also allows the neglect of any corrections that might 
have been needed to explicitly account for the "finite warm-up time" when a diffusion anneal is performed at high temperatures. The exact behavior during this "warm-up time"-period (usually in the order of minutes for diffusion anneals that are in the order of hours) becomes just another $t<t_{R}$ process that is ignorable, as long as the time $t_{R}$ is at least as long as the minimum time which is needed for equilibration at the anneal temperature. A "warm-up time"-correction is now no longer needed!

The origin of the necessity for a "warm-up time"-correction (when the standard method is used of determining $D_{0}$ from the slope on a [ $\mathrm{nC}$ vs. $\left.x^{2}\right]$-graph of the penetrations profile) comes from the fact that a sample generally warms up approximately exponentially. A sample, originally at temperature $T_{1}$, when suddenly plunged into $a$. heat bath at $T_{2}$ heats up via:

$$
T(t)=T_{2}-\left(T_{2}-T_{1}\right) \exp -\left(t / t_{0}\right)
$$

where $t_{0}$ is a measure of the "warm-up time." Thus, it spends a significant amount of time being within, say, $5 \%$ of its final temperature, compared to the time it spends being within $5 \%$ of its initial temperature. And, because the diffusivity is usually a strongly increasing function of temperature, a: non-negligible amount of diffusion can take place before the true anneal temperature is reached.

We, having dispensed here with this "warm-up time"--correction, next consider a "cool-down time" or "quench-time" correction. Since the two samples which were "identical" in fabrication and identical in heating-up history, are diffused for different times, quite different profiles are quenched even though the quenching process is made identical. But not only because the "quench" is rapid (anneal temperatures $>500^{\circ} \mathrm{C}$, 
quench bath $=$ ice-water mixture: $0^{\circ} \mathrm{C}$ ), but also because the time spent within, say, $5 \%$ of its initial (now high) temperature is now extremely small (since the new equilibration temperature is $0^{\circ} \mathrm{C}$ ), the "cool-down time"-correction is entirely negligible.

\section{On Limiting Slope}

It is experimentally observed that often when curvature is seen on a [ lnC vs. $\left.x^{2}\right]$-graph, the deep penetration region appears to be a straight line, the slope of which, labelled "limiting slope," is often taken as a measure 4,10 of the diffusivity at vanishing concentration. Specifically, if $t_{R}$ is the experimental diffusion anneal time and L.S. = limiting slope, a limiting-slope-diffusivity, $\hat{D}_{0}$, can be constructed via:

$$
\text { L.S. } \equiv-1 / 4 \hat{D}_{0} t_{R} \text {. }
$$

For a c.d.d. process, a limiting-concentration-diffusivity can also be defined:

$$
\operatorname{Limit}_{C \rightarrow 0} D(C) \equiv D_{0}
$$

In the absence of any information on what $D_{0}$ "really" is, the ansatz of limiting slope is to set $\hat{D}_{0} \longleftrightarrow D_{0}$. The validity of this ansatz (or any cognate to it) can only be confirmed or refuted by comparison to a more rigorous analysis method.

For enhanced diffusion (diffusion via $D(C)$ where for all $C>0$, $\mathrm{D}(\mathrm{C})>\mathrm{D}_{\mathrm{O}}$, as would be expected for $\mathrm{Ca}^{++}$diffusion in NaCl), since the diffusion process is in some sense quicker, at a given time $t_{R}$, one might 
expect that the profile would be more "spread out" than it would have been had it proceeded for the entire time $\left(t=\left(0, t_{R}\right)\right)$ by $D=$ constant $=D_{0}$. Or, conversely, diffusion for a time $t_{R}$ with a $D(C)>D_{0}$ process, having a more spread out profile, could look similar to a diffusion via $D_{0}$ but for a longer time $t_{R}^{*}$. This is the "effective time" concept, and thus it would give:

$$
\text { L.S. } \equiv-1 / 4 \hat{D}_{0} \hat{t}_{R} \equiv-1 / 4 D_{0} t_{R}^{*} \cdot
$$

This takes an experimentally measured quantity. (L.S. on a [ $\operatorname{lnC}$ vs. $x^{2}$ ]graph), in conjunction with the experimentally measured time $\left(t_{R}\right)$, and allows one to create an "effective diffusivity": $\hat{D}_{0}$. Or, by using the (assumed known by another means) limiting-concentration-diffusivity $\left(D_{0}\right)$, one can alternately create an "effective time": $t_{R}^{\star}$. But if $D(C)>D_{0}$ this gives: $t_{R}^{*}>t_{R}$, therefore, $\hat{D}_{0}>D_{0}$. This indicates that the limiting slope ansatz is incorrect, and further, that the limiting-slope-diffusivity should be an upper bound to $\mathrm{D}_{0}$.

A similar argument can be constructed for "blocked" diffusion (diffusion via $D(C)$ where for all $C>0, D(C)<D$ as would be expected for $\mathrm{Na}^{+}$diffusion in $\mathrm{MgO}$ ) where $\mathrm{t}_{\mathrm{R}}^{*}<\mathrm{t}_{\mathrm{R}}$ and $\hat{\mathrm{D}}_{\mathrm{O}}$ is a lower bound to $\mathrm{D}_{0}$. Succinctly,

$$
\begin{gathered}
\operatorname{sign}\left[D(C)-D_{0}\right]=\operatorname{sign}\left[\hat{D}_{0}-D_{0}\right] \\
* * *
\end{gathered}
$$

There is a cognate to limiting slope for a two-time correlation. Given two profiles (for times $t_{R} ; t_{F}>t_{R}$ ), and two limiting slopes (L.S. ${ }_{1}$ and L.S. ${ }_{2}$ ):

$$
\text { L.S. } \left.{ }_{1}=-1 / \hat{x}_{R}^{2} \text { (short-time data, diffusion time } t_{R}\right)
$$




$$
\text { L.S. } \left.\dot{2}^{\prime}=-1 / \hat{x}_{F}^{2} \text { (long-time data, diffusion time } t_{F}\right) \text {, }
$$

another measure: a limiting-slope-diffusivity, two-time correlation, denoted $\hat{D}_{0}$ can be constructed via:

$$
\hat{x}_{F}^{2}=\hat{x}_{R}^{2}+4 \tilde{D}_{0}\left(t_{F}-t_{R}\right)
$$

A new limiting slope ansatz can be made -- that is to hope that $\tilde{D}_{0}$ is a good measured $D_{0}$. It can further be demonstrated that even if the profiles at $t_{R}$ and $t_{F}$ are non-gaussian, if the diffusion from $t_{R}$ to $t_{F}$ is via $D=D_{0}$, then indeed $\tilde{D}_{0} \equiv D_{0}$. And for this case, the earlier ascription:

$$
\text { L.S. }{ }_{1}=-1 / 4 \hat{D}_{0}^{1} t_{R}, \text { L.S. }{ }_{2}=-1 / 4 \hat{D}_{0}^{2} t_{F}
$$

would give different values for $\hat{D}_{0}^{1}$ and $\hat{D}_{0}^{2}$. Thus, in some sense, $\tilde{D}_{0}$ may be a better measure of $\mathrm{D}_{0}$ than $\hat{\mathrm{D}}_{0}$, most of the reason being that $\hat{\mathrm{D}}_{0}$ involves only the time difference $\left(t_{F}-t_{R}\right)$ and is independent of all the processes before $t_{R}$, whereas $\hat{D}_{0}$ is not. One drawback of $\tilde{D}_{0}$, though, is that $\operatorname{sign}\left[\tilde{D}_{0}-D_{0}\right]$ is no longer easily related to $\operatorname{sign}\left[D(C)-D_{0}\right]$. Also, just as $\hat{D}_{0}$ is not a good measure of $D_{0}$ for a $D(C)$ process, one may expect that if a strong $D(C)$ process is still operating in the time range $t=\left(t_{R}, t_{F}\right)$, that $\tilde{D}_{0}$ may not be a good measure of $D_{0}$ either.

$$
* * *
$$

With two measures of limiting-slope-diffusivity, $\tilde{\mathrm{D}}_{0}$ and $\hat{\mathrm{D}}_{0}$, in what sense they are related to $D_{0}$ given a $D(C)$ process; as well as what they are true measures of, is considered next. Given a multiple time correlation, with data having been taken for a set of times $\left(t_{1}, t_{2}, \ldots t_{N}\right)$, 
and defining $\left(\hat{\mathrm{x}}_{1}{ }^{2}, \hat{\mathrm{x}}_{2}{ }^{2}, \hat{\mathrm{x}}_{3}{ }^{2} \ldots \hat{\mathrm{x}}_{\mathrm{N}}{ }^{2}\right)$ from the 1imiting slopes of each profile:

$$
\text { L.S. }\left(t_{n}\right)=-1 / \hat{x}_{n}^{2}
$$

a set of $N$ values for $\hat{D}_{0}$ and $(N-1)$ values for $\tilde{D}_{0}$ can be constructed:

$$
\begin{aligned}
& \hat{\mathrm{D}}_{0}^{(n)}=\hat{x}_{n}^{2} / 4 t_{n} \quad(n=1,2, \ldots N) \\
& \tilde{D}_{0}^{(n)}=\left(x_{n}^{2}-x_{n-1}^{2}\right) /\left(4 t_{n}-4 t_{n-1}\right) \quad(n=2,3, \ldots N) .
\end{aligned}
$$

Expressing $\tilde{D}_{0}^{(n)}$ in terms of $\hat{D}_{0}^{(m)}$ by substituting for $\hat{x}_{m}^{2}$ gives alternately:

$$
\tilde{D}_{0}^{(n)}=\left[\hat{D}_{0}^{(n)} t_{n}-\hat{D}_{0}^{(n-1)} t_{n-1}\right] /\left(t_{n}-t_{n-1}\right)
$$

In the limit of a very large number of measurements. (so that $\left.t_{n}-t_{n-1}=\Delta t\right)$, the set of ordered pairs $\left(t_{n}, \hat{D}_{0}^{(n)}\right)$ approximate a function $\hat{D}_{\mathrm{O}}(t)$, and, furthermore, Eq. (1.23) becomes: ${ }^{11}$

$$
\tilde{D}_{0}(t)=\frac{\partial}{\partial t}\left(\hat{D}_{0}(t) \cdot t\right)
$$

The fact that limiting slope diffusivities are (thus shown to be) solely a function of time, may not be so surprising, for these diffusivities, $\tilde{\mathrm{D}}_{0}$ and $\hat{\mathrm{D}}_{\mathrm{O}}$, are already in the small concentration $(\mathrm{C} \rightarrow 0)$ as well as deep penetration: $(x \rightarrow \infty)$ limit, and the only finite parameter left is time.

$$
\text { Turning now to à c.d.d. equation, writing } D(C)=D_{0}(1+f(C))
$$

where

$$
\underset{C \rightarrow 0}{\text { Limit }} f(C)=0
$$

the following result is found: Whereas the fundamental c.d.d. equation is:

$$
\frac{\partial C}{\partial\left(D_{o} t\right)}=\frac{\partial}{\partial x}(1+f(C)) \frac{\partial C}{\partial x}
$$


the 'standard' method of limiting slope is replacing this non-linear equation with:

$$
\frac{\partial \mathrm{C}}{\partial\left(t \hat{D}_{0}(t)\right)}=\left(\frac{\partial}{\partial \mathrm{x}}\right)^{2} \mathrm{C}
$$

and the alternate method of limiting slope, involving $\tilde{\mathrm{D}}_{\mathrm{O}}$, replaces the non-linear equation with:

$$
\frac{\partial C}{\partial t}=\tilde{D}_{0}(t)\left(\frac{\partial}{\partial x}\right)^{2} C
$$

This assignment is used instead of reversing the roles of $\hat{D}_{0}$ and $\tilde{D}_{0}$ in Eqs. (1.26) and (1.27) because of the fundamental relationship:

$$
\tilde{D}_{0}(t) d t=d\left(\hat{D}_{0}(t) \cdot t\right)
$$

Just as $\hat{D}_{0}$ cannot be expected to be a good measure of $\mathrm{D}_{0}$, neither should $\tilde{D}_{\mathrm{o}}$ be.

Finally, if $\mathrm{D}(\mathrm{C})$ is monotonic in ' $\mathrm{C}$ ', since:

$$
\operatorname{sign}\left[\hat{D}_{0}(t)-D_{0}\right]=\operatorname{sign}\left[D(C)-D_{0}\right]
$$

it is not too unreasonable to assume $\hat{D}_{0}(t)$ is monotonic in ' $t$ ', deviating most from $D_{0}$ for short times, and least for long times. If this is so, then:

$$
\operatorname{sign}\left[\hat{\vec{D}}_{0}\left(t_{n-1}\right)-\hat{D}_{0}\left(t_{n}\right)\right]=\operatorname{sign}\left[D(C)-D_{0}\right],\left(t_{n}>t_{n-1}\right)
$$

and using Eq. (1.23) which can be re-written as:

$$
\tilde{D}_{0}\left(t_{n}\right)=\hat{D}_{0}\left(t_{n}\right)+t_{n-1}\left[\hat{D}_{0}\left(t_{n}\right)-\hat{D}_{0}\left(t_{n-1}\right)\right] /\left(t_{n}-t_{n-1}\right)
$$

the following relationship between the two "limiting-slope" diffusivities can be derived:

$$
\operatorname{sign}\left[\hat{D}_{0}\left(t_{n}\right)-\tilde{D}_{0}\left(\dot{t}_{n}\right)\right]=\operatorname{sign}\left[D(C)-D_{0}\right]
$$




\section{Beyond Limiting Slope}

The method of limiting slope is essentially an attempt to isolate

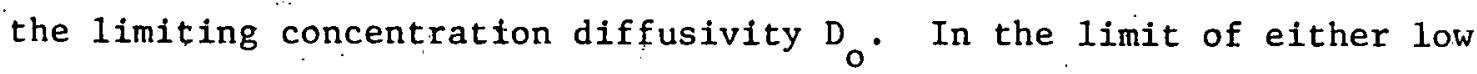
concentration or very long time $D(C) \simeq D_{0}$ must be a fair approximation, and the linear equation:

$$
\frac{\partial C}{\partial t}=D_{0}\left(\frac{\partial}{\partial x}\right)^{2} C
$$

must be marginally valid. Furthermore, the non-linearities that occur early in the diffusion anneal can distort the profile from gaussian, as well as alter the limiting slope $\hat{D}_{0}(t)$, and to a lesser extent alter $\tilde{D}_{0}(t)$. To stay away from extreme non-linearities, at least a two-time correlation must be performed (with times $t_{R}, t_{F} ; t_{F}>t_{R}$ ), and to isolate $\mathrm{D}_{0}$, one must be able to propagate via the linear equation using non-gaussian profiles.

In the next chapter, the method to isolate a "best" $D_{0}$ is outlined, assuming the linear equation to be valid. It will serve not only as a replacement for limiting slope, but also as a springboard from which a true $\dot{D}(\mathrm{C})$ analysis may be attempted. of course, whether the approximation $D(C) \simeq D_{0}$ is valid for the particular experiment at hand (with a given total aliovalent impurity and a given diffusion time) cannot be known a priori. 
CHAPTER 2

THE LINEAR DIFFUSION EQUATION

\section{Orthogonal Functions and Propagators}

Since $\frac{\partial C}{\partial t}=D_{0}\left(\frac{\partial}{\partial x}\right)^{2} C$ is a linear equation, a propagator exists

for it which will take any given initial state $C\left(x, t_{R}\right)$ into its cognate final state $C\left(x, t_{F}\right)$ for a given diffusivity $D_{0}$, via:

$$
C\left(x, t_{F} ; D_{0}\right)=: \int_{a 11} P\left(x, x^{\prime}, t_{F}, t_{R} ; D_{0}\right) C\left(x^{\prime}, t_{R}\right) d x^{\prime} .
$$

For the linear diffusion equation, the kernel specific to the $x=(-\infty, \infty)$ domain is: 12

$$
\begin{aligned}
& P\left(x, x^{\prime}, t_{F}, t_{R} ; \dot{D}_{0}\right)= \\
& \quad\left(4 D_{0}\left(t_{F}-t_{R}\right)\right)^{-1 / 2} \exp -\left[\left(x-x^{\prime}\right)^{2} /\left(4 D_{0}\left(t_{F}-t_{R}\right)\right)\right] .
\end{aligned}
$$

Although this allows for any $C\left(x, t_{R}\right)<A \exp +\left(x / x_{A}\right)^{2}$ (i.e., $C\left(x, t_{R}\right)$ must be of the form such that there exists some $\left(A, x_{A}\right)$ which makes the inequality true), it neither specifies the orthogonal function set for this domain, nor does it in any easy way allow handling of the true experimental problem: Given a particular $C\left(x, t_{R}\right)$ and $C\left(x, t_{F}\right)$, find the best $D_{0}$ that will take the given initial state into the given final state. Even if $C\left(x, t_{R}\right)$ were continuous data instead of experimental points $\left[x_{i}, C\left(x_{1}, t_{R}\right)\right]$, unless it was some a priori known specific analytical function (as $\left.C\left(x, t_{R}\right)=A \exp -\left(x / x_{A}\right)\right)$, one is reduced to either 
performing numerical integrals or expanding $C\left(x, t_{R}\right)$ as a series in some pre-selected functions $q_{n}(x)$, i.e.:

$$
C\left(x, t_{R}\right)=\sum_{n=0}^{m} v_{n} q_{n}(x)
$$

Given that the profile-specific parameters, $V_{n}$, could be found, only the set of $(m+1)$ integrals:

$$
\begin{gathered}
Q_{n}\left(x, x_{0}\right)=\int_{a 11 x^{\prime}}\left(1 / x_{0}\right) \exp -\left[\left(x-x^{\prime}\right)^{2} / x_{0}^{2}\right] q_{n}\left(x^{\prime}\right) d x^{\prime} \\
x_{0}=\left(4 D_{0}\left(t_{F}-t_{R}\right)\right)^{+1 / 2}
\end{gathered}
$$

would need to be calculated. But if one must expand $C\left(x, t_{R}\right)$ as a function series in terms of some pre-selected functions, then, if the $q_{n}(x)$ were chosen to be members of any complete orthogonal function set, the following advantages would be gained: ${ }^{13}$

(1). Whereas the best $m$-function for $\left(q_{0}, q_{1}, q_{m-1}\right)$. gives coefficients $\left(v_{0}, v_{1}, \ldots v_{m-1}\right)$, the best $(m+1)$-function fit using $\left(q_{0}, q_{1}, q_{m}\right)$ in general involves a whole new set of coefficients $\left(v_{0}^{\prime}, v_{1}^{\prime}, v_{2}{ }^{\prime} \ldots v_{m}^{\prime}\right)$, but if the $\left\{q_{m}\right\}$-set are orthogonal functions, the best $(m+1)$-orthogonal-function fit retains the first m-coefficients $\left(v_{0}, v_{1} \ldots v_{m-1}\right)$ and requires the calculation of one new coefficient: $V_{m}$.

(2). An m-orthogonal-function $f i t$ is the best m-function fit possible.

(3). The mean-squared deviation between the approximating series and the original function is a minimum; i.e.,

$$
\int\left[C\left(x, t_{R}\right)-\sum_{n=0}^{m} v_{n} q_{n}(x)\right]^{2} d x=\text { minimum. }
$$


Finally, if the expansion is done in terms of orthogonal functions, the set of orthogonal functions which solves the diffusion equation might as well be chosen, since the propagation of the set which solves the (1inear), equation is simplest.

As recently as 1975 , M.J. Turunen ${ }^{14}$ published an article detailing a finite difference methodology for analyzing non-gaussian profiles using the linear equation. His method requires knowledge of $c(x, t-\Delta t)$, $C(x, t), C(x, t+\Delta t)$ and is in essence, a triple-time correlation. No mention was made of the existence of an equivalent analytical formulation, nor of this "proper" orthogonal function set which solves the linear equation. This solution, known to the classical mathematicians, ${ }^{15}$ is reviewed next.

\section{The Hermite Polynomial Solution}

The fundamental 'thin-source' solution

$$
\ddot{C}_{0}(x, t)=\left(4 D_{0} t\right)^{-1 / 2} \exp -\left(x^{2} / 4 D_{0} t\right)
$$

solves the equation:

$$
\frac{\partial C}{\partial t}=D_{0}\left(\frac{\partial}{\partial x}\right)^{2} C
$$

But this equation has the additional "symmetry" that given any $\hat{c}(x, t)$ which solves the equation, every one of the following:

$$
\left(\frac{\partial}{\partial x}\right) \hat{c},\left(\frac{\partial}{\partial x}\right)^{2} \hat{c},\left(\frac{\partial}{\partial x}\right)^{3} \hat{c}, \ldots\left(\frac{\partial}{\partial x}\right)^{m} \hat{c},\left(\frac{\partial}{\partial t}\right) \cdot \hat{c},\left(\frac{\partial}{\partial t}\right)^{2} \hat{c}, \ldots
$$

will also solve the equation. In general, these may not be 'new' or 'linearly independent' solutions, but because the 'thin-source' function 
$c_{0}(x, t)$ is not separable (i.e., $C_{0}(x, t) \neq C_{1}(x) C_{2}(t)$ ), one is guaranteed that:

$$
c_{m}(x, t) \equiv\left(\frac{\partial}{\partial x}\right)^{m} c_{0}(x, t)
$$

must be a new solution. Explicitly:

$$
C_{m}(x, t)=\left(4 D_{0} t\right)^{-1 / 2}\left(\frac{\partial}{\partial x}\right)^{m} \exp -\left(x^{2} / 4 D_{0} t\right)
$$

Since ' $t$ ' is treated as a constant with respect to $\left(\frac{\partial}{\partial x}\right)$-operations,

$$
C_{m}(x, t)=\left(4 D_{0} t\right)^{-1 / 2} a^{m}\left(\frac{\partial}{\partial(a x)}\right)^{m} \exp -\left(x^{2} / 4 D_{o} t\right)
$$

and one is free to choose $a=\left(4 D_{0} t\right)^{-1 / 2}$. Defining $z^{2}=x^{2} / 4 D_{0} t$, one then has:

$$
C_{m}(x, t)=\left(4 D_{0} t\right)^{-(m+1) / 2}\left(\frac{\partial}{\partial z}\right)^{m} \exp -z^{2}
$$

Since the fundamental solution $C_{0}(x, t)$ is a function which has no axis crossings, the function:

$$
q_{m}(z)=\left(\frac{\partial}{\partial z}\right)^{m} \cdot \exp -z^{2}
$$

as a function of ' $z$ ', must cross the axis exactly ' $m$ '-times. Thus, each $C_{m}(x, t)$ is linearly independent (though not necessarily orthogonal); and the set $\left\{q_{m}\right\}$ is constructable and ortho-normalizable. Furthermore, because the fundamental solution $C_{0}(x, t)$ is of the class of (exp-[polynomial]), this gives the additional simplification that $q_{m}=$ (polynomiai) $x$ $\exp -\left(z^{2}\right)$. In this specific case, the polynomial is well-known: 16

$$
\left(\frac{d}{d z}\right)^{m} \exp -z^{2}=(-1)^{m} H_{m}(z) \exp -z^{2}
$$

where $H_{m}(z)$ is the $m^{\text {th }}$ Hermite Polynomial, and as a system of classical polynomials, they are already complete and orthogonal. Thus, each $\mathrm{C}_{\mathrm{m}}$ by itself is orthogonal to any $\left(C_{m}\right)$ and one has the Generalized-Thin-Source 
solution to the linear equation as:

$$
C(x, t)=\sum_{m=0}^{\infty} \hat{A}_{m}\left(4 D_{0} t\right)^{-(m+1) / 2} H_{m}(z) \exp -z^{2} ; z^{2}=x^{2} / 4 D_{o} t
$$

where the $\hat{A}_{m}^{\prime}$ 's are arbitrary constants; some additional properties of Hermite Polynomials are: 17

$$
\begin{aligned}
& H_{m}(z) \text { has parity }(-1)^{m}, H_{o}(z)=1 \\
& \frac{\mathrm{d}}{\mathrm{dz}} \cdot \mathrm{H}_{\mathrm{m}}(\mathrm{z})=2 \mathrm{mH} \mathrm{H}_{\mathrm{m}-1}(\mathrm{z}) \\
& H_{m+1}(z)=2 z H_{m}(z)-2 m H_{m-1}(z) \\
& \frac{d}{d z}\left[H_{m}(z) \exp -z^{2}\right]=-H_{m+1}(z) \exp -z^{2} \\
& \mathrm{H}_{2 \mathrm{~m}}(0)=(-1)^{\mathrm{m}} 2^{\mathrm{m}}(2 \mathrm{~m}-1) ! !=(-1)^{\mathrm{m}}(2 \mathrm{~m}) ! /(\mathrm{m} !) \\
& \int H_{m}(z) H_{n}(z) \exp -z^{2} d z=\delta(m=n) \pi^{1 / 2} n ! 2^{n}
\end{aligned}
$$

The diffusion geometry for 'thin-source' diffusion is usually not the interval $x=(-\infty ; \infty)$ which is the domain of the Hermite polynomial solution just derived, but rather the interval: $x=(0, \infty)$. Using reflection symmetry, the $x=(-\infty ; 0)$ interval can be mirrored as being $(+)$ or $(-)$ the function on the $x=(0, \infty)$ interval. In the $(+)$-case, only. the m=even part of the solution is needed, and in the $(-)$-case, only the m=odd part is. We choose here, and for the rest of this thesis, the $(+)$ convention, giving the full solution applicable to the experimentally used geometry as:

$$
C(x, t)=\sum_{p=0,1,2}^{\infty} \hat{A}_{2 p}\left(4 D_{0} t\right)^{-p-1 / 2} H_{2 p}(z) \exp -z^{2}
$$

As a further choice, throughout the course of this work, the double 
infinite interval $\mathrm{x}=(-\infty, \infty)$ will be used along with an even-parityfunctions-only restriction, and unless otherwise stated, all integrals on ' $x$ ' will have implicit limits $x=(-\infty, \infty)$. All the analyses using $\mathrm{H}_{2 \mathrm{p}}(\mathrm{z})$ are straightforwardly applicable to the $\mathrm{H}_{\mathrm{m}}(z), \mathrm{m}=$ odd-integer case, even though that will not explicitly be considered.

\section{* * *}

Knowing that the fundamentally pure gaussian solution $C_{0}(x, t)$ converges to $\delta(x)$ as $t \rightarrow 0$, what these additional solutions $C_{m}(x, t)$ converge to as $t \rightarrow 0$ can easily be found. Since these additional. solutions were constructed from $\left(\frac{\partial}{\partial x}\right)^{m} C_{0}(x, t), C_{m}(x, t)$ must give:

$$
\operatorname{Limit}_{t \rightarrow 0} C(x, t)=\sum_{m=0}^{\infty} \hat{A}_{m} \delta^{(m)}(x),
$$

where the superscript. (m) denotes the $\mathrm{m}^{\text {th }}$ derivitive. This $\delta$-function series is the most general distribution compact on the interval containing $x=0.18$ The physical significance of these ${ }_{\delta}^{(m)}(x)$ terms is clear: One does not expect the need for terms involving other than $\delta(x)$ in describing a physically constructed laboratory-like "thin-source" (where $\delta(x)$ is already a too-thin idealization of a source hundreds of angstroms thick). However, because, the first measurement is at a quite finite time, $t_{R}$, with the second measurement at $t_{F}$, given that the diffusion from $t=t_{R}$ to $t=t_{F}$ obeys the linear equation, some process before $t=t_{R}$ can distort the initial profile from gaussian. It is this 'distortion' process that makes the additional functions $H_{m}(z)$ exp-z necessary in the description of the $t=t_{R}$ state, which would then be equivalent to the introduction of $\delta^{(m)}(x)$ 
terms into the $C(x, t=0)$ series. One reminder, though, is that the physical situation is really bounded away from $t=0$, and the vanishing time limit is outside the physical domain which is the open interval $t=\left(t_{R}, \infty\right)$

One also sees that a non-gaussian profile $\hat{(A}_{\mathrm{m}} \neq 0$. for some $m \neq 0)$ remains non-gaussian, and that the higher order $(m \neq 0)$ terms decrease in time faster and faster (so that the main contribution as $t \rightarrow \infty$ is the pure gaussian). Finally, since at any given time, the full solution $C(x, t)$ is representable as (polynomial in $z$ ) $\times$ exp-z $z^{2}$, we show even in the simplest case of $C(x, t)=\left(1+\varepsilon z^{2}\right)$ exp-z ${ }^{2}$ that even though there is a limiting slope (on a [ lnC vs: $z^{2}$ ]-graph), the limiting intercept diverges! It is as follows:

$$
\ln C=-z^{2}+\ln \left(1+\varepsilon z^{2}\right)
$$

writing $\ell n C$ as $f(y)$ where $y=z^{2}$; then a graph of. [f(y) vs. $y$ ] gives

$$
f(y)=-y+\ln (1+\varepsilon y) .
$$

For very large $y_{0}$, approximating $f(y)$ near $y=y_{0}$ by a straight line gives:

$$
\begin{aligned}
f(y) & \simeq A+B\left(y-y_{0}\right) ; A=\left[-y_{0}+\ln \left(1+\varepsilon y_{0}\right)\right], \\
B & =\left[-1+\varepsilon /\left(1+\varepsilon y_{0}\right)\right]
\end{aligned}
$$

The "limiting splope" is $B$, which as $y_{0} \rightarrow \infty$ approaches $(-1)$. The limiting intercept (straight line fit extrapolated to $y=0$ ) is (A-By. ${ }_{0}$ ). It is easily seen that:

$$
A-B y_{0} \simeq\left(\ln \left(\varepsilon y_{0}\right)-1\right)+\operatorname{order}-\left(\left[\varepsilon y_{0}\right]^{-1}\right)
$$

which diverges for large $y_{0}$. 


\section{A Choice of Units}

In this section and for the rest of this thesis, unless otherwise stated, the units we will work in are the following:

$$
\begin{aligned}
& 1 \mathrm{xu}=1 \text { unit of distance }=10 \mu=10 \mathrm{microns} \\
& 1 \mathrm{tu}=1 \text { unit of time }=10^{4} \mathrm{sec} \\
& 1 \mathrm{du}=1 \text { unit of diffusivity }=10^{-10} \mathrm{~cm}^{2} / \mathrm{sec} \\
& 1 \mathrm{du}=1 \mathrm{xu}^{2} / 1 \mathrm{tu} \\
& 1 \mathrm{cu}=1 \text { unit of concentration }=1 \mathrm{Amt} \cdot 11 \mathrm{xu} \\
& 1 \text { Amt }=1 \text { Amount }=10 \text { nanomoles } / \mathrm{cm}^{2}
\end{aligned}
$$

A note on the concentration units is in order. Since the diffusion geometry consists of one relevant rectilinear dimension, with:

$$
\int_{a 11} C(x, t) d x=N
$$

where $N=$ Total Amount of tracer atoms in sample, and since for a "thinsource" geometry, ' $\mathrm{N}$ ' is fixed, it could take on the units of 'Numbers' (i.e., no units at all). Yet the physically relevant microscopic quantity is not $\mathrm{C}$ in Amt:/xu nor $\mathrm{N}$ in Amounts but rather ppm. impurity; for if the $1 \mathrm{Amt} / \mathrm{xu}$ impurity is physically spread on a planar surface (perpendicular to the $x$ direction) of $1 \mathrm{~cm}^{2}$, the inherent concentration is 10 times more than if it were spread on a face of $10 \mathrm{~cm}^{2}$. . Thus, the true unit of concentration is taken as:

$$
1 \mathrm{cu}=1 \text { nanomole } / \mathrm{cm}^{2} \text { micron }
$$

In these units, the concentration of host cation lattice sites in $\mathrm{NaCl}$ is: 
Since the primary experimental system of interest here is $\mathrm{Ca}^{++} \rightarrow \mathrm{NaCl}$, one can easily convert from concentration units to ppm. impurity by dividing by $3.705 \times 10^{-3}$ giving

1 Amt. $/ x u \leftrightarrow 269.91$ ppm. (in NaCl)

$* * *$

Having chosen all our units, the entire profile $C(x, t)$ is now specified. Suppose now two profiles were measured: $\tilde{C}_{R}\left(x, t_{R}\right), \tilde{C}_{F}\left(x, t_{F}\right)$, with:

$$
\int_{-\infty}^{\infty} \tilde{C}_{R}\left(x, t_{R}\right) d x=N(R), \int_{-\infty}^{\infty} \tilde{C}_{F}\left(x, t_{F}\right) d x=N(F)
$$

since the linear equation scales (i.e., if $C(x, t)$ șolves the equation, so does $\lambda C(x, t)$ for any $\lambda)$ it does not matter if $N_{F} \neq N_{R}$. Defining a new $C_{R}\left(x, t_{R}\right), C_{F}\left(x, t_{F}\right)$ so that:

$$
\int_{-\infty}^{\infty} C_{R}\left(x, t_{R}\right) d x \doteq \hat{1} \quad, \int_{-\infty}^{\infty} C_{F}\left(x, t_{F}\right) d x=\hat{1}
$$

this gives two normed profiles, each of which must next be parameterized as a series in Hermite polynomials. Since exponents are pure numbers, we introduce the "characteristic lengths" $x_{R}$ (for $C\left(x, t_{R}\right)$ ) and $x_{F}$ (for $C\left(x, t_{F}\right)$ ) giving:

$$
\begin{aligned}
C_{R}\left(x, t_{R}\right) & =\sum_{p=0}^{N I} B_{p} H_{2 p}\left(x / x_{R}\right) \exp -\left(x / x_{R}\right)^{2} \\
C_{F}\left(x, t_{F}\right) & =\sum_{p=0}^{N F} \tilde{B}_{p} H_{2 p}\left(x / x_{F}\right) \exp -\left(x / x_{F}\right)^{2}
\end{aligned}
$$


of course, the values of the expansion coefficients $\left\{B_{p}\right\}$ and $\left\{\tilde{B}_{p}\right\}$ depend on the value of $x_{R}$ and $x_{F}$, respectively, but a procedure to choose $x_{R}$ and $x_{F}$ must be specified. In some sense $x_{R}^{2}$ is analogous to $4 D_{0} t_{R}$ and $x_{F}{ }^{2}$ is analogous to $4 D_{0} t_{F}$; but even though $t_{R}$ and $t_{F}$ are known, since $D_{0}$ is that which is to be determined, to make the explicit assignment $x_{R}^{2} \sim 4 D_{0} t_{R}$ and $x_{F}^{2} \sim 4 D_{0} t_{F}$ would be equivalent to deciding a priori on an answer for $\mathrm{D}_{0}$.

The more correct viewpoint is to note that a series of orthogonal functions is capable of matching an arbitrary initial state. To specify $x_{R}$ is just to complete the specification of the orthogonal functions being used. With (any) $x_{R}$ chosen, the orthogonal function series should still be able to match any $c\left(x, t_{R}\right)$ state. Thus, $x_{R}$ (and consequently $x_{F}$ ) are completely arbitrary.

There is one additional proviso, which concerns the convergence of the Hermite polynomial series (i.e., does the series allegedly representing $\dot{C}\left(x, t_{R}\right)$ (or $C\left(x, t_{F}\right)$ ) really converge for any value of $x_{R}$ or $x_{F}$ chosen?). We state the following result (with details for interested readers in Appendix II):

If a state $C\left(x, t_{R}\right)$ has a tail which decreases as fast as or faster than $A \exp -\left(x / x_{A}\right)^{2}$ for some $A, x_{A}$, then the series

$$
C\left(x, t_{R}\right) \simeq \sum_{m=0}^{N I} B_{m} H_{2 m}\left(\frac{x}{x_{R}}\right) \exp -\left(\frac{x}{x_{R}}\right)^{2}
$$

converges to $C\left(x, t_{R}\right)$ as $N I \rightarrow \infty$ only if: ${ }^{19}$

$$
\left(2^{-1 / 2} x_{A}\right)<x_{R}<\infty
$$


Since oniy the first $\left(H_{0} \exp -\left(x / x_{R}\right)^{2}\right)$. term has a finite area under it, using $\int C d x=\hat{i}$, it requires $B_{0}=\hat{1} /\left(\pi^{1 / 2} x_{R}\right)$ and $\tilde{B}_{0}=\hat{1} /\left(\pi^{1 / 2} x_{F}\right)$. The convergence requirement is just a condition that prevents one from choosing the initial gaussian term in each series as too "skinny" compared to the average breadth of the profiles $c\left(x, t_{R}\right), i\left(x, t_{F}\right)$.

In practice, this convergence requirement is always satisfied as long as one seeks a reasonable data fit without an inordinate number of polynomials.

Finally, of course, data taken in the laboratory practically always presents $C(x, t)$ not as a continuous function but as a set of ordered pairs $\left[C\left(x_{i}, t\right), x_{i}\right]$ and the exact transformation from ordered pairs to a Hermite-polynomial series, given $x_{R}$ (or $x_{F}$ ), is presented in detail in Appendix III.

\section{Extracting Do}

Suppose now that the initial and final time concentration profiles were measured, the expansion parameters $x_{R}$ and $x_{F}$ chosen, and the orthogonal function series created. The quantities characterizing the initial state are: $\left[\left\{B_{p}, p=(0, N I)\right\}, x_{R}\right]$, and the final state: $\left[\left\{\tilde{B}_{p}, p=\right.\right.$ $\left.(0, N F)\} ; x_{F}\right]$. It is easiest to see the time development of the $c\left(x, t_{R}\right)$ state by inventing a $t_{R}^{*}$ and a set $\left\{\hat{A}_{2 p}\right\}$

$$
\begin{aligned}
& x_{R}{ }^{2}=4 D_{0} t_{R}^{*} \\
& \hat{A}_{2 p}=B_{p}\left(4 D_{0} t_{R}^{*}\right)(2 p+1) / 2
\end{aligned}
$$


then

$$
C_{R}\left(x ; t_{R}\right)=\sum_{p=0}^{N I}\left(\frac{\hat{A}_{2 p}}{\left(4 D_{0} t_{R}^{*}\right)}(2 p+1) / 2\right) H_{2 p}\left(\frac{x}{\sqrt{4 D_{0} t_{R}^{*}}}\right) \exp -\left(\frac{x^{2}}{4 D_{0} t_{R}^{*}}\right)
$$

This propagates forward until a time $t_{F}$ is reached (which is $\left(t_{F}-t_{R}\right)$ away from the $C_{R}\left(x, t_{R}\right)$ state) giving:

$$
\begin{aligned}
\hat{C}_{R}\left(x, t_{F}\right)= & \sum_{p=0}^{N I}\left(\frac{\hat{A}_{2 p}}{\left(4 D_{0}\left(t_{R}^{\left.\left.* x+\left[t_{F}-t_{R}\right]\right)\right)}(2 p+1) / 2\right) H_{2 p}\left(\frac{x}{x_{N}}\right) \exp -\left(\frac{x}{x_{N}}\right)^{2}\right.}\right. \\
x_{N}^{2} & =4 D_{0}\left(t_{R}^{* x+}\left[t_{F}-t_{R}\right]\right) .
\end{aligned}
$$

Re-expressing this in terms of $B_{p}$ and $x_{R}{ }^{2}$ gives:

$$
\begin{gathered}
\hat{C}_{R}\left(x, t_{F}\right)=\sum_{p=0}^{N I} B_{p}\left(x_{R} / x_{N}\right)^{2 p+1} H_{2 p}\left(x / x_{N}\right) \exp -\left(x / x_{N}\right)^{2} \\
\therefore \quad x_{N}^{2}=x_{R}{ }^{2}+4 D_{O}\left(t_{F}-t_{R}\right)
\end{gathered}
$$

This propagated forward state (denoted with a 'A') must then be compared with the measured final state $C_{F}\left(x, t_{F}\right)$.

At this point, we comment on the equation: $x_{N}{ }^{2}=x_{R}{ }^{2}+4 D_{0}\left(t_{F}-t_{R}\right)$. If limiting-slope were a valid determiner of $D_{0}$ for the linear diffusion equation, and if the (arbitrary) expansion parameters $x_{R}$ and $x_{F}$ were chosen to be related to the measured limiting slopes of the initial and final state as follows:

$$
\begin{aligned}
\text { L.S. (initial state) } & =-1 / \hat{x}_{R}^{2} \\
\text { L.S. (final state) } & =-1 / \hat{x}_{F}^{2}
\end{aligned}
$$

then one would have the relationship:

$$
\hat{x}_{F}^{2}=\hat{x}_{R}^{2}+4 \tilde{D}_{0}\left(t_{F}-t_{R}\right)
$$


as the generalization of the limiting-slope concept to a two time correlation, giving a limiting-slope diffusivity $\tilde{D}_{0}$ as discussed previously. If $\tilde{D}_{0}$ is indeed a valid measure of $D_{0}$ for the linear equation, then a simulation can be performed: starting with a given $D_{0}$, then propagating forward a time $\left(t_{F}-t_{R}\right)$, one can, by graphing the final state, pick off an "apparent-limiting-slope" and using Eq. (2.33), calculate $\tilde{D}_{O}$ and see if $D_{0}$ matches $\tilde{D}_{0}$. One finds they do not match in the first few decades. In general, since $D_{0}$ is a physical parameter, and $x_{R}$ and $x_{F}$ are arbitrarily chosen, one does not expect $x_{N}{ }^{2}=x_{F}^{2}$. Thus, the final step in the methodology, the isolation of the physical parameter $D_{0}$, is accomplished as follows:

Since the propagated-forward state $\hat{C}_{R}\left(x, t_{F}\right)$ has only one unknown in it, $D_{0}$, the difference between $\hat{C}_{R}\left(x, t_{F}\right)$ and the measured final state, $C_{F}\left(x, t_{F}\right)$ is minimized. In other words, one minimizes the following functional of $D_{0}$ :

$$
\Phi^{2}\left(D_{0}\right)=\int\left[C_{F}\left(x, t_{F}\right)-\hat{C}_{R}\left(x, t_{F}\right)\right]^{2} d x / \int\left[C_{F}\left(x, t_{F}\right)\right]^{2} d x
$$

The integrals in the minimizing functional can all be done exactly for they are all integrals of the following general form:

$$
I_{m, n}(a, b)=\int H_{2 m}(a \xi) H_{2 n}(b \xi) \delta\left(a^{2}+b^{2}=1\right) \exp ^{2} \xi^{2} \xi
$$

which has been solved by W.N. Bailey ${ }^{20}$ with the result:

$$
I_{m, n}(a, b)=(-1)^{m+n} \Gamma\left(m+n+\frac{1}{2}\right) 2^{2(m+n)} a^{2 n}{ }^{2 m}
$$

Since the denominator in each $\Phi^{2}$ is a fixed number (for normalization, so that $\dot{\phi}^{2}$ is a measure of fractional error) and independent of $D_{0}$, one 
then has the value of $D_{0}$ being determined by the global minimum of a polynomial expression in the variable $x_{N}$ with (as given before) $D_{0}$ determined from:

$$
x_{N}^{2}=x_{R}^{2}+4 D_{0}\left(t_{F}-t_{R}\right)
$$

Alternately, one could, by doing $\left(\frac{\mathrm{d}}{\mathrm{dD}_{0}}\right) \Phi^{2}=0$, reduce the problem to finding the root of a large polynomial, but that usually is no added simplification, and a search for a minimum of $\Phi^{2}$ in $D_{0}-\left(\right.$ or $\left.x_{N}\right)$-space suffices.

$$
* * *
$$

This completes the first "check" -- any given pair of data sets can now be tested to find the best linear equation diffusivity $D_{0}$. If the final state profile agrees with the propagated initial state profile, indeed then $D(C) \simeq D_{0}$ may be a sufficiently good approximation.

\section{Simulations}

One now has a full analytical' structure on which simulation studies can be performed. This involves simply picking a polynomial (times a gaussian) for an initial state, ascribing a time $t_{R}$ to it, choosing a diffusivity $D_{0}$ and a final time $t_{F}$, generating a final state, then by using the initial and final states, along with the physically releyant time parameter $\left(t_{F}-t_{R}\right)$, seeing if aforesaid analysis reproduces $D_{0}$. (it does).

By marking off specific points on these initial and final states, one can simulate discrete data $\left(C_{i}, x_{i}\right)$, and then apply the analysis as given in Appendix III to examine how close to the original function one 
can get, varying space between "data points," total number of points, decades of "data" taken, etc. One can add random noise to the data to simulate various additional types of errors. In general, one finds that the orthogonal function fitting procedure is quite insensitive to noise (to even levels approaching $10 \%$ noise, random) and that if data are chopped off at $x_{Q}$, then if $\varepsilon=$ fraction of the true "area" (representing diffusant amount) between $x_{Q}$ and $x=\infty, D_{0}$ may be incorrect by about a fraction $E$.

One can also examine the effect of using alternate values for $x_{R}$ and $x_{F}$ in re-creating the continuous data from the discrete pairs $\left(C_{i}, x_{i}\right)$. One finds of course, that neither $x_{R}$ nor $x_{F}$ can be "too small" (convergence problems, but before that sets in, too many polynomials are needed to specify a state, and the procedure in Appendix III starts requiring too many digits accuracy in the computations). More importantly, since there are" no mathematical difficulties associated with choosing $x_{R}$ or $x_{F}$ "too large," even though, again, many polynomials are needed to match the given state, one finds that because the discrete data end. after a final $x_{Q}$, a tail (usually the limiting slope function just extended to $\mathrm{N}$ decades, where $\mathrm{N}$ is a large number, usually 8 to 10 ) needs to be tacked on; to assure that in the entire range $x=\left(x_{Q}, \infty\right)$ where no data are specified, the procedure of Appendix III will avoid picking a fit which is very good in the data range $x=\left(0, x_{Q}\right)$. but which oscillates wildly in the range where no data are given: $\left(x_{Q}^{\prime}, \infty\right)$ :

We remind the reader that the nature of orthogonal function sets is that only the fundamental (first) member of the set has a finite area under it, and all other members of the set are oscillatory. The existence 
of a negative concentration, though unphysical in many laboratory situations, is not only mathematically necessary for the description of a state, but also in all diffusion equations (including non-linear ones), because one can replace $C$ by. $\left(C+C_{0}\right)$, where $C_{0}$ is a uniform fixed background concentration; at most altering the non-linear parameters in a c.d.d. equation (and leaving invariant, the linear equation), $c$ must be allowed to be an algebraic,(i.e., + or -) quantity. Even with a (monotonic) "tacked-on tail" to discrete data, the allowing of a negative concentration usually manifests itself as a few small oscillations in the 6 th to 10 th decade of the polynomial fit -- totally beyond the real data range and totally due to the finite number of polynomials (usually 4-8) used to fit each given $C(x)$ state.

By plotting the initial and/or final state on a $\left[\ln |C|\right.$ vs. $\left.x^{2}\right]$-graph we can, at any $x$, consider that point to be the "last" data point, and read off an apparent limiting-slope-diffusivity (using either $\hat{\mathrm{D}}_{0}$ or $\tilde{\mathrm{D}}_{\mathrm{O}}$ as outlined in Chapter 1 , section IV), which is then directly comparable to $\mathrm{D}_{0}$. One finds that the $\hat{\mathrm{D}}_{0}$ and $\tilde{\mathrm{D}}_{\mathrm{o}}$ (for this, the linear equation) so derived can be of $f$ by ten percent within the first few (2-4) decades (and an order of magnitude if one uses the first decade alone). One also finds that a direct comparison between $\dot{\vec{D}}_{0}$ or $\tilde{D}_{0}$ and $\mathrm{D}_{0}$ in the 6 th to 10th decade is often impossible because of axis crossings of the polynomial function, but a non-oscillatory profile would give $D_{0} \equiv \tilde{D}_{0}$.

Finally, by examining the "goodness of fit" of, successively, the $[1,2,3, \ldots N]$-polynomial fits to a data set, for a given $x_{R}$, one can find:

(1). First, the total area (total amount) is matched;

(2). Then, the extent (penetration) of the concentration profile; 
(3). Finally, the details of the shape of the profile;

(4). Rarely, if ever, is the apparent limiting slope matched in the first few decades.

This is not surprising, for the orthogonal function set, by inherently minimizing the functional:

$$
\Phi^{2}=\int\left[C\left(x, t_{R}\right)-\left[B_{m} H_{2 m}\left(\frac{x}{x_{R}}\right) \exp -\left(\frac{x}{x_{R}}\right)^{2}\right]^{2} d x / \int\left[C\left(x, t_{R}\right)\right]^{2} d x\right.
$$

is essentially minimizing error on a [C vs. $x]$-graph and not a [ $\mathrm{lnC}$ vs. $x^{2}$ ]-graph. That the solution to the diffusion equation results in a function set minimizing error on [C vs. $x$ ] is quite reasonable since the original diffusion equation is one involving $C, \nabla C$, and similar terms.

\section{Towards a Non-linear Equation Analysis}

Having presented a workable linear equation methodology for nongaussian profiles, there are two tests by which one can decide that this is sufficient (or not):

(1). In a two time propagation, the final state and the initial state propagated-forward should "agree"

(2). (A more severe test) Since $D_{0}$ should be a parameter independent of concentrations, diffusion time, etc., one could ask for the same value of $\mathrm{D}_{0}$ to handle a multiple-time correlation. For systems where a c.d.d. mechanism even weakly operates, one expects test (2) to fail eventually (in a sufficiently short-time limit). 
Given that a non-linear study may be necessary, we gather here some aspects of the linear equation calculation that will carry over to the non-linear one. As mentioned earlier (Chapter 1, section III), it is crucial that, whatever solution to a non-1inear equation is generated, it must allow for an arbitrary initial state at a finite time $t_{R}$. Thus, any analysis methodology must be capable of handling at least a set of parameters $\left\{B_{0}, B_{1}, \ldots B_{N 1}\right\}$, which are coefficients of some orthogonal function set for an initial state, and a similar second set $\left\{\tilde{B}_{0}, \tilde{B}_{1}, \ldots \hat{B}_{N 2}\right\}$ for a final state. In the limit of 1arge. N1 and N2, each set contains all the information that can be known about that state, so one should not need to handle any greater a complexity than that.

The essential two "pieces" of information the solution to a nonlinear equation can give are just:

(1). What set of orthogonal functions to use

(2). The means by which these orthogonal functions propagate in time. Because the equation to be considered is non-linear, it cannot be expected, as was with the linear equation, that each particular orthogonal function set member propagates uniquely into a cognate of itself as time progresses. Thus, this orthogonal-function-directed formulation must lead, in the nonlinear situation; to coupling between different members of the orthogonal function set. If a solution to the non-1inear problem is to be generated, some manner, therefore, must be found of decoupling this (non-linear) system, and that comprises, in principle, one of the major hurdles which must be overcome.

A large class of solutions to non-linear problems involves either. transforming the given non-linear equation to a linear one, ${ }^{21}$ or reducing 
a non-linear equation's solution to the solution of an associated linear equation $^{22}$ (many 'solition'. solutions are of this type). These do not seem easily applicable to $D(C)$ problems, nor is 1tnearization which returns one back to $\frac{\partial C}{\partial t}=D_{0}\left(\frac{\partial}{\partial x}\right){ }^{2} \mathrm{C}$. The general concentration dependent diffusion equation is of the form:

$$
\frac{\partial C}{\partial t}=\left(\frac{\partial}{\partial x}\right)^{2} F(C)
$$

where $F(C)$ can be practically anything.

The major thrust of the analyses presented here would fall in the "small expansion parameter" category, whereby writing

$$
D(C) \simeq D_{0}(1+\gamma f(C))
$$

one hopes that the $\gamma f(C)$ non-linear term can be 'small' compared to 1 in at least some cases of interest. Whereas, for many non-linear systems that have been studied, (especially those which are wave-like in nature) a small non-linearity parameter still does not guarantee against growth of non-linear terms (to the extent of overwhelming the linear contribution by orders of magnitude) and where bi- and multi-furcation (sudden appearance of multiple solutions) is common, 23 the fact that this system is totally diffusive in nature leads to the hope that being an on-going spreading-out and "smoothing" process, not only may a small-non-1inearparameter analysis be viable, but it may even be qualitatively accurate in the limit of the non-linearity parameter being not-quite-so-small.

We further expect that, given a model $\mathrm{D}(\mathrm{C}), \therefore$ as:

$$
\begin{aligned}
& D(C)=D_{0}\left(1+\alpha C+\beta C^{2}+\gamma C^{3}+\ldots\right) \\
& D(C)=D_{0}\left[\alpha C+\left(1+(\alpha C)^{2}\right)^{1 / 2}\right],
\end{aligned}
$$


since the parameters $\left(D_{0}, \alpha, \beta, \ldots\right.$ etc. $)$ are not known beforehand, but are determined from experimental data, the following structure will be preserved:

(1). A given initial state $C_{R}\left(x, t_{R}\right)$ propagates foward in time to a state $\hat{C}_{R}\left(x, t_{F}\right)$. The $\hat{C}_{R}\left(x, t_{F}\right)$ state will have in its specification all the physical parameters $\left(D_{0}, \alpha, \beta, \ldots\right)$

(2): The determination of the actual value of these parameters must come from choosing a point in the $\mathrm{K}$-dimensional hyperspace whose co-ordinates are the $\mathrm{K}$ different parameters: $\left(\mathrm{D}_{0}, \alpha, \beta, \ldots\right)$. This "operating point" will be chosen (similar to the linear equation) by minimizing the functional:

$\phi^{2}\left(D_{o}, \alpha, \ldots\right)=\int\left[C_{F}\left(x, t_{F}\right)-\hat{C}_{R}\left(x, t_{F}\right)\right]^{2} d x / \int\left[C_{F}\left(x, t_{F}\right)\right]^{2} d x$

or some cognate to it.

The formal non-linear analysis will begin two chapters hence, when the problem of the diffusivity, which varies as a linear function of concentration will be considered. In the immediate next chapter, we review some of the basic models which give rise to a c.d.d. problem in our particular system of interest: heterovalent (aliovalent) ion diffusion into strongly ionic solids. 


\section{CHAPTER 3}

\section{THE PHYSICAL ORIGINS OF A CONCENTRATION} DEPENDENT DIFFUSIVITY

\section{The 'Standard' $D(C)$ Model}

In this chapter, the following notation will be used:

(s): a member of a given defect species ' $S$ '

[s]: the local concentration of species ' $S$ '

$n(s)$ : the fraction of available sites occupied by: ' $S$ '

C : the local concentration of a specific heterovalent ion species (no brackets in notation)

$\left[\mathrm{s}_{\mathrm{o}}\right]$ : the local concentration of ' $\mathrm{s}$ ' when $\mathrm{C} \rightarrow 0$

$\mathrm{n}_{\mathrm{o}}(\mathrm{s}): \therefore$ the fraction of available sites occupied by ' $\mathrm{S}$ ' when $C \rightarrow 0$

$(v+)$ : a positive ion or cation vacancy (negative effective charge)

$(v-)$ a negative ion or an ion vacancy (positive effective charge)

(d): a divacancy: a bound $\left(v^{+}\right),(v-)$ pair

$i:$ 'proportional to' (in contrast to $\simeq$ 'approximately equals')

The development of a concentration dependent diffusivity; being a nonlinearity, must arise from some fundamental self-coupling. In many brittle (cleavable) substances, the elastic strain energy is too great 
to allow for easy interstitial migration. In these systems, the primary atomic migration occurs via the vacancy mechanism, whereby a given atom can move only if a vacancy is nearby on its sublattice. Furthermore, if a diffusing ion is approximately the size of (or larger than) the host atom closest to its charge, it will tend to enter substitutionally. The large lattice elastic strain energy is also a direct consequence of either a definite ionicity or strong covalency of the interatomic bonds, both giving very few free electrons. Thus, any local charge imbalance is not capable of being easily. electron-screened (as would be in a metal). For metallic ion diffusion in brittle ionic crystals, one then has the two basic assumptions:

(1). $D \sim[v+] . \quad($ or $D=\lambda[v+]$, vacancy mechanism)

(2). Local charge neutrality is rigorously maintained. Assumption-2 is a consequence of both lack of shielding as well as an implicit assumption that whatever processes which could create a charge imbalance are quickly neutralized. This, in turn, is used in two ways:

(A). If a heterovalent (impurity) ion is introduced into these lattices, the charge mismatch is initially compensated for by the formation of extra ion vacancies on the sublattice in which the impurity ion is situated to maintain a local charge balance.

For a charge mismatch of m-units, where $m=\left(q-q_{0}\right) / q_{0}, ' q^{\prime}$ being the heterovalent ionic charge and $q_{o}$ the host sublattice charge (m may be fractional), an average of $(1+m)$ defects are thus created.

(B). Lacking interstitials, the ion vacancies being a Shottkylike disordered system (vacancies on $(+)$ and $(-)$ ion 
sublattices), only groups of net neutral charge may be created or annihilated (at, for example, grain boundaries, dislocations, etc.)

Using $\left[v_{0}\right]$ for the concentration of $(+)$ or $(-)$ ion vacancies in the $C \rightarrow 0$ limit, an introduction of a concentration ' $C$ ' of impurity of charge mismatch ' $m$ ' gives:

$$
[v+]=\left[v_{0}\right]+m C
$$

and with $D_{0}$ the limiting concentration diffusivity $\left(D_{0}=\lambda\left[v_{0}\right]\right)$ one has:

$$
D(C)=\lambda[v+]=D_{0}[v+] /\left[v_{0}\right]=D_{0}(1+K) ; K=m C /\left[v_{0}\right]
$$

Thus, even in this simplest of all models, a concentration dependent diffusivity is obtained. The self-coupling, obviously, is that the mass transport of the species ' $C$ ', governed by a $D$. depending on $[v+]$, has the $[v+]$ parameter itself altered by the presence of ' $C$ '.

Given the inherent Shottky-like disorder, and because the introduction of ' $C$ ' has so far altered only one of the defect concentrations, usually an additional process of equilibration of some vacancy-vacancy interaction is assumed. The most common ("standard") model 24,25 assumes that the interaction of vacancies with extended defects such as dislocations (line-1ike) and domains, grain-boundaries, and crystal edges (surface-like) is in equilibrium. Specializing to compounds of stoichiometry (Metal) ${ }_{1}$ (Non-metal) $_{1}$ which exhibit Shottky disorder, this imposes the additional constraint:

$$
n(v+) n(v-)=\underline{K}_{0}(T)=n_{0}(v+) n_{0}\left(v^{-}\right) \equiv n_{0}^{2}
$$

where $\underline{K}_{0}$ is an equilibrium constant (usually written $\underline{-}_{0}=\operatorname{exp-G} F / k T$ where $G^{F}$ is the (Gibbs) Free-energy of formation for the Shottky pair) 
and $n_{0}(v+)=n_{0}(v-)$ (the fraction of sites occupled by ( $v+$ ) equalling those occupied by $(v-)$ in the absence of impurities $(C \rightarrow 0)$, also giving an assignment of $\mathrm{G}^{\mathrm{F}} / 2$ as the free-energy of formation of either a single $(v+)$ or $(v-)$ defect). Explicit charge neutrality gives:

$$
[v+]=[v-]+m C \text {. }
$$

Writing the equilibrium constraint alternately as:

$$
[v+][v-]=\left[v_{0}\right]^{2}
$$

and solving for $[\mathrm{v}+]=\mathrm{f}\left(\left[\mathrm{v}_{\mathrm{o}}\right], \mathrm{mC}\right)$, the standard modei then gives:

$$
\mathrm{D}(\mathrm{C})=\mathrm{D}_{\mathrm{o}}[\mathrm{v}+] /\left[\mathrm{v}_{\mathrm{o}}\right]=\mathrm{D}_{\mathrm{o}}\left((\mathrm{K} / 2)+\left[1+(\mathrm{K} / 2)^{2}\right]^{1 / 2}\right) ; \mathrm{K}=\mathrm{mC} /\left[\mathrm{v}_{\mathrm{o}}\right]
$$

The major difference between this and the previous (no-equilibrium constraint) model is that in the approximation of $D(C)$ as a linear function of ' $C$ ' (for low $C$ ), the coefficient of $K$ in this model is smaller by a factor of 2 :

A caution on notation: Beginning with sec. III of this chapter, a new (expanded) notation will be introduced; and the equations of this section, in the new notation, will be:

$$
\begin{aligned}
& {[\hat{\mathrm{v}}+][\hat{\mathrm{v}}-]=\left[\hat{\mathrm{v}}_{0}\right]^{2}} \\
& {[\hat{\mathrm{v}}+]=[\hat{\mathrm{v}}-]+\mathrm{mC}, \hat{\mathrm{K}}=\mathrm{mC} /\left[\hat{\mathrm{v}}_{\mathrm{o}}\right]} \\
& \left.\mathrm{D}(\mathrm{C})=\mathrm{D}_{\mathrm{o}}[\hat{\mathrm{v}}+] /\left[\hat{\mathrm{v}}_{\mathrm{o}}\right]=\mathrm{D}_{\mathrm{O}}(\hat{\mathrm{K}} / 2)+\left[1+(\hat{\mathrm{K}} / 2)^{2}\right]^{1 / 2}\right) \\
& \therefore * *
\end{aligned}
$$




\section{The $n(v+) n(v-)=K_{0}(T)$. Constraint}

Next; carefully examining the restriction $n\left(v^{+}\right) n\left(v^{-}\right)=K_{0}(T)$, preservation of this condition requires a local change of both anion and cation vacancy concentrations. The usual plausibility argument offered 26 is that the internal extended defects serve as infinite sources or sinks of vacancies, and as such, they maintain this thermodynamic equilibrium "locally". Thus, this is a model which has a local equilibrium constraint on the concentration of one defect type (vacancies) determining the diffusivity of another defect type (the impurity. ' $\mathrm{C}$ '). Because the diffusion equation of the impurity ' $C$ ' is being explicitly considered, it necessitates that ' $C$ ' must not be in equilibrium. Therefore, one must have $D(v+) \geq D\left(v^{-}\right)$. $\gg D(C)$. If this inequality were not strongly satisfied, one would then have to consider coupled diffusion equations, one diffusion equation for each species: (v+), (v-), (C). Certainly, $D(v+) \gg D(C)$ since the motion of ' $C$ ' necessitates the presence of a $(v+)$, and one further expects $D(C) \sim n(v+) D(v+)$, with $n(v+) \sim 10^{-5}$ in alkali halides? 4,27 The question of whether $D(v-) \gg D(C)$ is satisfied, is more difficult. The ratio $D(v+) / D(v-)$ (as measured by the self-diffusion ratio, for example, $D(\mathrm{Na}) / \mathrm{D}(\mathrm{C} \ell)$ in $\mathrm{NaCl}$ ) has a wide range of values reported in the literature, ${ }^{28-30}$ mainly due to complications arising from the increasing role of dislocation diffusion and boundary diffusion for anions with inherently slow bulk diffusivities.

$$
* * *
$$

Whereas $D\left(v^{+}\right) \geq D\left(v^{-}\right) \gg D(C)$ is a restriction involving three 'bulk' quantities, another difficulty with this 'standard' model is that 
it is hinging the bulk equilibrium (of $\left(v^{+}\right)$and $\left(v_{-}\right)$) on a vacancy-surface interaction, or on a vacancy-line interaction. This model requires the continual adsorption and desorption of vacancies at grain boundaries or crystal edges (surfaces), and at dislocations (lines). That this leads to difficulties is elucidated by the following example:

As a model of domain wall sorpters, consider a cube of $\mathrm{N}^{3}$ atoms, whose entire surface is modeled as trapsites. This gives $6 \mathrm{~N}^{2}$ trapsites bounding the $\mathrm{N}^{3}$-atom domain, each surface trapsite being shared by two such domains, giving $3 \mathrm{~N}^{2}$ trapsites per domain (a 3 p.p.m. concentration of such domain wall trapsites tessallates a crystal into cubes $10^{6}$ atoms on a side).

Vacancies interior to each region (even though the total concentration of trapsites is $3 / N$ ) must travel on the average (N/2) lattice units before they interact with the domain wall; requiring about $(\mathrm{N} / 2)^{2}$ hops.

Next, as a model of uniformly distributed point-defect sorpters, consider a subunit cube of $\mathrm{N}^{3}$ atoms whose corners are now considered trapsites. This gives 8 trapsites in a subunit, each trapsite shared by 8 such subunits, giving 1 trapsite per subunit (a 3 p.p.m. concentration of point-defect trapsites tessallates a crystal into "cubes" of approximately $69 \times 69 \times 70$ atoms). Vacancies interior to these regions (with a trapsite concentration $1 / \mathrm{N}^{3}$ now require about $\mathrm{N}^{3}$ hops before interacting with a trapsite. Notice that in the point-defect case, the average number of hops scales (as a function of $N$ ) with the inverse concentration, whereas for the domain-model; the exponent of the average number of hops (before reaching a trapsite) exceeds the exponent of the inverse 
concentration. This is because these trapsites, which need to be coherent (in space) to form a domain wall, lessen the chance of an interior point defect interacting with them.

And, finally, as a model of dislocation sorpters, consider a cube of $\mathrm{N}^{3}$ atoms whose edges are now trapsites. This gives $12 \mathrm{~N}$ trapsites in a subunit, each edge shared by 4 such subunits, giving $3 \mathrm{~N}$ trapsites per subunit (a 3 p.p.m. concentration of dislocation trapsites would tessallate a crystal into cubes 1,000 atoms on a side). Vacancies interfor to these regions (whose total concentration of trapsites is $3 / \mathrm{N}^{2}$ ) we now model as requiring $\sim \mathrm{N}^{\mathrm{K}}$ hops before interacting with a trapsite, where one expects $2<\mathrm{K}<3$. For "small" $\mathrm{N}$ (1arge dislocation densities) the distance to travel across a subunit becomes less important compared to total number of sites occupied by trapsites, and one expects the in-. verse concentration to be a good approximate measure of the average number of hops (i.e., one requires $\sim N^{2} / 3$ hops). For very large $N$ (small dislocation density) one requires $\sim(\mathrm{N} / 2)^{2}$ hops to reach the subunitcube border, but only $(3 / N)$ of the totality of surface and edges of this cube are trapsites, thus, requiring roughly $\sim 3 \mathrm{~N}^{3} / 4$ hops before interacting with a trapsite.

A measure of equilibration time $\tau$ in each of these three cases, given a vacancy hopping frequency $\nu_{0}$, is $\tau=f(N) / \nu_{0}$; where $f(N)$ is some average number of hops before a trapsite interaction. For the same global concentration of trapsites in each of these three cases, with $\mathrm{Na}{ }_{0}=$ domain or subunit size ( $\mathrm{la}_{\mathrm{O}}=1$ lattice distance), remembering that 
equivalent concentrations tessallate a crystal into different subunit sizes, one has:

$$
\begin{aligned}
& \text { Trapsite } \\
& \text { type. }
\end{aligned}
$$

Domain wall

Dislocation

Point-defect
Trapsite concentration $\tau(\mathrm{n}):$
$n=3 / N$
$\tau=\mathrm{N}^{2} / 4 v_{0}$
$\tau \sim 1 / n^{2} v_{0}$
$\mathrm{n}=3 / \mathrm{N}^{2}$
$\tau \simeq\left(N^{K} / \nu_{0}\right)$
$(2<K<3)$
$\tau \sim 1 / \mathrm{n}^{\mathrm{K} / 2} \nu_{\mathrm{o}}$
$n=1 / N^{3}$
$\tau=N^{3} / \nu_{0}$
$\tau \sim 1 / \mathrm{nv}{ }_{0}$

Using $\tau$ (dislocations) $\sim \mathrm{N}^{3} / \nu_{0}$ (the low concentration limit) one has, for example, at 3 p.p.m. trapsite concentration

$\tau\left(\right.$ point-defects): $\tau($ dislocations $): \tau($ domain walls $):: 1: 577: 3.33 \times 10^{5}$

Thus, not only is any vacancy-extended defect interaction significantly slower in equilibration than a vacancy-point defect interaction, but also when these characteristic times to equilibrate with extended defects (which naturaliy: tessallate the lattice into regions of size $\mathrm{Na}{ }_{0}$ ) become on the order of the time for an aliovalent diffusing ion to cross this region (of size $\mathrm{Na}_{0}$ ), the assumed equilibration constraint on vacancy concentration must be strongly violated.

$$
* * *
$$

Some further difficulties with this standard model are as follows: The adsorption or desorption of vacancies creates a volume charge locally within each domain or subunit, which could introduce a significant amount of local strain, thereby affecting defect motion. Furthermore, not only can this process not proceed indefinitely in one direction (whether it is volume increase or volume decrease), but also this process has the effect 
of accentuating polycrystallinity in any given sample. Another difficulty is that even if an extended defect could absorb or create an arbitrary amount of vacancy pairs, it does not imply that the extended defect is a perfect absorber of vacancies incident upon it. This has the effect of increasing the effective distance between these extended defects, or equivalently increasing, even further, the equilibration time.

And, finaliy, a large extended defect concentration can significantly channel ion motion so that atomic migration along these extended defects can become comparable to the bulk diffusion. 31

We, therefore, suggest that the primary thermodynamic equilibrium of vacancies is not the $(v+)+(v-) \longleftrightarrow$ (extended defects) interaction, but rather the defect-defect interaction $(v+)+(v-) \longleftrightarrow$ (d) where (d) is a divacancy. This process, as a bulk interaction, would be operative irrespective of extended defect concentrations, and being a bulk equilibrium involving point defects, it equilibrates faster than an equilibrium involving extended defects. To first order, it does not have a large volume change associated with it, nor does it require $D\left(v^{-}\right)>$. $D(C)$ as a necessary condition. In the next section, the analogues to the 'standard' model are developed, except that $(v+)+(v-) \leftrightarrow$ (d) will be used as the fundamental vacancy equilibration process.

\author{
* * * \\ III. The $(v+)+(v-) \longleftrightarrow$ (d) Process
}

As mentioned earlier, the diffusion equation for an aliovalent ion, whereby $\mathrm{D}(\mathrm{C})$ is determined by a vacancy equilibrium process, sets 
a natural upper bound on the equilibration time for such vacancy processes for the D.(C) concept to be valid. This natural time scale divides all defect interactions into 3 classes:

(1): 'Fast processes' which can be considered always in local thermodynamic equilibrium

(2): 'Slow processes' whose equilibration times are sufficiently long that they may be neglected

(3): 'Equivalent processes' which are in non-equilibrium, with equilibration times of the same order as the main diffusive process under consideration.

In general, class-3 type problems are very difficult to handle for they would require consideration of coupled diffusion equations with practically every species' concentration and concentration gradient being important. Therefore, for the remainder of this thesis the class-3 situation will be bypassed, for reasons of difficulty, rather than whether it could be the fundamental experimental situation at hand.

We, thus, consider the two processes $n(v+) n(v-)=\underline{K}_{0}(T)$ and $\left(v^{+}\right)+\left(v^{-}\right) \longleftrightarrow(d)$ as processes in class -1 or class -2 only. We examine both simultaneously, so that, in the proper limit, this further model will reduce to the 'standard' one. An additional notation' is also introduced: Whereas the $\mathrm{n}(\mathrm{v}+) \mathrm{n}(\mathrm{v}-)=\mathrm{K}_{\mathrm{o}}(\mathrm{T})$ mass-action law does not distinguish between 'bound' and 'free'vacancies, the $(v+)+\left(v^{-}\right) \longleftrightarrow$ (d) reaction does, the (d) being a 'bound' vacancy state. We, henceforth, reserve $n(v),[v]$ for free vacancies of the type $(v)$; and $\hat{n}(v),[\hat{v}]$ for the total (free + bound) of the type $(v)$. One then has

$$
\hat{n}(v+) \hat{n}(v-)=\underline{K}_{0}(T)=\hat{n}_{0}(v+) \hat{n}_{0}(v-) \equiv \hat{n}_{0}^{2}
$$


for the vacancy-extended defect equilibrium equation. A second equilibrium constraint. $\underline{K}_{1}(T)$ is introduced for the reaction:

$$
(v+)+(v-) \longleftrightarrow(d)
$$

and following standard mass-action principles (1st order chemical kinetics),

$$
\underline{\mathrm{k}}_{1}(\mathrm{~T})=\mathrm{n}(\mathrm{v}+) \cdot \mathrm{n}(\mathrm{v}-) / \mathrm{n}(\mathrm{d})=\mathrm{n}_{\mathrm{o}}(\mathrm{v}+) \mathrm{n}_{\mathrm{o}}(\mathrm{v}-) / \mathrm{n}_{\mathrm{o}}(\mathrm{d}) \equiv \mathrm{n}_{\mathrm{o}}^{2} / \mathrm{n}_{\mathrm{d}}
$$

where $n_{0}$ (with no added specification) $=$ free $(+)$ or $(-)$ ion vacancy fraction-of-sites occupied as $C$ (impurity) $\rightarrow 0$, and where $n_{d}$ is the intrinsic divacancy concentration in this $\mathrm{C} \rightarrow 0$ limit.

In going from the simple situation of vacancy interactions governed by $\underline{K}_{0}(T)$ to this extended model with both $\underline{K}_{0}(T)$ and $\underline{K}_{1}(T)$ operating, $\underline{K}_{0}(T)$ was chosen to be the product of the total $(+)$ and $(-)$ ion vacancy fractional-site-occupations, instead of the free fractional-site-occupations. This choice is justified in this manner: The original $" n(v+) n(v-)=$ $\underline{K}_{0}(T) "$ (i.e., the 'standard' model wïth no further detail and not addressing the divacancy question) reflects the interaction:

$$
(v+)+(v-)+\longrightarrow[,]
$$

the blank brackets [ ] representing interaction with an infinite source or sink of vacancies. One could have, instead, considered the two reactions:

$$
\begin{aligned}
& (v+) \stackrel{\longleftrightarrow}{\longleftrightarrow}] ; \underline{K}_{A}=\exp -\left(G^{A}\right) / k T \\
& (v-) \stackrel{\leftrightarrow}{\longleftrightarrow}[\quad] ; \underline{K}_{B}=\exp -\left(G^{B}\right) / k T
\end{aligned}
$$

where $\left(G^{A}\right)$ and $\left(G^{B}\right)$ now represent the free-energy of formation of a single vacancy, and where $\left(G^{A}\right)+\left(G^{B}\right)=\left(G^{F}\right),\left(G^{F}\right)$ being the (pair) free-energy of formation, instead of proceeding with the assignment of $\left(G_{F} / 2\right)$ to each member of the Shottky defect pair. 
The reason this formalism would not be applicable in this case (vacancies in strongly lonic crystals) is that by writing a $\underline{k}_{A}$ and a $\underline{\mathrm{K}}_{\mathrm{B}}$ (two separate equilibrium constraints) one is implying that $(\mathrm{v}+$ ) and $(v-)$ formation are totally (thermodynamically) decoupled. But charge neutrality strongly couples the concentrations, to a point overwhelming the individual formation energies, and this is the reason that a single equilibrium constant $\underline{K}_{0}(T)$ is correct. To try to maintain a $\left(\underline{K}_{A}(T)\right)$ and $a\left(\underline{K}_{B}(T)\right)$. independently is an over specification and leads to a logical contradiction.

Consider next the two processes:

$$
\begin{gathered}
(v+)+\left(v^{-}\right) \longleftrightarrow[\quad] \text { (surface interaction); } \\
n n(v+) n\left(v^{-}\right)=K_{0}(T)^{\prime \prime} \\
\vdots\left(v^{+}\right)+\left(v^{-}\right) \longleftrightarrow \text { (d) (bulk interaction); } \\
n\left(v^{+}\right) n\left(v^{-}\right) / n(d)=\underline{K}_{1}(T) .
\end{gathered}
$$

Interpreting $n(v t), n(v-)$ as free vacancy concentrations in both $\mathrm{K}_{0}(T)$ and $\underline{K}_{1}(T)$ makes $n(d)$ unable to change (i.e., $n(d)=K_{0}(T) / K_{1}(T)$ ), and thus it would be more appropriate for the reactions:

$$
\begin{aligned}
& (\mathrm{v}+)+(\mathrm{v}-) \longleftrightarrow[\text { [ (surface interaction) } \\
& (\mathrm{d}) \longleftrightarrow[\mathrm{l} \text { (surface interaction). }
\end{aligned}
$$

It may be argued that Eq. (3.14a) refers only to free vacancy sorption at surfaces; then ( $3.14 \mathrm{~b}$ ) is necessarily an allowed independent reaction (whereas if Eq. (3.14a) (or Eq. 3.13a) is viewed as referring to the total of free and bound vacancies, then Eq. (3.14b). is redundant). This is a possible dynamical situation, but it does not respect $(v+)+(v-) \longleftrightarrow(d)$ as a bulk equilibration process. Thus, similar to the choice of $\underline{K}_{0}(T)$ 
(over $\underline{K}_{A}(T)$ and $\underline{K}_{B}(T)$ ). so that one may model the coupling between ( $\mathrm{v}+$ ) and $(v-)$ reflected in the necessity of total charge neutrality, one must choose $\hat{n}(v t) \hat{n}(v-)=\underline{K}_{0}(T)$ (over $n(v t) n(v-)=\underline{K}_{0}(T)$ ), so that one may model the bulk reaction $(v+)+\left(v^{-}\right) \longleftrightarrow$ (d) instead of being confined entirely to vacancy-surface interactions.

Thus, with the reactions:

$$
\begin{aligned}
& \hat{n}(v+) \hat{n}(v-)=\underline{K}_{0}(T)=\hat{n}_{0}^{2} \\
& n(v+) n(v-) / n(d)=\underline{K}_{1}(T)=n_{0}^{2} / n_{d}
\end{aligned}
$$

one has 2 cases for consideration.

CASE 1: The Perfect Crystal Limit. $\hat{n}\left(v^{+}\right) \hat{n}\left(v^{-}\right)=K_{0}(T)$ is treated as a 'slow' process, and is neglected. $(v+)+(v-) \longleftrightarrow$ (d) is treated as a 'fast' process. The introduction of a concentration $C$ of heterovalent ions with a charge mismatch $\mathrm{m}=\left(\mathrm{q}-\mathrm{q}_{0}\right) / \mathrm{q}_{0}$ (as defined in sec. I) introduces a sudden change, in the concentration of $\left(v^{+}\right)$:

$$
[v+]=\left[v_{0}\right]+m C
$$

Re-equilibration creates one new divacancy for each ( $(v+)$, (v-) pair annihilated:

$$
\underline{K}_{1}(T)=\left(n_{0}+m n(C)-s\right)\left(n_{0}-s\right) /\left(n_{d}+s\right)=n_{0} 2 / n_{d}
$$

where ' $s$ ' is the additional fraction of sites occupied by 'new' divacancies. In terms of $[v+],\left[v_{0}\right],\left[d_{0}\right],[c]$, this is:

$$
[\mathrm{v}+]([\mathrm{v}+]-\mathrm{mC}) /\left(\left[\mathrm{d}_{0}\right]+\left[\mathrm{v}_{0}\right]+\mathrm{mC}-[\mathrm{v}+]\right)=\left[\mathrm{v}_{0}\right]^{2} /\left[\mathrm{d}_{0}\right]
$$

Using $\left[\mathrm{v}_{0}\right]+\left[\mathrm{d}_{0}\right]=\left[\hat{\mathrm{v}}_{\mathrm{o}}\right]$, and defining $\mathrm{p}=\left[\mathrm{v}_{\mathrm{o}}\right] /\left[\mathrm{d}_{0}\right]$, this gives

$$
[v+]^{2}-m C[v+]=p\left[v_{0}\right]\left(\left[\hat{v}_{0}\right]+m C-[v+]\right) \text {. }
$$


Since $\left[v_{0}\right]+m C>[v+]>\left[v_{0}\right]$, it results in:

$$
2[v+]=\left[\left(p\left[v_{0}\right]+m C\right)^{2}+4 p\left[v_{0}\right]\left[\hat{v}_{0}\right]\right]^{1 / 2}-\left(p\left[v_{0}\right]-m C\right) .
$$

And, finally, with $\mathrm{p}\left[\hat{\mathrm{v}}_{\mathrm{o}}\right]=\left[\mathrm{v}_{\mathrm{o}}\right](1+\mathrm{p})$, one has:

$$
2[\mathrm{v}+]=\left[\left(\mathrm{p}\left[\mathrm{v}_{0}\right]+\mathrm{mC}\right)^{2}+4(1+\mathrm{p})\left[\mathrm{v}_{0}\right]^{2}\right]^{1 / 2}-\left(\mathrm{p}\left[\mathrm{v}_{0}\right]-\mathrm{mC}\right)
$$

The divacancy concentration is easily found from $[\mathrm{d}]=\left[\hat{\mathrm{v}}_{0}\right]-[\mathrm{v}+]+\mathrm{mC}$. In terms of $\mathrm{K}=\mathrm{mC} /\left[\mathrm{v}_{\mathrm{o}}\right]$ :

$$
[v+] /\left[v_{0}\right]=\left[(1+p)+(K+p)^{2} / 4\right]^{1 / 2}+[(K-p) / 2] .
$$

There are two special limits of interest. The first, $p>>1$ (very few divacancies), must be similar to a model where each impurity adds exactly one extra $(v+)$, and indeed

$$
\begin{aligned}
& {[\mathrm{v}+] /\left[\mathrm{v}_{0}\right] \simeq(1+K)-[K / \mathrm{p}]+\left[K(2+K) / \mathrm{p}^{2}\right]+\ldots} \\
& {[\mathrm{d}] /\left[\mathrm{v}_{0}\right] \simeq[(1+K) / \mathrm{p}]-\left[K(2+K) / \mathrm{p}^{2}\right]+\ldots}
\end{aligned}
$$

The other limit, $p \ll 1$, gives (neglecting terms of order $\mathrm{p}^{4}, \mathrm{k}^{4}$ ):

$$
\begin{aligned}
& \mathrm{f}_{1}(\mathrm{p}, \mathrm{K}) \stackrel{\doteq}{=}\left[1-(\mathrm{K} / 4)-\left(\mathrm{K}^{2} / 8\right)\right]-.5\left[1+\left(3 \mathrm{~K}^{2} / 8\right)\right] \mathrm{p}+.25[1+(5 \mathrm{~K} / 4) \\
& \left.+\left(5 K^{2} / 8\right)\right] p^{2} \ldots \\
& {[\mathrm{v}+] /\left[\mathrm{v}_{\mathrm{o}}\right]=1+.5[1+(\mathrm{K} / 4)] \mathrm{K}+.25 \mathrm{Kp} \mathrm{f}_{1}(\mathrm{p}, \mathrm{K})+\ldots} \\
& {[d] /\left[v_{0}\right] \rightleftharpoons(1 / p)+.5[1-(K / 4)] K-.25 K p \cdot f_{1}(p, k)+\ldots}
\end{aligned}
$$

The particularly interesting quality of this $p \ll 1$ limit, easily seen from the full analytic expression for $[\mathrm{v}+] /\left[\mathrm{v}_{0}\right]$ (Eq. 3.19), is that as $p \rightarrow 0$ this model exactly reduces to the functional form of the standard model (the only difference being a $\mathrm{K}=\mathrm{mC} /\left[\mathrm{v}_{0}\right]$ in this model, and a $\hat{\mathrm{K}}=\mathrm{mC} /\left[\hat{\mathrm{v}}_{\mathrm{o}}\right]=\mathrm{p} /\left(\left[\mathrm{v}_{\mathrm{o}}\right](1+\mathrm{p})\right)$ in the standard model). The reason for this 
is clear: Even though the $p \rightarrow 0$. 1imit is a high-divacancy concentration -situation, as long as $D \sim[v+]$ is chosen, the divacancy is viewed as a trapsite absorbing ( $v+$ ) which removes it from the diffusion process. (the cognate to extended defects being trapsites in the 'standard' mode1). The equilibrium concentration is functionaliy the same because whereas the 'standard' model requires extended defects to act as infinite sinks or sources of vacancies, this model uses the divacancy point-defect as a sink or source of vacancies; and (only) in the $p \rightarrow 0$ limit (extreme high divacancy concentration), can the divacancies act as an infinite supply (or sink).

CASE 2: The polycrystaliine limit. Both $\hat{n}(v+) \hat{n}(v-)=\underline{K}_{0}(T)$ and $(\mathrm{v}+)+(\mathrm{v}-) \longleftrightarrow$ (d) are treated as important. Using the same definitions of $p ; K$ as before, one has the two independent equations:

$$
\begin{aligned}
& [\hat{v+}] \cdot \hat{v}-]=\left[\hat{v}_{0}\right]^{2} \\
& {[v+] \cdot[v-]=p\left[v_{0}\right][d] .}
\end{aligned}
$$

The addition of a concentration $\mathrm{C}$ of aliovalent ions gives:

$$
[\mathrm{v}+]-[\mathrm{v}-]=m C=[\hat{\mathrm{v}}+]-[\hat{\mathrm{v}}-]
$$

Using (Eq. 3.26) with (Eq. 3.25b) results in:

$$
[\mathrm{v} \pm]=\left[(\mathrm{mC} / 2)^{2}+\mathrm{p}\left[\mathrm{v}_{\mathrm{o}}\right][\mathrm{d}]\right]^{1 / 2} \pm(\mathrm{mC} / 2)
$$

and using (Eq. 3.25a) which now becomes:

$$
\left[\hat{\mathrm{v}}_{\mathrm{o}}\right]^{2}=\left(\left[(\mathrm{mC} / 2)^{2}+\mathrm{p}\left[\mathrm{v}_{\mathrm{o}}\right][\mathrm{d}]\right]^{1 / 2}+[\mathrm{d}]\right)^{2}-(\mathrm{mC} / 2)^{2}
$$

the following quadratic for [d] is found:

$$
\begin{aligned}
& b\left[v_{o}\right]=\left(\left[\hat{v}_{o}\right]^{2}+(m c / 2)^{2}\right)^{1 / 2} \\
& 0=[d]^{2}-(2 b+p)\left[v_{o}\right][d]+\left[\left(b\left[v_{o}\right]\right)^{2}-(m c / 2)^{2}\right]
\end{aligned}
$$


Note: If this set of steps is not used, one may end up with a Quartic equation for [d].

Defining $E=[d] /\left[v_{0}\right]$ (and as $\left.C \rightarrow 0, E \rightarrow 1 / p\right)$, one has

$$
\begin{aligned}
& b=\left[\left(1+p^{-1}\right)^{2}+(K / 2)^{2}\right]^{1 / 2} \\
& E^{2}-(2 b+p) E+\left(b^{2}-(K / 2)^{2}\right)=0
\end{aligned}
$$

For small impurity concentrations, a series expansion near $\mathrm{K}=0$ give:

$$
\begin{aligned}
& {[\mathrm{d}] /\left[\mathrm{v}_{\mathrm{o}}\right] \equiv E=(1 / \mathrm{p})-\left[\left(\mathrm{K}^{2} /(4(\mathrm{p}+1)(\mathrm{p}+2))\right]+\operatorname{order}-\left(\mathrm{K}^{3}\right)\right.} \\
& \left.[\mathrm{v} \pm] /\left[\mathrm{v}_{0}\right]=1 \pm .5 \mathrm{~K}+.125[1-\mathrm{p} /(\mathrm{p}+1)(\mathrm{p}+2))\right] \cdot \mathrm{K}^{2} \ldots
\end{aligned}
$$

Two noteworthy points: The first three terms of the $[v \pm] /\left[v_{0}\right]$ equation functionally match the 'standard' model (especially note that the coefficient of the order $(K)$ is independent of $p$, which is not true for Case-1) except for the divacancy contribution as measured by $p$ correcting the $k^{2}$-term. This correction is $p /(p+1)(p+2)$; the maximum value of this occuring at $p=2^{1 / 2}$ with a value:

$$
\max [\mathrm{p} /((\mathrm{p}+1)(\mathrm{p}+2))]=.17157^{\circ}
$$

Thus, In this Case-2 situation (as in Case-1 where the $p=0$ limit gave a:-functional form identical to the standard mode1), the concurrence with the standard model in the $p \rightarrow 0$ limit is not because the physical processes giving rise to the $[\mathrm{v}+] /\left[\mathrm{v}_{0}\right]$ equation become concurrent (they don't) but, rather it is because the forced condition of equilibrium creates the similarities in the $[\mathrm{v}+] /\left[\mathrm{v}_{0}\right]$ functions. In other words, very similar equations are obtained from very different physics. 
Since (especially in a $D \sim[v+]$ model) the $[v+] /\left[v_{0}\right]$ function will go directly into the diffusion equation as a D(C) process; one has the following:

(1). A model using $D(C)=D_{0}(1+\alpha C)$, with $\alpha=$ non-linearity parameter,will be able to measure the effect of going from a linear equation to a first non-linear correction, and, in a sense, will measure the degree of non-linearity present in the system; however, as both phenomenological parameters $\left(D_{0}, \alpha\right)$ are determined from experiment, one cannot distinguish between a no-thermodynamics model, the 'standard' model, or any of the further models presented here, by using just the linear functional of concentrations.

(2). To begin to differentiate between these various physical models, one needs to consider at least $D(C)=D_{0}\left(1+\alpha C+\beta C^{2}\right)$, and, even then, even given a solution to this more complicated problem, one further needs the solution methodology to be sensitive to the value of $\beta$, otherwise, again, a distinction between different physical models cannot be made with confidence.

To restate: For the existence of a c.d.d.-process in a system, look for $\alpha \neq 0$; for what physics drives the non-linearity, look at $B$. $* * *$ 


\section{On D Being Proportional to [v+]}

The vacancy mechanism for diffusion sets the diffusivity roughly proportional to the (t)-ion vacancy concentration (for cation diffusion). In the 'standard' model, the $[\mathrm{v}+]$ concentration is whatever concentration that appears in the equilibrium constraint: " $n(v+) n(v-)=\underline{K}_{0}(T) . "$ With the addition of $(\mathrm{v}+)+(\mathrm{v}-) \longleftrightarrow(\mathrm{d})$ there is an ambiguity as to which $([\mathrm{v}+]$ or $[\hat{\mathrm{v}+}])$ concentration is to be used in a $\mathrm{D} \sim "[\mathrm{v}+]^{i}$ model.

In the most simplistic formalism, if the divacancies do not participate in the diffusion process (i.e., (v-)-sites are viewed as immobile trapsites) then $D \sim\left[v^{+}\right]$(the free $(t)$-ion vacancy concentration). If the $(v-)$-sites, were extremely mobile, a vacancy $\left(v^{+}\right)$, whether free or within a bound divacancy, should still participate in diffusion. Then one would expect $D \sim[\hat{\mathrm{v}}+]$ (the total $(+)$-ion vacancy concentration).. The 'standard' model of $\mathrm{D} \sim[\mathrm{vt}]$ (without specification of 'free' or 'total' vacancy concentration) relies on the fact that (for small vacancy concentrations) to double the number of vacancies is to (roughly) double the number of encounters a diffusing ion will have with vacancies, and thus the flux of diffusing ions will double. It is an equivalent level of formalism to use the fact that at a fixed concentration (again small; as used in the sense of the 'standard' model), to double the vacancy mobility roughly doubles the vacancy jumps/sec., which doubles. the number of encounters a diffusing ion will have with vacancies, and one could expect that the flux of the diffusing ion doubles. Thus:

$$
D(C) \sim \mu(v+) n(v+)
$$

where $\mu(s)$ denotes the mobility of the species 's'.' Usually the specific 
accounting of mobility is not done explicitly in $D$, but, realizing that the mobility of a free $(v+)$ and a $(v t)$ which is bound in a divacancy may be quite different, depending on the mobility of the $(v-)$ defect, an accounting of the mobility of the vacancy must be made in the attempt to answer whether $D \sim[v+]$ or $D \sim[\hat{v}+]$ should be used. Within the context of using mobilities, the answer is quite easy: ${ }^{32}$

$$
\mathrm{D} \sim(\mu(\mathrm{vt}) \mathrm{n}(\mathrm{vt})+\mu(\mathrm{d}) \mathrm{n}(\mathrm{d}))
$$

This is the generalization of the original two-fold choice of using [vt] or $[\hat{v}+]$. This formalism has the additional feature that the $n(s)$ term (for the species 's') scales roughly as (exp $\left.-G^{F} / k T\right)$, with $G^{F}$ the formation energy, and the $\mu(s)$ term scales roughly as $\left(\exp -G^{M} / k T\right)$, where $\mathrm{G}^{\mathrm{M}}$ is the migrational energy.

Whereas $\mu(v t)$ is a clear concept, the $(v t)$ being a simple defect (occupying one lattice site), the $\mu(d)$ is more complicated, since the divacancy is an 'extended' (over 2 lattice sites) defect (though not like the true extended defects:-dislocations, domain walls, etc.). As a bound entity, it has a characteristic binding energy, but that should primarily affect its tendency to disassociate, and not its mobility while in the bound state. 32 One thus may expect some kind of basic kinematic relationship between $\mu(d)$ and the mobility of its component parts: $\mu(v+), \mu(v-)$. Since the motion of a cluster-defect is limited by the mobility of the slowest species in cluster, this suggests that $\mu(d)$ can be taken as a reduced mobility: ${ }^{32}$

$$
\mu(\mathrm{d})^{-1}=\mu\left(v^{+}\right)^{-1}+\mu\left(v^{-}\right)^{-1}
$$

The concept of reduced mobility certainly: matches what is intuitively 
expected in the following limits:

(1). The Trapsite Limit: $\mu(d)=0$ irrespective of $\mu(v+)$ because an immobile ( $v-$ ) defect will tend to have the associated (vt) circulating around it, and as a trapped entity, the (v+) cannot participate in bulk ion diffusion

(2). The Unhampered Limit: $\mu(v+)=\infty$ gives $\mu(d)=\mu(v-)$. This is the degenerate case where the cluster mobility is exactly the mobility of its slowest member. There the ( $t+$ ) would circulate so fast around the (v-) that it can be near wherever the $\left(v^{-}\right)$is and thus the $(v+)$ does not change $\left(v^{-}\right)$mobility.

(3). The Equimotional Limit: $\mu(v+)=\mu(v-)$ gives $\mu(d)=\frac{1}{2} \mu(v+)$. Even if the $(v+)$ and $(v-)$ defects could move with equivalent ease, (indeed, some recent calculations indicate that $\mathrm{G}^{\mathrm{M}}(\mathrm{v}+)$ and $G^{M}(v-)$ in some ionic crystals may not be that disparate ${ }^{33}$ ) the fact that $\mu(\mathrm{d})$ is monotonic in each variable, $\mu(\mathrm{v}+), \mu(\mathrm{v}-)$; and that the $\mu(d)=\mu(v-)$ is reserved for the $\mu(v+)=\infty$ case, makes it not too surprising that $\mu(d)$ cannot exceed half of $\mu(v+)$ (given $\mu(v+) \geq \mu(v-))$.

The mobility is usually constituted from factors as the hopping frequency (which is where exp-GM/kT usually enters) and the hopping distance. In simple salts (like $\mathrm{NaCl}$ ) both the anion and cation lattices are structurally similar (same hopping distance and hopping geometry) and, since there is no correlation factor for vacancies, one can take:

$$
\frac{\mu(v+)}{v(v+)}=\frac{\mu(v-)}{v(v-)}
$$

i.e., the mobilities of ( $\left.v^{ \pm}\right)$are directly proportional to their actual 
hopping frequencies $v(v \pm)$, with the same proportlonality constant. This gives:

$$
\begin{aligned}
& v(d)=v(v+) v\left(v^{-}\right) /\left(v(v+)+v\left(v^{-}\right)\right) \\
& D \sim\left\{v\left(v^{+}\right) n\left(v^{+}\right)+v(v-) n\left(v^{-}\right)\right\}
\end{aligned}
$$

One can further write

$$
v(v \pm) \sim(v \pm) \exp -(G \pm) / k T
$$

where $(G \pm$ ) is the motional gibbs energy (and must be the same for a given ion or its ion-vacancy, since a vacancy hop is a host-ion hop in the opposite direction; i.e., $\left.(G+)=G^{M}\left(\mathrm{Na}^{+}\right),\left(G^{-}\right)=G^{M}\left(\mathrm{Cl}^{-}\right)\right)$. Similarly, the pre-exponential factors $(v \pm)$ should be the same as the hostion pre-exponential factors $\left((\nu+)=\nu_{0}\left(\mathrm{Na}^{+}\right),\left(\nu_{-}\right)=\nu_{0}\left(\mathrm{Cl} l^{-}\right)\right.$; where $\nu_{0}$ is some jump attempt frequency).

The usual model for diffusion is:

$$
D=\left(1 / \mathrm{N}_{0}\right) \gamma \mathrm{a}_{\mathrm{o}}^{2} \mathrm{f}
$$

where $\left(1 / \mathrm{N}_{0}\right)$ is a geometrical factor depending on lattice type and nearest neighbor number, ${ }^{34} a_{0}$ is a hopping length, $\gamma$ is an actual hopping frequency, and $f$ is a correlation factor. Specializing to a situation such as

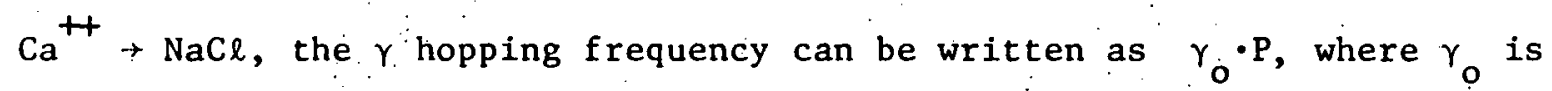

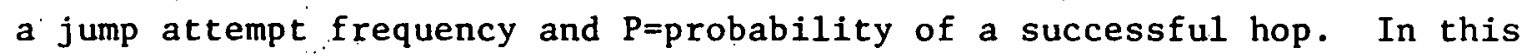
example, ' $P$ ' will have a term $\exp -\left(G^{M}\left(\mathrm{Ca}^{++}\right) / \mathrm{kT}\right)$, which is the probability that a random thermal fluctuation can induce an ion 'hop' over the potential barrier characterized by $\mathrm{G}^{\mathrm{M}}\left(\mathrm{Ca}^{++}\right)$. Since a $\mathrm{Ca}^{++}$impurity can significantly alter the local lattice near it, one does not expect $G^{M}\left(\mathrm{Ca}^{++}\right) \simeq G^{M}\left(\mathrm{Na}^{+}\right)$. Similarly, for this example, $\gamma_{0}\left(\mathrm{Ca}^{++}\right)$is not equal 
to $v_{0}\left(\mathrm{Na}^{+}\right)$, because the atoms are trapped in different local potentials. Another term in ' $P$ ' is the fractional-site-occupation by $(+)$-ion vacancies. Thus, for the simple vacancy mechanism model:

$$
D=\left(1 / N_{0}\right) \mathrm{fa}_{0}^{2} \mathrm{n}(\mathrm{v}+) \gamma_{0}\left(\mathrm{Ca}^{++}\right) \exp -\left(\mathrm{G}^{\mathrm{M}}\left(\mathrm{Ca}^{++}\right) / \mathrm{kT}\right)
$$

This derivation, although quite standard, stands contradistinct to the previously presented argument that (even in the simple vacancy mechanism with no divacancy contribution) one should have $D \sim \mu(v+) n(v t)$ and not just $D \sim n(v+)$ alone. The $D \sim n(v+)$ model has inherent in its derivation the factor $\exp -\left(G^{M}\left(C^{++}\right) / k T\right)$, while a model $D \sim, \mu(v+) n(v t)$ has the factor exp- $\left(\mathrm{G}^{\mathrm{M}}\left(\mathrm{Na}^{+}\right) / \mathrm{kT}\right)$, and one expects that this is a significant difference (i,e., $\mathrm{G}^{\mathrm{M}}\left(\mathrm{Ca}^{++}\right) \neq \mathrm{G}^{\mathrm{M}}\left(\mathrm{Na}^{+}\right)$). Turning now to the further model $D \sim\{v(v t) n(v+)+v(d) n(d)\}$, one cannot, ad hoc, scale away this disparity by setting:

$$
D: \frac{\{(v+) n(v+)+v(d) n(d)\}}{(v(v+)+v(d))}
$$

or by any similar expression which would introduce an ad hoc factor of inverse frequency. The reason is that if such a factor were introduced, D would no longer scale with mobility.

We offer one possible resolution of this paradox.

\section{The Hopping Frequency}

There are two frequencies in question here:

$$
v_{A}=\{v(v+) n(v+)+v(d) n(d)\}
$$

which is an effective frequency for encountering a (vt), and

$$
\nu_{B}=\dot{\gamma}_{0} \exp -G^{M}\left(\mathrm{Ca}^{++}\right) / \mathrm{kT}^{+}
$$


which is an (impurity) lon hopping frequency given that a vacancy is nearby. For simplicity, we first deal with the $\nu(d)=0$ case. The aforementioned "paradox" still exists since $v(v+) \sim \exp -G^{M}\left(\mathrm{Na}^{+}\right) / \mathrm{kT}$ and $\nu_{B}$ has a. $G^{M}\left(\mathrm{Ca}^{++}\right)$factor. The essence of the problem lies in the decision of what frequency really represents the "true" hopping frequency for use in D. $\nu_{B}$ cannot by itself because it assumes the continual presence of a $(v+) . \quad v_{A}$ cannot by itself because it bears no reference to the hopping atom. In some sense, the answer must lie in some explicit time-consideration. Although we do not want to introduce time explicitly to give a $D(t)$, we introduce time via the following equivalence. 35

A single atom hopping with a fixed frequency $v_{0}$ on an open lattice gives the same probability distribution as an atom hopping via the Poisson distribution:

$$
P(N, t)=\frac{1}{N !}\left(\nu_{0} t\right)^{N} \exp -\left(\nu_{0} t\right)
$$

where $P(N, t)=$ [probability of exactly $N$ hops in a time $t$ ]. In other words, the diffusion of a hopping atom governed by a fixed frequency $\nu_{0}$, and one governed by the decay law:

$$
P(N=0, t)=\exp -\left(\nu_{0} t\right)
$$

are identical ( $\mathrm{N}=0$ being analogous to remaining at a given site). This is because the average hopping frequency of this Poisson process is also $\nu_{0}$ and since both distributions have the same average hopping frequency, they will develop at the same rate (both, of course; are gaussians; they have now been demonstrated to be gaussians of equivalent width). For the Poisson process, since:

$$
\langle\bar{v}\rangle=\left\langle\frac{N}{t}=v_{0}\right.
$$


the average number of hops in a time ' $t$ ', $\langle N\rangle$, must then be given by $<N=v_{0} t$

Thus, $\nu_{B}$ is not only the (average) hopplng frequency given a vacancy nearby, but also it must represent, via

$$
P_{1}=1-\exp -\left(\nu_{B} t\right)
$$

the probability that an ion (given the vacancy) will hop into the vacant site. Turning to the $\nu_{A}$ equation $(v(d)=0$ limit $)$ :

$$
v_{A}=v(v+) n(v+)
$$

it is some kind of reduced (effective) frequency. A numerical example will help clarify an interpretation of $v_{A}$. Suppose $n(v+)=10^{-6}$ (1 p.p.m. vacancies) and $v(v t)=1$ jump/sec. The probability of any given lattice site having a vacancy is $10^{-6}$, and the $v(v+)$ is the visits/sec a vacancy can make, so the 1 visit/sec. is spread over $10^{6}$ possible sites, for a $\nu_{A}=10^{-6}$ visits/sec $=$ (average number of visits/sec.) to a given lattice site! Using this interpretation, if $v(v+)$ were a fixed dynamic frequency, then, in a time ' $t$ ', the average number of visits by any vacancy to a given lattice site is $\left(v_{A} t\right)$. One can now convert this to a Poisson process where:

$$
\mathrm{P}_{2}=1-\exp -\left(v_{A} t\right)
$$

is the probability that a given site will be visited (as a function of time by some vacancy). This Poisson process also retains $\left(\nu_{A} t\right)$, being the average number of visits in a time ' $t$ '.

The average length of a visit of a vacancy at a given lattice site relies solely on $v\left(v^{t}\right)$; and converting the hopping of the vacancy to a Poisson process too, one easily finds the average visitation time 
is $1 / v(v t)$. A given lattice site, as a function of time, now recelves $\left(\nu_{A} t\right)$ visits of duration $(1 / v(v+))$, so that $\left(v_{A} t / v(v+)\right)$ is the amount of time a given lattice site is occupied by a vacancy. The fraction of time that some vacancy occupies the lattice site is then $\left(v_{A} / \nu(v t)\right)=$ $\mathrm{n}(\mathrm{v}+)$, as it should be:

Returning to the atom motion, if at $t=0$ a vacancy hops near to an atom, this is labelled as 'visit-1' of average duration $\hat{T}=1 / v(v t)$. In this amount of time, with ' $P$ ' a probability:

$$
\begin{aligned}
& P(\text { atom hops })=1-\exp -\left(\nu_{B} \hat{T}\right) \\
& P(\text { no hop, visit }-1)=\exp -\left(\nu_{B} \hat{T}\right)
\end{aligned}
$$

One next allows $\mathrm{N}$ visits:

$$
P(\text { no hop in } N \text { successive visits })=\left[\exp -\left(\nu_{B} \hat{T}\right)\right]^{N}
$$

Easily then, the probability of at least one atomic hop in ' $N$ ' vacancy visits is :

$$
P(\text { one or more hops, } N \text { visits })=1-\left[\exp -\left(\nu_{B} \hat{T}\right)\right]^{N}
$$

The average time before $N$ visits to a site can accrue is $\nu_{A} t=N$; thus, one has:

$$
\begin{aligned}
P(\text { one or more hops }) & =1-\exp -\left(\nu_{B} \hat{\mathrm{T}}_{A} t\right) \\
& =1-\exp -\left[\left(\nu_{B} \nu_{A} / \nu(v t)\right) t\right] .
\end{aligned}
$$

Putting in $\nu_{B}$ and $\nu_{A}$ one gets:

$$
P(\text { one or more hops })=1-\exp -\left[\left(\gamma_{0} \exp -G^{M}\left(C a^{++}\right) / k T\right)(n(v+) t)\right]
$$

This now is treated as a compound Poisson process with characteristic frequency:

$$
v_{C}=\gamma_{o} n(v+) \exp \div\left[G^{M}\left(\mathrm{Ca}^{++}\right) / \mathrm{kT}\right]
$$


This rederives the standard model, but in so doing, it gives a framework suitable for generalization to the $v(d) \neq 0$ case.

$$
\text { * * * }
$$

Turning now to the full $v_{A}$ expression, it still represents the frequency of visits to a given lattice site by a ( $v+$ ) (which may now be free or bound to $(v-))$. A given site still receives an average of $\left(v_{A} t\right)$ visits in a time ' $t$ '. The duration of a visit, whether the $(v t)$ is bound or free, is: $\hat{\mathrm{T}}($ duration $)=1 / \mathrm{v}(\mathrm{v}+)$. This is a delicate point, a weighted average:

$$
\hat{\mathrm{T}} \stackrel{?}{=} \frac{[\mathrm{n}(\mathrm{v} t) / v(v+)]+[\mathrm{n}(\mathrm{d}) / v(\mathrm{~d})]}{[\mathrm{n}(\mathrm{vt})+\mathrm{n}(\mathrm{d})]}
$$

diverges as $v(d) \rightarrow 0$, which certainly is incorrect. To set the duration ' $\hat{T}$ ' as $\hat{T}=1 / \nu(v+)$ means a given site will have a vacancy on it just so long whether it is bound to a divacancy or not: The only difference then is: "If $(v+)$ is bound to a divacancy, the (vt) hops to circulate about the $\left(v^{-}\right)$, and, to lst order, this will not affect the lifetime of a $(v+)$ on a given site. This alșo gives, when $\nu(d) \rightarrow 0$, no divacancy contribution to either $\hat{T}$ or $\left(v_{A} t\right)$, as it should be. Finally, as before,

$$
\mathrm{P} \text { (one or more atom hops) }=1-\exp -\left(\nu_{B} \hat{\nu}^{\hat{T} t}\right)
$$

which gives an effective frequency of $\nu_{C}=\nu_{B} \nu_{A} \hat{T}$ :

$$
\begin{aligned}
v_{C} & =\gamma_{0}\left(\exp -G^{M}\left(\mathrm{Ca}^{+}\right) / k T\right)(n(v+)+n(d) \nu(d) / \nu(v+)) \\
& =\gamma_{0}\left(\exp -G^{M}\left(\mathrm{Ca}^{++}\right) / k T\right)(n(v+)+Q(T) n(d)) \\
Q(T) & =\frac{\exp -\left[G^{M}\left(\mathrm{Cl}^{-}\right)-G^{M}(N a+)\right] / k T}{\exp -G^{M}\left(\mathrm{Na}^{+}\right) / k T+\exp -G^{M}\left(C e^{-}\right) / k T}
\end{aligned}
$$


The derived resolution is that while. $D \sim\left(\sum_{\mu(i) n(i))}\right.$ is true as a proportionality, it is not truly a scaling. To have $D$ scale with mobility is incorrect; or alternately, to double the hopping frequency for ( $v+$ ) is to halve the visitation time, giving no net gain.

\section{Summary of $D(C)$ Models}

In this section, the various models presented earlier are collected and summarized (using the standard notation of $[\hat{s}]=$ (total concentration) and $[s]=$ (free unbound concentration). $)$. Model-specific limiting concentration diffusivities $\left(D_{1}, D_{2}, \ldots\right)$ are introduced which represent explicit functions of other (microscopic) variables, reserving $D_{0}$ for the model unspecific limiting concentration diffusivity. For some cases $\mathrm{D}_{0} \longleftrightarrow \mathrm{D}_{1}$ and for others, $\mathrm{D}_{0} \longleftrightarrow \mathrm{D}_{2}$.

(1). No impurities, no divacancy thermodynamics, equilibration with extended defects may be present or not.

$$
D \sim \hat{n}\left(v^{+}\right)
$$

Since there is no binding between $(v+),(v-)$, there are no "trapped" $(v+)$-sites, so ' $n(v+)$ ' must refer to the total fractional-site-occupation by $(v t)$ vacancies. With $\hat{n}(v t)=\hat{n}(v-) \equiv \hat{n}_{0}(v t)=\hat{n}_{0}(v-) \equiv \hat{n}_{0}$ :

$$
\mathrm{D}_{1}=(1 / \mathrm{N}) \mathrm{fa}_{\mathrm{o}}{ }^{2} \dot{\gamma}_{\mathrm{o}} \hat{\mathrm{n}}_{\mathrm{o}}\left(\exp -\mathrm{G}^{\mathrm{M}}\left(\mathrm{Ca}^{++}\right) / \mathrm{kT}\right)
$$

(2). Aliovalent impurities, no divacancy thermodynamics, no equilibrations with extended defects:

$$
D \sim \hat{n}(v t)
$$




$$
\begin{aligned}
& {[\hat{\mathrm{v}}+]=\left[\hat{\mathrm{v}}_{0}\right]+\mathrm{mC}, \quad \mathrm{p} \equiv\left[\mathrm{v}_{0}\right] /\left[\mathrm{d}_{0}\right]} \\
& \hat{\mathrm{K}} \equiv \mathrm{mC} /\left[\hat{\mathrm{v}}_{0}\right] \quad \mathrm{K} \equiv \mathrm{mC} /\left[\mathrm{v}_{0}\right] \\
& \hat{\mathrm{K}}=\mathrm{K}\left[\mathrm{v}_{0}\right] /\left(\left[\mathrm{v}_{0}\right]+\left[\mathrm{d}_{0}\right]\right)=K(\mathrm{p} /(1+\mathrm{p})) \\
& \therefore(\mathrm{C})=\mathrm{D}_{1}[\hat{\mathrm{v}}+] /\left[\hat{\mathrm{v}}_{0}\right]=\mathrm{D}_{1}(1+\hat{\mathrm{K}})=\mathrm{D}_{1}(1+(\mathrm{p} /(1+\mathrm{p})) \mathrm{K})
\end{aligned}
$$

(3): Aliovalent impurities, no divacancy thermodynamics, equilibration with extended defects added:

$$
\begin{aligned}
D & \sim \hat{n}(v+) \\
\therefore(C) & =D_{1} \cdot\left[(\hat{K} / 2)+\left[1+(\hat{K} / 2)^{2}\right]^{1 / 2}\right] \\
& \simeq D_{1}\left[1+(\hat{K} / 2)+\left(\hat{K}^{2} / 8\right)+\ldots\right]
\end{aligned}
$$

The ratio $r=\left(\operatorname{coefficient} \hat{\mathrm{K}}^{2}\right.$ term) $/(\operatorname{coefficient} \hat{\mathrm{K}} \text { term })^{2}=.5$ for this model:

(4). No impurities, divacancy thermodynamics added; equilibration with extended defects may be present or not:

$$
\begin{aligned}
& D \sim(n(v+)+Q(T) n(d)) \sim \hat{n}_{0}\left[n_{0}(v+) / \hat{n}_{0}(v+)\right] \cdot\left[1+Q(T)\left[d_{0}\right] /\left[v_{0}\right]\right] \\
& 1 \geq Q(T) \geq 0, \quad p=\left[v_{0}\right] /\left[d_{0}\right] \\
& Q\left(I^{\prime}\right)=\frac{\exp -\left[G^{M}\left(C \ell^{-}\right)-G^{M}\left(N^{+}\right)\right] / k T}{\left(\exp -G^{M}\left(N a^{+}\right) / k T\right)+\left(\exp -G^{M}\left(C l^{-}\right) / k T\right)} \\
& D_{2}=D_{1}(p /(1+p))\left(1+Q(T) p^{-1}\right)=D_{1}(p+Q(T)) /(1+p) \leq D_{1}
\end{aligned}
$$

(5). Aliovalent ions, divacancy thermodynamics added, no equilibration with extended defects:

$$
\begin{aligned}
& D=D_{1}(p /(1+p))([v+]+Q(T)[d]) /\left[v_{0}\right] \\
& {[v+] /\left[v_{0}\right]=\left[(1+p)+(K+p)^{2} / 4\right]^{1 / 2}+\frac{1}{2}(K-p)}
\end{aligned}
$$




$$
[\mathrm{d}] /\left[\mathrm{v}_{\mathrm{o}}\right]=1+\mathrm{p}{ }^{-1}+\mathrm{K}-[\mathrm{v}+] /\left[\mathrm{v}_{\mathrm{o}}\right]
$$

$p>>1$ (few divacancy limit):

$$
\begin{aligned}
D(C) & \simeq D_{1}(p /(1+p))\left[(1+K)\left(1+Q p^{-1}\right)-(K / p)+\left[K(2+K)(1-Q) / p^{2}\right] \ldots\right] \\
& \simeq D_{2}[(1+K)-[K /(p+Q)]-[K(2+K)(1-Q) / p(p+Q)] \ldots]
\end{aligned}
$$

$\mathrm{p}<<$ (many divacancy limit):

$$
\begin{aligned}
D(C) \simeq & D_{1}(p /(1+p))\left[\left(1+Q p^{-1}+[(1+Q) K / 2]+\right.\right. \\
& \left.(1-Q)\left[\left(K^{2} / 8\right)+[1-(K / 4) \cdots(p / 2)](K p / 4)\right] \ldots\right] \\
\simeq & D_{2}\left[1+\frac{1}{2} K([(Q+1) /(Q+p)]-[(1-Q) p / 2(Q+p)] \ldots)+(1-(1-Q) p / 2(Q+p) \ldots) K^{2} / 8 \ldots\right]
\end{aligned}
$$

(6). Aliovalent ions, divacancy thermodynamics, and equilibration with extended defects both added:

$$
\begin{aligned}
D(C) & \simeq D_{1}(p /(1+p))\left[\left(1+Q p^{-1}\right)+\frac{1}{2} K+\left(K^{2} / 8\right)(1-(p+2 Q) /(p+1)(p+2)) \ldots\right] \\
& \simeq D_{2}\left[1+(p /(Q+p))\left[\frac{1}{2} K+\left(K^{2} / 8\right)(1-(p+2 Q) /(p+1)(p+2))\right] \ldots\right]
\end{aligned}
$$

The ratio $\mathbf{r}=.5(1-(p+2 Q) /(p+1)(p+2))<.5$ for this model.

These models are all characterized by one of two distinct zeroimpurity diffusivities, $D_{1}$ and $D_{2} ;$ where

$$
\mathrm{D}_{2}=[(\mathrm{p}+\mathrm{Q}) /(1+\mathrm{p})] \mathrm{D}_{1}
$$

can vary from being almost as large as $D_{1}$ to a much smaller quantity if both $\mathrm{p}$ and $\mathrm{Q}$ are small (indicitive of many immobile divacancies). The term proportional to $\mathrm{K}=\mathrm{mC} /\left[\mathrm{v}_{\mathrm{o}}\right]$ in a $\mathrm{D}(\mathrm{C})$ problem can be small (as $\mathrm{p} /(1+\mathrm{p})$ in mode1-2, if $\mathrm{p}$ is small) to order unity (as in mode1-5 if $\mathrm{p}$ is large; or in model-6 if both $Q$ and. $p$ are small), to a number significantly 
larger than unity (as in model-5 if $p$ and $Q$ are both sma11): The difficulty is that since only one given situation is present in a given crystal with a given diffusant at a given temperature, the differences between these "large" and "small" effects cannot be directly measured except possibly in a comparitive study between different crystals under controlled modified conditions.

In any one system, the major distinction among these models that can be directly 'measured', which could qualitatively indicate which model is 'more correct' for the system, is the parameter ' $\mathrm{r}$ ':

$$
\begin{array}{ll}
r=\left(\operatorname{coeff.} \mathrm{K}^{2}\right) /(\operatorname{coeff} . \mathrm{K})^{2} & \text { General Definition } \\
\mathbf{r}=0 & \text { model-2; model-5 if } \mathrm{p}>>1 \\
\mathrm{r}=.5 & \operatorname{mode1-3}(\text { 'standard' model) } \\
\mathrm{r}=.5(\mathrm{Q}+\mathrm{p})^{2} /(\mathrm{Q}+1)^{2}<.5 & \operatorname{mode1}-5 \text { if } \mathrm{p}<<1 \\
\mathrm{r}=.5(1-(\mathrm{p}+2 \mathrm{Q}) /(\mathrm{p}+1)(\mathrm{p}+2)) & \operatorname{model}-6
\end{array}
$$

For metal-ion diffusion in $\mathrm{NaCl}$, if one has $\mathrm{G}^{\mathrm{M}}\left(\mathrm{C} \ell^{-}\right)>>\mathrm{G}^{\mathrm{M}}\left(\mathrm{Na}^{+}\right)$, then model-5 will give $r \simeq 0$ in the $p<<1$ limit as well as in the $p>>1$ limit. With this added assumption, one gets:

$$
\begin{aligned}
\mathbf{r} & \simeq 0 \\
\mathbf{r} & =.5 \\
.4 \leq \mathbf{r} & <.5
\end{aligned}
$$

Finally, a value of $r>\frac{1}{2}$. would indicate a $D(C)$ process which is quite unlike any in the set considered here.

$$
* * *
$$

In this chapter, we have reviewed the 'standard' $D(C)$ model for aliovalent ion diffusion in ionic crystals, as well as having pointed out 
some possible 'weakness' in this 'standard' formation. Extending

this basic model to handle the additional process of divacancy association, the $D(C)$ process still fundamentally remains; and writing $D(C)$ as a power series in $K=m C /\left[v_{0}\right]$, the coefficients of each $k^{n}$-term (for different $n$ ) vary from model to model. The parameter ' $r$ ' may begin to differentiate among these models, but it would require $D(C)$ to be taken out to the $\mathrm{K}^{2}$-term.

Also, these models are incomplete in two very important aspects:

(1). We have nowhere considered the possibility of (even short-term) association of aliovalent impurities and vacancies (as $\mathrm{Ca}^{++}$ -(vi) complexes); such a process would necessitate coupled diffusion equations, as well as the separation of the aliovalent ion concentration (at each point in space) into two components $c$ (free) and $c$ (bound), and thus it is a degree greater complexity than a simple $D(C)$ process (2). We have also nowhere allowed the possibility that the vacancy equilibria that we have assumed might not truly be in equilibrium in a given experimental situation. This could make $n(v t)$ a function of time and/or space; and again one would need to use a coupled diffusion equation formulation requiring at least an extra diffusion equation for the $n(v t)$.

In the next chapter the formal non-1inear anaiysis is introduced and the $D(C)=D_{0}(1+\alpha C)$ problem is considered. It will use an extension of methodology presented in Chapter 2, and, even though it will not be able to differentiate among the different models presented here, the parameter $\alpha$ still is the first measure of non-linearity present in any given system. 
CHAPTER 4

NON-LINEAR ANALYSES AND THE $D=D_{0}(1+\alpha C)$ PROBLEM

\section{The Lore of Concentration-Dependent Diffusion}

Some of the earliest work was due to Boltzmann 36 and extended to various situations by Matano. ${ }^{37}$ With $D=D_{0}(1+\gamma f(C))$ and $z=x /\left[4 D_{0} t\right]^{1 / 2}$ the equation.

$$
\frac{\partial C}{\partial t}=D_{0} \frac{\partial}{\partial x}(1+\gamma f(C)) \frac{\partial C}{\partial x}
$$

transforms to: $38 \cdot \frac{\partial}{\partial z}(1+\gamma E(C)) \frac{\partial C}{\partial z}+2 z \frac{\partial C}{\partial z}=4 t \frac{\partial C}{\partial t}$

If the boundary conditions are one of the following two special cases:

1. $\left(C=C_{0}\right)$ for $(x<0, t=0)$; and $(C=0)$ for $(x>0, t=0) ; x=(-\infty,+\infty)$

2. $\left(C=C_{0}\right)$ for $(x=0, t \geq 0)$; and $(C=0)$ for $(x>0, t=0) ; x=(0, \infty)$

then $\frac{\partial}{\partial t} C(z, t):=0$, and one gets the following non-linear ordinary differential equation from Eq. (4.02):

$$
\begin{gathered}
(1+\gamma f(C))=-2\left(\frac{\partial z(C)}{\partial C}\right) \int_{C}^{C} z\left(C^{\prime}\right) d C^{\prime} \\
\vdots \quad \therefore \quad . \quad C_{0}
\end{gathered}
$$

where since ' $C$ ' is a function of ' $z$ ' only, it is viewed as defining an implicit function $z(C)$. J. R. Philip ${ }^{39}$ has found $C(z)$ as the inverse of some $z(C)$ for a specialized class of $D(C)$ functions. Pelton and Etsel1 40 have considered these two cases for $D \sim C$, developing $C(z)$ as a converging series in ' $z$ '. M. Ghezzo ${ }^{41}$ has demonstrated that one can get an ordinary differential equation given a situation where ' $D$ ' can be written as $D(\xi)$, 
where $\xi=\mathrm{f}(\mathrm{z}) / \mathrm{f}(\mathrm{z}=0)$ with $\mathrm{C}(\mathrm{x}, \mathrm{t}=0)=\mathrm{N} \delta(\mathrm{x})$, and that $\int \mathrm{C}(\mathrm{x}, \mathrm{t}) \mathrm{dx}$. can be made independent of time for this situation. Most of the work in the field has centered about those specific situations in which $c(z, t)$ can be made into a $C(z)$ problem, thereby reducing the partial differential equation to an ordinary one; as is true for every article cited above:. Unfortunately, the transition to a 'thin-source' type boundary condition (where both $D=D(C)$ and $\int C(x, t) d x$ being independent of time need be satisfied) may necessitate use of both dynamical variables: $(z, t)$.

One construction that began to tackle the 'thin-source' situation, again by M. Ghezzo, ${ }^{42}$ when $D \sim C^{n}$, for the boundary condition:

$$
(C(x, t) \rightarrow \infty) \text { for }(x=0, t=0) \text {; and }(C(x, t)=0) \text { for }(x>0, t=0) \text { and for }(x \rightarrow \infty, t \geq 0)
$$

which preserves $\int c(x, t) d x$ being independent of time, results in:

$$
\begin{aligned}
& C(x, t)=\left\{\begin{array}{cc}
(A t)^{-1 /(n+2)}\left[B-.5 n x^{2}(A t)^{-2 /(n+2)}\right]^{1 / n}, & 0 \leq x \leq \phi(t) \\
0, \therefore x>\phi(t) & \ddots
\end{array}\right. \\
& \phi(t)=(A t)^{1 /(n+2)} \cdot(2 B / n)^{1 / 2}
\end{aligned}
$$

where ' $A$ ' and ' $B$ ' are non-arbitrary constants. The most serious shortcoming of this solution is that at $x=\phi(t)$, for all time, there is a jump in the slope of $C(x, t) ; i . e .$, this is a non-analytic solution with a propagating discontinuity. Beniere et al. ${ }^{43}$ have taken various $D(C)$ functions derived from the physics of mass action (similar to the presentation of Chapter 3 ), along with $C(x, t=0) \simeq 1 \delta(x)$, and, using finitedifference approximations to differential equations and numerical methods, they have obtained $C(x, t)$ profiles (for various choices of parameters) that seem to match actual diffusion data in some ionic crystals. 
M. D. Feft ${ }^{44}$ et al. were some of the first to work on an analytic solution applicable to a 'thin-source' boundary condition for the $D(C)=$ $\mathrm{D}_{0}(1+\alpha C)$ problem. Starting from a Fourier transform methodology, an iterative method was constructed which expressed $c(x, t)$ as:

$$
C(x, t)=f(t) \exp -\left(f_{0}(x, t)+\alpha f_{1}(x, t)+\alpha^{2} f_{2}(x, t) \ldots\right)
$$

and $C(x, t)$ was calculated explicitly to order- $\alpha$ (in the exponent). J. L. Mitchel1 $1^{44,45}$ et al. extended the analysis to order- $\alpha^{2}$ (in the exponent). The major difficulty with this method is that the approximations which are used to generate an answer change the problem being solved from one involving a differential equation with two dynamical variables: $(z, t)$, into one involving only a single dynamical variable, $z$, and time is treated as a parameter, much like $D_{0}$ and $\alpha$. The other difficulty is that, as an iterative method, each iteration is a "step", and the procedure requires forced renormalization to conserve $\int c(x, t) d x$ at each step since it does not intrinsically conserve total amount.

In general, a full physical solution to the $D(C)=D_{0}(1+\alpha C)$ problem (or any $D(C)$ problem), given a 'thin-source' boundary condition, should meet, if possible, the following requirements:

(1). If $C\left(\dot{x}, t_{R}\right)$ is an "initial state," it must be allowed to be an arbitrary integrable function of $x$

(2). $C\left(x, t>t_{R}\right)$ must be analytic in both variables: $(x, t)$ (3). The total amount $\int C(x, t) d x=N$ must be independent of time. Our aim, in the succeeding sections, is to construct such a solution for the $D(C)=D_{0}(1+\alpha C)$ problem. 


\section{On Scaling}

As seen earlier, various physical models give rise to different $D(C)$ functionals, and writing $D(C)$ as a small power series for small $C$ :

$$
D(C)=D_{0}\left(1+a K+b K^{2}+\ldots\right), \quad k=m C /\left[v_{0}\right]
$$

the $a, b .$. are numerical (pure number) parameters which depend on the various equilibrium constraints on vacancies, and $D_{0}$ is the limiting concentration diffusivity. A set of experiments which measure the profiles $\tilde{C}\left(x, t_{1}\right)$ for various diffusion times, $t_{i}$, has a normalization for each profile:

$$
\int \tilde{C}\left(x, t_{i}\right) d x=N(i)
$$

But, whereas if $\tilde{C}_{0}(x, t)$ solves the linear equation, so will $\lambda \tilde{C}_{0}(x, t)$, this is not true for any non-linear c.d.d. equation. Specifically, if $\tilde{C}_{0}(x, t)$ solves the non-linear equation with:

$$
\int \tilde{C}_{0}(x, t) d x=N_{0} ; \quad D(C)=D_{0}\left(1+a \tilde{K}+b \tilde{K}^{2} \ldots\right), \quad \tilde{K}^{2} \cdot \tilde{C}_{0} /\left[v_{0}\right]
$$

then the normed function $C_{0}(x, t)=\tilde{C}_{0}(x, t) / N_{0}$ solves an associated equation:

$$
\int C_{0}(x, t) d x=\hat{1},: D(C)=D_{0}\left(1+a\left(N_{0} K\right)+\ldots\right), \quad k=m C_{0} /\left[v_{0}\right] .
$$

In general, when attempting to correlate a set of data $\dot{c}\left(x, t_{i}\right)$, each with a total amount $N(i)$, one first creates normed profiles $c\left(x, t_{i}\right)=$ $\dot{C}\left(x, t_{i}\right) / N(i)$, and (the above argument shows that) each distinct $N(i)$ value then corresponds to a different $D(C)$ function (with the same $D_{0}$, but different coefficients for each power of C). Thus, a correlation should be performed using profiles with similar values of $N(i)$, and defining 
$\bar{N} \equiv$ (average of $N(1), N(2) \ldots$ ) then:

$$
\begin{aligned}
& \int C\left(x, t_{1}\right)=\hat{1}, \quad D(C)=D_{0}\left(1+\alpha C+B C^{2}+\ldots\right) \\
& \alpha=a\left(\bar{N} \mathrm{~m} /\left[v_{0}\right]\right), \quad \beta=b\left(\bar{N} m /\left[v_{0}\right]\right)^{2}
\end{aligned}
$$

and the associated physical parameters $\alpha_{0}=\alpha / \overline{\mathrm{N}}, \beta_{0}=\beta / \overline{\mathrm{N}}^{2}$ are ones which are independent of total impurity amount. We will, for the remainder of this thesis, use these definitions of $(\alpha, \beta, \ldots)$ and $\left(\alpha_{0}, \beta_{0}, \ldots\right)$ with $\left(D_{0}, a, b \ldots\right)$ as specified by the model of choice: (as given in Chapter 3 ).

\section{Notation and Sum Permutations}

We will often use arrays of functions as: $s_{m ; p}$, where both the array name (here.'S') is allowed to be compound (as, for example S, SA, SA2, S3A are all allowed distinct array names) as well as the subscript label. To avoid subscripts of subscripts, we will consistently use forms as $\left(m 1, m 2, m 3, \ldots p 1, p 2, p^{2} \ldots\right)$ instead of $\left(m_{1}, m_{2}, m_{3}, \ldots p_{1}, p_{2}, p_{3} \ldots\right)$. In the rare instances where the equivalent of $\left(m_{1}, m_{2}, \ldots m_{p}\right)$ is required we will use the notation $\left(\mathrm{m} 1, \mathrm{~m} 2, \ldots \mathrm{m}^{*} \mathrm{p}\right)$. An alternative to using subscripts (and superscripts) as indices of arrays, is a notation using parenthesis: $\mathrm{TA}(p, q)$ and $\mathrm{UA}_{p, q}$ both refer to the $(p, q)^{\text {th }}$-element of their respective arrays. Order is important: $\mathrm{TA}(\mathrm{p}, \mathrm{q}) \neq \mathrm{TA}(\mathrm{q}, \mathrm{p})$. If an array is defined in the format $T A(p, q)$, we will not suddenly use 'TA' $q^{\prime}$ '. Only through a formal equivalence as:

$$
\text { Define: } \mathrm{H}_{2 \mathrm{p}}(0) \equiv \mathrm{H}[2 \mathrm{p}]
$$

will such changes be made. Given an un-superscripted variable $\Gamma_{0}, \Gamma_{0}^{m}$ 
will represent its $\mathrm{m}^{\text {th }}$-power. For a superscripted variable $\mathrm{g}^{\mathrm{n}}$, its $\mathrm{m}^{\text {th }}$ power will be denoted $\left(\mathrm{g}^{\mathrm{n}}\right)^{\mathrm{m}}$, but attempts will be made to avoid such situations. No variable names, arrays, or labels shall begin with a number; thus $' 2 \mathrm{~N} 1$ ' $=\mathrm{N} 1+\mathrm{N} 1$.

Extensive use will be made of Kronecker's Deltas. They will be distinguished from (Dirac) $\delta$-functions by the presence of a symbol $(>, \geq,=, \leq,<)$. within the argument. Thus:

$$
\begin{aligned}
& \delta(a-b) \quad \text { is a Dirac } \delta \text {-function } \\
& \delta(a-b=0) \text { is a Kronecker's Delta. }
\end{aligned}
$$

The value assigned to a Kronecker's Delta is the following:

$$
\delta\left(\text { Kronecker's=Delta) }= \begin{cases}1 & \text { if the statement in the parenthesis is TRUE } \\ 0 & \text { if the statement in the parenthesis is FALSE }\end{cases}\right.
$$

Occasionally two labels may have the same range, as:

$$
\delta(N \geq m \geq 0) \quad \delta(N \geq n \geq 0)
$$

This we may combine into the form:

$$
\delta(\mathrm{N} \geq(\mathrm{m}, \mathrm{n}) \geq 0)
$$

and the logic structure is not altered: $(\delta(N \geq(m, n) \geq 0)=0$ if $m=N, n=N+1)$. This Kronecker's Delta formalism now can completely replace limits on sums. Whereas for the situation:

$$
\sum_{m=0}^{N} A_{m} t^{m}=\sum \delta(N \geq m>0) A_{m} t^{m}
$$

this Kronecker's Delta formalism may not be necessary, it is a definite advantage in a case like:

$$
\sum \delta(\min [p+q, m+n] \geq s \geq \max [p-n, q-m]) A_{s} t^{s}
$$


where $\max [a 1, a 2, \ldots]$ is the maximum of the set of arguments $(a 1, a 2 \ldots)$ and $\min [a 1, a 2, \ldots]$ Is likewise, the minimum of the set.

$$
* * *
$$

The next cases detail some important sum permutations that will be used in the next chapters.

CASE 1: Reverse Order Summing:

$$
\sum_{m=0}^{p} k(m, n)=\sum_{(p-m)=0}^{(p-m)=p} k(p-m, n)=\sum_{m=0}^{p} K(p-m, n)
$$

CASE $2: \therefore$ Sum Permutation:

$$
\begin{aligned}
f(t) & =\sum_{m=0}^{p} \sum_{n=0}^{m} K(m, n) \cdot t^{n}=\sum_{m=0}^{p} \sum_{n=0}^{p} \delta(m \geq n) k(m, n) t^{n} \\
& =\sum_{n=0}^{p} t^{n} \cdot \sum_{m=0}^{p} \delta(m>n) K(m, n)=\sum_{n=0}^{p} t^{n} \sum_{m=n}^{p} k(m, n)(4.15)
\end{aligned}
$$

CASE 3: Basic Additive Combination:

$$
f(t)=\sum_{m 1=0}^{\infty} \sum_{m 2=0}^{\infty} K(m 1, m 2) t^{(m 1+m 2)}
$$

Define $m \equiv m 1+m 2$ and replace ' $m 2$ ' by $m 2=m-m 1$ :

$$
f(t)=\sum_{m, m l} \delta(\infty \geq m l \geq 0) \delta(\infty \geq m-m l>0) K(m l, m-m l) t^{m}
$$

But $\delta(\infty>m-m l \geq 0)=\delta(\infty \geq m \geq m 1)=\delta(\infty \geq m \geq 0) \delta(m \geq m l)$ and

$$
\begin{aligned}
& \delta(\infty \geq m 1 \geq 0) \delta(m \geq m 1)=\delta(m>m 1 \geq 0) \quad \text { gives: } \\
& \begin{aligned}
\mathrm{f}(\mathrm{t}) & =\sum_{\because \mathrm{m}, \mathrm{ml}} \delta(\infty \geq \mathrm{m}>0) \delta(\mathrm{m}>\mathrm{ml} \geq 0) \mathrm{K}(\mathrm{ml}, \mathrm{m}-\mathrm{ml}) \mathrm{t}^{\mathrm{m}}
\end{aligned} \\
& =\sum_{m=0}^{\infty} t^{m} \sum_{m 1=0}^{m} K(m 1, m-m l)
\end{aligned}
$$


CASE 4: Additive Combination:

$$
\begin{aligned}
f(t)= & \sum_{m 1=0}^{N 1} \sum_{m 2=0}^{N 2} K(m 1, m 2) t^{(m 1+m 2)}= \\
& \sum_{m 1=0}^{\infty} \sum_{m 2=0}^{\infty} \delta(N 1>m 1) \delta(N 2>m 2) K(m 1, m 2) t^{(m 1+m 2)} .
\end{aligned}
$$

Define $\mathrm{m} \equiv \mathrm{m} 1+\mathrm{m} 2$, and replacing ' $\mathrm{m} 2$ ' by $\mathrm{m} 2=\mathrm{m}-\mathrm{ml}$ :

$$
f(t)=\sum_{m=0}^{\infty} \sum_{m 1=0}^{m} \delta(N 1>m 1) \delta(N 2 \geq m-m 1) k(m l, m-m 1) t^{m} .
$$

Since $\delta(\mathrm{N} 1 \geq \mathrm{m} 1) \quad \delta(\mathrm{N} 2 \geq \mathrm{m}-\mathrm{m} 1)=\delta(\mathrm{N} 1 \geq \mathrm{m} 1) \quad \delta(\mathrm{N} 2+\mathrm{m} 1 \geq \mathrm{m})=$

$\delta(\mathrm{N} 1 \geq \mathrm{m} 1) \quad \delta(\mathrm{N} 1+\mathrm{N} 2 \geq \mathrm{m}) \quad \delta(\mathrm{N} 2+\mathrm{ml} \geq \mathrm{m})=\delta(\mathrm{N} 1+\mathrm{N} 2 \geq \mathrm{m}) \quad \delta(\mathrm{m} 1 \geq \mathrm{m}-\mathrm{N} 2) \quad \delta(\mathrm{N} 1 \geq \mathrm{m} 1)=$ $\delta(\mathrm{N} 1+\mathrm{N} 2>\mathrm{m}) \quad \delta(\mathrm{N} 1>\mathrm{ml}>\mathrm{m}-\mathrm{N} 2)$, it gives:

$$
\begin{aligned}
f(t)= & \sum_{m=0}^{\infty} \delta(\mathrm{N} 1+\mathrm{N} 2 \geq \mathrm{m}) \sum_{\mathrm{m} 1=0}^{\mathrm{m}} \delta(\mathrm{N} 1 \geq \mathrm{m} 1 \geq \mathrm{m}-\mathrm{N} 2) \mathrm{K}(\mathrm{m} 1, \mathrm{~m}-\mathrm{m} 1) \mathrm{t}^{\mathrm{m}} \\
\therefore \quad & \sum_{\mathrm{m}=0}^{\infty} \delta(\mathrm{N} 1+\mathrm{N} 2>\mathrm{m}) \mathrm{t}^{\mathrm{m}} \sum_{(\mathrm{m} 1)} \delta(\mathrm{m}>\mathrm{m} 1>0) \delta(\mathrm{N} 1 \geq \mathrm{m} 1 \geq \mathrm{m}-\mathrm{N} 2) \mathrm{K}(\mathrm{m} 1, \mathrm{~m}-\mathrm{m} 1)= \\
\therefore \quad & \sum_{\mathrm{m}=0}^{\mathrm{N} 1+\mathrm{N} 2} \mathrm{t}^{\mathrm{m}} \sum_{(\mathrm{m} 1)} \delta(\mathrm{min}[\mathrm{m}, \mathrm{N} 1] \geq \mathrm{m} 1>\max [0, \mathrm{~m}-\mathrm{N} 2]) \mathrm{K}(\mathrm{m} 1, \mathrm{~m}-\mathrm{m} 1)
\end{aligned}
$$

\section{CASE 5: Alternate Additive Combination:}

$$
f(t)=\sum_{m l=0}^{N 1} r^{m l} \sum_{m 2=0}^{N 2} K(m 1, m 2) t^{(m 1+m 2)}
$$

Defining $m \equiv m 1+m 2$ and replacing ' $m 2$ ' with $m 2=m-m 1$ :

$$
\begin{aligned}
f(t)= & \sum_{m 1=0}^{N 1} r^{m 1} \sum_{(m)} \delta(N 2 \geq m-m l \geq 0) k(m l, m-m 1) t^{m}= \\
\because \quad & \sum_{m 1=0}^{N 1} r^{m 1} \sum_{m=m 1}^{N 2+m 1} K(m 1, m-m l) t^{m}
\end{aligned}
$$




\section{The Dynamica1 Variables $(z, t)$ and the Parameter $\mathrm{D}$}

As derived earlier, ${ }^{38}$ a transformation of $(x, t)$ into $\left(z_{0}, t\right)$ with $z_{0}^{2}=x^{2} / 4 D_{0} t$ changes

$$
\frac{\partial C}{\partial t}=D_{0} \frac{\partial}{\partial x}(1+\gamma f(C)) \frac{\partial C}{\partial x}
$$

into

$$
\text { 4t } \frac{\partial C}{\partial t}=\frac{\partial}{\partial z}(1+\gamma f(C)) \frac{\partial C}{\partial z}+2 z_{o} \frac{\partial C}{\partial z}
$$

Consider an arbitrary linear transformation on the time-variable:

$\Gamma=a\left(t+t_{0}\right)$ with a corresponding change to $z^{2}=z_{0}^{2}\left(t / t+t_{0}\right)=x^{2} / 4 D_{0}\left(t+t_{0}\right) ;$

it gives:

$$
4 \Gamma \frac{\partial C}{\partial \Gamma}=\frac{\partial}{\partial z}(1+\gamma f(C)) \frac{\partial C}{\partial z}+2 z \frac{\partial C}{\partial z}
$$

which is functionally the same equation.

This invariance has an important consequence. As mentioned earlier, since one will need to handle an arbitrary initial state, even for a non-linear analysis:

$$
\begin{aligned}
& C_{R}\left(x, t_{R}\right)=\sum B_{m} H_{2 m}\left(x / x_{R}\right) \exp -\left(x / x_{R}\right)^{2}, \quad \int C_{R}\left(x, t_{R}\right) d x=\hat{1} \\
& C_{F}\left(x, t_{F}\right)=\sum \tilde{B}_{m} H_{2 m}\left(x / x_{F}\right) \exp -\left(x / x_{F}\right)^{2}, \int C_{F}\left(x, t_{F}\right) d x=\hat{1}
\end{aligned}
$$

If one chooses $\Gamma=1+4 D_{0}\left(t-t_{R}\right) / x_{R}{ }^{2}$, then $\Gamma \rightarrow 1$ as $t \rightarrow t_{R}$ and also:

$$
z^{2}=x^{2} / 4 D_{0}\left(t+t_{0}\right) \equiv x^{2} / \Gamma x_{R}^{2}
$$

At $\left(t=t_{F}\right), \Gamma \rightarrow 1+4 D_{0}\left(t_{F}-t_{R}\right) / x_{R}{ }^{2}$, and because $\Gamma$ is linear in either the time variable or in $\mathrm{D}_{0}$, one can view the situation as this: The initial state needs $\Gamma_{R}=1$ and the final state needs $\Gamma_{F}=1+\left(4 D_{0}\right)$. $\left(t_{F}-t_{R}\right) / x_{R}^{2}$, whether one is doing a linear or non-linear equation 
analysis, since ' $\Gamma$ ' is analogous to a time-variable. Since one measures a $C_{R}\left(x, t_{R}\right)$ and a $C_{F}\left(x, t_{F}\right)$, suppose the value of $D_{0}$, and the form of $D(C)$ were known, but not the value of $t_{F}$, one could search the entire range of time $\left(t=\left[t_{R}, \infty\right]\right)$, calculating:

$$
\Gamma(t)=1+4 D_{0}\left(t-t_{R}\right) / x_{R}^{2}
$$

and for each. value of $\Gamma$, one could get a propagated forward state from $C_{R}\left(x, t_{R}\right)$ (given one has solved the $D(C)$ problem) which is denoted $\hat{C}_{R}(x, t)$. Then by comparing $\hat{C}_{R}(x, t)$ to $C_{F}\left(x, t_{F}\right)$ : for a range of values of ' $t$ ', the best match must occur at $\left(t=t_{F}\right)$, where $\Gamma$ will have the value $\Gamma_{F} \cdot$ Or,alternately, $D_{0}$ could be viewed as an unknown and $\left(t_{R}, t_{F}\right)$ as known; then by searching the entire spectrum of $D$ :

$$
\Gamma(D)=1+4 D\left(t_{F}-t_{R}\right) / x_{R}^{2}
$$

the closest comparison between the propagated-forward state and the measured final state again must occur at $\left(\Gamma=\Gamma_{F}\right)$, i.e., at $\left(D=D_{0}\right)$.

This means that instead of having $(z, t)$ as dynamical variables and $\left(\gamma, D_{0}\right)$ as parameters (given $\left.D(C)=D_{0}[1+\gamma f(C)].\right)$, one can have $\left(z, D_{0}\right)$ as dynamical variables, and $\left(y, t_{F}-t_{R}\right)$ as parameters. Since:

$$
4 \Gamma \frac{\partial C}{\partial \Gamma}=\frac{\partial}{\partial z}(1+\gamma f(C)) \frac{\partial C}{\partial z}+2 z \frac{\partial C}{\partial z} .
$$

embodies the most general concentration dependent diffusion equation, we will for the rest of this thesis use $\Gamma=\Gamma(D)$, where

$$
\begin{aligned}
& \Gamma(D)=1+4 D\left(t_{F}-t_{R}\right) / x_{R}{ }^{2} \\
& z^{2}=x^{2} /\left(x_{R}^{2}+4 D\left(t_{F}-t_{R}\right)\right)=x^{2} / \Gamma x_{R}{ }^{2}
\end{aligned}
$$

The initial state now is the $D \rightarrow 0 \cdot 1$ imit, and the final state is $D \rightarrow D_{0} \quad$ (thereby determining $D_{0}$, for a linear or non-linear equation). 


$$
\mathrm{V} \cdot \text {. On } D(C)=\mathrm{D}_{\mathrm{o}}(1+\alpha C)
$$

In terms of $(z, \Gamma)$, the $D(C)=D_{0}(1+\alpha C)$ problem gives:

$$
4 \Gamma \cdot \frac{\mathrm{dC}}{\mathrm{d} \Gamma}=\left(\frac{\partial^{2} \mathrm{C}}{\partial z^{2}}\right)+2 z \frac{\partial \mathrm{C}}{\partial z}+\alpha\left[\mathrm{C}\left(\frac{\partial^{2} \mathrm{C}}{\partial z^{2}}\right)+\left(\frac{\partial \mathrm{C}}{\partial z}\right)^{2}\right] .
$$

In the $(\alpha=0)$ case, this has a solution

$$
\begin{aligned}
& C(z, \Gamma)=\sum G_{m}(\Gamma) H_{2 m}(z) \text { exp- } z^{2} \\
& G_{m}(\Gamma)=B_{m} / \Gamma(m+1 / 2)
\end{aligned}
$$

where $q_{m} 2 H_{2 m}(z)$ exp- $z^{2}$ solves the equation:

$$
\left(\frac{\partial}{\partial z}\right)^{2} q_{m}+2 z\left(\frac{\partial}{\partial z}\right) q_{m}+2(2 m+1) q_{m}=0
$$

One may at first hope that a more general form of $G_{m}(\Gamma)$ might solve the non-linear equation, but expecting that the non-linearities would couple various orthogonal-function-set elements, this would at best give some totally coupled (probably non-linear) set of equations for the set $G_{m}(\Gamma)$. Indeed, some method to decouple the non-linearities is necessary. The non-linear terms being inherently "quadratic" in ' $\mathrm{C}$ ', will tend to give terms vexp- $\left(2 z^{2}\right)$. The introduction of exp- $\left(2 z^{2}\right)$ terms in $C(z, \Gamma)$ will then create in the non-linear part, terms that go as $\exp -\left(3 z^{2}\right)$, etc:, etc. Thus, a minimum spanning space of functions is:

$$
C(z, \Gamma)=\sum_{n=1}^{\infty} \sum_{m=0}^{\infty} g_{m, n}(\Gamma) H_{2 m}(\sqrt{n} z) \exp -\left(n z^{2}\right) .
$$

One must be cautioned that these are no longer linearly independent. For each given ' $\mathrm{n}$ ', the set $g_{\mathrm{m}, \mathrm{n}}$ (for different $\mathrm{m}$ ) are linearly independent, but any given $g_{m, n}$ term is not linearly independent of any $\left(g_{m^{\prime}, n^{\prime}}\right)$ term 
for any $\left(n \neq n^{\prime}\right)$. We, henceforth, drop the explicit argument of $\Gamma$ in $\mathrm{g}_{\mathrm{m}, \mathrm{n}}(\Gamma)$ and define:

$$
g_{m, n}(\Gamma) \equiv g(m, n), \frac{d}{d \Gamma} g(m, n) \equiv \dot{g}(m, n) .
$$

If, indeed, such a function set solves the non-linear-equation, just because it is written as a sum of terms $\bar{A}_{i}(\Gamma) \bar{E}_{i}(z)$, the original equation still will not be separable (i.e., $L_{1} \bar{A}_{i}(\Gamma)=0$ and $L_{2} \bar{E}_{i}(z)=0$ where ' $\mathrm{L}_{1}$ ' is a differential operator in $\Gamma$, and ' $\mathrm{L}_{2}$ ' a differential operator in z) unless each term $\bar{A}_{i} \bar{E}_{i}$ by itself is a solution to the equation. In short, if $\left(\bar{A}_{1}(\Gamma) \bar{E}_{1}(z)+\bar{A}_{2}(\Gamma) \bar{E}_{2}(z)\right)$, is a solution to an equation where $\bar{A}_{1}(\Gamma) \bar{E}_{1}(z)$ is not, the differential equation will not be separable in $(z, \Gamma)$. Thus, without even attempting to separate variables, and defining:

$$
q(2 m ; n) \equiv H_{2 m}(z \sqrt{n}) \exp -\left(n z^{2}\right), \quad g(-1, n) \equiv 0
$$

direct substitution of the series for ' $C$ ' into the non-1inear equation gives:

$$
\begin{aligned}
& 4 \Gamma \frac{\partial C}{\partial \Gamma}-\left(\frac{\partial^{2} C}{\partial z}\right)-2 z \frac{\partial C}{\partial z}= \\
& \sum_{n=1}^{\infty} \sum_{m=0}^{\infty}[4 \Gamma \dot{g}(m, n)+2(2 m+1) g(m, n)-(n-1) g(m-1, n)] q(2 m, n) \\
& \alpha\left(C\left(\frac{\partial^{2} C}{\partial z^{2}}\right)+\left(\frac{\partial C}{\partial z}\right)^{2}\right)=\alpha \sum_{n 1, n 2=1}^{\infty} \sum_{m 1, m 2=0}^{\infty} g(m 1, n 1) g(m 2, n 2) \\
& {[.5(n 1) \cdot q(2 m 1+2, n 1) q(2 m 2, n 2)+.5(n 2) q(2 m 1, n 1) q(2 m 2+2, n 2)} \\
& \left.\quad+(n 1)^{1 / 2}(n 2)^{1 / 2} q(2 m 1+1, n 1) q(2 m 2+1, n 2)\right]
\end{aligned}
$$

Defining $a^{2}=(n 1 / n), \quad b^{2}=(n 2 / n)$, and equating coefficients of $\left(\exp -n z^{2}\right)$ 
gives:

$$
\begin{aligned}
& \sum_{m=0}^{\infty}[4 \Gamma \dot{g}(m, \mathfrak{n})+2(2 m+1) g(m, n)-(n-1) g(m-1, n)] q(2 m, n)= \\
& (a n / 2) \sum_{n 1, n 2=1 m l, m 2=0}^{\infty} \delta\left(a^{2}+b^{2}=1\right) \delta\left(n a^{2}=n 1\right) \delta\left(n b^{2}=n 2\right) g(m 1, n 1) g(m 2, n 2) \times \\
& \quad\left[a^{2} q\left(2 m 1+2, n a^{2}\right) q\left(2 m 2, n b^{2}\right)+b^{2} q\left(2 m 1, n a^{2}\right) q\left(2 m 2+2, n b^{2}\right)\right. \\
& \left.+2 a b q\left(2 m 1+1, n a^{2}\right) q\left(2 m 2+1, n b^{2}\right)\right]
\end{aligned}
$$

Multiplying both sides by $\mathrm{H}_{2 \mathrm{p}}(z \sqrt{n}) \mathrm{d}(z \sqrt{n})$ and integrating, this will. isolate a separate equation for each $g(p, n)$ function:

$$
\begin{aligned}
& \int q(2 m, n) H_{2 p}(z \sqrt{n}) d(z \sqrt{n})= \\
& \int H_{2 m}(z \sqrt{n}) H_{2 p}(z \sqrt{n}) \exp -\left(n z^{2}\right) d(z \sqrt{n})=\delta(m=p) \pi{ }^{1 / 2}(2 p) ! 2^{2 p} \\
& {[4 \Gamma \dot{g}(p, n)+2(2 p+1) g(p, n)-(n-1) g(p-1, n)]=} \\
& (\alpha n / 2) \sum_{n 1, n 2=1}^{\infty} \sum_{1, m 2=0}^{\infty} \delta(n 1+n 2=n) g(m 1, n 1) g(m 2, n 2) \theta(m 1, m 2, n 1, n 2 ; p, n)
\end{aligned}
$$

where:

$$
\begin{aligned}
& \Theta(m 1, m 2, n 1, n 2 ; p, n)=\left(\pi^{1 / 2}(2 p) ! 2^{2 p}\right)^{-1} \delta\left(n 1=n a^{2}\right) \delta\left(n 2=n b^{2}\right) \times \\
& \int \delta\left(a^{2}+b^{2}=1\right) \cdot a^{2} H_{2 m 1+2}(a \xi) H_{2 m 2}(b \xi)+b^{2} H_{2 m 1}(a \xi) H_{2 m 2+2}(b \xi) \\
& \left.\quad+2 a b H_{2 m 1+1}(a \xi) H_{2 m 2+1}(b \xi)\right] H_{2 p}(\xi) \exp -\left(\xi^{2}\right) d \xi
\end{aligned}
$$

The $\theta(\mathrm{m} 1, \mathrm{~m} 2, \mathrm{n} 1, \mathrm{n} 2 ; \mathrm{p}, \mathrm{n})$ is a difficult integral, and it is calculated explicitly in Appendix V. What is noteworthy is that all the arguments of $\theta(\ldots)$ are integer indices, and it is a pure number depending on no physical parameters. It is analogous to a mode-mode coupling whereby the non-linearity $(\alpha)$ couples together the modes $\left(q_{m} \sim H_{2 m}(z)\right.$ exp- $\left.z^{2}\right)$ of the linear system $\left(\frac{\partial}{\partial t} q_{m}=D_{0}\left(\frac{\partial}{\partial x}\right)^{2} q_{m}\right)$ with different strengths. 


\section{Fundamental Properties of} the $g(p, n)-$ Equations

Having begun with:

$$
C(z, \Gamma)=\sum_{n=1}^{\infty} \sum_{m=0}^{\infty} g(m, n) H_{2 m}(z \sqrt{n}) \exp -\left(n z^{2}\right),
$$

the 'fundamental equation':

$$
\begin{aligned}
& {[4 \Gamma \dot{g}(p, n)+2(2 p+1) g(p, n)-(n-1) g(p-1, n)]=} \\
& (\alpha n / 2) \underset{n 1, n 2-m 1, m 2}{\sum} \delta(n 1+n 2=n) g(m 1, n 1) g(m 2, n 2) \theta(m 1, m 2, n 1, n 2 ; p, n),
\end{aligned}
$$

without further calculation, mirrors practically all the basic physics of the non-linear diffusion.

The set: of functions $g(p, n)$ can be arranged in an array-like form, with each 'p'-1abel being a column, each 'n'-label being a row:

$$
\begin{array}{llll}
g(0,1) & g(1,1) & g(2,1) & g(3,1) \ldots \\
g(0,2) & g(1,2) & g(2,2) & \ldots \\
g(0,3) & g(1,3) & \ldots
\end{array}
$$

The $\delta(n 1+n 2=n)$ restriction means that each $g(p, n)$. function depends only on the rows before the $n^{\text {th }}$-row, and not on any row after the $n^{\text {th }}$-row. The $g(p-1, n)$ term in the equation of $g(p, n)$ means that each element in the $n^{\text {th }}$-row also depends on its neighbor to the immediate left, and no other. The system is recursive. One can "bootstrap" this solution by calculating the first row, which in turn determines the second, both determining the third row; etc. It is by such a mechanism that this (non"linearly independent") set of functions decouples the non-linearity of the $D(C)$ equation. 
Since the only place the non-linearity parameter $(\alpha)$ appears is as a coefficient of the entire right-hand-side of Eq. 4.46 and because of the $\delta(n 1+n 2=n)$ restriction, each $g(p, n) \sim(\alpha)^{n-1}$. This means that $C(z, \Gamma)$ may be written as:

$$
C(z, \Gamma)=\phi_{0}(z, \Gamma)+\alpha \phi_{1}(z, \Gamma)+\alpha^{2} \phi_{2}(z, \Gamma)+\cdots
$$

The $g(p, 1)$ functions are "special" in the sense that the $\delta(n 1+n 2=n)$ restriction can never be satisfied for $\quad(n=1)$ (since both $n 1$ and $n 2$ begin at 1). Thus, the entire right-hand-side vanishes for $(n=1)$. The ' $(n-1) g(p-1, n)$ ' term. also vanishes when $(n=1)$, and one is left with:

$$
4 \Gamma \dot{g}(p, 1)+2(2 p+1) g(p, 1)=0
$$

Each element of the first row is therefore independent, and easily:

$$
g(p, 1)=B_{p} / \Gamma^{p+1 / 2}
$$

The first row exactly matches the solution to the linear equation. The $B_{p}$ numbers are arbitrary constants, and thus they allow an arbitrary initial state, just as in the linear equation case. Since this formalism is patently analytic, we have now matched two of the basic requirements outlined in Sec. I (Chapter 4).

This 'fundamental equation' is a linear first order differential equation of the form:

$$
4 \Gamma \dot{g}(p, n)+2(2 p+1) g(p, n)=(\text { given functional of } \Gamma) .(4.50)
$$

As such, each $\dot{g}(p, n)$ gives one arbitrary constant $A(p, n)$ where:

$$
B_{p} \equiv A(p, n=1)
$$

The initial state $\left(t \rightarrow t_{R}\right)$ has in general $\Gamma \rightarrow \Gamma_{0}$ (for the particular choice $\Gamma=1+4 D\left(t_{F}-t_{R}\right) / x_{R}^{2}$, with $\left[D \leftrightarrow D_{0}\left(t-t_{R}\right) /\left(t_{F}-t_{R}\right)\right], \Gamma_{0}$ is 
especially simple: $\Gamma_{0}=1$ ), and to preserve the first row converging to the given initial state as $\Gamma \rightarrow \Gamma_{0}$, the additional restriction must be imposed:

$$
\operatorname{Limit}_{\Gamma \rightarrow \Gamma_{0}} g(p, n)=(0 \text { if } n \neq 1)
$$

This can be combined with the $(n=1)$ case to give:

$$
\underset{\Gamma \rightarrow \Gamma}{\operatorname{Limit}} g(p, n)=B_{p} \delta(n=1)
$$

and thus each "arbitrary" constant $A(p, n)$ has been assigned a definite value: The solution is complete. Given a particular initial state, every $g(p, n)$ function is now uniquely specified. Besides the physical parameters $\left(D_{0}, \alpha\right)$. whose values are to be determined from experiments, there are no other hidden arbitrarinesses or covert fitting parameters. Starting with $C_{R}\left(x, t_{R}\right)$, the propagated forward state $\hat{C}_{R}\left(x, t_{F} ; D_{0}, \alpha\right)$ has been totally determined.

$$
* * *
$$

One must finally ask, as the propagated forward state $\hat{C}_{R}\left(x, t_{F} ; D_{0} \alpha\right)$ has been totally determined by this procedure, how one incorporates the final condition of normalization preservation:

$$
\int \mathrm{C}(\mathrm{x}, \mathrm{t}) \mathrm{dx}=\hat{\mathrm{i}} \text { (independent of time) }
$$

(since the given procedure has made, as yet; no reference to this condition). Recalling that the first member of an orthogonal function set has a finite area under it, and no other, and recalling that $c(z, \Gamma)=\sum g(m, n) q(2 m, n)$ is a set of orthogonal function sets, the normalization preservation 
condition: $\int C(z, \Gamma) d x=\hat{l}$ requires:

$$
\begin{aligned}
\hat{1} & =\left(x_{R} \Gamma^{1 / 2}\right) \int C(z, \Gamma) d\left[x /\left(x_{R} \Gamma^{1 / 2}\right)\right]=\left(x_{R} \Gamma^{1 / 2}\right) \int C(z, \Gamma) d z \\
& =\left(x_{R} \Gamma^{1 / 2}\right) \underset{m ; n}{[g(m, n)} \int H_{2 m}(z \sqrt{n}) \exp -\left(n z^{2}\right) d z \\
& =\left(x_{R} \Gamma^{1 / 2}\right) \sum_{(n)} g(0, n)(\pi / n){ }^{1 / 2}
\end{aligned}
$$

Since the $g(0, n)$ equations are some particular functions of ' $\Gamma$ ' given by :

$$
\begin{aligned}
& 4 \Gamma \dot{g}(0, n)+2 g(0, n)= \\
& (\alpha n / 2) \sum_{n 1, n 2 m 1, m 2} \delta \sum_{n} \delta(n 1+n 2=n) g(m 1, n 1) g(m 2, n 2) \Theta(m 1, m 2, n 1, n 2 ; 0, n),
\end{aligned}
$$

it seems that not only is the full (to all orders in $\alpha$ ) solution required so that each $g(0, n)$ as a function of $\Gamma$, can be known, but it also seems that there is no guarantee when one does the sum indicated by Eq. (4.55) that it will indeed be an equality. This problem is compounded by the fact that each $g(0, n) \sim(\alpha)^{n-1}$, giving without further calculation:

$$
\hat{1}=\left(x_{R} \Gamma^{1 / 2}\right) \cdot \sum_{(n)} n^{-1 / 2 \alpha^{n-1}}\left[\operatorname{Term} \text { in }\left(\Gamma, \Gamma_{0}\right)\right]
$$

It appears that $\mathrm{Eq} .(4.57)$ is a contradiction because one cannot alter the values of $(a, \hat{j})$ to fit the mathematics, for both are totally determined beforehand by the physics. Quite a quandry.

The resolution comes in the calculation of $\theta(\mathrm{m} 1, \mathrm{~m} 2, \mathrm{n} 1, \mathrm{n} 2 ; \mathrm{p}, \mathrm{n})$. This function of 5 integer indices for any given ' $p$ ', this strength of mode-coupling function, has the property that (see Appendix V):

$$
\theta(m 1, m 2, n 1, n 2 ; p=0, n)=0
$$

This additional "symmetry" which is nowhere "built-in", not only prevents 
the normalization-requirement from re-coupling together what the rest of this methodology has carefully decoupled into a recursive process, but also it demonstrates that this solution automatically preserves normalization. It is this final property that changes the original ansatz:

$$
C(z, \Gamma)=\sum_{m, n} g(m, n) H_{2 m}(z \sqrt{n}) \exp -\left(n_{z}^{2}\right)
$$

from being an "educated guess," into being the true solution. Thus, the $\mathrm{g}(0, \mathrm{n})$-equations are simply:

$$
4 \Gamma \dot{g}(0, n)+2 g(0, n)=0,
$$

and with the $\Gamma \rightarrow \Gamma_{0}$ condition (Eq. (4.53)), one has finally:

$$
\begin{aligned}
g(0, n) & =\delta(n=1) B_{0} / \Gamma^{1 / 2} . \\
& * * * *
\end{aligned}
$$

Next, performing the transformation:

$$
g(p, n)=K(p, n) \Gamma^{-(p+1 / 2)} e \cdot(p, n)
$$

where $e(p, n)$ is a function of $\Gamma$ and $k(p, n)$ are constants, one transforms

$$
[4 \Gamma \dot{g}(p, n)+2(2 p+1) g(p, n)-(n-1) g(p-1, n)]=\alpha\{\text { non-1inear part }\}
$$

into:

$$
\Gamma^{-(p-1 / 2)}[4 K(p, n) \dot{e}(p, n)-(n-1) K(p-1, n) e(p-1, n)]=\alpha\{\text { non-1inear part }\}
$$

For $(n=1)$, choosing $(K(p, 1)=1)$; and for $(n \neq 1)$, choosing:

$$
\frac{K(p, n)}{K(p-1, n)}=\frac{(n-1)}{4}, \quad K(0, n)=1
$$


one gets, valid for all $(p, n)$ :

$$
K(p, n)=[.25(n-1)+\delta(n=1)]^{P} \text {. }
$$

This gives:

$$
\begin{aligned}
& {[\dot{e}(p, n)-(1-\delta(n=1)) e(p-1, n)]=.25\{4 /[(n-1)+.4 \delta(n=1)]\}^{p} \Gamma^{p-1 / 2} \times} \\
& (\alpha n / 2) \sum_{n 1, n 2 m 1, m 2} \sum_{j(n 1+n 2=n)[.25(n 1-1)+\delta(n 1=1)]^{m 1}}[.25(n 2-1)+\delta(n 2=1)]^{m 2} \\
& x \cdot\left\{\theta(m 1, m 2 ; n 1, n 2 ; p, n) e(m 1, n 1) e(m 2, n 2) / \Gamma^{m 1+m 2+1}\right\}
\end{aligned}
$$

The ( $n=1)$ case is trivial, giving:

$$
\begin{aligned}
\dot{e}(p, 1)=B_{p}, \quad g(p, 1) & =B_{p} / \Gamma^{p+1 / 2} \\
& * * *
\end{aligned}
$$

For $(n \neq 1)$, one has the system of equations:

$$
\dot{e}(p ; n)-e(p-1, n)=\hat{\mathbf{f}}^{-1}(p, n ; \Gamma)
$$

subject to:

$$
\underset{\Gamma \rightarrow \Gamma \text { Limit }}{\operatorname{Lin}} \operatorname{e}(\mathrm{p}, \mathrm{n})=0
$$

If :

$$
\hat{f}^{q}(p, n ; \Gamma)=\left[(q+1)^{t h} \text { indefinite integral of } \hat{f}^{-1}(p, n ; \Gamma)\right]
$$

one can verify that the set $e(p, n)$ has the following solution (derived by trial-and-error method):

$$
e(p, n)=\sum_{q=0}^{p-1} \hat{f}^{q}(p-q, n ; \Gamma)-\sum_{q=0}^{p-1}\left[\left(\Gamma-\Gamma_{0}\right)^{q} / q !\right]\left[\sum_{r=0}^{p-q-1} \hat{f}^{r}\left(p-q-r, n ; \Gamma_{0}\right)\right]
$$

Since this solution has a part representing successive multiple-integrals 
and a part which is a polynomial is $\Gamma$, one can alternately try:

$$
\begin{aligned}
& \tilde{f}^{q}(p, n ; \Gamma)=\int_{\left(\Gamma_{0}\right)}^{(\Gamma)} \cdots \hat{f}^{-1}(p, n ; \Gamma)(d \Gamma)^{q+1} \equiv \\
& \int_{\Gamma_{1}=\Gamma_{0}}^{\Gamma} \mathrm{d} \Gamma_{1} \quad \int_{\Gamma_{2}=\Gamma_{0}}^{\Gamma} \mathrm{d} \Gamma_{2} \quad \cdots \int_{(q+1)}^{\Gamma} \int_{0}^{\Gamma} \mathrm{d} \Gamma(q+1) \hat{\mathrm{f}}^{-1}\left(\mathrm{p}, \mathrm{n} ; \Gamma_{(q+1)}\right)
\end{aligned}
$$

This has the property that $\tilde{f}^{q}\left(p, n ; \Gamma_{0}\right)=0$ and:

$$
\left(\frac{d}{d \Gamma}\right)^{m}\left[\tilde{f}^{q}(p, n ; \Gamma)\right]=\tilde{f}^{q-m}(p, n ; \Gamma)
$$

where, of course, $\tilde{f}^{-1}(p, n ; \Gamma) \equiv \hat{f}^{-1}(p, n ; \Gamma)$. One now tries

$$
e(p, n)=\sum_{q=0}^{p-1} \cdot \tilde{f}^{q}(p-q, n ; \Gamma)-\sum_{q=0}^{p-1}\left[\left(\Gamma-\Gamma_{o}\right)^{q} / q !\right] \cdot \tilde{K}\left(q, p, \tilde{n} ; \Gamma_{o}\right) .
$$

Applying $e(p, n)=0$ at $\Gamma_{l}=\Gamma_{0}$, one gets:

$$
\tilde{K}\left(0, p, n ; \Gamma_{0}\right)=0
$$

Substituting Eq. (4.75) into Eq. (4.69) gives that this is indeed a solution also; providing

$$
\tilde{K}\left(q ; p-1, n ; \Gamma_{o}\right)=\tilde{K}\left(q+1, p, n ; \Gamma_{0}\right)
$$

This means that by Eq. (4.76) the entire set $\tilde{K}\left(q, p, n ; \Gamma_{0}\right)=0$ and:

$$
e(p, n)=\sum_{q=0}^{p-1} \tilde{f}^{q}(p-q, n ; \Gamma)
$$

Next, one may ask what functions make up $e(p, n)$ ? One starts with $e(p, 1)=$ constant; $\hat{f}^{-1}(p, 2 ; \Gamma) \sim\left[1 / \Gamma^{n+1 / 2}\right.$ (n=integer) $]$, so $e(p, 2)$ contains integrals of $\left(1 / \Gamma^{n+1 / 2}\right)$ and gives $e(p, 2) \sim\left(1 / \Gamma^{n+1 / 2}\right)$. This gives $\hat{f}^{-1}(p, 3 ; \Gamma) \sim\left[1 / \Gamma^{n}(n=\right.$ integer $\left.)\right]$, so $e(p, 3)$ contains integrals of $\left[1 / \Gamma^{n}\right.$ (n=integer) $]$, and thus it will develop a $\ln (\Gamma)$-term. Extending 
and a part which is a polynomial is $\dot{\Gamma}$, one can alternately try:

$$
\begin{aligned}
& \tilde{f}^{q}(p, n ; \Gamma)=\int_{\left(\Gamma_{0}\right)}^{(\Gamma)} \cdots \hat{f}^{-1}(p, n ; \Gamma) \cdot(d \Gamma)^{q+1} \equiv
\end{aligned}
$$

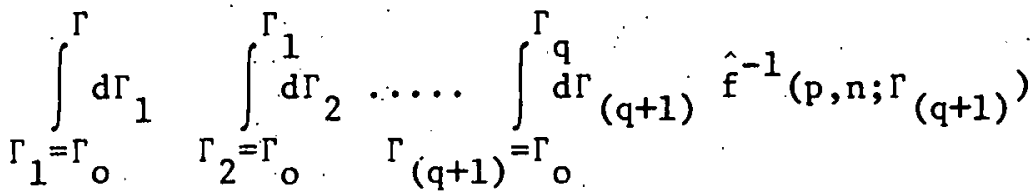

This has the property that $\tilde{f}^{q}\left(p, n ; r_{0}\right)=0$ and:

$$
\left(\frac{d}{d \Gamma}\right)^{m}\left[\tilde{f}^{q}(p, n ; \Gamma)\right]=\tilde{f}^{q-m}(p, n ; \Gamma)
$$

where, of course, $\tilde{f}^{-1}(p, n ; \Gamma) \equiv \hat{f}^{-1}(p, n ; \Gamma)$. One now tries

$$
e(p, n)=\sum_{q=0}^{p-1} \sum^{q}(p-q, n ; \Gamma)-\sum_{q=0}^{p-1}\left[\left(\Gamma-\Gamma_{0}\right)^{q} / q !\right] \cdot \tilde{k}\left(q, p, n ; \Gamma_{0}\right) .
$$

Applying $e(p, n)=0$ at $\Gamma=\Gamma_{0}$, one gets:

$$
\tilde{\mathrm{K}}\left(0, \mathrm{p}, \mathrm{n} ; \Gamma_{\mathrm{o}}\right)=0
$$

Substituting Eq. (4.75) into Eq. (4.69) gives that this is indeed a solution also; providing

$$
\tilde{\mathrm{K}}\left(\mathrm{q}, \mathrm{p}-1, \mathrm{n} ; \tilde{\Gamma}_{\mathrm{o}}\right)=\tilde{\mathrm{K}}\left(\mathrm{q}+1, \mathrm{p}, \mathrm{n} ; \Gamma_{\mathrm{o}}\right)
$$

This means that by Eq. $(4.76)$ the entire set $\tilde{K}\left(q, p, n ; \Gamma_{0}\right)=0$ and:

$$
e(p, n)=\sum_{q=0}^{p-1} \tilde{f} q(p-q, n ; r)
$$

Next, one may ask what functions make up $e(p, n)$ ? One starts with $e(p, 1)=$ constant; $\hat{f}^{-1}(p, 2 ; \Gamma) \sim\left[1 / \Gamma^{n+1 / 2}\right.$ (n=integer) $]$, so $e(p, 2)$ contains integrals of $\left(1 / \Gamma^{n+1 / 2}\right)$ and gives $e(p, 2) \sim\left(1 / \Gamma^{n+1 / 2}\right)$. This gives $\hat{\mathrm{f}}^{-1}(\mathrm{p}, 3 ; \Gamma) \sim\left[1 / \Gamma^{\mathrm{n}}(\mathrm{n}=\right.$ integer $\left.)\right]$, so $\mathrm{e}(\mathrm{p}, 3)$ contains integrals of $\left[1 / \Gamma^{n}\right.$ (n=integer)], and thus it will develop a $\ln (\Gamma)$-term. Extending 
such analyses tọ. $e(p, n)$, one finds that every term in $e(p, n)$ must be of the general form

$$
\begin{aligned}
& e(p, n) \sim(\Gamma)^{\nu}(\ln (\Gamma))^{m} \\
& \nu=\text { (integer and/or half-integer), } m=\text { (integer). }
\end{aligned}
$$

Thus an important function will be:

$$
\Phi\left(\nu, \mathrm{m}, q ; \Gamma, \Gamma_{0}\right)=\int_{\left(\Gamma_{0}\right)}^{(\Gamma)} \ldots \int^{\nu} \Gamma^{\nu}(\ln (\Gamma))^{m}(\mathrm{~d} \Gamma)^{q+1}
$$

This is worked out explicitly in Appendix IV.

\section{Recoupling}

Having seen that:

$$
C(z, \Gamma)=\sum g(p ; n) H_{2 p}(z \sqrt{n}) \exp -\left(n z^{2}\right)
$$

does indeed solve the $D(C)=D_{0}(1+\alpha C)$ problem, we note that the measured initial and final states:

$$
\begin{aligned}
C\left(x, t_{R}\right) & =\sum_{m=0}^{N I} B_{m} H_{2 m}\left(\frac{x}{x_{R}}\right) \exp -\left(\frac{x}{x_{R}}\right)^{2} \\
C \cdot\left(x, t_{F}\right) & =\sum_{m=0}^{N F} \tilde{B}_{m} H_{2 m}\left(\frac{x}{x_{F}}\right) \exp -\left(\frac{x}{x_{F}}\right)^{2}
\end{aligned}
$$

present only a single array of orthogonal functions.. Since the $\tilde{B}_{m}$ parameters totally specify the final state, Eq. (4.81) can now be reexpressed in terms of a single series:

$$
C(z, \Gamma)=\sum_{p=0}^{\infty} G_{p}(\Gamma) H_{2 p}(z) \text { exp- } z^{2}
$$

Before this is actually done, it must be demonstrated that a given series, $H_{2 p}(z \sqrt{n}) \cdot \exp -\left(n z^{2}\right)$, can always be re-expressed as a single 


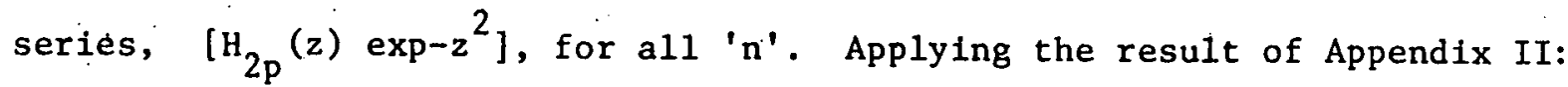
If a function has a tail that decreases as fast as or faster than A-exp$\left(\mathrm{x} / \mathrm{x}_{\mathrm{A}}\right)^{2}$, it can be expressed as a converging series in exp- $\left(\mathrm{x} / \mathrm{x}_{\mathrm{R}}\right)^{2}$ if $\left(2^{-1 / 2} x_{A}\right)<x_{R}<\infty ; 1$ letting $+n z^{2}=\left(x / x_{A}\right)^{2}$ and $z^{2}=\left(x / x_{R}\right)^{2}$, this requires of ' $n$ ':

$$
(2 n)^{-1 / 2}<1<\infty
$$

i.e., $n \geq .7071 \ldots$, but, since ' $n$ ' is a positive integer in this formalism, this criterion is always satisfied.

Each higher, ' $n$ !' function set in $\left[g(p, n) H_{2 p}(\sqrt{n z}) \cdot \exp -\left(n z^{2}\right)\right]$. is analogous to higher and higher "harmonics", as are larger and larger 'p' values even for the "fundamental" set: $\left[g(p, 1) \cdot H_{2 p}(z)\right.$ exp- $\left.z^{2}\right]$. Both large p-values and large n-values correspond to functions which tend to oscillate at a greater and greater frequency. Thus, although the representation

$$
C(z, \Gamma)=\sum g(p, n) H_{2 p}(z \sqrt{n}) \exp -\left(n z^{2}\right)
$$

is "exact", to sum first in ' $p$ ' up to some $p(\max )$, and then to sum in ' $n$ ', is to introduce terms that may correspond to higher "harmonics" beyond $p(\max )$. Thus, as long as one has a finite number of polynomials in both measured initial and final states, one needs to change $c(z, \Gamma)$ from the $g(p, n)$ representation into the $G_{p}(\Gamma)$ representation, so that one is preserving equivalent levels of oscillation approximation. This procedure will not alter the intrinsic normalization preservation of the solution. Detailed, it is thus: One seeks $G_{p}(\Gamma)$ in the following:

$$
C(z, \Gamma)=\sum_{p=0}^{\infty} G_{p}(\Gamma) H_{2 p}(z) \exp -z^{2} \equiv \sum_{n=1}^{\infty} \sum_{p=0}^{\infty} g(p, n) H_{2 p}(z \sqrt{n}) e^{-n z^{2}}
$$


Multiplying both sides by $\mathrm{H}_{2 \mathrm{~m}}(\mathrm{z}) \mathrm{dz}$ and integrating:

$$
\begin{gathered}
\pi^{1 / 2}(2 m) ! 2^{2 m} \cdot G_{m}=\sum_{n=1}^{\infty} \sum_{p=0}^{\infty} g(p, n) \int H_{2 m}(z) H_{2 p}(z \sqrt{n}) \exp -\left(n z^{2}\right) \cdot d z= \\
\sum_{n=1}^{\infty} \sum_{p=0}^{\infty} g(p, n) \frac{1}{\sqrt{n}} \int H_{2 m}(a \xi) H_{2 p}(\xi) \exp -\left(\xi^{2}\right) d \xi \delta\left(n a^{2}=1\right)
\end{gathered}
$$

Using the integral ${ }^{46-49}$ :

$$
\int H_{2 m+n}(a x) H_{n}(x) \exp -\left(x^{2}\right) d x=\pi^{1 / 2} 2^{n} \cdot\left(a^{2}-1\right)^{m} a^{n}(2 m+n) ! / m !
$$

gives:

$$
\begin{aligned}
& 2^{2 m}(2 m) ! G_{m}= \\
& \sum_{n=1}^{\infty} \sum_{p=0}^{\infty} g(p ; n) \delta\left(n a^{2}=1\right) a\left[2^{2 p} \delta(m>p)\left(a^{2}-1\right)^{m-p} a^{2 p}(2 m) ! /(m-p) !\right] \\
& G_{m}(\Gamma)=\sum_{n=1}^{\infty} n^{-m-1 / 2} \sum_{p=0}^{m} g(p, n)[(1-n) / 4]^{m-p} /(m-p) !
\end{aligned}
$$

\section{Review and Extensions}

Thus :

$$
C(z, \Gamma)=\sum_{m=0}^{m(\max )} G_{m}(\Gamma) H_{2 m}(z) \exp -z^{2}
$$

embodies a complete solution to the $D(C)=D_{0}(1+\alpha C)$ problem. If an initial state is represented by:

$$
\sum_{m=0}^{N I} B_{m} H_{2 m}\left(x / x_{R}\right) \exp -\left(x / x_{R}\right)^{2},
$$

then if $D=$ constant, $m(\max ) \equiv$ NI. But for $D=D(C), m(\max )$, in principle, extends to infinity. However, the $G_{m}(\Gamma)$. (for $m>N I$ ) rely on 
implicitly setting $B_{m}=0$ (for $m>N I$ ). Thus, to maintain equivalent levels of approximation in the data fit and the analysis, we will consistently choose $m(\max )$ close to 'NI' as a cutoff.

As the $G_{m}(\Gamma)$ are totally coupled functions of the initial state parameters, $\left\{B_{m} ; m=(0, N I)\right\}$, the associated functions $g(p, n) \sim(\alpha)^{n-1}$ must first be calculated where:

$$
G_{m}(\Gamma)=\sum_{n=1}^{n(\max )} n^{-m-1 / 2} \sum_{p=0}^{m} g(p, n)[(1-n) / 4]^{m-p} /(m-p) ! \ldots
$$

In principle, $n(\max )$ also extends to infinity, but a cutoff for $n$ (max) represents a solution of the $D(C)=D_{0}(1+\alpha C)$ problem where ' $C$ ' is calculated to order $\alpha^{(n(\max )-1)}$.

Since the $G_{m}(\Gamma)$ requires only the calculation $\{g(p, n) ; p=$ $(0, m(\max )\}$, one could do a calculation assuming $g(p, n)=0$ if $p>m(\max )$. But since each, $g\left(p^{\prime}, n^{\prime}\right)$. function in turn determines $\left(g(p, n), n^{\prime}<n\right)$, this truncation might cause an error buildup. Thus, the method that will be used is to calculate $g\left(p^{\prime}, n^{\prime}\right)$ for $p^{\prime}=(0, p(\max )$ ) where $p(\max )>\operatorname{m}(\max )$ This means that each $g\left(p^{\prime}, n^{\prime}\right)$ function will be accurately known when it is substituted into the $g(p, n)$ function.

An additional note: When one examines the details of the $g(p, n)$ functions (see Chapter 6), the gaussian initial state is a calculation of equivalent complexity as an arbitrary initial state. Each "step" of the calculation must still be done, but (given a gaussian) the stages will.be shorter. Because of the non-linearities inherently coupling the eigen functions of the linear equation, one could have expected no other advantage by trying to solve a special case (gaussian) instead of the general case. 
The $g(p, n)$ functions can be recursively calculated; and this solution method not only automatically conserves total mass: $\int C(x, t) d x=\hat{I}$, but, also, it allows an arbitrary initial state function.

A second transformation:

$$
g(p ; n)=[.25(n-1)+\delta(n=1)]^{p} \Gamma^{-(p+1 / 2)} e(p, n)
$$

results in the system of equations:

$$
\dot{e}(p, n)-e(p-1, \dot{n})=\tilde{f}^{-1}(p, n ; \Gamma)
$$

where $\tilde{f}^{-1}(p, n ; \Gamma)$ is a given function of ' $\Gamma$ ' (depending on $e\left(p^{\prime}, n^{\prime}\right)$, $\left.n^{\prime}<n\right)$. This system" was solved with the help of:

$$
\tilde{f}^{q}(p, n ; \Gamma)=\int_{(\Gamma)}^{(\Gamma)} \ldots \int_{0}^{-1}(p, n ; \Gamma)(d \Gamma)^{q+1}
$$

and in terms of this:

$$
e(p, n)=\sum_{q=0}^{p-1} \tilde{f}^{q}(p-q, n ; \Gamma)
$$

The only functions ever to appear in any $\tilde{f}^{-1}(p, n ; \Gamma)$ are functions of the form:

$$
\begin{aligned}
& \tilde{f}^{-1}(p, n ; \Gamma) \sim(\Gamma)^{\nu}(\ln \Gamma)^{m} \\
& \nu=\text { (integer or half-integer), } m=\text { (positive integer) }
\end{aligned}
$$

thus, every $\tilde{f} q(p, n ; \Gamma)$ can be expressed in terms of :

$$
\Phi\left(\nu, m, q ; \Gamma, \Gamma_{0}\right)=\int_{\left(\Gamma_{0}\right)}^{(\Gamma)} \ldots(\Gamma)^{\nu}(\ln \Gamma)^{m}(d \Gamma)^{q+1}
$$

This system of integrals is worked out explicitly in Appendix IV. Thus, one only needs to do the algebra problem of writing down $\tilde{f}^{-1}(p, n ; \Gamma)$ and, just by substitution into given formulae, the $g(p, n)$ functions can be found. 
Just as every $\tilde{f}^{-1}(p, n ; \Gamma)$ (i.e., a calculation of $c(z, \Gamma)$ to any arbitrarily order in $\alpha$ ) involved the same functions $(\Gamma)^{\nu}(\ln \Gamma)^{m}$, every $\tilde{f}^{-1}(p, n ; \Gamma)$ also involves a big (pure number) coupling coefficient denoted by:

$$
\theta(m 1, m 2, n 1, n 2 ; p, n)
$$

$\Theta(\ldots)$ has no dynamical variables in it, and no physical parameters. It too, (since it corresponds to a large integral) has been calculated explicitly (in Appendix V), and it is in a form usable to all orders in $\alpha$ (i.e., all values of $n$ ); thus again, this procedure involves only substitution of a given formula.

The partial differential equation with $D(C)=D(1+\alpha C)$ is thus reduced to an algebra problem with successive substitutions. There are no hidden integrals to be done, no covert couplings, no unseen differentiations, and no further approximations.

$$
* * *
$$

The procedure chosen for beyond this point is the following: Whereas one could now explicitly write down the exact expressions for $\mathrm{n}=(1,2,3 \ldots) \quad\left(\right.$ i.e., $\mathrm{C}(z, \Gamma)$ to order $\left.1, \alpha^{1}, \alpha^{2}, \ldots\right)$, this work aims eventually to solve beyond the $D(C)=D_{0}(1+\alpha C)$ problem. Indeed, the aim is to solve first $D(C)=D_{0}\left(1+\gamma C^{R}\right)$ (R=positive integer), and then further, $D(C)=D_{0}\left(1+\alpha C+B C^{2}+\ldots\right)$ i.e., a diffusivity which is represented as an arbitrary polynomial. For small ' $C$ ', this general formalism would embody the most general $D(C)$ processes. 
Having set down the foundation of a formalism with the $D(C)=$ $D_{0}(1+\alpha C)$ problem, we state the following:

The problem:

$$
\frac{\partial C}{\partial t}=D_{0} \frac{\partial}{\partial x}\left(1+\alpha C+B C^{2}+\ldots\right) \frac{\partial C}{\partial x}
$$

can be converted into one with dynamical variables $(D, z)$ which (exactly as outlined in Sec. IV, Chapter 4) gives:

$$
4 \Gamma \frac{\partial C}{\partial \Gamma}=\frac{\partial}{\partial z}\left(1+\alpha C+\beta C^{2}+\ldots\right) \frac{\partial C}{\partial z}+2 z \frac{\partial C}{\partial z}
$$

The (di-) orthogonal function set:

$$
C(z, \Gamma)=\sum_{n=1}^{\infty} \sum_{m=0}^{\infty} g(m, n) H_{2 m}(z \sqrt{n}) \exp -\left(n z^{2}\right)
$$

also solves this more general problem (with recoupling back into $G_{m}(z) H_{2 m}(z)$ as a final step, as in Sec. VII, Chapter 4). As with the $\mathrm{D}=\mathrm{D}_{\mathrm{o}}(1+\alpha \mathrm{C})$ problem, this more complicated case also conserves mass: $\int C(x, t) d x=\hat{i}$; and an arbitrary function is also allowed as an initial state. The $g(m, n)$ are now determined by the (expanded) fundamental equation:

$$
\begin{aligned}
& {[4 \Gamma \dot{g}(p, n)+2(2 p+1) g(p, n)-(n-1) g(p-1, n)]=} \\
& (a n / 2) \sum \delta(\infty \geq(n 1, n 2) \geq 1) \delta(\infty \geq(m 1, m 2) \geq 0) \delta(n 1+n 2=n) x \\
& {[g(m 1, n 1) g(m 2, n 2)] \theta(m 1, m 2, n 1, n 2 ; p, n)+} \\
& (B n / 3)[\delta(\infty \geq(n 1, n 2, n 3) \geq 1) \delta(\infty \geq(m 1, m 2, m 3) \geq 0) \delta(n 1+n 2+n 3=n) \times \\
& {[g(m 1, n 1) g(m 2, n 2) g(m 3, n 3)] \theta(m 1, m 2, m 3, n 1, n 2, n 3 ; p, n)+} \\
& (\gamma n / 4)[\delta(\infty \geq(n 1, ., n 4) \geq 1) \delta(\infty \geq(m 1 \ldots m 4) \geq 0) \delta(n 1+n 2+n 3+n 4=n) \times \\
& {[g(m 1, n 1) \ldots g(m 4, n 4)] \theta(m 1 \ldots m 4, n 1 \ldots n 4 ; p, n)+\ldots}
\end{aligned}
$$


Some noteworthy points are the following:

(1). The entire (non-linearly coupled) right-hand-side is a determined function of $\Gamma$ once one reaches the stage to calculate $g(p, n)$. Thus, the transformation

$$
g(p, n)=[.25(n-1)+\delta(n=1)]^{p} \Gamma^{-(p+1 / 2)} \text { e }(p, n)
$$

will also reduce this equation to the form

$$
\dot{e}(p, n)-\dot{e}(p-1, n)=\tilde{f}^{-1}(p, n ; \Gamma)
$$

(2). The types of functions which can now appear in $\tilde{f}^{-1}(p, n ; \Gamma)$ are (again) exactly:

$$
(\Gamma)^{\nu}(\ln \Gamma)^{m} \quad\left\{\begin{array}{l}
\nu=\text { integer or half integer } \\
m=\text { positive integer }
\end{array}\right.
$$

Thus the solution:

$$
e(p, n)=\sum_{q=0}^{p-1} \tilde{f}^{q}(p-q, n ; \Gamma)
$$

remains, where each $\tilde{\dot{f}}^{q}(p, n ; \Gamma)$ is composed of $\Phi(\nu, m, q ; \Gamma, \Gamma)$

(3). The $\delta(n 1+n 2+\ldots=n)$ restriction means that a ' $B$ ' first appears starting in $g(p, 3)$; a ' $\gamma$ ' first appears'in $g(p, 4)$, etc., etc.

All the foregoing will be explicitly demonstrated in the next chapter. There, also, will be demonstrated a connection between the various $\odot(\ldots)$ functions of an increasingly larger number of arguments. The end of Chapter 5 will be the same point as here, except that the $D(C)=$ $D_{0}\left(1+\alpha C+B C^{2}+\ldots\right)$ problem will have been reduced to an algebra and substitution situation. We, therefore, defer calculation of the $n=$ $(1,2,3 .$.$) cases explicitly, until Chapter 6$, which will be entirely 
algebra. The explicit construction in Chapter 6 will be for $D=$ $D_{0}\left(1+\alpha C+\beta C^{2}\right)$,

$$
c=\phi_{0}+\alpha \phi_{1}+\alpha^{2} \phi_{2}+\beta \phi_{3}+\ldots
$$

The higher-order terms in 'C' (i.e., an $\alpha^{3}$ term or Ba term) will be neglected because $a D_{0}\left(1+\alpha C+B C^{2}+\gamma C^{3}\right)$ function would give a $\gamma$-contribution in ' $C$ ' of the same order as the higher-order terms of order $\left(\alpha^{3}, B \alpha^{2} \ldots\right)$. Since only algebra will remain, Section IV of Chapter 6 will present everything in terms suitable for direct computer programming. This allows a results-oriented reader to bypass Chapter 5 and all of Chapter 6 up to Section IV. 
CHAPTER 5

THE $\quad D=D_{0}\left(1+\gamma C^{R}\right)$ AND $\quad D=D_{0}\left(1+\alpha C+B C^{2}\right)$

PROBLEMS

\section{General Formalism}

In examining the problem $D(C)=D_{0}\left(1+\gamma C^{R}\right)$, for $(R=0)$, this reduces to a constant diffusivity case but with a $D(C \rightarrow 0) \neq D_{0} \cdot$ Thus, a form $D(C)=D_{0}\left(1+\gamma_{0} \cdot R C^{R}\right)$ is chosen so that as $(R \rightarrow 0), D(C \rightarrow 0)=D_{0}$, giving:

$$
\frac{\partial C}{\partial t}=D_{0} \frac{\partial}{\partial x}\left(1+\gamma_{0} R C^{R}\right) \frac{\partial C}{\partial x}
$$

Transforming variables to $(\Gamma, z)$, as outlined previously (Sec. IV, Chapter 4) gives:

$$
4 \Gamma \frac{\partial C}{\partial \Gamma}=\left(\frac{\partial^{2} C}{\partial z^{2}}\right)+2 z \frac{\partial C}{\partial z}+\gamma_{0} R C^{R-1}\left(C\left(\frac{\partial^{2} C}{\partial z^{2}}\right)+R\left(\frac{\partial C}{\partial z}\right)^{2}\right) .
$$

Again using:

$$
C(z, \Gamma)=\sum_{n=1}^{\infty} \sum_{m=0}^{\infty} g(m, n) H_{2 m}(z \sqrt{n}) \exp -n z^{2}
$$

direct substitution, very similar to the $D(C)=D_{0}(1+\alpha C)$ case, gives:

$$
\begin{aligned}
& \left(4 \Gamma \frac{\partial C}{\partial \Gamma}-\left(\frac{\partial^{2} C}{\partial z^{2}}\right)-2 z \frac{\partial C}{\partial z}\right)= \\
& \quad \sum_{n, m} H_{2 m}(z \sqrt{n}) \exp -n z^{2}[4 \Gamma \dot{g}(m, n)+2(2 m+1) g(m, n)-(n-1) g(m-1, n)]
\end{aligned}
$$

as the linear-equation portion. The non-linear term in parentheses in Eq. (5.02) differs from the $D(C)=D_{0}(1+\alpha C)$ model by an ' $R$ ' factor: 


$$
\begin{aligned}
& \left\{C\left(\frac{\partial^{2} \mathrm{C}}{\partial z^{2}}\right)+R\left(\frac{\partial C}{\partial z}\right)^{2}\right\}= \\
& {[\delta(\infty>(n 1, n 2) \geq 1) \delta(\infty>(m 1, m 2) \geq 0) g(m 1, n 1) g(m 2, n 2) \times} \\
& \quad\left[\frac{1}{2}(n 1) H_{2 m 1+2}(z \sqrt{n 1}) H_{2 m 2}(z \sqrt{n 2})+\frac{1}{2}(n 2) H_{2 m 1}(z \sqrt{n 1}) H_{2 m 2+2}(z \sqrt{n 2})+\right. \\
& R(n 1)^{1 / 2}(n 2)^{1 / 2} H_{2 m 1+1}(z \sqrt{n 1}) H_{2 m 2+1}(z \sqrt{n 2}] \exp -(n 1+n 2) z^{2}
\end{aligned}
$$

For $R \geq 2, \quad\left(R C^{R-1}\right)$ is neither zero nor one, and one has the additional term:

$$
\begin{aligned}
& C^{R-1}=\sum \delta(\infty>(n 3, n 4, \ldots n *(R+1)) \geq 1) \delta(\cos (m 3, m 4, \ldots M *(R+1)) \geq 0) \times \\
& {\left[g(m 3, n 3) g(m 4, n 4) \ldots g\left(m^{*}(R+1), n^{*}(R+1)\right)\right] \exp -\left[(n 3+n 4+\ldots n *(R+1)) z^{2}\right] \times} \\
& {\left[H_{2 m 3}(z \sqrt{n 3}) H_{2 m 4}(z \sqrt{n 4}) \ldots H_{2 m *(R+1)}(z \sqrt{n *(R+1)})\right]}
\end{aligned}
$$

Recalling : $n^{*} j$ ' $\rightarrow$ ' $n_{j}$ ' and defining

$$
\xi=z \sqrt{n} ; a_{j}=[(n * j) / n]^{1 / 2}, j=(1, R+1)
$$

one next, as with the $D(C)=D_{0}(1+\alpha C)$ situation, performs the two steps:

(1). Equate coefficients of $\left(\exp -n z^{2}\right)$

(2). Multiply both sides by $\mathrm{H}_{2 \mathrm{p}}(z \sqrt{\mathrm{n}}) \mathrm{d}(\mathrm{z} \sqrt{\mathrm{n}})=\mathrm{H}_{2 \mathrm{p}}(\xi) \mathrm{d} \xi$ and integrate. This isolates a separate equation for each $g(p, n)$ :

$$
\begin{aligned}
& \pi^{1 / 2} 2^{2 p}(2 p) ![4 \dot{\Gamma} \dot{g}(p, n)+2(2 p+1) g(p, n)-(n-1) g(p-1, n)]= \\
& \gamma_{0} R n \sum g(m 1, n 1) g(m 2, n 2) \ldots g\left(m^{*}(R+1), n *(R+1)\right) \delta(n 1+n 2+\ldots+n *(R+1)=n) \times \\
& \int\left\{\frac{1}{2} a_{1}{ }^{2} H_{2 m 1+2}\left(a_{1} \xi\right) H_{2 m 2}\left(a_{2} \xi\right)+\frac{1}{2} a_{2}{ }^{2} H_{2 m 1}\left(a_{1} \xi\right) H_{2 m 2+2}\left(a_{2} \xi\right)+\right. \\
& \left.\mathrm{Ra}_{1} \mathrm{a}_{2} \mathrm{H}_{2 \mathrm{~m} 1+1}\left(\mathrm{a}_{1} \xi\right) \mathrm{H}_{2 \mathrm{~m} 2+1}\left(\mathrm{a}_{2} \xi\right)\right\} \times \\
& \mathrm{H}_{2 \mathrm{~m} 3}\left(\mathrm{a}_{3} \xi\right) \cdot \mathrm{H}_{2 \mathrm{~m} 4}\left(\mathrm{a}_{4} \xi\right) \ldots \mathrm{H}_{2 \mathrm{~m}^{*}(\mathrm{R}+1)}\left(\mathrm{a}_{\mathrm{R}+1} \xi\right) \mathrm{H}_{2 \mathrm{p}}(\xi) \exp -\left(\xi^{2}\right) \mathrm{d} \xi \quad \times \\
& \delta\left(a_{1}{ }^{2}+a_{2}{ }^{2}+\ldots+a_{R+1}^{2}=1\right) \delta(\infty>(n 1, n 2, \ldots n *(R+1)) \geq 1) \delta\left(\infty>\left(m 1, m 2, \ldots m^{*}(R+1)\right) \geq 0\right)
\end{aligned}
$$


It is easily verified that: 50

$$
\mathrm{H}_{2 q}\left(\mathrm{a}_{j} \xi\right) \exp -\left(\mathrm{a}_{j} \xi\right)^{2}=\operatorname{Limit}_{t_{j} \rightarrow 0}\left(\frac{\partial}{\partial t_{j}}\right)^{2 q} \exp -\left(t_{j}-a_{j} \xi\right)^{2} .
$$

Using that, and also using:

$$
\exp -\xi^{2} \equiv \exp -\left(\mathrm{a}_{1}{ }^{2} \xi^{2}+\mathrm{a}_{2}{ }^{2} \xi^{2}+\ldots+\mathrm{a}_{\mathrm{R}+1^{2}} \xi^{2}\right)
$$

the integral (Eq. 5.07) can be converted into the following form:

$$
\begin{aligned}
& \int\{\} \ldots \exp -\left(\xi^{2}\right) d \xi= \\
& \operatorname{timit}_{1}, t_{2, \ldots t_{R+1} \rightarrow 0} \cdot\left\{\frac{1}{2} a_{1}^{2}\left(\frac{\partial}{\partial t_{1}}\right)^{2 m 1+2}\left(\frac{\partial}{\partial t_{2}}\right)^{2 m 2}+\frac{1}{2} a_{2}^{2}\left(\frac{\partial}{\partial t_{1}}\right)^{2 m 1}\left(\frac{\partial}{\partial t_{2}}\right)^{2 m 2+2}\right. \\
& \left.+R_{1} a_{2}\left(\frac{\partial}{\partial t_{1}}\right)^{2 m 1+1}\left(\frac{\partial}{\partial t_{2}}\right)^{2 m 2+1}\right\} e^{-\left(t_{1}-a_{1} \xi\right)^{2}} e^{\left.-t_{2}-a_{2} \xi\right)^{2}} \times \\
& \left(\frac{\partial}{\partial t_{3}}\right)^{2 m 3} e^{-\left(t_{3}-a_{3} \xi\right)^{2}}\left(\frac{\partial}{\partial t_{4}}\right)^{2 m 4} e^{-\left(t_{4}-a_{4} \xi\right)^{2}} \ldots x \\
& \left(\frac{\partial}{\partial t_{R+1}}\right)^{2 m *(R+1)} e^{-\left(t_{R+1}-a_{R+1} \xi\right)^{2}} H_{2 p}(\xi) \delta\left(a_{1}{ }^{2}+a_{2}{ }^{2}+\ldots+a_{R+1}^{2}=1\right) d \xi \text {. }
\end{aligned}
$$

The great advantage of this formalism is that one can factor out most of the terms within the $\{\cdot\}$. Specifically:

\{\}$=\left\{\frac{1}{2} a_{1}{ }^{2}\left(\frac{\partial}{\partial t_{1}}\right)^{2}+\frac{1}{2} a_{2}{ }^{2}\left(\frac{\partial}{\partial t_{2}}\right)^{2}+R a_{1} a_{2}\left(\frac{\partial}{\partial t} \frac{\partial}{\partial t_{2}}\right)\right\}\left(\frac{\partial}{\partial t_{1}}\right)^{2 m 1}\left(\frac{\partial}{\partial t_{2}}\right)^{2 m 2}$

This gives:

$$
\begin{aligned}
& \int\{\} \because \exp -\left(\xi^{2}\right) \mathrm{d} \xi= \\
& { }_{t_{1}, \ldots t_{R+1 \rightarrow 0}}^{\text {Limit }} \int\left\{\frac{1}{2} a_{1}{ }^{2}\left(\frac{\partial}{\partial t_{1}}\right)^{2}+\frac{1}{2} a_{2}{ }^{2}\left(\frac{\partial}{\partial t_{2}}\right)^{2}+R a_{1} a_{2}\left(\frac{\partial}{\partial t_{1}} \frac{\partial}{\partial t_{2}}\right)\right\} \times \\
& \prod_{j=1}^{R+1}\left(\frac{\partial}{\partial t_{j}}\right)^{2 m^{*} j} \exp -\left(\sum_{j=1}^{R+1}\left[t_{j}-a_{j} \xi\right]^{2}\right) H_{2 p}(\xi) d \xi \text {. }
\end{aligned}
$$

The term now within the braces: \{\} is symmetric upon interchange of 
$\left(a_{1}, t_{1}\right)$ with $\left(a_{2}, t_{2}\right)$. Since this appears within a large sum (over indices $\left[m 1, m 2 \ldots m^{*}(R+1)\right]$ and over indices $[n 1, n 2, \ldots n *(R+1])$, it is always possible to re-label.

In other words, in the integral (Eq. (5.12)) the $\left(a_{1}, a_{2}\right)$ (along with the associated $\left(t_{1}, t_{2}\right)$ ) does not occupy a special position compared to $\left(a_{3}, a_{4} \ldots\right)$ (and its associated $\left(t_{3}, t_{4} \ldots\right)$ ), because labels could be switched so that $\left(a_{4}, a_{5}\right)$, or any other pair $\left(a_{k 1}, a_{k 2}\right)$, could occupy that 'special' position. Thus, the roles of $\left(a_{1}, a_{2}, \ldots\right)$, can be symmeterized. Since $R(R+1) / 2$ distinct \{\} -terms can be formed with the pairs $\left[\left(a_{k 1}, a_{k 2}\right), k 1 \neq k 2\right]$, one can replace the integral (Eq. 5.12) with the following different (symmeterized) integral:

$$
\begin{aligned}
& \operatorname{timit}_{1}, \ldots t_{R+1 \rightarrow 0} \int(2 / R(R+1)) \sum_{i \neq j}\left\{\frac{1}{2} a_{i}^{2}\left(\frac{\partial}{\partial t}\right)_{i}^{2}+\frac{1}{2} a_{j}^{2}\left(\frac{\partial}{\partial t}\right)_{j}^{2}+R a_{i} a_{j}\left(\frac{\partial}{\partial t} \frac{\partial}{\partial t}\right)\right\} \\
& \times H_{2 p}(\xi) \prod_{k 1=1}^{R+1}\left(\frac{\partial}{\partial t} \cdot\right)^{2(m * k 1)} \exp -\left(\sum_{k=1}^{R+1}\left[t_{k}-a_{k} \xi\right]^{2}\right) d \xi
\end{aligned}
$$

where there are exactly $\frac{1}{2} R(R+1)$ terms in the sum:

$$
\sum_{i \neq j}\{\} \equiv \sum_{i=1}^{R+1} \sum_{j=1}^{R+1} \delta(i \neq j)\{\} .
$$

The following algebraic tautology:

$$
\sum_{i=1}^{R+1} \sum_{j=1}^{R+1} \delta(i \neq j)\left\{\frac{1}{2}\left(q_{i}{ }^{2}+q_{j}{ }^{2}\right)+R q_{i} q_{j}\right\} \equiv \frac{R}{2}\left[\sum_{i=1}^{R+1} q_{j}\right]^{2}
$$

where each distinct $\left(\frac{1}{2} q_{i}{ }^{2}\right)$ term appears exactly ' $R$ ' times, then gives:

$$
\begin{gathered}
(2 / R(R+1)) \sum_{i \neq j}\left\{\frac{1}{2} a_{i}^{2}\left(\frac{\partial}{\partial t}\right)_{i}^{2}+\frac{1}{2} a_{j}^{2}\left(\frac{\partial}{\partial t}\right)_{j}^{2}+R a_{i} a_{j}\left(\frac{\partial}{\partial t_{i}} \frac{\partial}{\partial t_{j}}\right)\right\}= \\
(1 /(1+R))\left[\sum_{i=1}^{R+1} a_{i} \frac{\partial}{\partial t_{i}}\right]^{2}
\end{gathered}
$$


Thus, Eq. 5.07 becomes:

$$
\begin{aligned}
& \pi^{1 / 2} 2^{2 p}(2 p) ![4 \Gamma \dot{g}(p, n)+2(2 p+1) g(p, n)-(n-1) g(p-1, n)]=\quad(5.16) \\
& \frac{R n \gamma}{R+1} 0 g(m 1, n 1) g(m 2, n 2) \ldots g\left(m^{*}(R+1), n^{*}(R+1)\right) \delta\left(n 1+n 2+\ldots n^{*}(R+1)=n\right) x \\
& \text { Limit } \\
& t_{1} \ldots t_{R+1} \rightarrow 0 \int\left[\sum_{i=1}^{R+1} a_{i} \frac{\partial}{\partial t}\right]_{i}^{2} H_{2 p}(\xi) \prod_{j=1}^{R+1}\left(\frac{\partial}{\partial t}\right)_{j}^{2 m^{*} j} \exp -\left(\sum_{k=1}^{R+1}\left[t_{k}-a_{k} \xi\right]^{2}\right) d \xi \times \\
& \delta\left(a_{1}{ }^{2}+a_{2}{ }^{2}+\ldots+a_{R+1}{ }^{2}=1\right) \delta\left(\infty>\left(n 1, n 2, \ldots n^{*}(R+1)\right) \geq 1\right) \delta\left(\infty>\left(m 1, \ldots m^{*}(R+1)\right) \geq 0\right)
\end{aligned}
$$

Defining:

$$
\begin{aligned}
& \theta\left(m 1, m 2, \ldots m^{*}(R+1), n 1, n 2, \ldots n^{*}(R+1) ; p, n\right)= \\
& \left(\pi^{1 / 2}(2 p) ! 2^{2 p}\right)^{-1} \prod_{q=1}^{R+1} \delta\left(\left(n^{*} q\right) a_{q}^{2}=1\right) \quad \delta\left(a_{1}{ }^{2}+a_{2}{ }^{2}+a_{3}{ }^{2}+\ldots a_{R+1}^{2}=1\right) \times \\
& \operatorname{Limit}_{1}, \ldots t_{R+1 \rightarrow 0} \int_{i=1}^{R+1}\left[\sum_{i} a_{i} \frac{\partial}{\partial t}\right]^{2} H_{2 p}(\xi) \prod_{j=1}^{R+1}\left(\frac{\partial}{\partial t}\right)_{j}^{2 m^{*} j} \exp -\left(\sum_{k=1}^{R+1}\left[t_{k}-a_{k} \xi\right]^{2}\right) d \xi
\end{aligned}
$$

gives finally:

$[4 \Gamma \dot{g}(p, n)+2(2 p+1) g(p, n)-(n-1) g(p-1, n)]=$

$n \gamma_{0} R(R+1)^{-1}\left[g(m 1, n 1) g(m 2, n 2) \ldots g\left(m^{*}(R+1), n^{*}(R+1)\right) \delta\left(n 1+n 2+\ldots n^{*}(R+1)=n\right) \quad x\right.$

$\Theta\left(m 1, m 2, \ldots m^{*}(R+1), n 1, n 2, \ldots n^{*}(R+1) ; p, n\right) \quad x$

$\delta(\infty>(n 1, n 2, \ldots n *(R+1)) \geq 1) \quad \delta\left(\infty \geq\left(m 1, m 2, \ldots m^{*}(R+1)\right) \geq 0\right)$

This is a direct analog to the $D(C)=D_{0}(1+\alpha C)$ problem, except it is for the $D(C)=D_{0}\left(1+\gamma_{0} R C^{R}\right)$ problem. The final (symmeterized) form

(Eq. 5.17) is valid for all non-negative integers ' $R$ ', and $\theta(\ldots)$ exactly matches the previous definition of Chapter IV for the $(R=1)$ case. The extension to $D(C)=D_{0}\left(1+\alpha C+\beta C^{2}+\gamma C^{3}+\ldots\right)$ is obvious. Since the only effect of the $D(C)=D_{0}\left(1+\gamma R C^{R}\right)$ problem is to alter the righthand-side, and it only involves more sums and a bigger $\theta(\ldots)$ function, 
one has, for $D(C)$ being a polynomial in ' $C$ ', exactly the form given previously $(E q \cdot 4.101)$.

And, as with the $D(C)=D_{0}(1+\alpha C)$ function, to automatically preserve normalization, one needs (for all $R=$ (integer) ):

$$
\theta\left(m 1, m 2, \ldots m^{*}(R+1), n 1, n 2 ; \ldots n *(R+1) ; p=0, n\right)=0
$$

We note that as ' $R$ ' gets larger and larger, this represents a larger and larger integral which must vanish. In Appendix V, that (Eq. 5.01) is true will be explicitly demonstrated for all $R=$ (positive integers). A formalism to do the integral embodied in $\theta(\ldots)$ will also be given there, as well as the explicit calculation for $(R=1)$ and $(R=2)$. The rest of the structure developed for the $D(C)=D_{0}(1+\alpha C)$ problem carries over directly to the $D(C)=D_{0}\left(1+\alpha C+B C^{2} \ldots\right)$ situation:

The concentration dependent diffusivity which is a small polynomial function of ' $C$ ' is thus solved. Again, it is analytic, conserves total mass: $\int \mathrm{Cdx}=1$, and allows an arbitrary function for an initial state.

We proceed in the next chapter, to construct the actual functions $g(m, n)$ for the $D(C)=D_{0}\left(1+\alpha C+B C^{2}\right)$ problem with

$$
C=\phi_{0}(z, \Gamma)+\alpha \dot{\phi}_{1}(z, \Gamma)+\alpha^{2} \phi_{2}(z, \Gamma)+\beta \phi_{3}(z, \Gamma) \ldots
$$

i.e., this will be a solution up to $\operatorname{order}-\left(\alpha^{2}, \beta\right)$; one which calculates $g(m, n)$ explicitly as a function of ' $\Gamma$ ' for $n=(1,2,3)$. 
CHAPTER 6

\section{EXPLICIT CALCULATION OF THE \\ $g(p, n)$ FUNCTIONS}

\section{The Calculation Setup}

Using the (expanded) "fundamental" equation for the $D(C)=$ $D_{0}\left(1+\alpha C+B C^{2}+\ldots\right)$ model (Eq. 4.101) and the transformation:

$$
g(p, n)=[.25(n-1)+\delta(n=1)]^{p} \Gamma^{-(p+1 / 2)} e(p, n)
$$

then gives:

$$
\begin{aligned}
& {[\dot{e}(p, n)-(1-\delta(n=1)) e(p-1, n)]=.25\{4 /[(n-1)+4 \delta(n=1)]\}^{p} \Gamma^{p-1 / 2} \times} \\
& \left((\alpha \mathrm{n} / 2) \sum_{\mathrm{n} 1, \mathrm{n} 2 \mathrm{~m} 1, \mathrm{~m} 2} \delta(\mathrm{n} 1+\mathrm{n} 2=\mathrm{n})[.25(\mathrm{n} 1-1)+\delta(\mathrm{n} 1=1)]^{\mathrm{m} 1}[.25(\mathrm{n} 2-1)+\delta(\mathrm{n} 2=1)]^{\mathrm{m} 2} \times\right.
\end{aligned}
$$

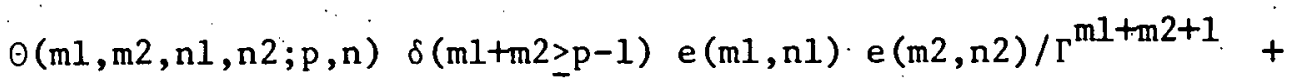

$$
\begin{aligned}
& (B n / 3) \sum_{n 1, n 2, n 3 . m 1, m 2, m 3} \delta(n 1+n 2+n 3=n) . \times \\
& {[.25(n 1-1)+\delta(n 1=1)]^{m 1}[.25(n 2-1)+\delta(n 2=1)]^{m 2} \times} \\
& {[.25(\mathrm{n} 3-1)+\delta(\mathrm{n} 3=1)]^{\mathrm{m} 3} \Theta(\mathrm{m} 1, \mathrm{~m} 2, \mathrm{~m} 3, \mathrm{n} 1, \mathrm{n} 2, \mathrm{n} 3 ; \mathrm{p}, \mathrm{n}) \delta(\mathrm{ml}+\mathrm{m} 2+\mathrm{m} 3 \geq \mathrm{p}-1) \times} \\
& \left.[e(m 1, n 1) e(m 2, n 2) e(m 3, n 3)] / \Gamma^{m 1+m 2+m 3+3 / 2}+\ldots\right)
\end{aligned}
$$

where the implicit limits of each $\left(n^{*} j\right)-$ sum are $\delta\left(\infty>\left(n^{*} j\right) \geq 1\right)$ and the implicit limits of each $\left(m^{*} j\right)$-sum are $\delta\left(\infty>\left(m^{*} j\right) \geq 0\right)$. Whe also use the limits :

$$
\operatorname{Limit}_{\Gamma \rightarrow \Gamma_{0}} e(p, n)=B_{p} \delta(n=1)=(0 \text { if } n \neq 1)
$$




$$
(\text { for all } \Gamma) \quad e(0, n)=B_{0} \delta(n=1)=(0 \text { if } n \neq 1)
$$

which, of course, are based on $\theta\left(m 1, m 2 \ldots m^{*}(R+1), n 1, n 2, \ldots n *(R+1) ; p=0, n\right)=0$. The additional constant $\delta\left(m 1+m 2+\ldots+m^{*}(R+1) \geq p-1\right)$ (see Appendix $V$ ) embodies the fact that, if not satisfied, the function $\theta\left(m 1, m 2, \ldots m^{*}(R+1), n 1, n 2 \ldots\right.$ $n *(R+1) ; p, n)=0$.

A calculation using $D(C)=D_{0}\left(1+\alpha C+B C^{2}\right)$ involves taking only the $(\alpha, \beta)$ terms of $(E q .6 .02)$. A calculation of ' $C$ ' to order $\left(\alpha^{2}, \beta\right)$ is equivalent to solving for $e(p ; n)$ for $n=(1,2,3)$.

As given before, the $(n=1)$ case is simple and $e(p, 1)$ as a function of $\Gamma$ is:

$$
\mathrm{e}(\mathrm{p}, 1)=\text { constant }=\mathrm{B}_{\mathrm{p}}
$$

We next calculate the $n=2$ and $n=3$ cases. The $\alpha$-term has a portion:

$$
\begin{aligned}
& .25\{4 /[(n-1)+4 \delta(n=1)]\}^{p}(\alpha n / 2) \delta(n 1+n 2=n) \times \\
& {[.25(n 1-1)+\delta(n l=1)]^{m l} \cdot[.25(n 2-1)+\delta(n 2=1)]^{m 2}}
\end{aligned}
$$

For the $(n=2)$ case, there is only one contribution: $(n 1=1, n 2=1)$ and this reduces to:

$$
(\alpha / 4) \cdot 4^{p}
$$

For the $(n=3)$ case, there are two equal contributions: ( $n 1=1, n 2=2)$ and $(n 1=2, n 2=1)$. Together, they give:

$$
(3 \dot{\alpha} / 4) 2^{\mathrm{P}}\left(4^{-\mathrm{m} 2}\right) \text {. }
$$

Also, for the $(n=3)$ case, the $\beta$-term:

$$
\begin{aligned}
& .25\{4 /[(n-1)+4 \delta(n=1)]\}^{p}(\beta n / 3) \delta(n 1+n 2+n 3=n) \times \\
& {[.25(n 1-1)+\delta(n 1=1)]^{m 1}[.25(n 2-1)+\delta(n 2=1)]^{m 2}[.25(n 3-1)+\delta(n 3=1)]^{m 3}}
\end{aligned}
$$


has a single contribution $(\mathrm{n} 1=1, \mathrm{n} 2=\mathrm{n} 3=1)$ which gives:

$$
(B / 4) 2^{p}
$$

The e(p,n) equations become (for $n=2$ and $n=3$ ):

$$
\begin{aligned}
& {[\dot{e}(p ; 2)-e(p-1,2)]=} \\
& (\alpha / 4) 4^{p} \sum_{m 1 ; m 2} \theta(m 1, m 2,1,1 ; p, 2) \delta(m 1+m 2 \geq p-1) \times \\
& e(m 1,1) \text { e }(m 2 ; 1) / \Gamma^{m 1+m 2-p+3 / 2} \\
& {[\dot{e}(p, 3)-e(p-1,3)]=} \\
& (3 \dot{\alpha} / 4) 2^{\mathrm{p}} \sum_{\mathrm{m} 1, \mathrm{~m} 2} \odot(\mathrm{ml}, \mathrm{m} 2,1,2 ; \mathrm{p}, 3) \delta(\mathrm{ml}+\mathrm{m} 2 \geq \mathrm{p}-1) \times \\
& e(m 1,1) e(m 2,2) 4^{-m 2} / \Gamma^{m 1+m 2-p+3 / 2}+ \\
& (B / 4) 2^{\mathrm{p}} \sum_{\mathrm{m} 1, \mathrm{~m} 2, \mathrm{~m} 3} \Theta(\mathrm{ml}, \mathrm{m} 2, \mathrm{~m} 3,1,1,1 ; \mathrm{p}, 3) \delta(\mathrm{m} 1+\mathrm{m} 2+\mathrm{m} 3 \geq \mathrm{p}-1) \times \\
& e(m 1,1) e(m 2,1) \cdot e(m 3,1) / \Gamma^{m 1+m 2+m 3-p+2} \text {. }
\end{aligned}
$$

Since $e(m, 1)=B_{m}$ which are coefficients determined from the initial state:

$$
c\left(x, t_{R}\right)=\sum_{m=0}^{N 1} B_{m} H_{2 m}\left(x / x_{R}\right) \exp -\left(x / x_{R}\right)^{2}
$$

there being only $(\mathrm{N} 1+1)$ polynomials in a given fit; the sums on $(\mathrm{m} 1, \mathrm{~m} 2)$ in the $e(p, 2)$-equation are finite sums, limited by the data fit one chooses. The collection $e(p, 2)$, though, has in principle, an infinite number of terms. However, given a finite (N1), one expects that the most important. $e(p, 2)$ terms come from the $p$-range near $p=1$ (recalling $e(0,2) \equiv 0)$. Thus, we introduce a cut-off on the number of $e(p, 2)$ terms that are calculated:

$$
\left[e(p, 2) ; p=\left(0, N^{\prime}\right)\right]=(N A+1) \text {-terms }
$$


Similarly, for the $e(p, 3)$ functions, the cut-off is introduced:

$$
\left[e(p ; 3) ; p=\left(0,{ }^{\prime} N B^{\prime}\right)\right]=(N B+1) \text {-terms. }
$$

This is equivalent to the approximation:

$$
\begin{aligned}
C(z, \Gamma) \doteq & {\left[\delta(N 1 \geq p \geq 0) g(p, 1) H_{2 p}(z) \exp -z^{2}+\right.} \\
& {\left[\delta(N A \geq p \geq 0) g(p, 2) H_{2 p}(z \sqrt{2}) \exp -2 z^{2}+\right.} \\
\therefore & {\left[\delta(N B \geq p \geq 0) g(p, 3) H_{2 p}(z \sqrt{3}) \exp -3 z^{2} .\right.}
\end{aligned}
$$

In the next sections, which proceed with this explicit calculation of the $n=2$ and $n=3$ cases for this model, extensive use will be made of the sum permutation formalism introduced in (Sec. III; Chapter 4).

$$
* * *
$$

\section{The Order $-(\alpha),(n=2)$ Case}

With $e(m, 1)=B_{m}$, the $n=2$ equation becomes:

$$
\begin{gathered}
{[\dot{\mathrm{e}}(\mathrm{p}, 2)-\mathrm{e}(\mathrm{p}-1,2)]=(\alpha / 4) 4^{\mathrm{p}}[\delta(\mathrm{N} 1 \geq \mathrm{p} 1 \geq 0) \delta(\mathrm{N} 1 \geq \mathrm{p} 2 \geq 0)} \\
\delta(\mathrm{p} 1+\mathrm{p} 2 \geq \mathrm{p}-1) \dot{\theta}(\mathrm{p} 1, \mathrm{p} 2,1,1 ; \mathrm{p}, 2) \quad \mathrm{B}_{\mathrm{p} 1} \mathrm{~B}_{\mathrm{p} 2} / \Gamma^{\mathrm{p} 1+\mathrm{p} 2-\mathrm{p}+3 / 2}
\end{gathered}
$$

Defining $\mathrm{pa} \equiv \mathrm{p} 1+\mathrm{p} 2$ and using additive combination to replace ' $\mathrm{p} 2$ ':

$$
\begin{aligned}
& {[\dot{e}(p, 2)-e(p-1,2)]=(\alpha / 4) 4^{p}\left[\delta(2 N 1 \geq p a \geq 0):^{x}\right.} \\
& \delta(p a \geq p-1) R 2(p, p a) / \Gamma^{p a-p+3 / 2} \\
& R 2(p, p a)=[\delta(\min [p a, N 1] \geq p 1 \geq \max [0, p a-N 1]) \times \\
& \theta(p 1, p a-p 1,1,1 ; p, 2) B_{p 1} B(p a-p 1)
\end{aligned}
$$

Hotice the entire array 'R2' are specified constants. . Defining $\mathrm{f}^{-1}(\mathrm{p}, \mathrm{n}=2 ; \Gamma)$. as:

$$
\tilde{\mathrm{f}}^{-1}(\mathrm{p}, 2 ; \Gamma)=4^{\mathrm{p}-1} \sum \delta(2 \mathrm{~N} 1 \geq \mathrm{pa} \geq \max [0, \mathrm{p}-1]) R 2(\mathrm{p}, \mathrm{pa}) / \Gamma^{\mathrm{pa}-\mathrm{p}+3 / 2}
$$


gives:

$$
\dot{e}(p, 2)-e(p-1,2)=\alpha \tilde{f}^{-1}(p, 2 ; \Gamma)
$$

which has the solution:

$$
e(p, 2)=\alpha \sum_{q=0}^{p-1} \tilde{f}^{q}(p-q, 2 ; \Gamma)
$$

First, constructing $\tilde{\mathrm{f}} \mathrm{q}(p, 2 ; \Gamma)$ :

$$
\begin{aligned}
& \mathrm{f}^{\mathrm{q}}(\mathrm{p}, 2 ; \Gamma)=4^{\mathrm{p}-1} \sum \delta(2 \mathrm{~N} 1 \geq \mathrm{pa}-\max [0, \mathrm{p}-1]) \mathrm{R} 2(\mathrm{p}, \mathrm{pa}) \times \\
& \int \cdots \int\left(1 / \Gamma^{\mathrm{pa}-\mathrm{p}+3 / 2}\right)(\mathrm{d} \Gamma)^{\mathrm{q}+1}= \\
& 4^{\mathrm{p}-1}\left[\delta(2 \mathrm{~N} 1 \geq \mathrm{pa} \geq \max [0, \mathrm{p}-1]) \mathrm{R} 2(\mathrm{p}, \mathrm{pa}) \Phi\left(\mathrm{p}-\mathrm{pa}-3 / 2,0, \mathrm{q} ; \Gamma, \Gamma_{0}\right)\right.
\end{aligned}
$$

immediately e $(p, 2)$ can be found:

$$
\begin{aligned}
& e(p, 2)=\alpha \sum_{q=0}^{p-1} 4^{p-q-1} \sum \delta(2 N 1 \geq p a>\max [0, p-q-1]) R 2(p-q, p a) \times \\
& \Phi\left(p-q-p a-3 / 2,0, q ; \Gamma, \Gamma_{0}\right) .
\end{aligned}
$$

Using the expression for $\Phi\left(\nu, 0, q ; \Gamma, \Gamma_{0}\right)$ for $\nu=$ (half-integer):

$$
\Phi\left(v, 0, q ; \Gamma, \Gamma_{0}\right)=(1 / q !) \sum_{m=0}^{q}\left({ }_{m}^{q}\right)(-1)^{m} \Gamma^{q-m}\left[\Gamma^{m+v+1}-\Gamma_{0}^{m+v+1}\right] /(m+v+1)(6.19)
$$

and summing the m-sum in reverse order, one can get for $v=p-q-p a-3 / 2$ :

$$
\begin{gathered}
\Phi\left(\nu, 0, q ; \Gamma, \Gamma_{0}\right)=(1 / q !) \Gamma^{p-p a-1 / 2} \sum_{m=0}^{q}\left({ }_{m}^{q}\right)(-1)^{q-m} /(p-m-p a-1 / 2) \\
-(1 / q !) \Gamma_{0}^{p-p a-1 / 2} \sum_{m=0}^{q}\left({ }_{m}^{q}\right)(-1)^{q-m}\left(\Gamma / \Gamma_{0}\right)^{m} /(p-m-p a-1 / 2)
\end{gathered}
$$

Substituting this into $e(p, 2)$ and doing a permutation of the (q,pa) sums in one term, and a $(\mathrm{q}, \mathrm{m})$ permutation in the other; one can get:

$$
e(p, 2)=\alpha \sum_{p a=0}^{2 N 1} \Gamma^{p-p a-1 / 2} s 2 A(p, p a)-\alpha \sum_{m=0}^{p-1}\left(\Gamma / \Gamma_{o}\right)^{m} s 2 B(p, m)
$$


The definition of the arrays 'S2A' and 'S2B' are:

$$
\begin{aligned}
& \mathrm{s} 2 \mathrm{~A}(\mathrm{p}, \mathrm{pa})=\left[\delta(\mathrm{p}-1 \geq \mathrm{m} \geq 0)(\mathrm{p}-\mathrm{pa}-\mathrm{m}-1 / 2)^{-1} / \mathrm{m} ! \times\right. \\
& \sum \delta(\mathrm{p}-1 \geq \mathrm{q} \geq \max [\mathrm{m}, \mathrm{p}-\mathrm{pa}-1]) 4^{\mathrm{p}-\mathrm{q}-1} \cdot \mathrm{R} 2(\mathrm{p}-\mathrm{q}, \mathrm{pa})(-1)^{\mathrm{q}-\mathrm{m}} /(\mathrm{q}-\mathrm{m}) ! \\
& S 2 B(p, m)=\sum \delta(2 N 1 \geq p a \geq 0) \Gamma_{0}^{p-p a-1 / 2}(p-p a-m-1 / 2)^{-1 / m !} \times \\
& \sum \delta(\mathrm{p}-1 \geq \mathrm{q} \geq \max [\mathrm{m}, \mathrm{p}-\mathrm{pa-1}]) 4^{\mathrm{p}-\mathrm{q}-1} \mathrm{R} 2(\mathrm{p}-\mathrm{q}, \mathrm{pa})(-1)^{\mathrm{q}-\mathrm{m}} \cdot /(\mathrm{q}-\mathrm{m}) !
\end{aligned}
$$

Notice the (q)-sum portions are identical and, as with 'R2', the 'S2A' and 'S2B' arrays are entirely specified constants. Finally converting to $g(p, 2):$

$$
\begin{gathered}
g(p, 2)=(1 / 4)^{p_{\Gamma}^{-(p+1 / 2)}} e(p, 2) \\
\therefore \\
4^{p} g(p, 2) / \alpha=\sum_{r=0}^{2 N 1} \Gamma^{-r-1} \operatorname{se}(p, r)-\sum_{r=0}^{p-1} \Gamma^{-r-3 / 2}\left(\Gamma_{0}^{r-p+1}\right) s 2 B(p, p-r-1) .
\end{gathered}
$$

\section{The Order $-\left(\alpha^{2}, \beta\right),(n=3)$ Case}

The $(n=3)$ equation is (for $p \geq 1$, recalling that $e(0,3)=0)$ :

$$
[\dot{e}(p, 3)-\dot{e}(p-1,3)]=
$$

$(3 \alpha / 4) 2^{\mathrm{P}}[\delta(\mathrm{N} 1 \geq \mathrm{p} 1 \geq 0) \delta(\mathrm{NA} \geq \mathrm{p} 2 \geq 0) \delta(\mathrm{p} 1+\mathrm{p} 2 \geq \mathrm{p}-1) \quad \times$

$\Theta(p 1, p 2,1,2 ; p, 3) 4^{-p 2} \cdot e(p 1,1) e(p 2,2) / \Gamma^{p 1+p 2-p+3 / 2}+$

$(B / 4) 2^{\mathrm{P}}[\delta(\mathrm{N} 1 \geq \mathrm{p} 1 \geq 0) \delta(\mathrm{N} 1 \geq \mathrm{p} 2 \geq 0) \delta(\mathrm{N} 1 \geq \mathrm{p} 3 \geq 0) \delta(\mathrm{p} 1+\mathrm{p} 2+\mathrm{p} 3 \geq \mathrm{p}-1) \cdot x$

$\odot(p 1, p 2, p 3,1,1,1 ; p, 3)$ e $(p 1,1) e(p 2,1) e(p 3,1) / \Gamma^{p 1+p 2+p 3-p+2}$

Substituting directly for $e(p 2,2)$, recalling that $e(0,2)=0$, so that 


$$
\begin{aligned}
& \mathrm{p} 2(\min )=1 ; \text { and using } \mathrm{e}(\mathrm{m} 1)=\mathrm{B}_{\mathrm{m}} \text {, one gets: } \\
& {[\dot{\mathrm{e}}(\mathrm{p}, 3)-\mathrm{e}(\mathrm{p}-1,3)]=\{\text { Term } 1\}+\{\text { Term } 2\}+\{\text { Term } 3\}}
\end{aligned}
$$

Examining the first term:

$$
\begin{aligned}
& \{\text { Term } 1\}=\left(3 \alpha^{2} / 4\right) 2^{\mathrm{p}}[\delta(\mathrm{N} 1 \geq \mathrm{p} 1>0) \delta(\mathrm{NA} \geq \mathrm{p} 2 \geq 1) \delta(\mathrm{p} 1+\mathrm{p} 2 \geq \mathrm{p}-1) \times \\
& \theta(\mathrm{p} 1, \mathrm{p} 2,1,2 ; \mathrm{p}, 3) 4^{-\mathrm{p} 2}\left(\mathrm{~B}_{\mathrm{p} 1} / \Gamma^{\mathrm{p} 1+\mathrm{p} 2-\mathrm{p}+3 / 2}\right) \times \\
& \sum \delta(2 \mathrm{~N} 1 \geq \mathrm{pa} \geq 0) \Gamma^{\mathrm{p} 2-\mathrm{pa}-1 / 2} \mathrm{~s} 2 \mathrm{~A}(\mathrm{p} 2, \mathrm{pa})
\end{aligned}
$$

and defining: 'ps. $\equiv \mathrm{p} 1+\mathrm{pa}$ and replacing ' $\mathrm{pl}$ ', one gets (where ' $\mathrm{K}$ ' is used as an arbitrary array label):

$$
\begin{aligned}
& \sum \delta(\mathrm{N} 1 \geq \mathrm{pl} \geq 0) \delta(2 \mathrm{~N} 1 \geq \mathrm{pa} \geq 0) \mathrm{K}(\mathrm{pl}, \mathrm{pa})= \\
& \sum \delta(3 \mathrm{~N} 1 \geq \mathrm{ps} \geq 0) \delta(\min [2 \mathrm{~N} 1, \mathrm{ps}] \geq \mathrm{pa} \geq \max [0, \mathrm{ps}-\mathrm{N} 1]) \mathrm{K}(\mathrm{ps}-\mathrm{pa}, \mathrm{pa})
\end{aligned}
$$

and this also gives:

$$
\delta(N A \geq p 2 \geq 1) \cdot \delta(p 1+p 2 \geq p-1)=\delta(N A \geq p 2 \geq \max [1, p+p a-p s-1])
$$

Thus, $\{$ Term 1$\}$ is transformed into:

$$
\begin{aligned}
& \{\operatorname{Term} 1\}=+\left(3 \alpha^{2} / 4\right) 2^{\mathrm{p}}\left[\delta(3 \mathrm{~N} 1 \geq \mathrm{ps} \geq 0) \mathrm{RA}(\mathrm{p}, \mathrm{ps}) / \Gamma^{\mathrm{ps}-\mathrm{p}+2}\right. \\
& \operatorname{RA}(\mathrm{p}, \mathrm{ps})=\sum \delta(\min [2 \mathrm{~N} 1, \mathrm{ps}] \geq \mathrm{pa} \geq \max [0, \mathrm{ps}-\mathrm{N} 1]) \mathrm{B}(\mathrm{ps}-\mathrm{pa}) \times \\
& \sum \delta(\mathrm{NA} \geq \mathrm{p} 2 \geq \max [1, \mathrm{p}+\mathrm{pa}-\mathrm{ps}-1]) 4^{-\mathrm{p} 2} \odot(\mathrm{ps}-\mathrm{pa}, \mathrm{p} 2,1,2 ; \mathrm{p}, 3) \mathrm{s} 2 A(\mathrm{p} 2, \mathrm{pa}) .
\end{aligned}
$$

Another term in the $e(p, 3)$ equation is:

$$
\begin{aligned}
& \{\text { Term } 2\}=-\left(3 \alpha^{2} / 4\right) 2^{\mathrm{p}}[\delta(\mathrm{N} 1 \geq \mathrm{p} 1 \geq 0) \delta(\mathrm{NA} \geq \mathrm{p} 2 \geq 1) \delta(\mathrm{p} 1+\mathrm{p} 2 \geq \mathrm{p}-1) \times \\
& O(\mathrm{p} 1, \mathrm{p} 2,1 ; 2 ; \mathrm{p}, 3) 4^{-\mathrm{p} 2}\left(\mathrm{~B}_{\mathrm{p} 1} / \Gamma^{\mathrm{p} 1+\mathrm{p} 2-\mathrm{p}+3 / 2}\right) \times \\
& \sum \delta(\mathrm{p} 2-1>\mathrm{m} \geq 0) \mathrm{S} 2 \mathrm{~B}(\mathrm{p} 2, \mathrm{~m})\left(\Gamma / \Gamma_{0}\right)^{\mathrm{m}}
\end{aligned}
$$


Using:

$$
\begin{aligned}
& \delta(N A \geq \mathrm{p} 2 \geq 1) \quad \delta(\mathrm{p} 2-1 \geq \mathrm{m} \geq 0) \quad \delta(\mathrm{p} 1+\mathrm{p} 2 \geq \mathrm{p}-1)= \\
& \delta(N A-1 \geq \mathrm{m} \geq 0) \quad \delta(\mathrm{NA} \geq \mathrm{p} 2 \geq \max [\mathrm{m}+1, \mathrm{p}-\mathrm{p} 1-1])
\end{aligned}
$$

and defining $p s \equiv p 1+m$, and replacing ' $m$ ', one gets:

$$
\begin{aligned}
& {[\delta(\mathrm{NA}-1 \geq \mathrm{m} \geq 0) \delta(\mathrm{N} 1 \geq \mathrm{p} 1 \geq 0) \mathrm{K}(\mathrm{m}, \mathrm{p} 1)=} \\
& {[\delta(\mathrm{N} 1+\mathrm{NA}-1 \geq \mathrm{ps} \geq 0) \delta(\min [\mathrm{N} 1, \mathrm{ps}] \geq \mathrm{p} 1 \geq \max [0, \mathrm{ps}-\mathrm{NA}+1])}
\end{aligned}
$$

and this further alters the ' $\mathrm{p} 2$ '-Kronecker's delta to be:

$$
\delta(\mathrm{NA} \geq \mathrm{p} 2 \geq \max [\mathrm{ps}+1, \mathrm{p}-1]-\mathrm{p} 1) .
$$

Thus, \{Term 2\}. 1s transformed into:

$$
\begin{aligned}
& \{\operatorname{Term} 2\}=-\left(3 \alpha^{2} / 4\right) 2^{\mathrm{P}}\left[\delta(\mathrm{N} 1+\mathrm{NA}-1 \geq \mathrm{ps} \geq 0) \mathrm{RB}(\mathrm{p}, \mathrm{ps}) / \Gamma^{\mathrm{ps}-\mathrm{p}+5 / 2}\right. \\
& \mathrm{RB}(\mathrm{p}, \mathrm{ps})=\left[\delta(\min [\mathrm{N} 1, \mathrm{ps}] \geq \mathrm{p} 1 \geq \max [0, \mathrm{ps}-\mathrm{NA}+1]) \mathrm{B}_{\mathrm{p} 1} \times\right. \\
& {[\delta(\mathrm{NA} \geq \mathrm{p} 2>\max [\mathrm{ps}+1, \mathrm{p}-1]-\mathrm{p} 1) \ominus(\mathrm{p} 1, \mathrm{p} 2,1,2 ; \mathrm{p}, 3) \times} \\
& 4^{-\mathrm{p} 2} \Gamma_{0}^{-(\mathrm{p} 1+\mathrm{p} 2-\mathrm{ps}-1)} \mathrm{s} 2 \mathrm{~B}(\mathrm{p} 2, \mathrm{p} 1+\mathrm{p} 2-\mathrm{ps}-1) .
\end{aligned}
$$

The third term in the $e(p, 3)$. equation is:

$$
\begin{aligned}
& \{\operatorname{Term} 3\}=+(B / 4) 2^{p} \cdot[\delta(N 1 \geq p 1 \geq 0) \delta(N 1 \geq p 2 \geq 0) \delta(N 1 \geq p 3 \geq 0) \times \\
& \delta(p 1+p 2+p 3 \geq p-1) \odot(p 1, p 2, p 3,1,1,1 ; p, 3) \times \\
& { }^{B}{ }_{p 1}{ }^{B}{ }_{p 2}{ }^{B}{ }_{p} / \Gamma(p 1+p 2+p 3-p+2)
\end{aligned}
$$

Defining: $p a \doteq \mathrm{p} 2+\mathrm{p} 3$ and replacing 'p3' gives:

$$
\begin{aligned}
& \int \delta(\mathrm{N} 1 \geq \mathrm{p} 2 \geq 0) \delta(\mathrm{N} 1 \geq \mathrm{p} 3 \geq 0) \mathrm{K}(\mathrm{p} 2, \mathrm{p} 3)= \\
& {[\delta(2 \mathrm{~N} 1 \geq \mathrm{pa} \geq 0) \delta(\min [\mathrm{N} 1, \mathrm{pa}] \geq \mathrm{p} 2>\max [0, \mathrm{pa}-\mathrm{N} 1]) \mathrm{K}(\mathrm{p} 2, \mathrm{pa}-\mathrm{p} 2)}
\end{aligned}
$$


Defining $p s \equiv p a+p 1$ and replacing 'pa' results in:

$\sum \delta(2 \mathrm{~N} 1 \geq \mathrm{pa} \geq 0) \delta(\mathrm{N} 1 \geq \mathrm{pl} \geq 0) K(\mathrm{pa}, \mathrm{p} 1)=$

$[\delta(3 N 1 \geq p s \geq 0) \delta(\min [N 1, p s] \geq p 1 \geq \max [0, p s-2 N 1]) K(p s-p 1, p 1)$

Noting that $p s \equiv p 1+p 2+p 3$, thus:

$$
\delta(p 1+p 2+p 3 \geq p-1)=\delta(p s \geq p-1)
$$

so that $\{$ Term 3$\}$. can be transformed into:

$$
\begin{aligned}
& \{\text { Term } 3\}=+(B / 4) 2^{\mathrm{P}}\left[\delta(3 \mathrm{~N} 1 \geq \mathrm{ps} \geq \mathrm{p}-1) \mathrm{RC}(\mathrm{p}, \mathrm{ps}) / \Gamma^{\mathrm{ps}-\mathrm{p}+2}\right. \\
& \mathrm{RC}(\mathrm{p}, \mathrm{ps})=[\delta(\min [\mathrm{N} 1, \mathrm{ps}] \geq \mathrm{p} 1 \geq \max [0, \mathrm{ps}-2 \mathrm{~N} 1]) \times \\
& \delta(\min [\mathrm{N} 1, \mathrm{ps}-\mathrm{p} 1] \geq \mathrm{p} 2 \geq \max [0, \mathrm{ps}-\mathrm{p} 1-\mathrm{N} 1]) \times \\
& \theta(\mathrm{p} 1, \mathrm{p} 2, \mathrm{ps}-\mathrm{p} 1-\mathrm{p} 2,1,1,1 ; \mathrm{p}, 3) \mathrm{B}_{\mathrm{p} 1 \mathrm{~B}_{\mathrm{p} 2}{ }^{\mathrm{B}}(\mathrm{ps}-\mathrm{p} 1-\mathrm{p} 2)}
\end{aligned}
$$

Notice that both. \{Term 1\} and \{Term 3$\}$ have the common function:

$$
\delta(3 N 1 \geq p s \geq 0) / \Gamma^{p s-p+2}
$$

whereas \{Term 2\} has the function:

$$
\delta(\mathrm{N} 1+\mathrm{NA}-1 \geq \mathrm{ps} \geq 0) / \Gamma^{\mathrm{ps}-\mathrm{p}+5 / 2}
$$

Thus, one can write:

$$
[\dot{e}(p, 3)-e(p-1,3)]=\hat{f}^{-1}(p, n=3 ; A)-\hat{f}^{-1}(p ; n=3 ; B)
$$

where the ' $A$ ' and ' $B$ ' designations are for the integer and half-integer r-series types:

$$
\begin{aligned}
& \hat{f}^{-1}(p, n=3 ; A)=2^{p} \sum \delta(3 N 1 \geq p s \geq 0) \times \\
& {\left[\left(3 \alpha^{2} / 4\right) R A(p, p s)+\delta(p s \geq p-1)(B / 4) R C(p, p s)\right] / \Gamma^{p s-p+2}} \\
& \hat{f}^{-1}(p, n=3 ; B)=2^{p}\left[3 \alpha^{2} / 4\right][\delta(N 1+N A-1 \geq p s \geq 0) \times \\
& R B(p, p s) / \Gamma^{p s-p+5 / 2}
\end{aligned}
$$


Thus, the solution $e(p, 3)$ can be written as:

$$
e(p, 3)=\sum_{q=0}^{p-1} f^{q}(p-q, n=3 ; A)-\sum_{q=0}^{p-1} \tilde{f}^{q}(p-q, n=3 ; B)
$$

The function $\tilde{\mathrm{f}}^{q}(\mathrm{p}-\mathrm{q}, \mathrm{n}=3 ; A)$ requires $\Phi\left(\nu, 0, q ; \Gamma, \Gamma_{0}\right)$ for $v=(p-q-p s-2)$, and $\tilde{f}^{q}(p-q, n=3 ; \dot{B})$ requires $\phi\left(\nu, 0, q ; r, r_{0}\right)$ for $v=(p-q-p s-5 / 2)$. The half-integer case was explicitly presented in (Sec. II, Chapter 6). The full integer case can be written as:

$$
\begin{aligned}
& \Phi\left(p-q-p s-2,0, q ; \Gamma, \Gamma_{0}\right)=\delta(p-1 \geq p s \geq p-q-1)\left[\ln \Gamma-\ln \Gamma_{0}\right] \times \\
& (-1)^{p s+q-p+1} \Gamma^{p-p s-1 /} /(p s+q-p+1) !(p-p s-1) !+ \\
& (1 / q !) \sum_{m=0}^{q}\left({ }^{q}\right) \cdot(-1)^{q-m}(1-\delta(p-m=p s+1)) \\
& \Gamma_{\left(\Gamma^{p-p s-m-1} \sum_{0}^{p-p s-m-1}\right) /(p-p s-m-1)}^{m}
\end{aligned}
$$

which then gives (using $p-q-1 \geq 0$ ):

$$
\begin{aligned}
& \tilde{f}^{q}(p-q, n=3 ; A)=2^{p-q}[\delta(\min [3 N 1, p-1] \geq p s \geq p-q-1) \times \\
& (-1)^{p s+q-p+1} \cdot\left[\left(3 \alpha^{2} / 4\right) \operatorname{RA}(p-q, p s)+(B / 4) R C(p-q, p s)\right] \times \\
& \Gamma^{p-p s-1} \ln \left(\Gamma / \Gamma_{o}\right) /((p s+q-p+1) !(p-p s-1) !)+ \\
& \left(2^{p-q} / q !\right)\left[\delta(3 N 1 \geq p s>0) \delta(q \geq m>0)\left({ }_{m}^{q}\right)(1-\delta(p s+m=p-1)) \times\right. \\
& (-1)^{q-m}\left[\left(3 \alpha^{2} / 4\right) R A(p-q, p s)+(\beta / 4) \delta(p s>p-q-1) R C(p-q, p s)\right] \times \\
& \Gamma^{m}\left[\Gamma^{p-p s-m-1}-\Gamma_{0}^{p-p s-m-1}\right] /(p-p s-m-1)
\end{aligned}
$$

One can further find, for the $v=($ half-integer) case, easily:

$$
\begin{aligned}
& \tilde{f}^{q}(p-q, n=3 ; B)= \\
& \left(3 \alpha^{2} / 4\right) 2^{\mathrm{p}-\mathrm{q}}[\delta(\mathrm{N} 1+\mathrm{NA}-1 \geq \mathrm{ps} \geq 0) \mathrm{R} 3 \mathrm{~B}(\mathrm{p}-\mathrm{q}, \mathrm{ps}) \times \\
& (1 / q !) \sum \delta(q \geq m \geq 0)\left({ }_{m}^{q}\right)(-1)^{q-m} \Gamma^{m}\left[\Gamma^{p-m-p s-3 / 2}-\Gamma_{0}^{p-m-p s-3 / 2}\right] /(p-m-p s-3 / 2)
\end{aligned}
$$


which now gives a complete representation of the functions $e(p, 3)$, in terms of $\cdots\left(\Gamma, \Gamma_{0}, \alpha, \beta\right)$.

One can next rearrange the terms of $e(p, 3)$ to get a sequence in descending powers of $\Gamma$. Using an alternate additive combination on some sums, one has two equivalent expressions:

$$
\begin{aligned}
& \sum \delta(\mathrm{N} \geq \mathrm{ps}>0) \delta(\mathrm{p}-1 \geq \mathrm{m} \geq 0) \mathrm{K}(\mathrm{ps}, \mathrm{m})= \\
& {[\delta(\mathrm{N} \geq \mathrm{ps} \geq 0) \delta(\mathrm{ps}+\mathrm{p}-1 \geq \mathrm{t} \geq \mathrm{ps}) \mathrm{K}(\mathrm{ps}, \mathrm{t}-\mathrm{ps})=} \\
& \sum \delta(\mathrm{p}-1 \geq \mathrm{m} \geq 0) \delta(\mathrm{m}+\mathrm{N} \geq \mathrm{t} \geq \mathrm{m}) \mathrm{k}(\mathrm{t}-\mathrm{m}, \mathrm{m}),
\end{aligned}
$$

which give terms in either $(m, t)$-indices with $\Gamma^{m}$ powers or terms in (ps,t)-indices with $\Gamma^{\mathrm{p}-\mathrm{ps}-1}$ powers (and one term with $\Gamma^{\mathrm{p}-\mathrm{ps}-3 / 2}$ powers). In all the $m$-sums, the range is $m=(0, p-1)$ so summing in reverse order gives powers of $\Gamma^{\mathrm{p}-\mathrm{m}-1}$ instead of powers of $\cdot \Gamma^{\mathrm{m}}:$ Then, changing the remaining label: ('ps' or ' $m$ ') to ' $r$ ', one can get:

$$
\begin{aligned}
& e(p, 3)=\left(3 \alpha^{2} / 4\right) \cdot\left[\delta(\max [3 N 1, p-1] \geq r \geq 0) \Gamma^{p-r-1} s 3 A(p, r)+\right. \\
& \sum \delta(\min [3 \mathrm{~N} 1, \mathrm{p}-1] \geq \mathrm{r} \geq 0) \Gamma^{\mathrm{p}-\mathrm{r}-1} \cdot \operatorname{SAl}(\mathrm{p}, \mathrm{r}) \ln \left(\Gamma / \Gamma_{0}\right)- \\
& {\left[\delta(N 1+N A-1 \geq r \geq 0) \Gamma^{\mathrm{P}-\mathrm{r}-3 / 2} \operatorname{SA} 2(\mathrm{p}, \mathrm{r})\right\}+} \\
& (B / 4)\left\{\left[\delta(\max [3 N 1, p-1] \geq r \geq 0) \Gamma^{p-r-1} s 3 B(p, r)+\right.\right. \\
& \left\{\delta(\min [3 N 1, p-1] \geq r \geq 0) \Gamma^{p-r-1} \operatorname{sB} 1(p, r) \ln \left(\Gamma / \Gamma_{0}\right)\right\}
\end{aligned}
$$

where the explicit form of the arrays 'S3A', 'SA1', 'SA2', 'S3B', 'SBI' are given in the next (summary) section. From $e(p, 3)$, one can easily construct the $g(p, 3)$ :

$$
g(p, 3)=(1 / 2)^{p} \Gamma^{-(p+1 / 2)} \text { e }(p, 3)
$$


which then are:

$$
\begin{aligned}
& g(p, 3)=(3 \alpha 2 / 4) 2^{-p}\left\{\left[\delta(\max [3 N 1, p-1] \geq r>0) S 3 A(p, r) \Gamma^{-r-3 / 2}+\right.\right. \\
& \left\{\delta(\min [3 N 1, p-1] \geq r \geq 0) \operatorname{SA} 1(p, r) \Gamma^{-r-3 / 2} \ln \left(\Gamma / \Gamma_{0}\right)-\right. \\
& \left\{\delta(N 1+N A-1 \geq r \geq 0) S A 2(p, r) \Gamma^{-r-2}\right\}+ \\
& (B / 4) 2^{-p}\left\{\left[\delta(\max [3 N 1, p-1] \geq r \geq 0) \sin (p, r) \Gamma^{-r-3 / 2}+\right.\right. \\
& {\left[\delta(\min [3 N 1, p-1] \geq r \geq 0) \operatorname{SB} 1(p, r) \Gamma^{-r-3 / 2} \ln \left(\Gamma / \Gamma_{0}\right)\right\}}
\end{aligned}
$$

Notice, as with the $g(p, 2)$ function, this is entirely expressed as inverse powers of $\Gamma$ (with a $\ln \left(\Gamma / \Gamma_{0}\right)$ term). Since ' $\Gamma$ ' is linearly related to time via. $\Gamma=a\left(t+t_{0}\right)$, the slowest decaying portion of $g(p, 2)$ and of $" g(p, 3)$ is a term:

$$
g(p, n \neq 1) \sim \Gamma^{-3 / 2}
$$

whereas the linear equation (embodied by $g(p, 1)$ ) had terms:

$$
g(p, 1) \sim \Gamma^{-(p+1 / 2)}
$$

Thus, for large $\Gamma$ (long time), the dominant term must be $g(0,1)$, which is the pure gaussian.

Since all $g(p, n \neq 1)$ begin $\left(\right.$ as $\left.\Gamma \rightarrow \Gamma_{0}\right)$ at zero, and they all decay faster than the gaussian (for large $\Gamma$ ), all series converge!

Finally; because every non-linear term $(g(p, n \neq 1)$ function $)$ decays as fast as $\sim \dot{\Gamma}^{-3 / 2}$ (with at most $\left[\ln \left(\Gamma / \Gamma_{0}\right)\right]^{\mathrm{m}}$ corrections), when the solution is re-coupled together and expressed as the single series:

$$
C(z, \Gamma)=\sum G_{m}(\Gamma) H_{2 m}(z) \exp -z^{2}
$$

The $\left(G_{m}(\Gamma), m \neq 0\right)$ terms which would have decayed via $\Gamma^{-(m+1 / 2)}$ for $D=$ constant, now ali decay via $\sim \Gamma^{-3 / 2}$. Thus, the non-linearities prevent 
decay of the non-gaussian terms, thereby allowing persistence of the "curvature" on a [lnC vs. $\left.x^{2}\right]$-plot for a long time.

\section{Summary of Calculations}

A given normed state:

$$
C_{R}\left(x, t_{R}\right)=\sum_{m=0}^{N 1} B_{m} H_{2 m}\left(x / x_{R}\right) \exp -\left(x / x_{R}\right)^{2}
$$

which propagates forward via the non-linear equation:

$$
\frac{\partial C}{\partial t}=D_{0} \frac{\partial}{\partial x}-\left(1+\alpha C+\beta C^{2}\right) \frac{\partial C}{\partial x}
$$

gives an equation at a time $t_{F}$ :

$$
\begin{aligned}
& \hat{C}_{\dot{R}}\left(x, t_{F}\right)=\sum_{m=0}^{m(\max )} G_{m}(\Gamma) H_{2 m}(z) \exp -z^{2} \\
& \Gamma=1+4 D_{0}\left(t_{F}-t_{R}\right) / x_{R}^{2} \\
& z^{2}=x^{2} / \Gamma x_{R}^{2}
\end{aligned}
$$

In principle, the sum on ' $m$ ' ranges $m=(0, \infty)$ even for a finite $N 1$ in the $C_{R}\left(x, t_{R}\right)$ state. However, as noted before (Sec. VIII, Chapter 4), a consistent leve1 of approximation sets $\operatorname{m(max)}$ approximately equal to 'N1'. The $G_{m}(\Gamma)$ are given by:

$$
G_{m}(\Gamma)=\sum_{n=1}^{n(\max )} n^{-m-1 / 2} \sum_{p=0}^{m} g(p, n)[(1-n) / 4]^{m-p} /(m-p) !
$$

We have solved for $g(p, n)$ for $n(\max )=3(n=1,2,3)$ to give $c(2, \Gamma)$. up to order $\left(\alpha^{2}, \beta\right)$ The equation for $g(p, 1)$ is simply:

$$
g(p, 1)=B_{p} / \Gamma^{p+1 / 2}
$$


The equation for $g(p, 2)$ is:

$$
g(p, 2)=\alpha 4^{-p}\left\{\sum_{r=0}^{2 N 1} \Gamma^{-r-1} s 2 A(p, r)-\sum_{r=0}^{p-1} \Gamma^{-r-3 / 2}\left(\Gamma_{0}^{r-p+1}\right) S 2 B(p, p-r-1)\right\}
$$

where:

$$
\begin{aligned}
& R 2(p, p a)=\sum \delta(m i n[p a, N 1] \geq p 1 \geq \max [0, p a-N 1]) \times \\
& \theta(p 1, p a-p 1,1,1 ; p, 2){ }_{p 1}^{B}(p a-p 1) \\
& S 2 A(p, p a)=\sum \delta(p-1 \geq m>0)(p-p a-m-1 / 2)^{-1} / m ! \times \\
& \sum \delta(p-1 \geq q>\max [m, p-p a-1]) 4^{p-q-1} R 2(p-q, p a)(-1)^{q-m} /(q-m) ! \\
& S 2 B(p, m)=\sum \delta(2 N 1 \geq p a \geq 0) \Gamma_{0}^{p-p a-1 / 2}(p-p a-m-1 / 2)^{-1} / m ! \times \\
& \sum \delta(p-1 \geq q>\max [m, p-p a-1]) 4^{p-q-1} R 2(p-q, p a)(-1)^{q-m} /(q-m) !
\end{aligned}
$$

And the equation for $g(p, 3)$ is:

$$
\begin{aligned}
& g(p, 3)=(3 \alpha 2 / 4) 2^{-p}\left\{[\delta(\max [3 N 1, p-1] \geq r \geq 0]) S 3 A(p, r) \Gamma^{-r-3 / 2}+\right. \\
& {\left[\delta(\min [3 N 1 ; p-1] \geq r \geq 0) \operatorname{SA} 1(p, r) \Gamma^{-r-3 / 2} \ln \left(\Gamma / \Gamma_{0}\right)-\right.} \\
& {\left[\delta(N 1+N A-1 \geq r \geq 0) . S A 2(p, r) \Gamma^{-r-2}\right\}+} \\
& (B / 4) 2^{-p}\left\{\left[\delta(\max [3 N 1, p-1] \geq r \geq 0) S 3 B(p, r) \Gamma^{-r-3 / 2}+\right.\right. \\
& {\left[\delta(\min [3 N 1, p-1] \geq r \geq 0) S B 1(p, r) \Gamma^{-r-3 / 2} \ln \left(\Gamma / \Gamma_{0}\right)\right\}}
\end{aligned}
$$

where:

$$
\begin{aligned}
& \mathrm{RA}(\mathrm{p}, \mathrm{ps})=\sum \delta(\min [2 \mathrm{~N} 1, \mathrm{ps}] \geq \mathrm{pa} \geq \max [0, \mathrm{ps}-\mathrm{N} 1]) \mathrm{B}(\mathrm{ps}-\mathrm{pa}) \\
& \int \delta(\mathrm{NA} \geq \mathrm{p} 2 \geq \max [1, \mathrm{p}+\mathrm{pa-ps}-1]) \times \\
& 4^{-\mathrm{p} 2} \dot{\theta}(\mathrm{ps}-\mathrm{pa}, \mathrm{p} 2,1,2 ; \mathrm{p}, 3) \mathrm{s} 2 \mathrm{~A}(\mathrm{p} 2, \mathrm{pa})
\end{aligned}
$$




$$
\begin{aligned}
& \mathrm{RB}(\mathrm{p}, \mathrm{ps})=\sum \delta(\min [\mathrm{N} 1, \mathrm{ps}] \geq \mathrm{p} 1 \geq \max [0, \mathrm{ps}-\mathrm{NA}+1]) \mathrm{B}_{\mathrm{p} 1} \times \\
& {[\delta(\mathrm{NA} \geq \mathrm{p} 2 \geq \max [\mathrm{ps}+1, \mathrm{p}-1]-\mathrm{p} 1) \theta(\mathrm{p} 1, \mathrm{p} 2,1,2 ; \mathrm{p}, 3) \times} \\
& 4^{-\mathrm{p} 2} \Gamma^{-(\mathrm{p} 1+\mathrm{p} 2-\mathrm{ps}-1)} \mathrm{s} 2 \mathrm{~B}(\mathrm{p} 2, \mathrm{p} 1+\mathrm{p} 2-\mathrm{ps}-1) \\
& \mathrm{RC}(\mathrm{p}, \mathrm{ps})=\sum \delta(\min [\mathrm{N} 1, \mathrm{ps}] \geq \mathrm{p} 1 \geq \max [0, \mathrm{ps}-2 \mathrm{~N} 1]) \times \\
& \delta(\min [\mathrm{N} 1, \mathrm{ps}-\mathrm{p} 1] \geq \mathrm{p} 2 \geq \max [0, \mathrm{ps}-\mathrm{p} 1-\mathrm{N} 1]) \times \\
& \Theta(\mathrm{p} 1, \mathrm{p} 2, \mathrm{ps}-\mathrm{p} 1-\mathrm{p} 2,1,1,1 ; \mathrm{p}, 3) \mathrm{B}_{\mathrm{p} 1 .} \mathrm{B}_{\mathrm{p} 2} \mathrm{~B}_{(\mathrm{ps}-\mathrm{p} 1-\mathrm{p} 2)}
\end{aligned}
$$

and where:

$$
\begin{aligned}
& \operatorname{S} 3 A(p, r)= \\
& {\left[\delta ( 3 N 1 \geq r ) \left[\delta(r+p-1 \geq t \geq r)(1-\delta(t=p-1))(p-t-1)^{-1} \times\right.\right.} \\
& {\left[\delta(p-1 \geq q \geq t-r)\left(2^{p-q} /(t-r) !(q+r-t) !\right)(-1)^{q+r-t} R A(p-q, r)\right]+} \\
& {\left[\delta ( p - 1 \geq r ) \left[\delta(N 1+N A+p-r-2 \geq t>p-r-1) \Gamma_{0}^{p-t-3 / 2}(p-t-3 / 2)^{-1} \times\right.\right.} \\
& {\left[\delta(r \geq s \geq 0)\left(2^{r-s-1} / s !(p-r-1) !\right)(-1)^{s} R B(r-s+1, r+t-p+1)\right]-} \\
& {\left[\delta ( \mathrm { p } - 1 \geq \mathrm { r } ) \left[\delta(3 \mathrm{~N} 1+\mathrm{p}-\mathrm{r}-1 \geq \mathrm{t} \geq \mathrm{p}-\mathrm{r}-1)(1-\delta(\mathrm{t}=\mathrm{p}-1)) \Gamma_{0}^{\mathrm{p}-\mathrm{t}-1}(\mathrm{p}-\mathrm{t}-1)^{-1} \times\right.\right.} \\
& {\left[\delta(r \geq s \geq 0)\left(2^{r-s-1} / s !(p-r-1) !\right)(-1)^{s} R A(r-s+1, r+t-p+1)\right]} \\
& \operatorname{SAl}(\mathrm{p}, \mathrm{r})=\left((-1)^{\dot{p}-\mathrm{r}-1} /(\mathrm{p}-\mathrm{r}-1) !\right) \times \\
& \sum \delta(p-1 \geq q \geq p-r-1) \cdot(-1)^{q} 2^{p-q} R A(p-q, r) /(q-p+r+1) ! \\
& \operatorname{SA} 2(p, r)= \\
& \sum \delta(r+p-1 \geq t \geq r)(p-t-3 / 2)^{-1}[\delta(p-1 \geq q>t-r) x \\
& \left(2^{p-q} /(t-r) !(q+r-t) !\right)(-1)^{q+r-t} R B(p-q, r)
\end{aligned}
$$




$$
\begin{aligned}
& \operatorname{S3B}(p, r)= \\
& {\left[\delta(3 N 1 \geq r) \sum \delta(r+p-1 \geq t \geq r)(1-\delta(t=p-1))(p-t-1)^{-1} \times\right.} \\
& \quad\left[\delta(p-1 \geq q \geq \max [t, p-1]-r)(-1)^{q+r-t} 2^{p-q} \times\right. \\
& R C(p-q, r) /(t-r) !(q+r-t) !]- \\
& {\left[\delta ( p - 1 \geq r ) \left[\delta(3 N 1+p-r-1 \geq t \geq p-r-1)(1-\delta(t=p-1)) \Gamma_{0}^{p-t-1}(p-t-1)^{-1} \times\right.\right.} \\
& \quad\left[\delta(r \geq s>\max [0, p-t-1])\left(2^{r-s-1} / s !(p-r-1) !\right)(-1)^{s} R C(r-s+1, r+t-p+1)\right] \\
& S B 1(p, r)=\left((-1)^{p-r-1} /(p-r-1) !\right) \times \\
& \quad\left[\delta(p-1 \geq q \geq p-r-1)(-1)^{q}{ }_{2}^{p-q} R C(p-q, r) /(q-p+r+1) !\right.
\end{aligned}
$$

As every array is composed entirely of parameters defined by the initial state, $C_{R}\left(x, t_{R}\right)$, this analysis naturally lends itself to computations via computer assistance.

\section{v. Non-1inear and Multiple-time Correlations}

Having solved the non-linear equation $D(C)=\left(1+\alpha C+B C^{2} \ldots\right)$ via construction of the $g(p, n)$-functions; one can find the manner by which a given $C\left(x, t_{i}\right)$ state will propagate forward in time.

The definitions of the dynamical variables $(\Gamma, z)$ as in (Sec. IV, Chapter 4):

$$
\begin{aligned}
& \Gamma=1+4 D\left(t_{F}-t_{R}\right) / x_{R}^{2} \\
& z^{2}=x^{2} / \Gamma x_{R}^{2}
\end{aligned}
$$

require an elaboration when extended to handle the situation of multiple profiles: $\tilde{C}\left(x, t_{i}\right), i=(1,2 \ldots)$. One supposes that one has measured a set of $\tilde{c}\left(x, t_{i}\right)$-states $(i=1,2 \ldots)$, each state having its own characteristic 
expansion parameter $x_{R}(i)$, and 1 ts own normalization $N(i): \int \tilde{C}\left(x, t_{i}\right) d x=$ $N(i)$. The normed profiles $c\left(x, t_{i}\right)=\tilde{C}\left(x, t_{i}\right) / N(i)$ and average normalization $\overline{\mathrm{N}}$ having been created (Sec. II, Chapter 4), we introduce the notation:

$$
\begin{gathered}
\Gamma(k, i)=1+4 D\left(t_{k}-t_{i}\right) /\left(x_{R}(i)\right)^{2} \\
\because \\
\text { Define: } \hat{C}_{i}\left(x, t_{k}\right) \equiv C(x, \Gamma(k) ; i)
\end{gathered}
$$

where $C(x, \Gamma(k) ; i)$ represents the $C(i)^{\text {th }}$ state propagated forward to the time $t_{k}$, and where the $(\Gamma(k, i) ; i)$ notation, being redundant, has been shortened to: $(\Gamma(k) ; i)$. Similarly $(\Gamma(i) ; i)$ is redundant and will be denoted $\left(\Gamma_{0} ; 1\right)$. One can then correlate the $k^{\text {th }}$-state and the $i^{\text {th }}$-state by minimizing:

$$
\begin{aligned}
& \Phi^{2}=\int\left[C\left(x, \Gamma_{0} ; k\right)-C(x, \Gamma(k) ; i)\right]^{2} d x / \int[C(x, \Gamma ; k)]^{2} d x= \\
& 1+\int\left[(C(x, \Gamma(k) ; i))^{2}-2 C(x, \Gamma(k) ; i) C\left(x, \Gamma_{0} ; k\right)\right] d x / \int[C(x, \Gamma ; k)]^{2} d x
\end{aligned}
$$

If one measures $N$-states: $C\left(x, t_{i}\right) ; i=(1,2, \ldots \vdots N)$, one can create a minimizing functional involving up to $\frac{1}{2} \mathrm{~N}(\mathrm{~N}+1)$ pair-wise correlations using each distinct pair $\left[C\left(x, \Gamma_{0} ; k\right), C(x, \Gamma(k) ; i)\right]$. If one performs a correlation involving 'NK' number of terms, it can be represented by:

$$
\Phi^{2}=\frac{1}{(N K)} \sum_{j=1}^{N K}\left(1+\frac{\int\left[\left(C\left(x, \Gamma\left(k_{j}\right) ; k_{j}^{o}\right)\right)^{2}-2 C\left(x, \Gamma\left(k_{j}\right) ; k_{j}^{o}\right) c\left(x, \Gamma_{o} ; k_{j}\right) j d x\right.}{\int\left[C\left(x, \Gamma_{0} ; k_{j}\right)\right]^{2} d x},(6.79)\right.
$$

where ' $k_{j}^{0}$ ' labels the initial state, and ' $k_{j}$ ' labels the final state; and where ' $\mathrm{j}$ ' labels the correlation. The distinct advantage of the choice of dynamical variables $(\Gamma, z)$ becomes clear. The same values of the physical parameters $\left(D_{0}, \alpha, \beta \ldots\right)$ must apply to every state and every propagation, and every $\Gamma(i, k)$ is thus a linear functional of the same parameter $D$. 
Inherently,

$$
\phi^{2}=\phi^{2}(D, \alpha, \beta, \ldots)
$$

where the particular value of D which mintmizes $\Phi^{2}$ is the derived value of $\mathrm{D}_{\mathrm{O}}$

For the particular model $D(C)=D_{0}\left(1+\alpha C+\beta C^{2}\right)$, with ' $C$ ' expanded out to order $-\left(\alpha^{2}, \beta\right)$ (which solves $g(p, n)$ for $n=(1,2,3)$ ), we represent

$$
\begin{aligned}
& c\left(x, \Gamma_{o} ; k_{j}\right)=\phi_{O O}(j)=[\text { final state] } \\
& c\left(x, \Gamma\left(k_{j}\right) ; k_{j}^{o}\right)=[\text { propagated state }]= \\
& \phi_{0}(j)+\alpha \phi_{1}(j)+\alpha^{2} \phi_{2}(j)+\beta \phi_{3}(j)
\end{aligned}
$$

where the ' $\phi^{\prime}$ s are given by the analysis of last chapter. Direct substitution into the equation for $\Phi^{2}$ given the following results (definitions proceed after Model (6) ):

Model (1). For the linear equation: $D(C)=D_{0}$ the minimizing functional: $(\mathrm{Q} 1)^{2}$ is :

$$
(\mathrm{Q} 1)^{2}=[1+\mathrm{Y} 1]
$$

Model (2). For the $D(C)=D_{0}(1+\alpha C)$ equation, $C(z, \Gamma)$ truncated to order $-(\alpha)$ is:

$$
C\left(x, \Gamma\left(k_{j}\right) ; k_{j}^{o}\right)=\phi_{0}(j)+\alpha \phi_{1}(j)
$$

(so that one can quantify the effect of not calculating $c(z, \dot{\Gamma})$ to all orders in $(\alpha)$, i.e., not calculating $g(p, n)$ for all $n)$, and the minimizing functional: $(\mathrm{Q} 2)^{2}$ is

$$
(\mathrm{Q} 2)^{2}=(\mathrm{Q} 1)^{2}+\alpha(\mathrm{Y} 2)+\alpha^{2}(\mathrm{Y} 3) \text {. }
$$


One determines $\alpha$ via:

$$
\frac{\partial}{\partial \alpha}\left(\mathrm{Q}^{2}\right)^{2}=0 ; \quad \alpha=-(\mathrm{Y} 2) / 2(\mathrm{Y} 3)
$$

which gives a modified $\left(Q_{2}\right)^{2}$ :

$$
\left(Q^{2}\right)^{2}=(Q 1)^{2}-(Y 2)^{2} / 4(Y 3)
$$

Model (3). Again, for the $D(C)=D_{0}(1+\alpha C)$ equation, with $C(z, \Gamma)$ calculated to order $-\left(\alpha^{2}\right)$ :

$$
C\left(x ; \Gamma\left(k_{j}\right) ; k_{j}^{o}\right)=\phi_{0}(j)+\alpha \phi_{1}(j)+\alpha^{2} \phi_{2}(j)
$$

(so that one may compare this to Model (2)), the minimizing functional $(\mathrm{Q} 3)^{2}$ is:

$$
(\mathrm{Q} 3)^{2}=(\mathrm{Q})^{2}+\alpha(\mathrm{Y} 2)+\dot{\alpha}^{2}(\mathrm{Y} 3+\mathrm{Y} 4)+\alpha^{3}(\mathrm{Y} 5)+\alpha^{4}(\mathrm{Y} 6)
$$

One determines $\alpha$ as the root of a cubic, via:

$$
\frac{\partial}{\partial \alpha}(\mathrm{Q} 3)^{2}=0 ; \quad(Y 2)+2 \alpha(Y 3+Y 4)+3 \alpha^{2}(Y 5)+4 \alpha^{3}(Y 6)=0
$$

which gives a modified $(\mathrm{Q} 3)^{2}$ :

$$
(\mathrm{Q} 3)^{2}=(\mathrm{Q} 1)^{2}+(3 \alpha / 4)(Y 2)+\left(\alpha^{2} / 2\right)(Y 3+Y 4)+\left(\alpha^{3} / 4\right)(Y 5) .
$$

Model (4). For the $D(C)=D_{0}\left(1+B C^{2}\right)$ equation, the first non-1inear correction to $C(z ; \Gamma)$ is:

$$
C\left(x, \Gamma\left(k_{j}\right) ; k_{j}^{o}\right)=\phi_{0}(j)+\beta \phi_{3}(j)
$$

(so that one may compare this model which is not expected to be motivated by the basic physics, with the others). It can provide a measure of how much better a data-fit can be obtained by using any non-linear correction, and the minimizing functional, $\left(Q_{4}\right)^{2}$, is:

$$
(Q 4)^{2}=(Q 1)^{2}+\beta(Y 7)+\beta^{2}(Y 8) .
$$


One determines $\beta$ via:

$$
\frac{\partial}{\partial \beta} \cdot(Q 4)^{2}=0 ; \quad B=-(Y 7) / 2(Y 8)
$$

which gives a modified $(Q 4)^{2}$ :

$$
(\mathrm{Q} 4)^{2}=(\mathrm{Q} 1)^{2}-(\mathrm{Y} 7)^{2} / 4(\mathrm{Y} 8) \text {. }
$$

Model (5). For the $D(C)=D_{0}\left(1+\alpha C+\beta C^{2}\right)$ equation, with $C(z, \Gamma)$ calculated to order $-\left(\alpha^{2}, \beta\right)$ :

$$
C\left(x, \Gamma\left(k_{j}\right) ; k_{j}^{o}\right)=\phi_{o}(j)+\alpha \phi_{1}(j)+\alpha^{2} \phi_{2}(j)+\beta \phi_{3}(j),
$$

the minimizing functional: $(Q 5)^{2}$ is:

$$
\begin{aligned}
& (\mathrm{Q} 5)^{2}=(\mathrm{Q} 1)^{2}+\alpha(\mathrm{Y} 2)+\alpha^{2}(Y 3+Y 4)+\alpha^{3}(Y 5)+\alpha^{4}(Y 6)+ \\
& B(Y 7)+\beta^{2}(Y 8)+\alpha \beta(Y 9)+\alpha^{2} \beta(Y 10) .
\end{aligned}
$$

One determines $\beta$ as a function of $\alpha$ via:

$$
\frac{\partial}{\partial \beta}(Q 5)^{2}=0 ; \beta=-\left[(Y 7)+\alpha(Y 9)+\alpha^{2}(Y 10)\right] / 2(Y 8)
$$

which gives a modified $(Q 5)^{2}$ :

$$
\begin{aligned}
& (\mathrm{Q} 5)^{2}=\left[(\mathrm{Q} 1)^{2}-(\mathrm{Y} 7)^{2} / 4(\mathrm{Y} 8)\right]+\alpha[(\mathrm{Y} 2)-(\mathrm{Y} 7)(\mathrm{Y} 9) / 2(\mathrm{Y} 8)]+ \\
& \alpha^{2}\left[(\mathrm{Y} 3)+(\mathrm{Y} 4)-\left((\mathrm{Y} 9)^{2} / 4(\mathrm{Y} 8)\right)-((\mathrm{Y})(\mathrm{Y} 10) / 2(\mathrm{Y} 8))\right]+ \\
& \alpha^{3}[(\mathrm{Y} 5)-(\mathrm{Y} 9)(\mathrm{Y} 10) / 2(\mathrm{Y} 8)]+\alpha^{4}[(\mathrm{Y} 6)-(\mathrm{Y} 10) / 4(\mathrm{Y} 8)]
\end{aligned}
$$

where $\alpha$ is then isolated by a process similar to (Q3) ${ }^{2}$.

Model (6). For the $D(C)=D_{0}\left(1+\alpha C+p \alpha^{2} C^{2}\right)$ equation, where ' $p$ ' is fixed a-priori (for example: $D(C)=D_{0}\left(\frac{1}{2} \alpha C+\left[1+\left(\frac{1}{2} \alpha C\right)^{2}\right]^{1 / 2}\right) \simeq$ $\mathrm{D}_{\mathrm{o}}\left(1+(\alpha \mathrm{C} / 2)+(\alpha \mathrm{C})^{2} / 8+\ldots\right)$ would give $\left.\mathrm{p}=\frac{1}{2}\right)$, with $\mathrm{C}(\mathrm{z}, \Gamma)$ calculated 
to order $-\left(\alpha^{2}\right)$ :

$$
C\left(x, \Gamma\left(k_{j}\right) ; k_{j}^{o}\right)=\phi_{0}(j)+\alpha \phi_{1}(j)+\alpha^{2}\left[\phi_{2}(j)+p \phi_{3}(j)\right],
$$

the minimizing functional: $(Q 6)^{2}$ is:

$$
\begin{aligned}
& (\mathrm{Q} 6)^{2}=(\mathrm{Q} 1)^{2}+\alpha(\mathrm{Y} 2)+\alpha^{2}[(\mathrm{Y} 3)+(\mathrm{Y} 4)+\mathrm{p}(\mathrm{Y} 7)] \\
& \alpha^{3}[(\mathrm{Y} 5)+\mathrm{p}(\mathrm{Y} 9)]+\alpha^{4}\left[(\mathrm{Y} 6)+\mathrm{p}(\mathrm{Y} 10)+\mathrm{p}^{2}(\mathrm{Y} 8)\right]
\end{aligned}
$$

and where $\alpha$ is isolated by a process similar to $(Q 3)^{2}$.

The terms which appear in the minimizing functional are of the form:

$$
\begin{aligned}
& \int\left[C\left(x, \Gamma_{0} ; k_{j}\right)\right]^{2} d x=\int\left[\phi_{o o}(j)\right]^{2} d x \equiv Z N(j) \\
& \int\left[C\left(x, \Gamma_{0} ; k_{j}\right) C\left(x, \Gamma\left(k_{j}\right) ; k_{j}^{\prime}\right)\right] d x= \\
& \int\left[\phi_{o o}(j)\left(\phi_{o}(j)+\alpha \phi_{1}(j)+\alpha^{2} \phi_{2}(j)+\beta \phi_{3}(j)\right)\right] d x \\
& \int\left[C\left(x, \Gamma\left(k_{j}\right) ; k_{j}\right]^{2} d x=\right. \\
& \int\left[\phi_{o}(j)+\alpha \phi_{1}(j)+\alpha^{2} \phi_{2}(j)+\beta \phi_{3}(j)\right]^{2} d x .
\end{aligned}
$$

The definitions of the functions $[(\mathrm{Y} 1),(\mathrm{Y} 2), \ldots(\mathrm{Y} 10)]$ used in the functionals $\left[(Q 1)^{2},(Q 2)^{2}, \ldots(Q 6)^{2}\right]$ are the following:

$$
\begin{aligned}
& (Y 1)=(N K)^{-1}\left[[Z N(j)]^{-1} \int\left[\phi_{0}(j)-2 \phi_{O O}(j)\right]\left[\phi_{0}(j)\right] d x\right. \\
& (Y 2)=(N K)^{-1}\left[[Z N(j)]^{-1} \int\left[2 \phi_{0}(j)-2 \phi_{O O}(j)\right]\left[\phi_{1}(j)\right] d x\right. \\
& (Y 3)=(N K)^{-1}\left[[Z N(j)]^{-1} \int\left[\phi_{1}(j)\right]^{2} d x\right. \\
& (Y 4)=(N K)^{-1}\left[[Z N(j)]^{-1} \int\left[2 \phi_{0}(j)-2 \phi_{O O}(j)\right]\left[\phi_{2}(j)\right] d x\right. \\
& (Y 5)=(N K)^{-1}\left[[Z N(j)]^{-1} \int\left[2 \phi_{1}(j) \phi_{2}(j)\right] d x\right.
\end{aligned}
$$




$$
\begin{aligned}
& (Y 6)=(N K)^{-1}\left[[\mathrm{ZN}(j)]^{-1} \int\left[\phi_{2}(j)\right]^{2} d x\right. \\
& (Y 7)=(N K)^{-1} \sum[\mathrm{ZN}(j)]^{-1} \int\left[2 \phi_{0}(j)-2 \phi_{O O}(j)\right]\left[\phi_{3}(j)\right] d x \\
& (Y 8)=(N K)^{-1} \sum[\mathrm{ZN}(j)]^{-1} \int\left[\phi_{3}(j)\right]^{2} d x \\
& (Y 9)=(N K)^{-1} \sum[\mathrm{ZN}(j)]^{-1} \int\left[2 \phi_{1}(j) \phi_{3}(j)\right] d x \\
& (Y 10)=(N K)^{-1}\left[[Z N(j)]^{-1} \int\left[2 \phi_{2}(j) \phi_{3}(j)\right] d x\right.
\end{aligned}
$$

where the implicit limits on each sum are $j=(1, N K)$.

This now gives $\Phi^{2}$, entirely as a function of the parameter $D$, and a search in the range of $D$ for the value $D_{0}$ which minimizes $\Phi^{2}$ finally determines $D_{0}$, thus determining the entire function:

$$
D(C)=D_{0}\left(1+\alpha C+B C^{2}\right)
$$

And, by using the average normalization $\bar{N}$, one can find $\left(\alpha_{0}, \beta_{0}\right)$ which are the non-linear parameters that are independent of total impurity amount: $\quad\left(\alpha_{0}=\alpha / \overline{\mathrm{N}}, \beta_{0}=\dot{\beta} / \overline{\mathrm{N}}\right)$.

Finally, because all the integrals $[(Y 1),(Y 2),(Y 3), \ldots(Y 10)]$ originate from products of concentrations, they can all be written in terms of:

$$
I_{m, n}(a, b)=\int H_{2 m}(a \xi) H_{2 n}(b \xi) \delta\left(a^{2}+b^{2}=1\right) \exp -\left(\xi^{2}\right) d \xi
$$

exactly as encountered in the linear equation (see Eq. (2.35) ) and which has been calculated ${ }^{20}$ (see Eq. (2.36) ). 


\section{CHAPTER 7}

\section{EXPERIMENTAL METHOD}

\section{Experimental Procedures}

\section{A. Crystal Cleaving}

Crucial to any diffusion experiment is careful handling of crystals and of the tracer deposition process before the diffusion anneal.

The large crystals used in this experiment were either from a 1 "cleaved cube single crystal, or from string-saw cut rectangular parallelopipeds, both high-purity $\mathrm{NaCl}$, supplied by Harshaw Chemical Co. The samples arrived encased in polyethylene and were not opened until use. Cleaving was done entirely inside a large polyethylene glove-bag attached directly to a vacuum evaporator where the tracer deposition was done. The glove bag was triple-flushed with nitrogen dried with an ethanol-solid $\mathrm{CO}_{2}$ cold-trap before use.

A crystal cleaver was constructed, primarily consisting of various shaped crystal holders, and "a razor-blade holder-aligner; and it could "size" a small crystal to within a fraction of a millimeter. Crystals approximately $7.5 \mathrm{~mm} \times 7.5 \mathrm{~mm} \times(2-3 \mathrm{~mm})$ were prepared with this assembly, using a stainless-steel rod as a "hammer". Razor blades were degreased and washed in acetone prior to use.

Since polyethylene is slightly cloudy, a small lucite "window" was put on the glove bag so that the cleaved surfaces could be examined using light reflection. They showed at most a few striations, indicating 
jogs in the crystal planes. The first "slice" of each data-set was powder and its weight.indicates an upper bound to the average "roughness" of the surface. This was characteristically an 8 micron slice-weight, but often it was less. The cleaved surfaces were not microtome-polished, since earlier work ${ }^{51}$. found surface iron-impurities are probably introduced by this process. Not microtome-polishing also eliminates the need for a special pre-tracer-deposition anneal to heal out microtome-strains.

$$
* * *
$$

\section{B. Tracer Amounts}

Crucial to a concentration-dependent diffusion experiment is an accurate knowledge of the total amount of impurity initially deposited on the crystal surface before the high-temperature diffusion anneal. This is because the total impurity amount determines the magnitude of the nonlinearity parameters, irréspective of diffusion anneal time (see Sec. II, Chapter 4).

$\mathrm{Ca}^{45}$ tracer, a $\beta$-emitter (ha1f life $=165.0$ days), in the form of $\mathrm{CaCl}_{2}$ in . $5 \mathrm{~N}-\mathrm{HCl}$ was obtained from New England Nuclear Company: Guaranteed $99+\%$ radiometric purity, the tracer could have trace non-radioactive impurities. Whereas a sma11 amount of non-radioactive multi-valent impurity could strongly upset a self-diffusion experiment due to the very effect this work is examining, a small amount of "improper" aliovalent ions in addition to the $\mathrm{Ca}^{45}$ already deposited was judged to be able to have only a negligible added effect; therefore no further assay was made as to the tracer composition.

The tracer arrived with a specific activity of 17.3 millicuries per mg-Calcium, in a total volume of $.10 \mathrm{~m} \ell$, to which $1.00 \mathrm{~m} \ell$ of $.53 \mathrm{~N}-\mathrm{HC} \ell$ 
was added, forming the basic "stock" solution which provided tracer for all the samples in this experiment. If the original volume was incorrect by even $20 \%$, the "stock" solution volume would be off by only $2 \%$. A portion of this "stock" was taken and further diluted; a portion of this weaker solution was then drawn-off with a pipette of of $.001 \mathrm{~m} \ell$ graduations. A measured volume, accurate to $\pm 1 \%$, was placed onto a piece of "ScotchMagic"-brand tape, and dried under gentle heat. This was then overlaid with $\frac{1}{2}$-mil (.0005") mylar, mounted as if it were a crystal section, and the radioactivity counted. In this manner, one standard ccrrection is made for detector and sample geometry, and for detector efficiency (including the setting of an energy "window" to reject high energy and lowenergy or noise pulses). Since two independent counting systems were used, the same "standard" could independently calibrate both systems. The uncertainty in this standardization is about $\pm 1.5 \%$, and we found the counting systems to be $(11.2 \pm .2) \%$ and $(11.8 \pm .2) \%$ efficient, respectively.

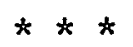

C. Vacuum Deposition Assembly

The vacuum evaporator setup used in this work was approximately 2"diameter topped with a 9"-Vycor "bell-jar", capable of reaching $\sim 10^{-5}$ Torr pressure, affixed with both a thermocouple-pressure-gauge and an ion-gauge, and a nitrogen cold-trap below the evaporator to prevent radioactive atoms from contaminating the pumping system.

The electrodes had screw-holds to mount foils for evaporation, and the entire electrode assembly was replaced with machined pure-copper parts after an early test run at very high foil temperatures (see Appendix VI) welded the foils to the then brass electrodes. This also eliminated the possibility of zinc contamination (due to brass decomposition). 
The foil was made from 2 mil Tantalum sheet, which was cut into uniformly wide rectangular segments, thus providing a uniform heating cross-section. Holes were punched in the ends for mounting in the evaporator. A small dimple was put in the foil midway between the sides, for tracer depositing. Two $45^{\circ}$ bends near the foil ends created a rightangle U-shaped foll. The positioning of the "bends" allowed variation of the dimple to foil-holder distance, and the dimple was thus placed in the geometrical center of the evaporator.

Prior to use, the foil was degreased with acetone and alcohol, rinsed, dipped in concentrated $\mathrm{HCl}$, washed in deionized water, then heated to brilliant white under vacuum for final cleaning.

A test was also run on a blank foil, using different pre-set currents, and heating for a specified time (usually $\sim 30 \mathrm{sec}$ ), while monitoring the pressure. This determined the critical current for out-gassing of the foil, so that a pressure-rise due to residual out-gassing would not be mistaken for tracer evaporation. The blank foil runs also consistently showed a high-current pressure-increase which we associated with incipient in vacuum foil burn-off, providing an approximate upper current bound: A polyhedral mask of approximately equi-radial distance from the evaporator center, designed by Dr. J.L. Mitchell, was used to hold crystals in the evaporator. It was constructed of 5 mil stainless-steel shimstock with 13 punched holes for masking, each $6 \mathrm{~mm}$ in diameter. To this, we spot-welded 4 shimstock "legs" which were then attached with small screws to a brass retaining ring which provided rigid support, as well as making the mask system bottom-heavy so it would not tend to tip over. A second notched brass ring was placed inside the evaporator, providing a consistent mask orientation as well as a support level for the bell-jar top. 
The mask was punched with satellite pin-holes through which we threaded strands, of thin Chromel-P (thermocouple) wire. These could be bent into loops for crystal supports, and, since each wire extended over many loops, the bends provided rigidity without need for either soldering or spot-welding.

$$
* * *
$$

D. Syringe Assembly

Since the fundamental c.d.d. experiment requires sets of crystals with nearly equal amounts of tracer, having arranged the crystal holding mask to be of constant orientation, and having arranged the dimple in the foil to be the center of the evaporator; nearly equal amounts of tracer must be used for each evaporation.

A syringe assembly was constructed consisting of a Teflon syringe holder with an aluminum screw-feed machined to match the plunger of a 1 cc sterile tuberculin syringe. The plunger had a rubber air-tight tip which neatly fitted onto the screw feed. To the tip, the needleless polyethylene syringe was fitted an additional drawn quartz "needle"-dropper which was epoxied onto the syringe from the outside. It, by a small rotation of the screw feed, was capable of creating a small droplet of volume $\leqslant .005 \mathrm{ml}$ (1/6 "normal" drop size).

This smaller assembly fit into a larger array consisting of a few clamps, large brass nuts, and a threaded rod. This arrangement allowed controlled vertical motion of the syringe. A small $2^{\prime \prime} \times 3^{\prime \prime}$ mirror inclined at about $45^{\circ}$, used with a magnifying telescope, allowed careful alignment of the assembly as well as a close-up view of the tracer drying process, from an arm's length away.

$$
\star * *
$$




\section{E. Tracer Deposition}

Four practically identical evaporations onto a total of 52 crystals were performed as follows: Equal measures ( $\pm 1 \%)$ of "stock" tracer solution were drawn out into the quartz-tipped syringe. The syringe was then aligned carefully above the "dimple" of a cleaned tantalum foil. The "dimple" volume was $2.005 \mathrm{ml}$, allowing a "point" source of tracer. A small droplet was formed on the syringe needle, the syringe was slowly lowered to the foil (a clamp was placed on the vertical assembly so the needle could not "crash" into the foil and break), and a very light tap allowed the droplet to make contact with the foil, at which point surface tension pulled it off the syringe. The syringe was raised a bit, and the droplet heated under very gentle current to speed evaporation. By carefully watching the process through the magnifying telescope, we could insure the droplet (which became more concentrated in tracer as the deposition proceeded) did not boil; a possible accident which would have spewed tracer into the ambient environment was thus meticulously avoided. By pre-testing with non-radioactive salt-solution droplets, we found the critical current below which boiling did not ensue, and a lower setting was used throughout the deposition. The final tracer source, after these droplets were dried ( 60 droplets used per foil); was $\simeq 1 / 8^{\prime \prime}$ in circumference due to surface tension slowly spreading out each droplet as it dried. Had larger droplets been used, the resultant area would have been proportionately larger.

This deposition was done in atmosphere, but a light-weight plastic collar attached to the glove bag was put around the evaporation prior to the drying process. After completion, a cover was taped on, the evaporator was weakly evacuated and nitrogen backfilled. The evaporator was then 
re-opened inside the glove bag, which was a nitrogen atmosphere. Crystals were placed on the mask, the evaporator and mask re-assembled, and reevacuated down to $\sim 10^{-4}$ torr.

Each evaporation onto the crystals was done by pre-setting the voltage (current), and switching the current on for 30 seconds, followed by a minute for the pressure to re-equilibrate to its cold-foil value, followed by a small fixed voltage (current) increment, up to the maximum (as blank-foil tests indicated) before foil burn-off ensued. The pressure as a function of time was monitored on an ion-gauge and recorded. Postevaporation counting of the foils showed $298 \%$. of the tracer evaporated off. The entire system was cleaned after each evaporation and a new cleaned foil used. None of the crystals showed coloration on the active face.

A portable $\beta$-detector was put inside the glove bag so that the activity of each crystal could be counted, and crystals of similar activity were grouped together, earmarked for the same temperature diffusion-run. Although crystal activities were matched to $\pm 3 \%$, the variations in crystal thickness resulted in tracer amounts varying within a given temperature run as much as $\pm 10 \%$. This significant deviation had not been realized until after the crystals were sliced and counted, and the total radioactivity then exactly found. This deviation, by itself, introduces a $\pm 10 \%$ variation in the non-linearity parameter ' $\alpha$ ' in the $D(C)$ function:

$$
D(C)=D_{0}\left(1+\alpha C+\beta C^{2}+\ldots\right) \text {. }
$$

After-the-fact, we suggest here a simple procedure to avoid this differential height problem: Half-mil mylar can be mounted over a hole punched in a thin aluminum planchette. The crystal can be placed face down on the mylar, and 
a tare with a screw-feed movable bottom can be inverted over the crystal. By lowering the "bottom" onto the crystal and then re-inverting the whole tare-crystal-mylar assembly, one has corrected for crystal thickness. When the tare assembly is placed into the detector, each active surface will then be a uniform distance away from the source.

$\star * *$

\section{F. Quartz Preparation and Encapsulation}

Quartz ampoules for the crystals were created in the following manner: $12 \mathrm{~mm}$ ID(14mm OD) quartz tubing was necked-down and sealed-off, each such operation creating two "tubes" whose bottoms could be heated and worked to almost flat. These were made, in $\simeq \frac{1}{2}$ and $\simeq 1 \frac{1}{2}$ lengths using a carbide cut-off saw. Some $\frac{1}{2}$ lengths were used as plugs, i.e., they were sealed to the inside of a $15 \mathrm{~mm}$ ID $(17 \mathrm{~mm}$ OD) tube under slightly less than atmospheric pressure. This created a bottom to the $15 \mathrm{~mm}$-wide ampoules quicker than if the large tube were sealed off directly. Other $\frac{1}{2}$ and $1 \frac{1}{2} \cdot$ lengths were used as sample holders.

After a set of "bottoms" were sealed to the larger tubes (maintaining a total length of $\left.9^{\prime \prime}-12^{\prime \prime}\right)$ and leak tested, and after a set of $\frac{1 "}{2}$ sample holders were made, each was thoroughly scrubbed with detergent, soaked in concentrated HCl, rinsed with deionized water several times, and then baked in atmosphere overnight at $\approx 900^{\circ} \mathrm{C}$. This thoroughly oxidized any trace impurities.

One detail of quartz-working worth mentioning is as follows: When necking-down a quartz tube, if the necking area is too long, the torch may only be able partially to heat the tube, resulting in only local melting and a very long seal-off time. On the other hand, too short a neck resulted 
in a gradual thickening of the wall in the neck region, even though the total diameter decreased; it also resulted in a long seal-off time. Using a medium size jeweler's oxygen-hydrogen torch (flame $\approx 3^{\prime \prime}$ ), a $\frac{3^{\prime \prime}}{4}-1^{\prime \prime}$ 'neck' region worked we11.

After the evaporations were complete, two crystals were put in each ampoule, the bottom one in a $\frac{1 "}{2}$-holder, the top one in a $\frac{3^{\prime \prime}}{2}$-holder, capped with a degreased labelled rubber stopper. As a cross-check, each ampoule was numbered directly on the quartz with a diamond scribe. The set was then removed from the glove bag and taken to be sealed off.

Each ampoule was twice evacuated and backfilled with argon, dried using an alcohol-dry ice moisture trap, re-evacuated, and then filled with $1 / 5$ to $1 / 3$ atmosphere of argon. The 9"-12": 1ength was required so that the seal-off process did not over-heat the vacuum hose. Care was also taken to not heat the crystals at this stage. Each ampoule was mounted on a "quick-connect" which allowed reasonably easy axial rotation of the ampoule during seal-off. The outside of the quartz tube was immersed in an ice-water bath and the seal-off was done $\simeq 1 "$ above the water-line. The crystals were maintained below the water-line and thus did not heat up any amount. No attempt was made to isolate the upper and lower crystals, since both were the same tracer and equivalent concentrations. The finished ampoule was cut-off at $\simeq 2 \frac{1}{2}$ " with a carbide saw, and a small "handle" of solid quartz sealed onto the top (again using an ice-water bath).

***

\section{G. Furnace Control and Temperature}

Diffusion anneals were done in an Astro A122 Tubular Furnace with 2 in. ID $\times 12$ in. OD $\times 11$ in. heated length with shunt taps, in which a 
cylindrical nichrome block with three symmetric $1 / 2$ in. diameter $\times 3$ in. sample and 3 thermocouple holes had been drilled. One of the three spaces held a dumny sample, designed by Dr. J.L. Mitchell, which consisted of a Pt $-10 \%$ Rh thermocouple mounted in a NaCl crystal inside a quartz ampoule. We calibrated this thermocouple against a 'standard' thermocouple, and it gave approximately:

$$
\Delta T^{\prime}=.352(T / 100)-.313
$$

where $\mathrm{T}$ =measured temperature $\left({ }^{\circ} \mathrm{C}\right)$ and $\mathrm{T}\left({ }^{\circ} \mathrm{C}\right.$, actua 1$)=\mathrm{T}\left({ }^{\circ} \mathrm{C}\right.$, measured $)+\Delta \mathrm{T}$.

The temperature was controlled by a Thermac Controller governing a R.I. Phase Power Controller. The Thermac Controller was equipped with three modes: Standard, Differential, and Integral; where the controlling output varied with the deviation, derivitive of the deviation, and the integrated deviation respectively. In 'Standard' mode the temperature consistently settled in $\sim 2-3$ degrees away from set-point, with a gradual drift even further away over $\sim \$ 2$ hours. Thus, the system was operated at 'Standard' with (a minimum of) 'Integral'-mode. This made the system slightly unstable, whereby changing the cold junction from one ice-bath to another introduced enough of a 'spike' to cause a resettling a fraction of a degree away. Occasionally, $a \simeq 1^{\circ} \mathrm{C}$ sudden deviation (which, however, returned to its previous temperature) lasting $\sim 10$ minutes was observed in the overnight runs, and these two effects are primarily responsible for an estimated $\pm: 5^{\circ} \mathrm{C}$ uncertainty in temperature throughout this experiment. The thermocouple voltage was monitored on Dana Digital Voltmeter, as well as by using a pre-set "bucking" voltage on a Leeds and Northrup K3 Potentiometer; the voltage difference between the thermocouple and the pre-set value was then amplified by a Leeds and Northrup 9898 D.C. Null 
Detector, which was then used to drive a Mosely chart recorder set to $\simeq 3^{\circ} \mathrm{C}$ full-scale.

Since there were only two free sample spaces and the experiment planned to use 4 times, it was decided to put each sample in the furnace, one at a time, allowing for temperature re-equilibration after each sample was removed. It was judged that samples could be given the closest possible "identical heating history" in this manner, without upsetting any samples in mid-stream diffusion by opening or closing the furnace.

The warm-up curves for each sample at a given temperature were a11 very alike, reaching similar temperatures similar amounts of time after the initial input. It usually took $\simeq 30 \mathrm{sec}$. between the time the furnace was initially opened, and the time the cinder blocks, and thermocouples were fully re-positioned. Usually it took $\simeq 15$ minutes to reach the diffusion anneal temperature; based on earlier work, ${ }^{51}$ the last 3 minutes before that point are probably important. Thus, the ad hoc "time-correction"

$$
t *(\text { warm-up corrected })=t(\operatorname{expt} 1 .)-[.072 \text { tu-units=12 min. }]
$$

is used for [ $\left[\mathrm{nC}\right.$ vs. $\left.x^{2} / t^{*}\right]$-graphing, but (see Chapter 1 , Sec. III) ' $t *$ ' is not used in any analyses.

Finally, a Chromel-P chain was attached to the ampoules for aiding placing in and removing from the furnace. Ampoules were immediately quenched in an ice-water bath after diffusion anneal.

$$
* * *
$$

\section{H. Crystal Sectioning}

Before slicing, each crystal was autoradiographed by placing an $x$-ray film physically atop the crystal for $\simeq 15$ minutes, usually with a 
weight on top to assure good film-crystal contact. An x-ray film holder was made of rubberized black cloth on one side, and $\frac{1}{2} \operatorname{mil}$ mylar followed by black paper on the other; taped together. The film was put emulsion side toward the mylar and backed with 1/16" lucite sheeting for rigidity. Most crystals showed a fairly uniform distribution of tracer, but of ten they had a few "speckles" indicating regions of higher radioactivity. This could possibly be a nucleation of calcium salts on the surface, aided by a fast surface diffusion. But as a surface phenomenon, it should not extend deeply. into the crystal bulk. The other possibility is that it is some surface "hold-up", indicating a different calcium salt, as for example, Ca0. But then having a slow bulk diffusion, again it should not extend deep into the crystal. No explicit correction was attempted for these "speckles."

After diffusion, $3 / 4$ to $1 \mathrm{~mm}$ was cleaved off each of the 4 sides of the crystals, (using the crystal cleaver constructed earlier), so that surface diffusion would not upset the measurement of the activity of deep penetration slices. This usually resulted in a crystal 5.75 to $6.0 \mathrm{~mm}$ on a side.

The crystal was weighed before slicing and after slicing to determine the total weight loss in slicing, and this along with the nominal depth measured on the microtome, determined the weight per standard $8 \mathrm{~mm}$ thick slice.

Crystals were mounted on a brass tare with Salol $\left[\mathrm{C}_{6} \mathrm{H}_{4} \mathrm{OHCOOC}_{6} \mathrm{H}_{5}\right.$ ] which melts at $\simeq 50^{\circ} \mathrm{C}$, supercools, and disolves in acetone. The crystals were "aged" for at least 1 hour after initial opening of the ampoule, to pick-up some ambient moisture, so that, when slicing was started, the initial powder did not "scatter", but came off in small rolls and curls. 
Crystals were sliced using an American Optical Company Precision 860 Sliding Microtome.

Alignment of the crystal parallel to the plane swept out by the knife blade is important so that the slices are perpendicular to the diffusion direction. A non-radioactive test crystal was mounted on a tare, and microtomed flat. A $1^{\prime \prime}$-square front-surface aluminized plane-parallel mirror was set on the crystal surface. A magnifying telescope with crosshairs and an internal half-silvered $45^{\circ}$ prism was set up also. A cross was mounted on the ceiling fluorescent light, its image passing through the half-silvered prism, horizontally to a commercial mirror inclined at $45^{\circ}$, down onto the parallel flat mirror, back up to the commercial mirror, through the prism to the cross-hairs. Adjustments were made to align the cross-image and the cross-hairs. The test crystal was then removed, and the sample put in its place, topped with the flat mirror. The plane of the crystal (and mirror) was then adjusted till the crosses concurred. This provided a gross adjustment of parallelism. As each crystal, after slicing was complete, was already parallel to the knife-plane, it was left on the microtome. while the next sample was prepared.

For finer adjustments, we still had to rely on careful raising of the tare stage guess-adjustments of the tare orientation, and we used a jewelers loupe $(5 X)$ to examine the blade shadow on the crystal surface. The first slice powder was carefully collected onto a small aluminum foil pan placed touching and partly under the tare; powder was gently nudged off the knife with a 1-hair brush. This powder was weighed on a Cahn Electrobalance capable of 1 gg resolution. The method of collection chosen was this: A $6 \mathrm{~mm}$ diameter hole was punched in a 3/4" wide (commercial) 
aluminum-foil strip. Placed over the hole was Scotch-tape, and this was mounted on a glass slide for rigidity. The powder was nudged off the pan with the 1-hair brush onto the aluminum foil which tended to absorb stray charges. The powder was then easily collected onto the central (tape) region. It was then dissolved with a small droplet of deionized water which was then evaporated off under gentle heat. This gave a slightly adherent spread-out salt which.could be overlaid with $1 / 2$ mil mylar and sealedmounted onto a washer. In such a manner, consistently $>98 \%$ of the powder was successfully mounted. In later samples, the powder was collected every few microns, giving 2 or sometimes 3 powder samples, instead of having one "thick" slice of $\simeq 8 \mu \mathrm{m}$.

The rest of the crystal was sectioned using the Mapother tape method. 27 A small strip of Scotch-tape was pressed to the surface of the crystal before passage of the microtome blade, the dust adhering to the tape. The most difficult part is preventing the possibility of the knife cutting the tape. This is scrupulously avoided if

(1). The crystal is oriented so that the knife first contacts a corner, and

(2). The knife contacts the crystal corner before it contacts the tape.

By holding the tape with one finger, its orientation can be adjusted if it appears the knife touches the tape first. This simple test self-orients to the position the tape should be held for successful microtomy, for each slice.

The crystal slices can be visually examined for irregularities in salt distribution, a homogenous distribution indicating a uniformly thick slice. 
Half-mil mylar was placed on a cleaned rubber mat (1/8" thick) and static electricity held the mylar smooth and in place. It was then taped down. Thus, when the tape with crystal was put on it, one was assured of no air bubblès or crinkles. This could then en masse be overlaid with double-stick tape, again without air bubbles and irregularities. If the tape pieces were placed close together, then by cutting in-between each. tape-piece and pressing down washers on all the even numbered slices first, one has pre-cut, 2 sides of each tape encased sample.. Each was inked with an identifying number and cut out from the rest, creating a flat salt sample totally sealed, and with only $\frac{1}{2}$ mil of mylar (and air) between the sample and the B-detector.

The stage (and tare) were raised in multiple-2 micron shifts instead of one large n-micron shift, as it was found to give more accurate thicknesses that way. 51 For quite a few crystals, after the "last" slice. was taken, we checked the weight of several $8 \mu \mathrm{m}$ powder slices and found the weight varied $\sim 10 \%$ from one group of four $2 \mu \mathrm{m}$ knife-passes to the next. On the whole, the average slice thickness (if nominally $8 \mu \mathrm{m}$ ) is $(8.0 \pm .1) \mu \mathrm{m}$; but the slice to slice thickness variation is greater. The uniformity of thickness of a given slice is excellent, but the actual numerical value is not. This variation was corrected in the data-analysis.

It was found that $4 \mu \mathrm{m}$ slices were possible, in terms of creating a uniformly thick sample; thus many later crystals had a large fraction of the active part sliced using the nominal $4 \mu \mathrm{m}$ thickness. Large slice-toslice variations in activity were expected, but the $4 \mathrm{\mu m}$ slices allowed one to slice average over 8 m without loss of information compared to having sliced $8 \mu \mathrm{m}$ slices with no averaging procedure. 
It was observed that the microtome stage consistently relaxed downward a total of about $2 \mu \mathrm{m}$. in $\approx 10$ minutes with negligible further relaxation thereafter. It was also found that after each $8 \mu \mathrm{m}$ slice, if the knife were immediately re-passed over the crystal, additional powder was collected on the knife, but a much smaller amount, if any, formed on the second re-pass. Thus, each "slice" was defined to be the original slice plus this small powder-addition, which was put directly beside the nominal $8 \mu \mathrm{m}$ crystal on the same tape piece. This procedure also effectively cleaned the blade after each use, giving rise to almost no scatter of dust at anytime during the entire slicing procedure.

The washers used to mount crystals on were Albany Products Company commercial grade Flat Stainless Steel washers, size. 3/8. An early and a later order of these washers had the dimensions:

Type 302: OD 1.00", ID .390", .072" thick Type 18-8: OD 1.00", ID .383"; .062" thick.

The relevant change here is in the thickness, which puts a sample further away from the detector. This was $a \simeq 2-3 \%$ effect and is corrected for in the data analysis.

$$
* * *
$$

\section{Counting}

Two counting systems were used. Both consisted of Ortec B-025-150700 totally depleted 700 micron depletion depth Silicon Surface Barrier Detectors with transmission mounts. The detectors were connected to an

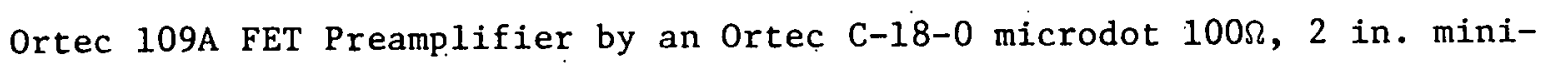
ature cable. An Ortec 114 Preamplifier Power Supply and an Ortec 428 Detector Bias Supply were also used. 
One system for amplifying, analyzing, and counting of the betas was used for the more active slices, and consisted of the detector and preampliffer encased in a "portable" fully electrically isolated aluminum housing; along with an Ortec-490A Amplifier and Single-Channel Analyzer, a Hamner NS-10 Scaler and a Hamner NT-15F-1 Timer/Scaler as a timer. This system had a dead-time of 3.99 microseconds, and an average background of $\simeq .28 \pm .02$ counts $/ \mathrm{sec}$.

The second system used an Ortec $490 \mathrm{~A}$ Amplifier/SCA as an amplifier, and a Tracor-Northern TN-1705 pulse-height analyzer. This system had a dead-time of 9.95 microseconds and a background consistently of $.0415 \pm .0010$ counts/sec., the detector being shielded within a 2 in. thick array of lead bricks; this seçond set-up was used primarily for long-time counting of the inactive deep-penetration slices. An excellent detailed description of this detector system is presented elsewhere. 51

\section{Raw Data}

\section{A. Determining a $\mathrm{C}(\mathrm{x})$-Penetration Profile}

Each profile is ultimately expressed as a set of coefficients $\left\{B_{p}\right\}$ in:

$$
C\left(x, t_{R}\right)=\sum B_{p} H_{2 p}\left(x / x_{R}\right) \exp -\left(x / x_{R}\right)^{2} .
$$

The measured counts/sec of each slice is corrected for each of these effects, in this order:

(1). Dead time

(2). Background

(3). Finite count-time (to allow for a finite amount of decay of the tracer during the course of a long count) 
(4). Beta-absorption in the crystal slice due to its finite thickness

(5). Changes in washers thickness

(6). Counting efficiency (fraction of decays recorded, compared to actual radioactivity in sample)

(7). Tracer radioactivity decay (the time $t=0$ point is taken as the in-furnace time when the diffusion anneal began)

(8). Non-radioactive Ca-atoms (using the $17.3 \mathrm{millicuries} / \mathrm{mg}-\mathrm{Ca}$, one converts from radioactivity to impurity amount)

(9). Area of crystal. (this converts from impurity amount per slice to impurity amount per volume)

To correct for beta-absorption, we assume absorption of betas to go as $\left(\exp -x / \mu_{A}\right)$ and, by taking an active source and overlaying with an additional layer of Scotch tape-crystal-mylar; using non-radioactive crystals of nominal thicknesses of $[0 \mu \mathrm{m}, 2 \mu \mathrm{m}, 4 \mu \mathrm{m}, 8 \mu \mathrm{m}, 16 \mu \mathrm{m}]$ and a few samples at each thickness, we found $\mu_{\mathrm{A}} \simeq 46.5$ microns.

$$
* * *
$$

Many of the crystals, especially the shorter-time runs, were initially sliced into $4 \mu$ samples, and, although this procedure introduces larger slice-by-slice deviations, it does "pin" the profile with twice as many data points. This was done because we were capable of creating uniformly thick $4 \mu$ samples and because a thicker slice will tend to be followed by a thinner one, and the orthogonal function procedure, being unable to change so rapidly, will seek out the average. The data "noise" was also reduced by the following 2 stage-process:

(1). Slice averaging: Here, each datum $C\left(x_{i}\right)$ is replaced by 


$$
2 \bar{C}\left(x_{i}\right)=C\left(x_{i}\right)+.5\left[C\left(x_{i-1}\right)+c\left(x_{i+1}\right)\right]
$$

(2). Reducing "local" noise: A rough $\mathrm{N}$-polynomial fit is generated to the data. Plotting $\left[C\left(x_{i}\right.\right.$, curve-fit value $) / C\left(x_{i}\right.$, data value $\left.)-1\right]$ vs. $\left(x_{i}\right)$; equivalent percentage deviations in slice-thickness will appear as equivalent size deviations. Groups of approximately the same deviation are taken together, an average deviation computed, and each slice thickness in the group is corrected by this same factor, the concentration then altered to preserve $[c \Delta x]$. (which, like mass, is conserved). This changes the penetration depth of each slice, and after that adjustment is made, the smoothed data are expressed as a new polynomial series.

We set up a program to allow each stage of corrections only once, as repeated application of these corrections obviously. spuriously converges upon zero error. This procedure, furthermore, can only minimally affect. the profile shape, since it is composed of entirely "local" changes, which operate on the scale of $\approx 8 \mu \mathrm{m}$, and its primary effect was to allow a $4-6$ polynomial fit to match the "tail" region on a [ $\mathrm{lnC}$ vs. $\left.\mathrm{x}^{2}\right]$-graph (even though, as noted before, the Hermite polynomial fitting procedure inherently minimizes error on a [C vs. $x$ ]-graph). The smoothed data and the fit consistently matched to better than $2 \%$.

$$
* * *
$$

\section{B. Error Analysis}

Errors in determination of the parameters $\left(D_{0}, \alpha, \beta\right)$ fall into two broad categories.

One type is due to errors in the kinematical variables (i.e., temperature, diffusion time, penetration depth) which affect the 
determination of $\mathrm{D}_{\mathrm{O}}$ directly or via affecting $\mathrm{x}_{\mathrm{N}}^{2}$ in the expression:

$$
x_{N}^{2}=x_{R}^{2}+4 D_{O}\left(t_{F}-t_{R}\right)
$$

which is used in an exponent in the minimizing function (recall $\Phi^{2}$ has a propagated-state term $\left.\hat{C}_{R}\left(x, t_{F}\right) \sim \exp -\left(x / x_{N}\right)^{2}\right)$.

Even though the warm-up time correction is not a factor in determining $D_{0}$ in the method developed here, the fact that samples were not given identical heating histories at a given temperature will affect $\left(t_{F}-t_{R}\right)$. Since a full warm-up time correction is $\approx 3$. minutes, we estimate that $\left(t_{F}-t_{R}\right)$ is accurate to $\leqslant l$ min., which is a small correction to practically every diffusion run except the shortest time and higher temperatures, where it is a $2 \%$ correction. On all other runs, it is a $\leqslant 1 \%$ correction.

Since the powder-slice thicknesses are weight-determined and their total weight characteristically is less or equivalent to an $8 \mu \mathrm{m}$ slice, and since a fair fraction of the weight comes from powder accumulated from aligning the crystal parallel to the knife, we estimate the uncertainty in the $x=0$ position as $\pm 2 \mu \mathrm{m}$. Since the $x_{R}$-value for the different datasets, ranged from $\sim 8-15$ xu-units $(90-150 \mu \mathrm{m})$, using $\mathrm{D}_{0} i x^{2} / t$, this is at most a $3-5 \%$ correction.

The random deviations between the continuous datafit and the discrete data, which amounted to less than $2 \%$; as well as the counting statistical error of $1 \%$ (as each sample was counted for at least $10^{4}$ counts) both contribute negligibly to errors in $\mathrm{D}_{0}$.

The furnace temperature was controlled to $\pm .5^{\circ} \mathrm{C}$ which gives an additional $1.5 \%$ in $D_{0}$. The temperature gradient in the furnace was negligible. 51 
The total error in determining $D_{0}$ is about $5 \%$, possibly as high as $7 \%$ for a short-time run.

The second type of error is associated with normalization; i.e., $\int \tilde{C}\left(x, t_{i}\right) d x=N(i):$ This primarily affects the non-linearity parameter ' $\alpha$ ' (see Chapter 4, Sec. II). There is a $1 \%$ error in the "counting efficiency," as noted earlier. The actual values of $N(i)$, total impurity amount, are very well determined, since great care was taken to collect and preserve radioactivity amount. Table $\langle 7.1>$ shows the $N(i)$ values, as well as the normed profiles at $x=0$ (i.e., $c(x=0)$ where $\left.\int c\left(x, t_{i}\right) d x=\hat{1}\right)$ and the $x_{R}$-expansion parameters which were all chosen in this work to obey:

$$
\text { (apparent) L.S. (limiting-slope) } \equiv-1 / \mathrm{x}_{\mathrm{R}}{ }^{2} \cdot
$$

When correlating two datasets (see Eqs. (8.04) to (8.05)), an average normalization is used:

$$
N(i, j)=. .5[N(i)+N(j)]
$$

and as the $N(i)$-values for different crystals differ by $\sim 20 \%$, this introduces an uncertainty in $\alpha$ of $\pm 10 \%$. Finally, we estimate that the nonuniformity of tracer deposition. ("speckles") might add a few percent further uncertainty in ' $\alpha$ '. Thus, the total error in determining ' $\alpha$ ' is about $\pm 15 \%$.

Finally, reviewing the $N(i)$ values in Table $\langle 7.1\rangle$, we note that the datasets $(\mathrm{Q} 3, \mathrm{~T} 2, \mathrm{U} 1, \mathrm{U} 2)$ have normalizations higher than average (i.e., >6.0) and (R4) has a normalization significantly lower than average (i.e., <4.5). Since both crystals were sliced for the shortest two times of the highest temperature, the (U1A, U2A) crystal results were used for the analysis since their normalizations are within the $N(i)=(4.5,6.0)$ 
TABLE 7.1

\begin{tabular}{|c|c|c|c|c|c|c|c|c|}
\hline [IATASE'T & TEMF' $K$ & TIME & *FOLY & $N(I)$ & $c(x=0)$ & ERR.\% & $X R(t /-)$ & $n x$ \\
\hline 01 & 847.5 & 4.05 & $\hat{1}$ & $\begin{array}{l}5.5614 \\
5.5581\end{array}$ & $\begin{array}{l}.04685 \\
.04955\end{array}$ & $\begin{array}{l}3.520 \\
1.839\end{array}$ & 9.142 & .287 \\
\hline Q2 & 847.5 & 8.01 & $\begin{array}{l}4 \\
7\end{array}$ & $\begin{array}{l}5.4451 \\
5.4387\end{array}$ & $\begin{array}{l}.03470 \\
.03485\end{array}$ & $\begin{array}{l}1.557 \\
1.376\end{array}$ & 12.833 & .262 \\
\hline Q3 & 847.5 & 15.93 & $\begin{array}{l}4 \\
7\end{array}$ & $\begin{array}{l}6.41 .47 \\
6.4088\end{array}$ & $\begin{array}{l}.02632 \\
.02683\end{array}$ & $\begin{array}{l}2.333 \\
1.990\end{array}$ & 15.289 & .172 \\
\hline Q4 & 847.9 & 31.77 & 4 & $\begin{array}{l}4.8716 \\
4.8829\end{array}$ & $\begin{array}{l}.02129 \\
.02172\end{array}$ & $\begin{array}{l}3.187 \\
2.019\end{array}$ & 22.723 & .333 \\
\hline Fil & 897.2 & 1.638 & $\begin{array}{l}4 \\
6\end{array}$ & $\begin{array}{l}4.7985 \\
4.7989\end{array}$ & $\begin{array}{l}.05683 \\
.05602\end{array}$ & $\begin{array}{l}1.858 \\
2.160\end{array}$ & 8.530 & .191 \\
\hline 122 & 897.7 & 3.150 & 6 & $\begin{array}{l}5.3432 \\
5.3341\end{array}$ & $\begin{array}{l}.04045 \\
.04339\end{array}$ & $\begin{array}{l}4.908 \\
1.382\end{array}$ & 9.618 & .256 \\
\hline Fi3 & 898.0 & 6.21 & $\begin{array}{l}4 \\
6\end{array}$ & $\begin{array}{r}4.5966 \\
4.5913\end{array}$ & $\begin{array}{l}.03303 \\
.03454\end{array}$ & $\begin{array}{l}3.295 \\
0.8367\end{array}$ & 11.874 & .266 \\
\hline Ri4 & 898.2 & 12.24 & $\begin{array}{l}4 \\
6\end{array}$ & $\begin{array}{r}3.59506 \\
3.59512\end{array}$ & $\begin{array}{l}.02972 \\
.02967\end{array}$ & $\begin{array}{l}0.7983 \\
0.8105\end{array}$ & 13.609 & .086 \\
\hline $1+5$ & 898.2 & 24.57 & 4 & $\begin{array}{l}4.6190 \\
4.6195\end{array}$ & $\begin{array}{l}.02015 \\
.02006\end{array}$ & $\begin{array}{l}1.305 \\
1.402\end{array}$ & 22.063 & .307 \\
\hline .51 & 948.0 & 0.810 & $\begin{array}{l}4 \\
6\end{array}$ & $\begin{array}{l}5.3746 \\
5.3747\end{array}$ & $\begin{array}{l}.06245 \\
.06213\end{array}$ & $\begin{array}{l}2.701 \\
2.760\end{array}$ & 8.934 & .104 \\
\hline 52 & 947.1 & 1.530 & $\begin{array}{l}4 \\
6\end{array}$ & $\begin{array}{l}5.0792 \\
5.0782\end{array}$ & $\begin{array}{l}.04885 \\
.04856\end{array}$ & $\begin{array}{l}1.616 \\
1.680\end{array}$ & 9.192 & .030 \\
\hline 53 & 946.8 & 2.97 & $\begin{array}{l}4 \\
6\end{array}$ & $\begin{array}{l}5.2174 \\
5.2133\end{array}$ & $\begin{array}{l}.03504 \\
.03487\end{array}$ & $\begin{array}{l}1.700 \\
1.772\end{array}$ & 12.002 & .178 \\
\hline 54 & 947.6 & 5.85 & $\begin{array}{l}4 \\
6\end{array}$ & $\begin{array}{l}5.9935 \\
5.9991\end{array}$ & $\begin{array}{l}.02651 \\
.02626\end{array}$ & $\begin{array}{l}1.738 \\
1.546\end{array}$ & 16.783 & .097 \\
\hline 12 & 997.7. & 0.816 & 4 & $\begin{array}{l}6.7513 \\
6.7577\end{array}$ & $\begin{array}{l}.05033 \\
.04994\end{array}$ & $\begin{array}{l}0.9092 \\
1.086\end{array}$ & 9.792 & .111 \\
\hline T3 & 997.2 & 1.530 & $\begin{array}{l}4 \\
6\end{array}$ & $\begin{array}{l}4.9915 \\
4.9907\end{array}$ & $\begin{array}{l}.03843 \\
.03876\end{array}$ & $\begin{array}{l}1.514 \\
1.230\end{array}$ & 12.880 & .288 \\
\hline T4 & 997.2 & 2.982 & $\begin{array}{l}4 \\
6\end{array}$ & $\begin{array}{l}4.6579 \\
4.6571\end{array}$ & $\begin{array}{r}.02989 \\
.03009\end{array}$ & $\begin{array}{l}1.466 \\
1.315\end{array}$ & 15.919 & .242 \\
\hline U1 & $1048 \cdot 4$ & 0.276 & $\begin{array}{l}3 \\
5\end{array}$ & $\begin{array}{l}6.4374 \\
6.4379\end{array}$ & $\begin{array}{l}.06507 \\
.06504\end{array}$ & $\begin{array}{l}1.558 \\
1.556\end{array}$ & 7.361 & .025 \\
\hline U1A & , & .' & $\begin{array}{l}3 \\
5\end{array}$ & $\begin{array}{l}4.5327 \\
4.5337\end{array}$ & $\begin{array}{l}.06607 \\
.06570\end{array}$ & $\begin{array}{l}1.950 \\
1.997\end{array}$ & 7.730 & .129 \\
\hline $\mathrm{U} 2$ & 1048.0 & 0.456 & $\begin{array}{l}3 \\
5\end{array}$ & $\begin{array}{l}6.9501 \\
6.9500\end{array}$ & $\begin{array}{l}.04893 \\
.04874\end{array}$ & $\begin{array}{l}1.659 \\
1.649\end{array}$ & 10.543 & .013 \\
\hline U2A & & . & $\begin{array}{l}3 \\
5\end{array}$ & $\begin{array}{l}4.6916 \\
4.6914\end{array}$ & $\begin{array}{l}.04979 \\
.04972\end{array}$ & $\begin{array}{l}2.489 \\
2.464\end{array}$ & 10.198 & .113 \\
\hline U3 & 1047.8 & 0.816 & $\begin{array}{l}3 \\
5\end{array}$ & $\begin{array}{l}5.6371 \\
5.6369\end{array}$ & $\begin{array}{l}.04078 \\
.04072\end{array}$ & $\begin{array}{l}1.336 \\
1.341\end{array}$ & 12.982 & .093 \\
\hline USA & . & . & $\begin{array}{l}3 \\
5\end{array}$ & $\begin{array}{l}5.6299 \\
5.6304\end{array}$ & $\begin{array}{l}.04064 \\
.04040\end{array}$ & $\begin{array}{l}1.288 \\
1.180\end{array}$ & 12.651 & .097 \\
\hline U4 & 1048.1 & 1.530 & $\begin{array}{l}3 \\
5\end{array}$ & $\begin{array}{l}5.4074 \\
5.4069\end{array}$ & $\begin{array}{l}.02755 \\
.02779\end{array}$ & $\begin{array}{l}1.745 \\
1.457\end{array}$ & 17.948 & .259 \\
\hline$U 4 A$ & "' & . & $\begin{array}{l}3 \\
5\end{array}$ & $\begin{array}{l}2.92611 \\
2.92607\end{array}$ & $\begin{array}{l}.031718 \\
.031724\end{array}$ & $\begin{array}{l}2.452 \\
2.448\end{array}$ & 18.204 & .390 \\
\hline
\end{tabular}

$N(I)=$ TOTAL 'AREA' UNDER $[C(X)$ US. $X]$-CURUE (NANOMOLES/CM**2) $C(x=0)=$ CONC. AT $X=0$ USING NORMEII FFOFILE ('AKEA' $=1.00)$ EFF. $\%$ = FEF-CENTAGE EFFIOF HETWEEN $C(X$, IIATA $)$ ANII C. $X$, IIATAFIT $)$ $X F=$ EXFANSION FARAMETEF; CHOSEN FFOM LIMITING.SLOFE $=-1 /(X K) * * 2$ $I I X=S T I I$. IIEV. IN 'XF' 
range. Finally, the low total impurity amount of (R4) compared to the (R3, R5) datasets strongly contributes to (R4) having a shallow penetration profile. An ad hoc correction for this is given in the data analysis (see parts of Secs. IV and V, Chapter 8).

$* * *$

\section{Plots of Raw Data}

Graphic presentation of the 'smoothed' raw data, on both [C vs. $x$ ]and [ $\ell n C$ vs. $\dot{x}^{2}$ ]-axes, as well as polynomial (multiplied by a gaussian) fits to the data points, are both given next:

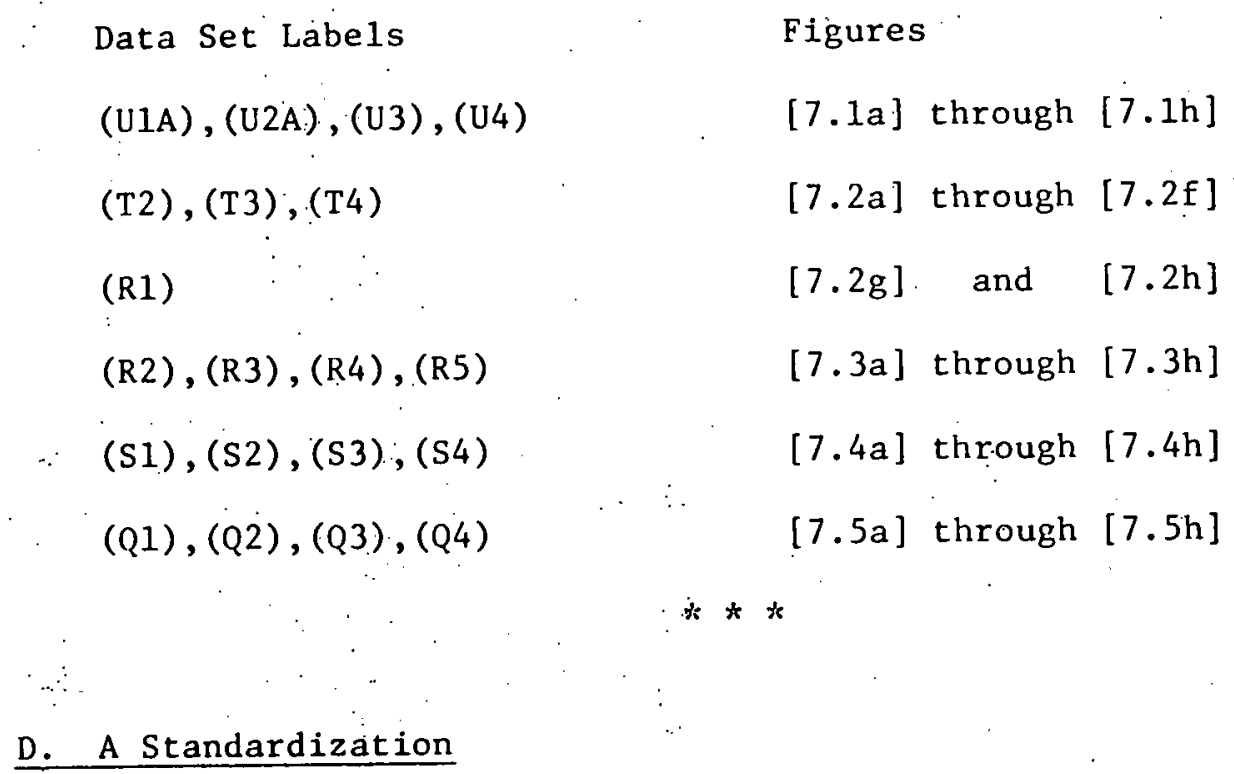

In Figs: [7.6a] through [7.6d], plots are presented for two additional datasets, labelled ' $U 3 A^{\prime}$ ' and ' $U 4 A$ ', both from the highest temperature run. As 'U3A' and sample 'U3' shared the same ampoule, as did 'U4A' and 'U4', each pair has the exact same diffusion time, temperature, and heating history. What can differ between the samples is the total impurity amount initially deposited, pre-diffusion anneal, onto the crystal surfaces. 
This total impurity amount (area under [C vs. $x]$-curve) enters into the determination of the non-linearity parameters (see Chapter 4, Sec. II), and thus, it governs the size of the non-iinearity effect. From. Table $<7.1\rangle$, these samples had "areas" of:

$\begin{array}{cccc}\text { Label } & \text { Total Area } & \text { Label } & \text { Total Area } \\ \text { U3 } & 5.637 & \text { U4 } & 5.407 \\ \text { U3A } & 5.630 & \text { U4A } & 2.926 .\end{array}$

Thus, the datasets ' $\mathrm{U} 3$ ' and 'U3A' should be practically identical, and any difference between them is a measure of the overall resolution of the combined experimental method, and the data-fitting procedure. Figs. [7.7a] and $[7.7 b]$ show, on both [C vs. $x]$-axes and [ $\mathrm{nC}$ vs. $\left.\mathrm{x}^{2}\right]$-axes, a comparison between the. 'U3' and ' $U 3{ }^{\prime}$ ' datasets. One finds that they agree to $0.96 \%$ on the [C vs. $x$ ]-plot, with a tail deviation on a [ lnC vs. $\left.x^{2}\right]$-plot that is probably due to the datafitting procedure being less accurate in that region.

The sample' 'U4', when compared with 'U4A', gives a measure of the concentration dependent diffusion effect, and here the result is striking. For a change of approximately 2 in total impurity amount, even for this longest time, highest temperature diffusion run (where the diffusion should be most 'normal'), there is an $11.6 \%$ deviation between the normed 'U4' profile, and the normed 'U4A' profile (Fig. [7.8a]).

The [ lnC vs. $\left.x^{2}\right]$-plot (Fig. [7.8b]). shows the lower concentration profile as almost gaussian, with the higher concentration profile showing both curvature as well as a deeper average penetration. This clearly shows the possible magnitude of this non-1inear diffusion effect. 
Figures 7.1a through 7.1h. Plots of raw data, 'smoothed' by averaging over regions of width 2 slice-thicknesses, as detailed in text. Highest temperature run; Average temperature $=775^{\circ} \mathrm{C}$. Each dataset is plotted both on [C vs. $x$ ]-axes and [ $\ln C$ vs. $\left.x^{2}\right]$-axes, with a (polynomial) $\times$ gaussian least squares fit also shown (continuous line). Individual data points are labelled by a cross ( + ), with a "tacked-on-tail" (see Chapter 2, Sec. V) on a [C vs. x]-plot denoted by the symbol $(x)$. Each individual dataset is labelled as follows:

Label Diffusion Time $\left(10^{4} \mathrm{sec}.\right)$ Temperature $\left({ }^{\circ} \mathrm{C}\right)$ \#Poly Noise ${ }^{*}(\%)$

$\begin{array}{lrrrr}\text { U1A } & .276 & 775.2 & 3 & 1.95 \\ \text { U2A } & .456 & 774.8 & 5 & 2.46 \\ \text { U3 } & .816 & 774.6 & 3 & 1.34 \\ \text { U4 } & 1.530 & 774.9 & 4 & \simeq 1.5\end{array}$

" "Noise" is the percentage deviation on a [C vs. $\mathrm{x}$ ]-graph between the data points and the polynomial fit.

The lower part of each [C vs. $x$ ]-graph plots the fractional deviation, $\Delta_{1}$, between the data points and the polynomial fit.

$$
\Delta_{1} \equiv 1-C(x, \text { polynomial } \mathrm{fit}) / \mathrm{C}(\mathrm{x} \text {, data point })
$$

The $\left[\Delta_{1}\right.$ vs. $\left.x\right]-p l o t$ has the same horizontal axis as the [C. vs. $x$ ]plot, and the vertical axis has $\Delta_{1}=.10$ corresponding to a $10 \%$ difference between a given data point, and the polynomial fit curve. Equivalent variations in slice thickness plot as equivalent deviations on the $\left[\Delta_{1}\right.$ vs. $\left.x\right]$-graph.

The lower part of each [ lnC vs. $x^{2}$ ]-graph plots $\left[\Delta_{2}\right.$ vs. $x^{2}$ ] where

$$
\Delta_{2} \equiv \ln [C(x, \text { data point }) / C(x, \text { polynomial fit })]
$$

with a vertical axis scale exactly as $\Delta_{1}$, and with a horizontal scale as given on the [lnC vs. $x^{2}$ ]-plot. 

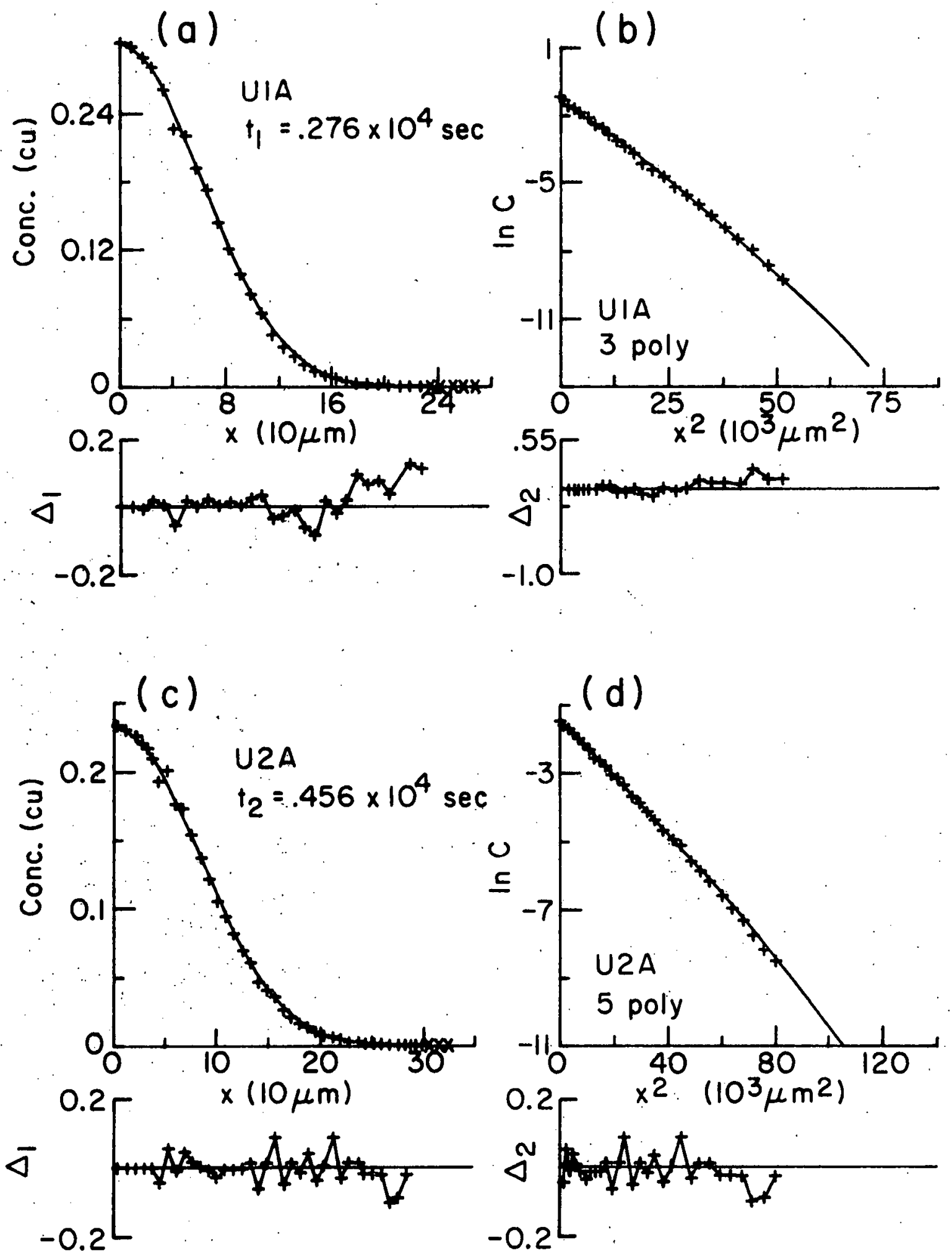

Fig. 7.1 

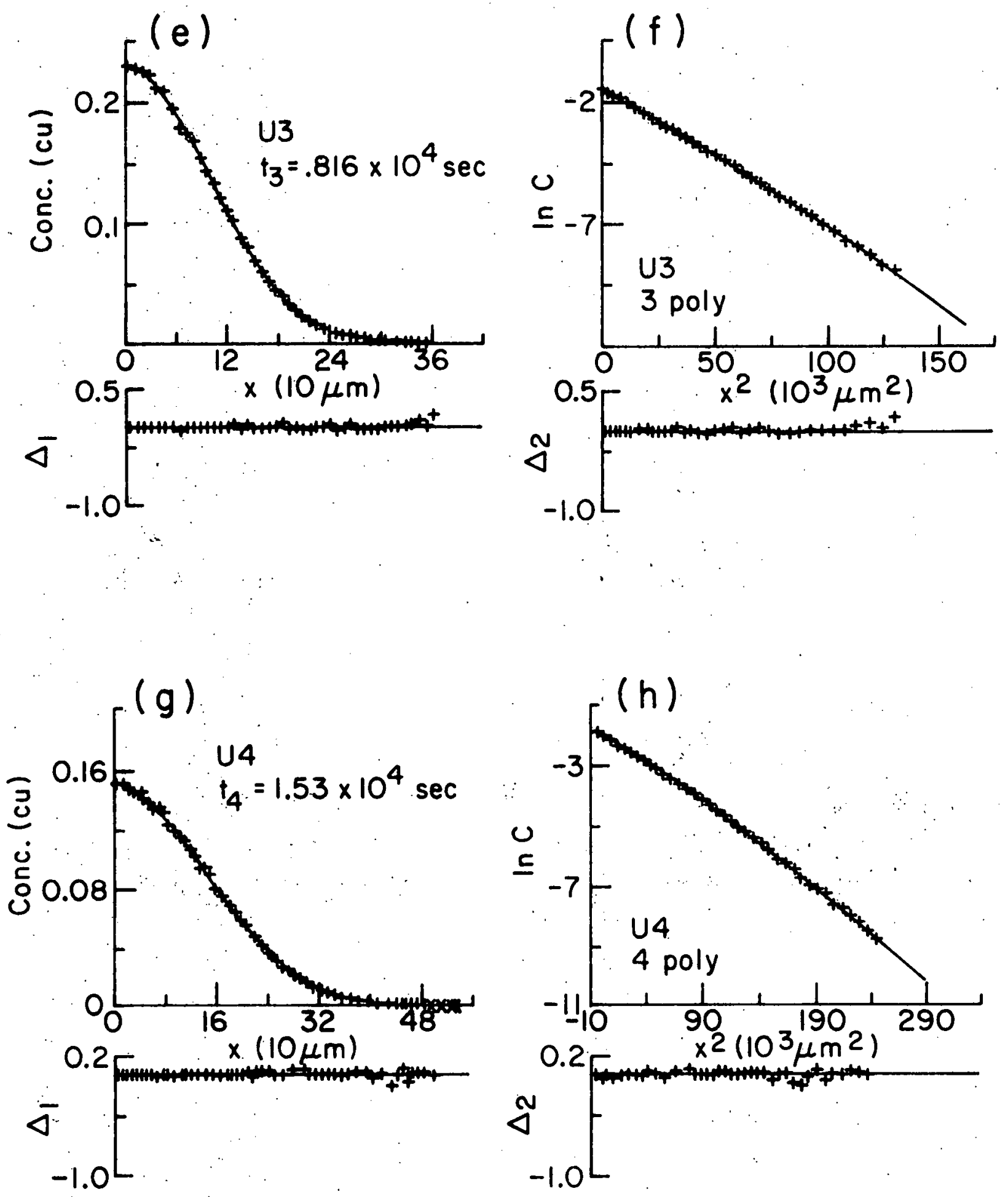

Fig. 7.1 
Figures $7.2 \mathrm{a}$ through $7.2 \mathrm{f}^{+}$' Plots of the 'smoothed' raw data. Next to highest temperature run; Average temperature: $724^{\circ} \mathrm{C}$. The presentation form follows Figure 7.1, with each individual dataset labelled as follows :

Label Diffusion Time $\left(10^{4} \mathrm{sec}.\right)$ Temperature $\left({ }^{\circ} \mathrm{C}\right)$ "\#Poly Noise $(\%)$

$\begin{array}{lllll}\text { T2 } & 0.816 & 724.5 & 4 & 0.91 \\ \text { T3 } & 1.530 & 724.0 & 4 & 1.51 \\ \text { T4 } & 2.98 & 724.0 & 4 & 1.47\end{array}$

" "Noise" refers to the percentage deviation on a [C vs. x]-graph between the data points and the polynomial fit:

$$
\text { Noise }(\%) \equiv 100\left[\sum_{i}\left(C\left(x_{i}, \text { poly.fit }\right)-C\left(x_{i}\right)\right)^{2}\right]^{1 / 2} /\left[\sum_{i}\left(C\left(x_{i}\right)\right)^{2}\right]^{1 / 2}
$$

${ }^{\dagger}$ Figures $7.2 \mathrm{~g}$ and $7.2 \mathrm{~h}$ are part of the next. to lowest temperature run. See Figure 7.3. 

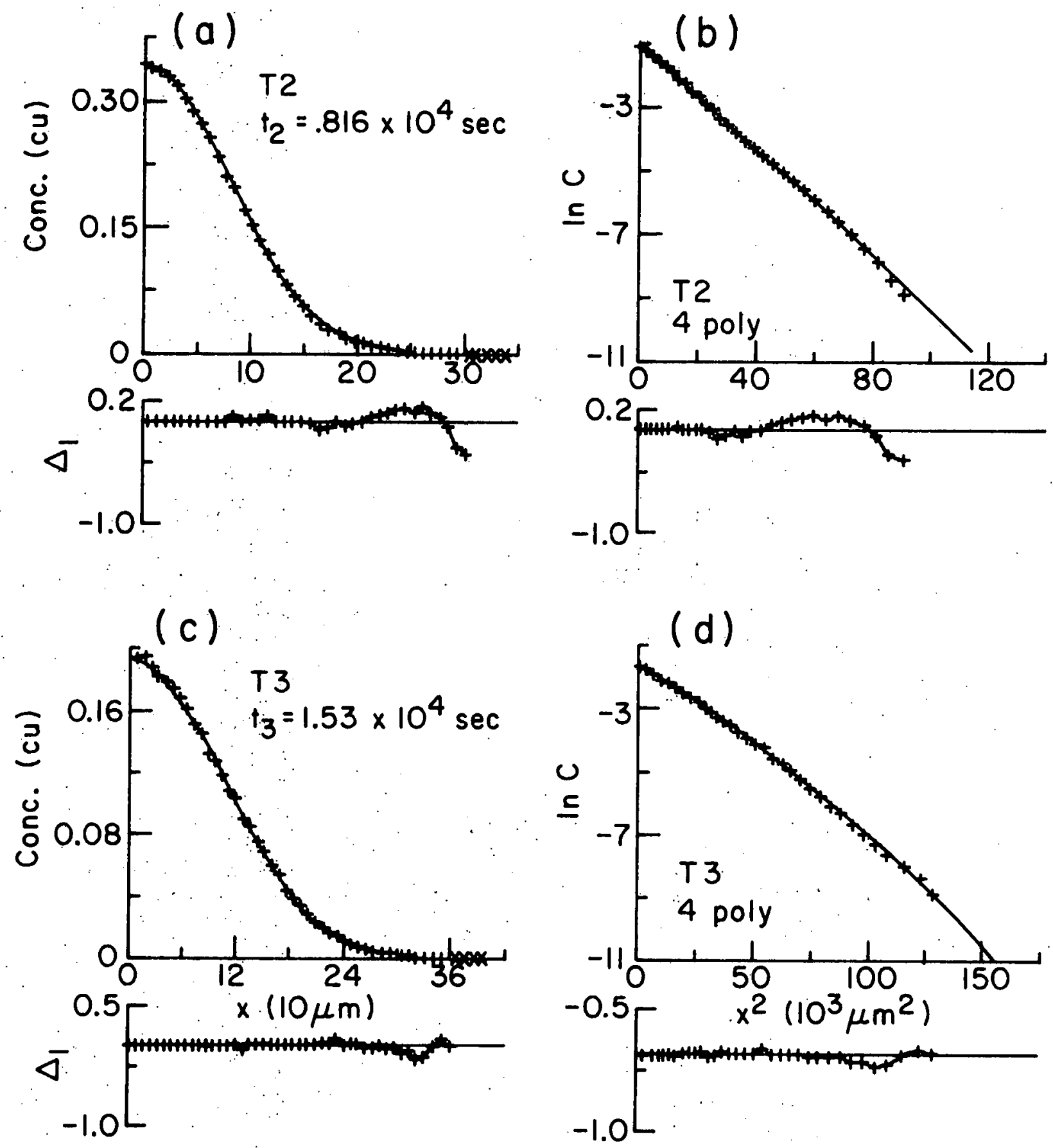

Fig. 7.2 

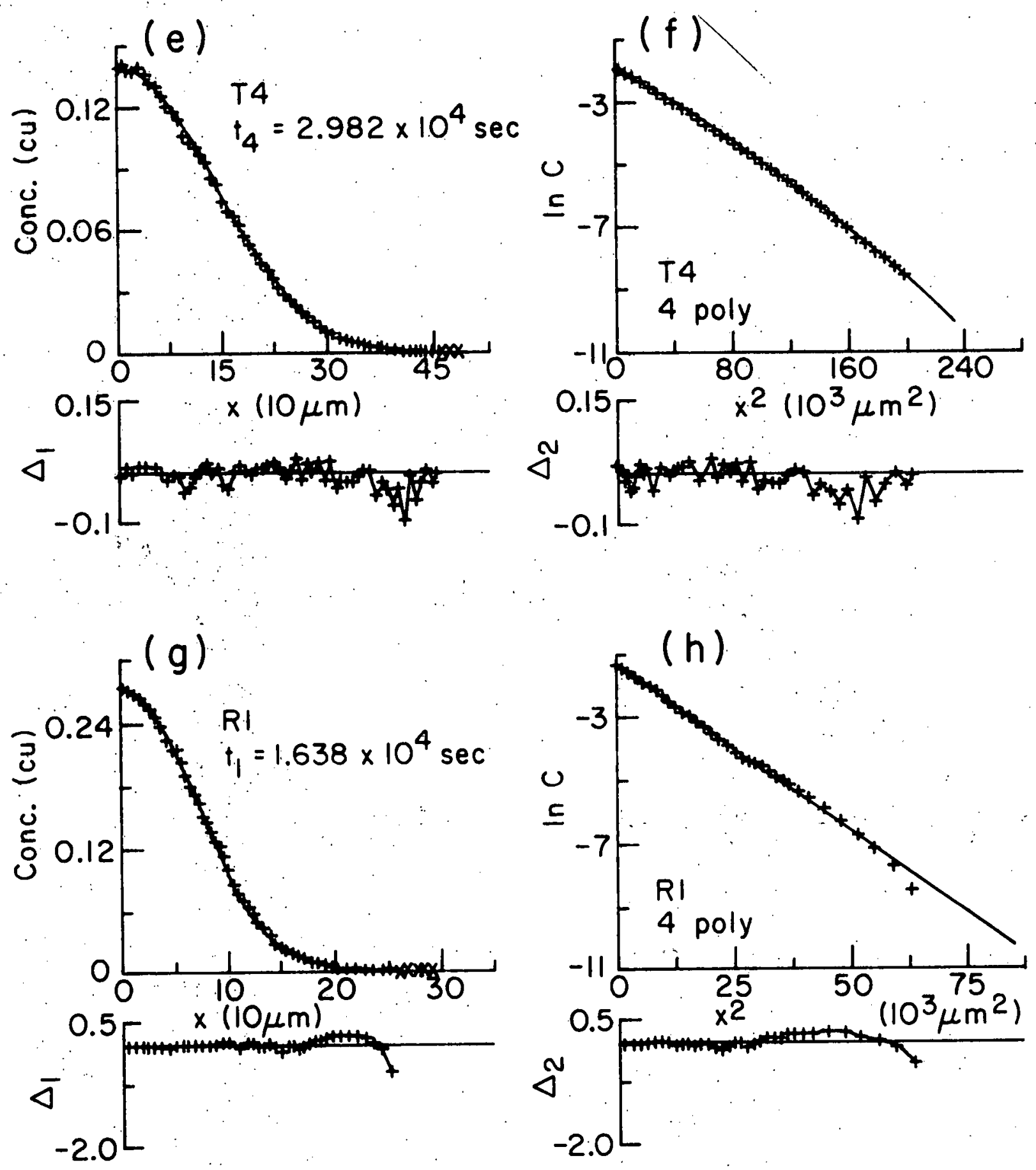

Fig. 7.2 
Figures $7.3 \mathrm{a}$ through $7.3 \mathrm{~h} .^{\dagger}$ Plots of the 'smoothed' raw data. Next to lowest temperature run; Average temperature: $625^{\circ} \mathrm{C}$. The presentation form.follows Figure 7.1, with each individual dataset labelled as follows:

Labe1. Diffusion Time $\left(10^{4}\right.$ sec.) Temperature $\left({ }^{\circ} \mathrm{C}\right)$ \#Poly. Noise (\%)

$\begin{array}{lllll}\text { R1 } & 1.638 & 624.0 & 4 & 1.89 \\ \text { R2 } & 3.15 & 624.5 & 6 & 1.38 \\ \text { R3 } & \therefore .21 & 624.8 & 6 & 0.84 \\ \text { R4 } & 12.24 & 625.0 & 4 & 0.80 \\ \text { R5 } & 24.57 & 625.0 & 4 & 1.31\end{array}$

${ }^{\dagger}$ See also Figure 7.2 : 
154
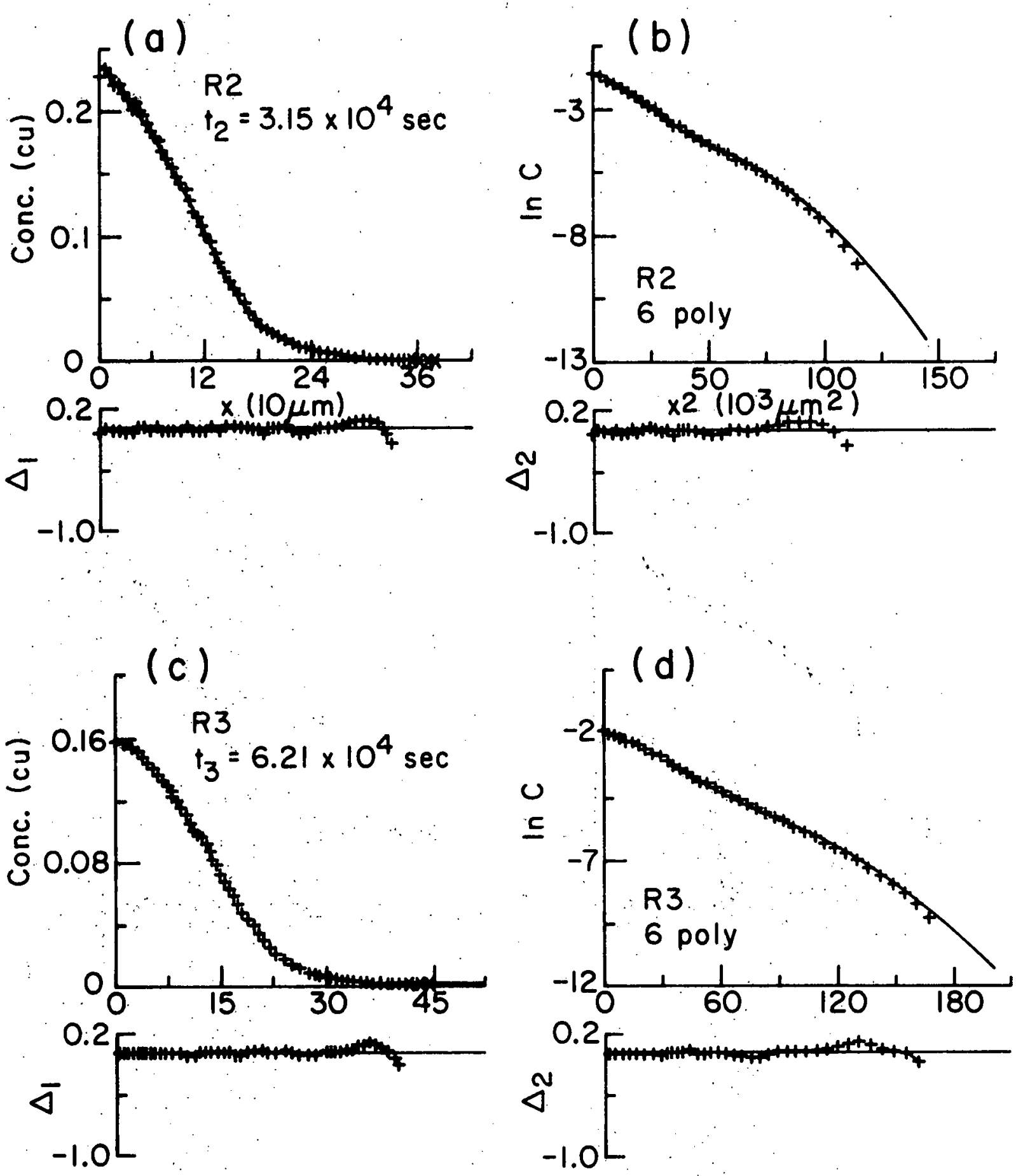

Fig. 7.3 
Figures $7.4 \mathrm{a}$ through $7.4 \mathrm{~h}$. Plots of the 'smoothed' raw data. Median temperature run; Average temperature: $674^{\circ} \mathrm{C}$. The presentation form follows Figure 7.1, with each individual dataset labelled as follows:

$\begin{array}{ccccc}\text { Labe1 Diffusion Time }\left(10^{4} \text { sec.) }\right. & \text { Temperature }\left({ }^{\circ} \mathrm{C}\right) & \text { \#Poly } & \text { Noise (\%) } \\ \text { S1 } & 0.810 & 674.8 & 4 & 2.70 \\ \text { S2 } & 1.530 & 673.9 & 4 & 1.62 \\ \text { S3 } & 2.97 & 673.6 & 4 & 1.70 \\ \text { S4 } & 5.85 & 674.4 & 4 & 1.74\end{array}$


155
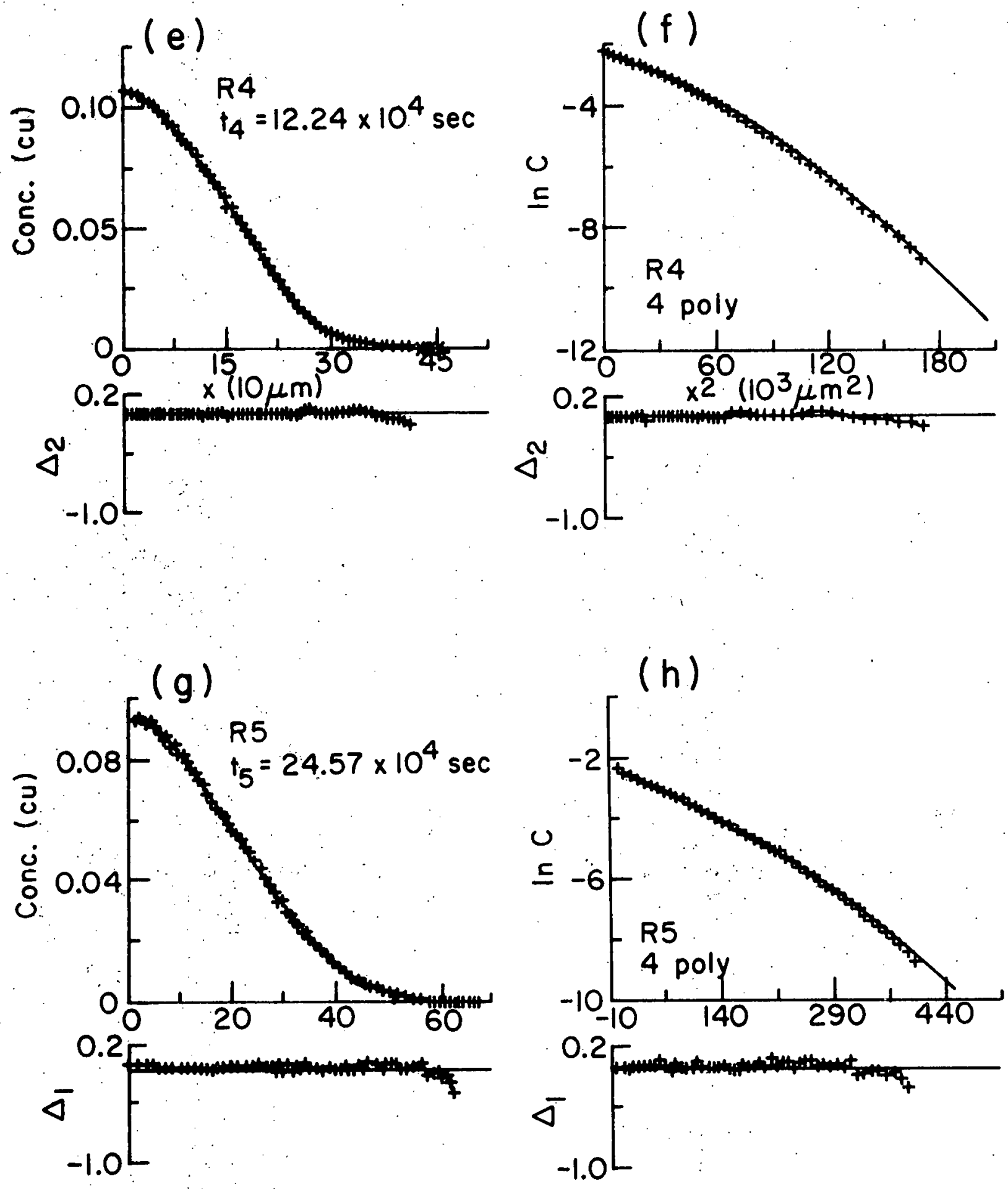

Fig. 7.3 
157.
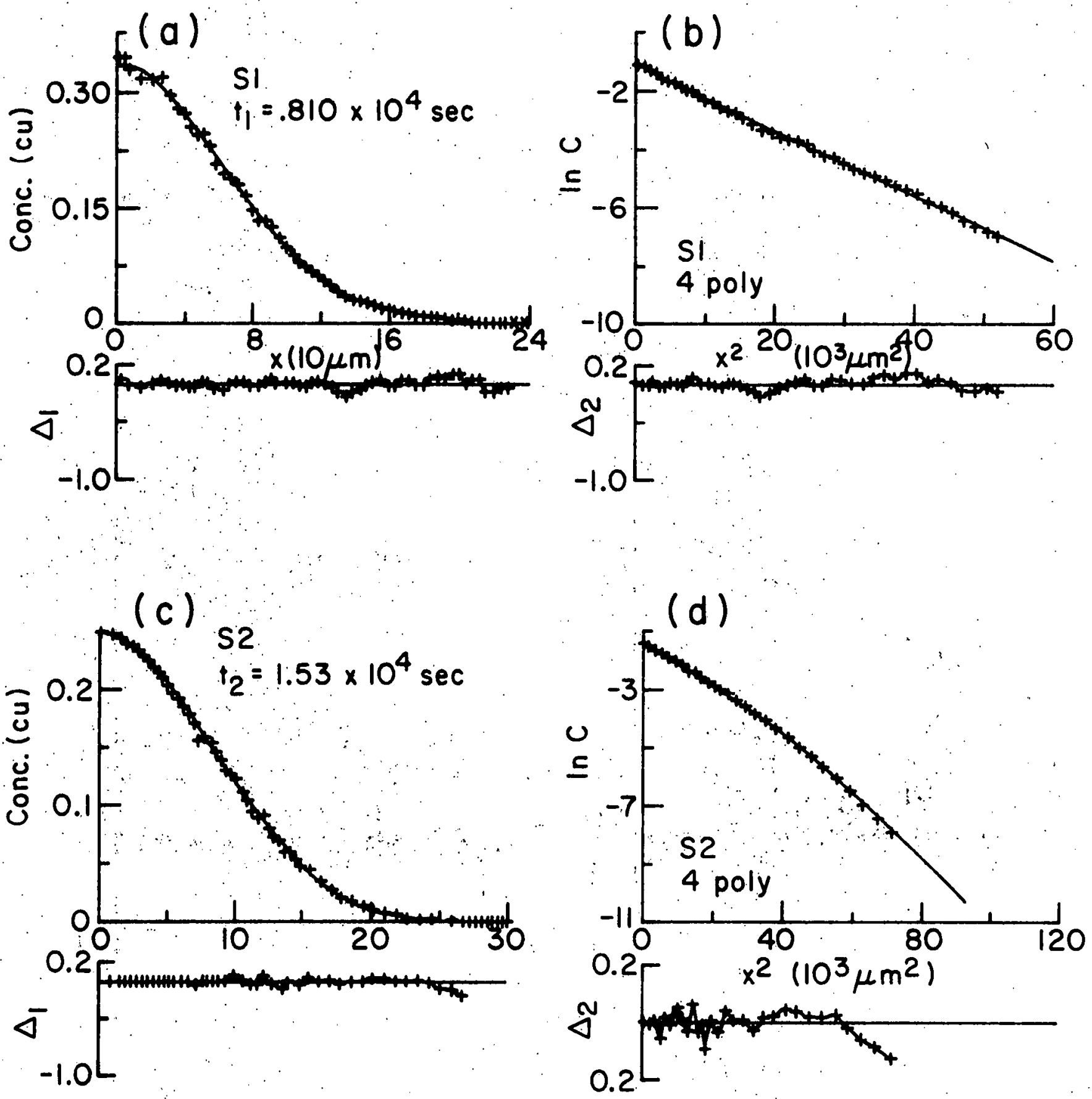

Fig. 7.4 

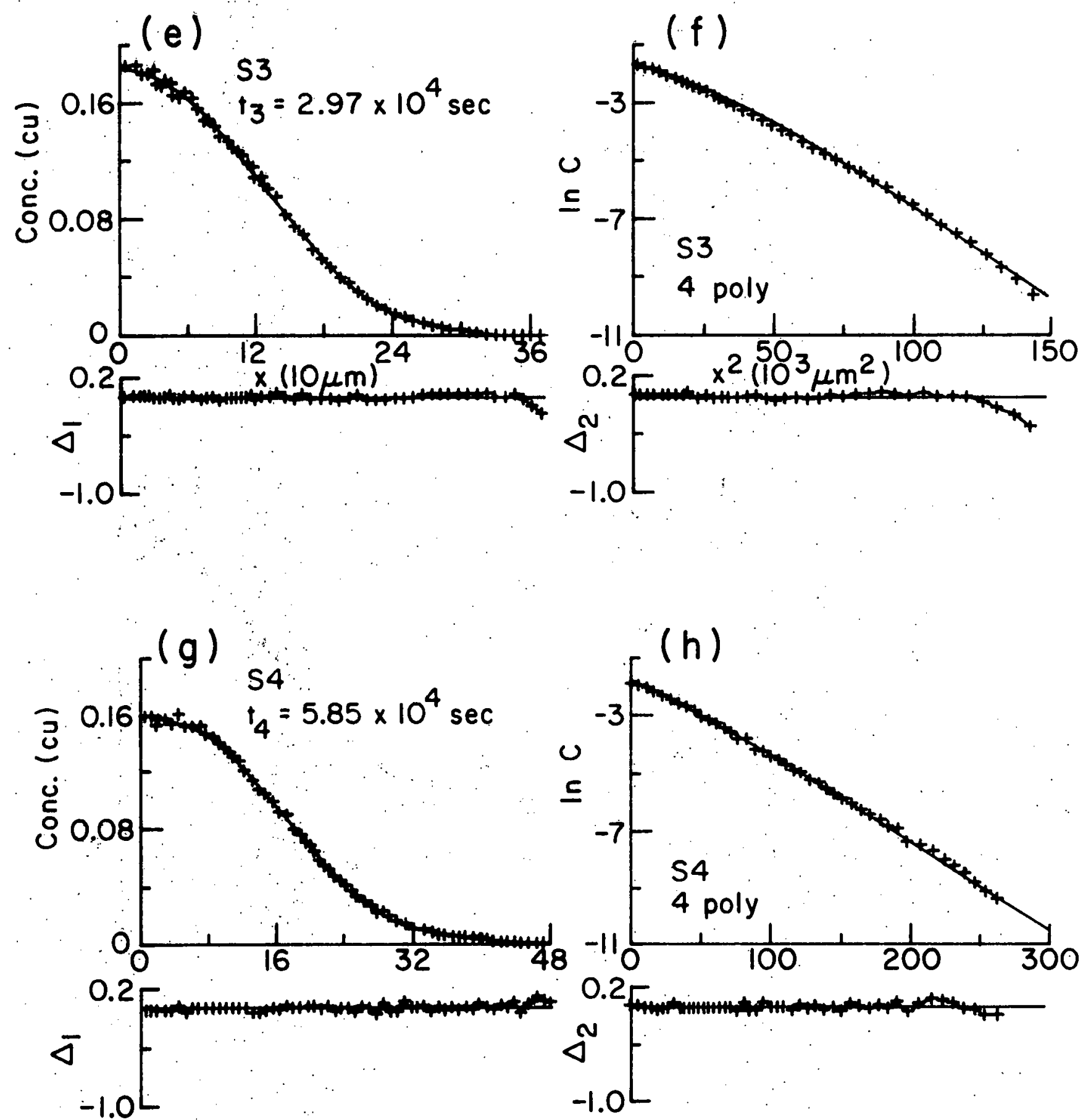

Fig. 7.4 
Figures 7.5a through 7.5h. Plots of the 'smoothed' raw data. Lowest temperature run; Average temperature: $574^{\circ} \mathrm{C}$. The presentation form follows Figure 7.1, with each individual dataset labelled as follows:

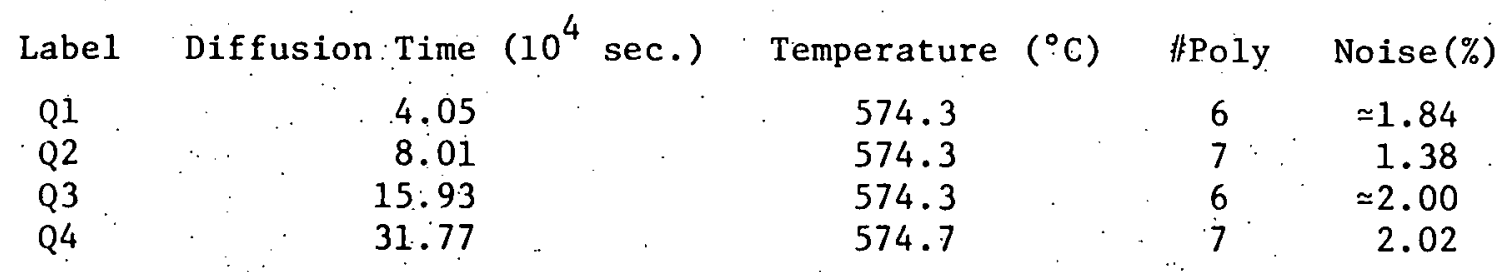


160
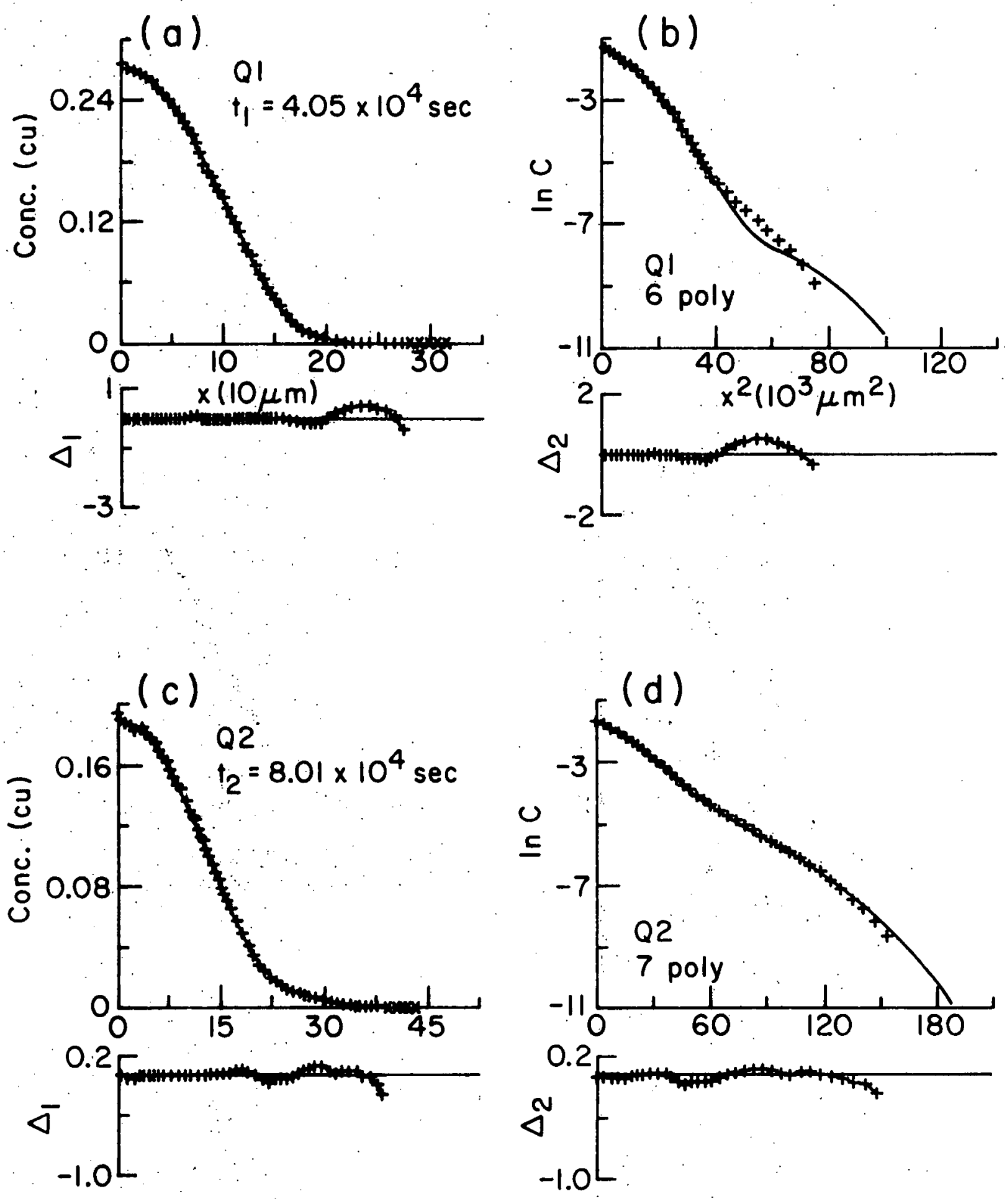

Fig. 7.5 
161
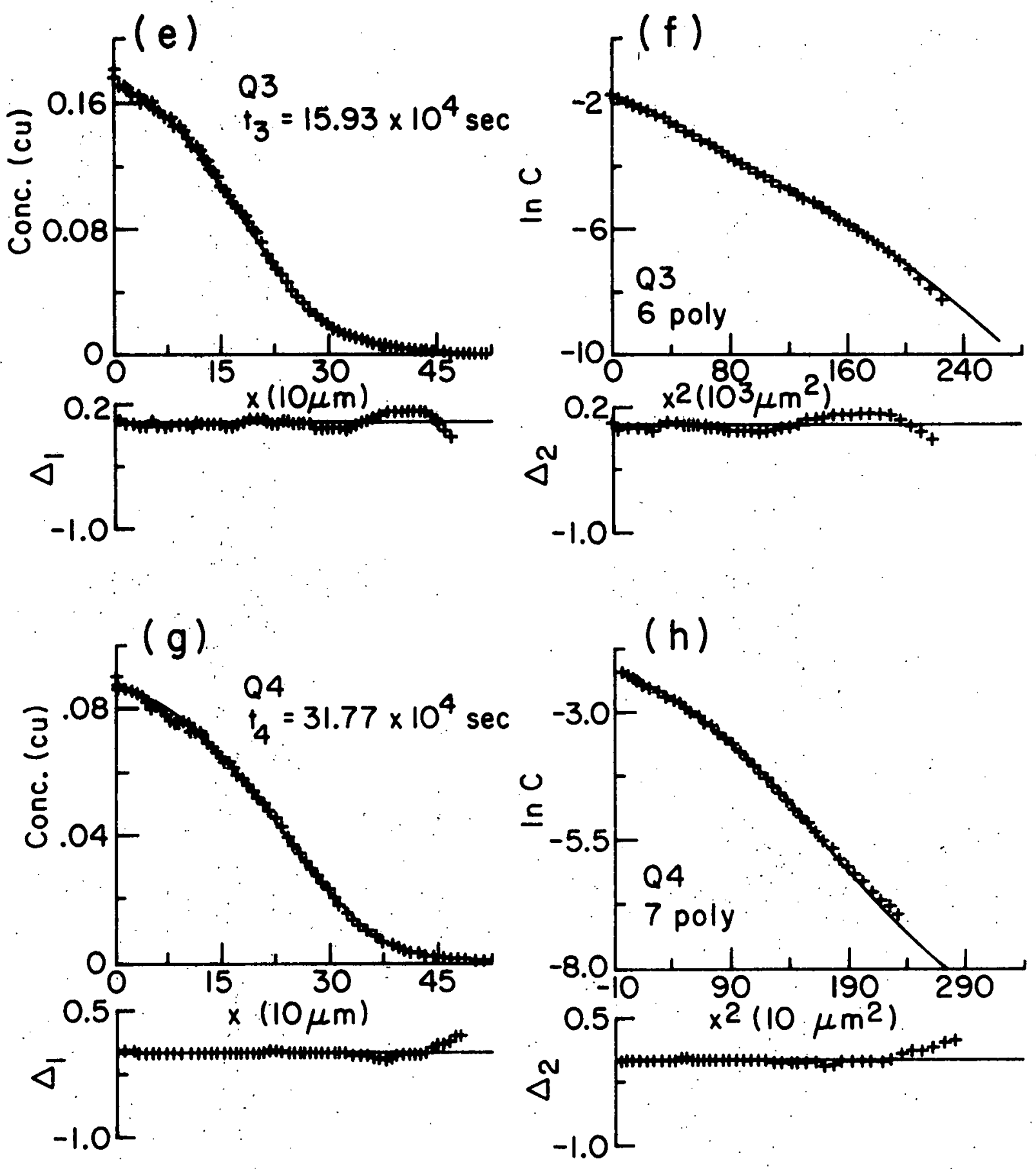

Fig. 7.5 
Figures 7.6a through 7.6d. These plot the 'smoothed' raw data for two additional profiles, both taken at the highest. temperature. The presentation follows Figure 7.1 with each individual dataset labelled as follows:

$\begin{array}{ccccc}\text { Label } & \text { Diffusion Time }\left(10^{4} \mathrm{sec}\right) & \text { Temperature }\left({ }^{\circ} \mathrm{C}\right) & \text { \#Poly } & \text { Noise (\%) } \\ \text { U3A } & 0.816 & 774.6 & 5 & 1.18 \\ \text { U4A } & 1.530 & 774.9 & 5 & 2.45\end{array}$

Since both $U 3$ and $U 4$ are data taken at the same diffusion time, temperature, and warm-up history as $U 3 A$ and $U 4 A$, respectively, the critical comparison between the datasets are in the quantity: "Total Area" $=\int \tilde{c}(x, t) d x$, which is a measure of total impurity content deposited initially on the crystal surface before diffusion. The plots on [C vs. $x$ ]-axes have been normed; $i . e ., \int c(x, t) d x=\hat{1}$, and the actual areas are as follows:

Label Total Area

U3

U3
5.637

5.630
Labe1 Tota1 Area

$\mathrm{U} 4$

U4A

5.407

2.926 

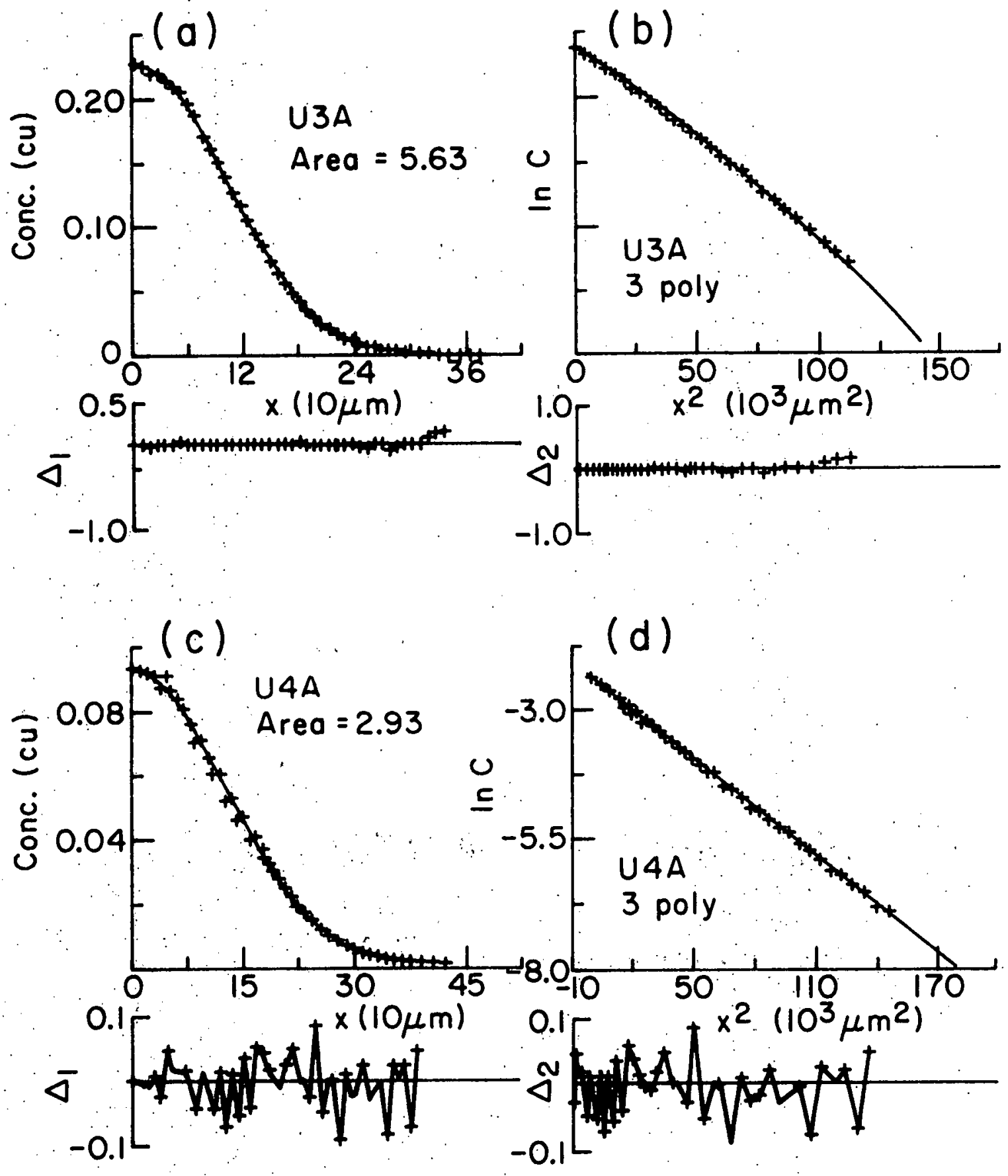

Fig. 7.6 
Figures $7.7 \mathrm{a}$ and $7.7 \mathrm{~b}$. Plots of the 5-polynomial fits of the U3-dataset compared with the U3A-dataset, presented both on [C vs: $x$ ]-axes and $\left[\ell n C\right.$ vs. $\left.x^{2}\right]$-axes. Since both datasets. were taken under identical conditions, including equivalent initial surface diffusant amounts, the difference between the two indicates the resolution of the combined system of experimental procedure and raw-data fitting procedure.

On the [C vs. $x$ ]-graph, the datasets ' $U 3 A^{\prime}$ ' match to slightly better than $1 \%(\simeq .96 \%)$. On a [ lnC vs. $x^{2}$ ]-graph, there is a slight deviation in the deep penetration region, and this is primarily due to (polynomial) $\times$ (gaussian) data fitting procedure being more inaccurate in this region. The concentration is in units: 1 cu=1 nanomole $/ \mathrm{cm}^{2}$ micron. 


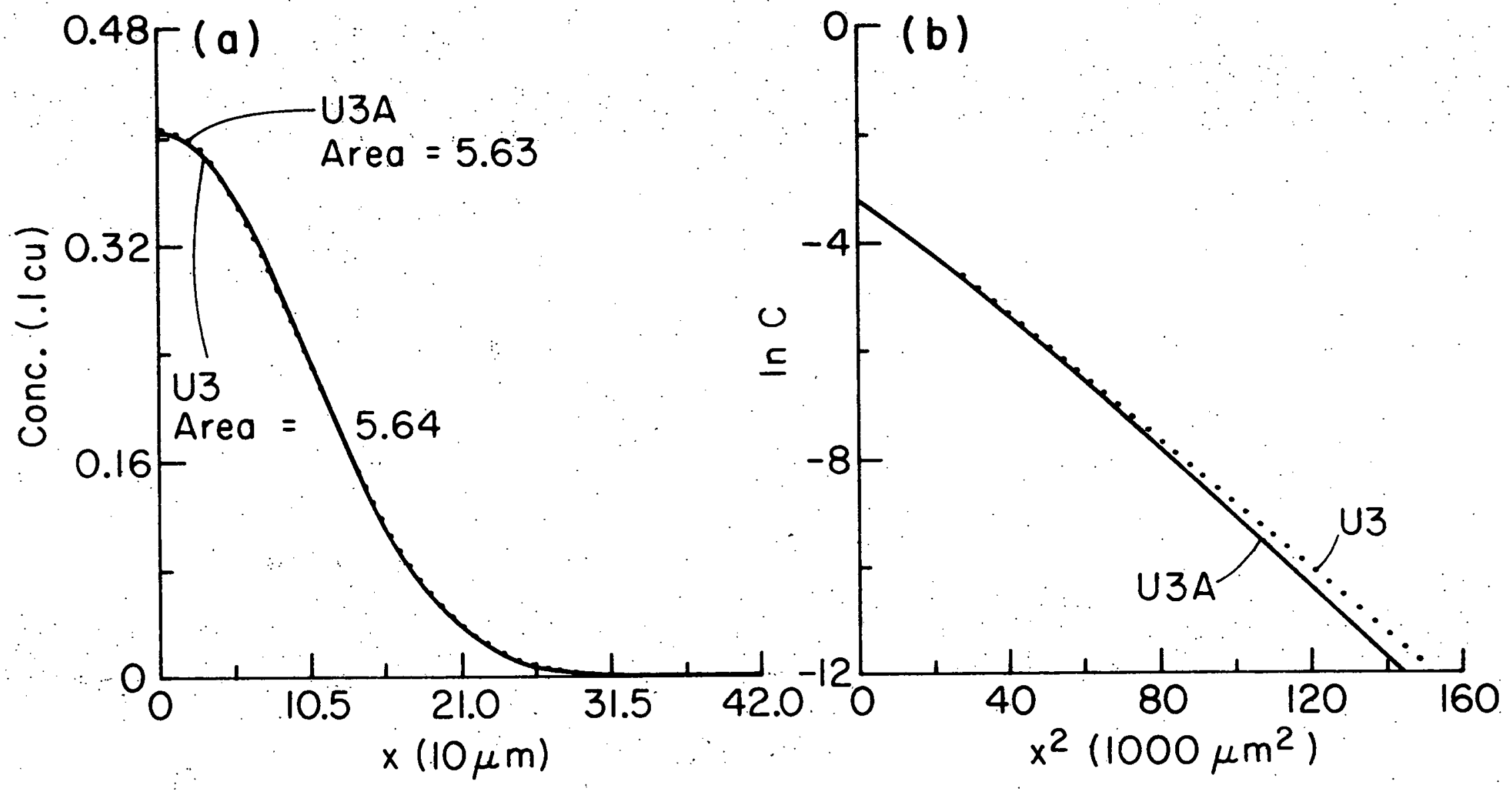

Fig. 7.7 
Figures $7.8 \mathrm{a}$ and $7.8 \mathrm{~b} . \therefore$ Plots of the 5-polynomial fits of the U4-dataset compared with the U4A-dataset. Both profiles are normed to unit area $\left(\int c(x, t) d x=\hat{1}\right)$, and they show the striking effect of changing total diffusant amount by a factor of $\approx 2$, even for this highest temperature, longest diffusion time run (where the diffusion process should be most 'normal'). The two [C vs. $x$ ]-curves in Fig. 7.8a differ by almost $12 \%$.

'U4A' is a sample with approximately half the tracer amount as other crystals in this experiment, and the profile is indeed shallower (on a [C vs. $x$ ]-plot). The [lnC vs. $\left.x^{2}\right]$-graph shows the low impurity content (U4A) profile to be nearly gaussian; the higher impurity content profile (U4) is "Curved". The high impurity content profile also has a limiting slope similar to, though slightly steeper than, the low impurity content profile. 


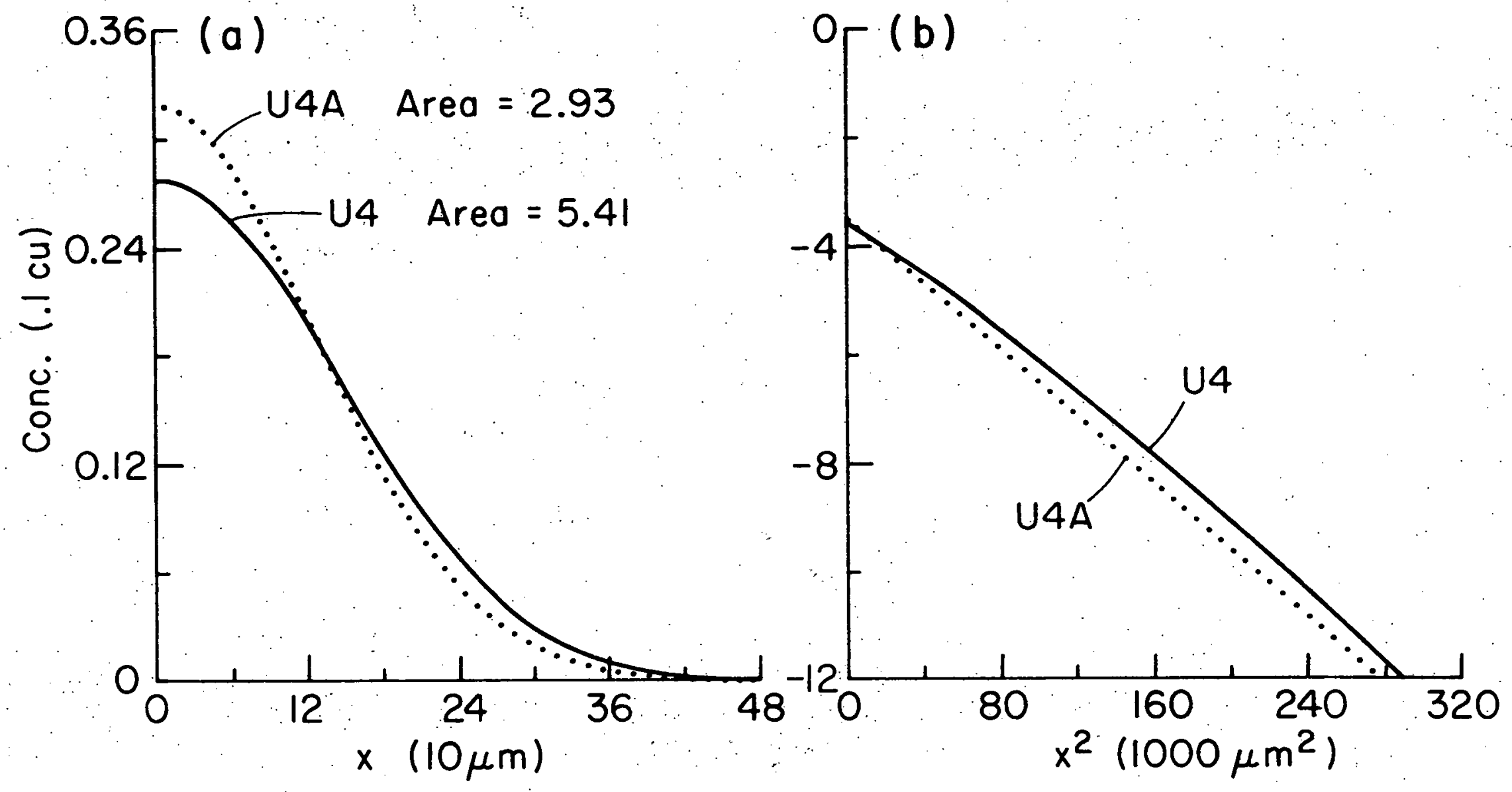

Fig. 7.8 